\title{
Oxyamine Linkers for the Protecting Group Free Synthesis of Glycoconjugates
}

By

\author{
Jaimé Caitlin Hill
}

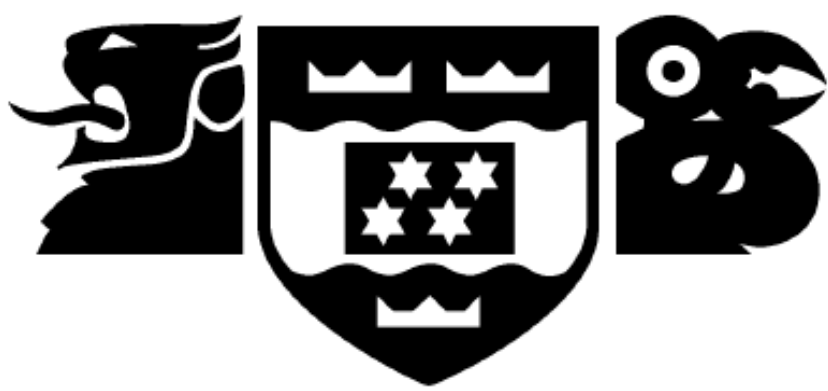

School of Chemical and Physical Sciences

Victoria University of Wellington

A thesis submitted to Victoria University of Wellington in fulfilment of the requirements for the degree of

Master of Science in Chemistry 


\begin{abstract}
Glycoconjugates, such as glycolipids and glycoproteins, are involved in a variety of cellular functions including cell-to-cell signalling and carbohydrate-protein recognition. Accordingly, glycoconjugates play important roles in health and disease and are promising new leads as carbohydrate-based therapeutics. However, for the development of glycoconjugates to study biological processes, or for the use of these adducts as therapeutics, the glycan needs to be conjugated to the carrier molecule or scaffold of choice. Many procedures for the conjugation of glycans involve lengthy protecting group strategies that install the aglycone at the start of glycan total synthesis and are therefore unsuitable for naturally derived sugars. Other glycan conjugation strategies can affect the integrity of the reducing end sugar or lead to adducts where the reducing end sugar adopts the ring-opened rather than the ring-closed form. N,O-Dialkyl oxyamine linkers, however, can be attached to the free reducing end of sugars in a single step without the need for protecting groups. This thesis therefore explores the synthesis and application of oxyamine linkers for the synthesis of glycoconjugates.
\end{abstract}

First, the synthesis of an $O$-alkyl- $N$-methyl oxyamine linker ("Type A") containing an amine at its terminus was improved by reducing the number of synthetic steps from six to four and by increasing the overall yield from $8 \%$ to $38 \%$. This oxyamine linker was then conjugated to GlcNAc in $83 \%$ yield. The hydrolytic stability of this glycosyloxyamine was then compared to that of the analogous $\mathrm{N}$-alkyl- $O$-methyl glycosyloxyamine ("Type B”). The stability of the two types of glycosyloxyamines has never been directly compared. Accordingly, it was not known whether the difference in substitution pattern between the two linkers affects their hydrolytic stability. To this end, the hydrolysis rates of the GlcNAc conjugated linkers were assessed at various $\mathrm{pH}$ values, glycoconjugate concentrations and buffer concentrations. In all instances, the "Type B" glycoside was found to have marginally better kinetic stability, while the "Type A" glycoside had marginally better thermodynamic stability, but overall, these differences were negligible. The $\mathrm{p} K_{\mathrm{a}}$ of the conjugate acid of these glycosyloxyamines was also determined to provide insight into the mechanism of hydrolysis. By considering this data, along with the observation that the rate of hydrolysis of these glycoconjugates increases with increasing buffer concentration, it was proposed that the hydrolysis of the oxyamines occurs via general acid catalysis at $\mathrm{pH}$ 4-6. 
A novel dithiol functionalised oxyamine linker was also designed and synthesised for the multivalent display of glycans on gold nanoparticles. With the successful attachment of this thiol linker to GlcNAc, the monomer unit of chitin, this work has paved the way for the future syntheses of chitin-functionalised gold nanoparticles. Such chitinfunctionalised AuNPs can be used to assess chitin's ability to invoke the asthma allergic immune response, thereby bringing the possibility of an anti-asthma vaccine a step closer to fruition. 


\section{Acknowledgements}

First and foremost, I would like to thank my supervisors, Mattie and Bridget, not only for your help, ideas and support during my Master's, but also for your belief in me, back when I was an undergrad. You took me on at the end of my second year and let me loose on your lab - I promise there were only a few broken pieces of glassware! Thank you, Mattie, for personally showing me the ropes during that time. I've come a long way since then, and have learnt so much while part of your research group.

I would also like to thank Stefan, your expertise on oxyamines made your help and guidance invaluable, especially during the hydrolysis studies. Your passion and enthusiasm for chemistry was infectious, and thank you for taking every opportunity to teach me something new. Thank you also for company during coffee breaks and doing the "Stuff" online crosswords while we waited for the HPLC to run.

Thanks also to the members of the Immunoglycomics research group. It's been great fun getting to know you and work with you guys over the past three years. Thanks especially to Jessie, my ex-fumehood buddy, for all the laughs and support. Thanks to Amy for your "amazing" dance moves - which never fail to entertain. Thanks also to Cij, Thomas and Ayesha for the coffee/Milo breaks and impromptu badminton/squash games, and Kris for your positivity and smiles in the lab. And to Emma, thanks for your support in the lab towards the end of my Master's.

To Sarah, Matt, Dylan and Cameron, thanks for suffering through undergraduate and postgraduate Chemistry with me, we had some good times! And to Susan, who went to the dark side of biology in the end, thanks for all the crazy fun times over the last 5 years.

I would like to acknowledge other academic and support staff at VUW, especially Ian for all your help with NMR. You seem to always be available and willing to help. Thanks for your positive attitude and chats about Namibia, the old "Suidwes".

Thanks to my flatmates, Dylan and Danielle - I couldn't have asked for better flatmates. Thanks for "educating" me on TV shows and movies that are a "must-see", including rewatching all 6 seasons of Game of Thrones with me. 
Thanks especially to my Mom and Dad. Without your support, I'm not sure this thesis would have been completed. This last year has been tough, for a number of reasons and your love, care and support got me through it - thank you so much. Thank you, Mom, for also taking the time to proof-read this thesis.

Finally, I would also like to acknowledge the scholarships which have funded my Master's, including the Victoria Graduate Award, the Averil Brent Scholarship in Science, two Curtis-Gordon Research Scholarships in Chemistry and the Victoria Master's by Thesis Scholarship. 


\section{List of Abbreviations and Terms Used}

\begin{tabular}{|c|c|}
\hline ABPP & Activity-based protein profiling \\
\hline Ac & Acetyl \\
\hline $\mathrm{AcOH}$ & Acetic acid \\
\hline Adjuvant & Immune activating agent added to vaccines \\
\hline Ala & Alanine \\
\hline Amylase & Enzyme which catalyses the hydrolysis of starch \\
\hline Antibody & Large protein released by immune cells to detect antigens \\
\hline Antigen & Molecule recognised by the immune system \\
\hline AuNP & Gold nanoparticle \\
\hline B cell & $\begin{array}{l}\text { Type of lymphocyte with the primary function of making } \\
\text { antibodies against antigens }\end{array}$ \\
\hline Boc & tert-Butyloxycarbonyl \\
\hline${ }^{\mathrm{BPC}} \mathrm{NeuAc}$ & $\begin{array}{l}\text { 9- } N \text {-biphenylcarbonyl-NeuAc } \alpha-(2 \rightarrow 6)-G a l \beta-(1 \rightarrow 4)- \\
\text { GlcNAc }\end{array}$ \\
\hline $\mathrm{CD} 169 / \mathrm{Sn}$ & $\begin{array}{l}\text { Sialoadhesin, a cell surface receptor specific to } \\
\text { macrophages and is a member of the siglec family }\end{array}$ \\
\hline $\mathrm{CD} 22$ & $\begin{array}{l}\text { A cell surface receptor specific to B cells and is a member } \\
\text { of the siglec family }\end{array}$ \\
\hline Chitin & A polymer of $\beta$-1,4-linked $N$-acetyl-D-glucosamine \\
\hline Chitinase & Enzyme which breaks down chitin \\
\hline COSY & Correlation spectroscopy; 2D NMR experiment \\
\hline $\mathrm{DC}$ & $\begin{array}{l}\text { Dendritic cell; immune cell that takes up and processes } \\
\text { antigens for presentation to other immune cells }\end{array}$ \\
\hline DCM & Dichloromethane \\
\hline DC-SIGN & $\begin{array}{l}\text { Dendritic cell-specific intercellular adhesion molecule-3- } \\
\text { grabbing non-integrin }\end{array}$ \\
\hline DIAD & Diisopropyl azodicarboxylate \\
\hline DMF & Dimethylformamide \\
\hline DSS & 3-(Trimethylsilyl)propane-1-sulfonic acid \\
\hline DTT & Dithiothreitol \\
\hline ECM & Extra cellular matrix \\
\hline ELISA & Enzyme linked immunosorbent assay \\
\hline Epitope & Specific part of an antigen that an antibody binds to \\
\hline EPO & Erythropoietin \\
\hline Et & Ethyl \\
\hline EtOAc & Ethyl acetate \\
\hline Eukaryotic cell & Cell which contains a membrane-bound nucleus \\
\hline FITC & Fluorescein isothiocyanate \\
\hline Flow cytometry & $\begin{array}{l}\text { Biophysical technology used to analyse multiple } \\
\text { characteristics of single cells using lasers }\end{array}$ \\
\hline Fmoc & Fluorenylmethyloxycarbonyl \\
\hline Fuc & Fucose \\
\hline GAC & General acid catalysis \\
\hline Gal & Galactose \\
\hline GBC & General base catalysis \\
\hline GFP & Green fluorescent protein \\
\hline Glc & Glucose \\
\hline GlcNAc & $N$-acetylglucosamine \\
\hline
\end{tabular}




\begin{tabular}{|c|c|}
\hline Glycosyl transferase & $\begin{array}{l}\text { Enzyme which catalyses the transfer of 'activated' } \\
\text { carbohydrates to an acceptor }\end{array}$ \\
\hline Glycosidase & Enzyme which catalyses the hydrolysis of glycosidic bonds \\
\hline Golgi apparatus & $\begin{array}{l}\text { Intracellular organelle involved in secretion and } \\
\text { intracellular transport }\end{array}$ \\
\hline GPI & Glycosylphosphatidylinositol \\
\hline $\begin{array}{l}\text { Gram-negative } \\
\text { bacteria }\end{array}$ & $\begin{array}{l}\text { Type of bacteria which have two membranes seperated by a } \\
\text { thin peptidoglycan layer }\end{array}$ \\
\hline HIV & Human immunodeficiency virus \\
\hline HMBC & $\begin{array}{l}\text { Heteronuclear multiple bond correlation; 2D NMR } \\
\text { experiment }\end{array}$ \\
\hline HPLC & High performance liquid chromatography \\
\hline HSQC & $\begin{array}{l}\text { Heteronuclear single quantum coherence; } 2 \mathrm{D} \text { NMR } \\
\text { experiment }\end{array}$ \\
\hline IL-4 & Interleukin 4 \\
\hline Integrin & $\begin{array}{l}\text { Transmembrane protein involved in anchoring cells to the } \\
\text { ECM }\end{array}$ \\
\hline$K_{\mathrm{a}}$ & Acid dissociation constant \\
\hline KIE & Kinetic isotope effect \\
\hline LacNAc & $N$-acetyl-lactosamine \\
\hline Lectin & Class of glycan-binding proteins \\
\hline $\mathrm{Le}^{\mathrm{X}}$ & Lewis $\mathrm{X}$ \\
\hline Liposome & Vesicle made from a lipid bilayer \\
\hline LPS & Lippopolysaccharide \\
\hline Lymphocyte & $\begin{array}{l}\text { Class of white blood cell part of the vertebrate immune } \\
\text { system }\end{array}$ \\
\hline Lys & Lysine \\
\hline Lysosome & $\begin{array}{l}\text { Membrane-bound compartment within the cell for the } \\
\text { catalytic breakdown of substances }\end{array}$ \\
\hline M & Molar; moles per litre \\
\hline M6P & Mannose-6-phosphate \\
\hline Man & Mannose \\
\hline MBL & Mannose binding lectin \\
\hline Me & Methyl \\
\hline $\mathrm{MHz}$ & Mega hertz \\
\hline MPL & Monophosphoryl lipid A \\
\hline MR & Mannose receptor \\
\hline $\mathrm{MsCl}$ & Methanesulfonyl chloride \\
\hline NHS & $N$-hydroxysuccinimide \\
\hline NMR & Nuclear magnetic resonance \\
\hline PAMAM & Poly(amido amine) \\
\hline PAMP & Pathogen associated molecular pattern \\
\hline PBS & Phosphate-buffered saline \\
\hline $\mathrm{PE}$ & Petroleum ether \\
\hline PEG & Polyethylene glycol \\
\hline $\mathrm{Ph}$ & Phenyl \\
\hline PRR & Pattern recognition receptor \\
\hline RDS & Rate determining step \\
\hline SAC & Specific acid catalysed \\
\hline SAM & Self assembled monolayer \\
\hline
\end{tabular}


SBC

Siglec

Sn/CD169

SPPS

SPR

ST

Streptavidin

${ }^{\mathrm{TCC}} \mathrm{Neu} 5 \mathrm{Ac}$

$\mathrm{T}$ cell

TEA

TFA

Th1

Th17

Th2

THF

TLC

TLR

$t_{1 / 2}$

UV/VIS

VUW
Specific base catalysis

Sialic-acid-binding Ig-like lectin

Sialoadhesin, a cell surface receptor specific to macrophages and is a member of the siglec family Solid phase peptide synthesis

Surface plasmon resonance

$(2,6)$-sialyl T antigen

Protein with a high affinity for biotin

9- $N$-(4H-thieno[3,2-c]chromene-2-carbamoyl)-Neu5Aca$(2 \rightarrow 3)$-Gal $\beta-(1 \rightarrow 4)$-GlcNAc

Type of lymphocyte involved in the recognition and destruction of antigens

Triethylamine

Trifluoroacetic acid

T-helper 1

T-helper 17

T-helper 2

Tetrahydrofuran

Thin layer chromatography

Toll-like receptor

Half-life

Ultra violet/visual

Victoria University of Wellington 


\section{Table of Contents}

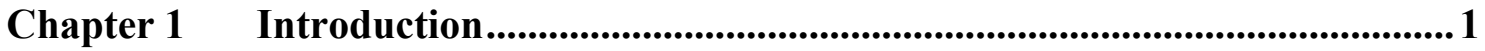

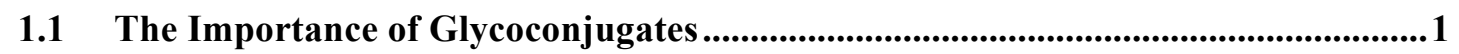

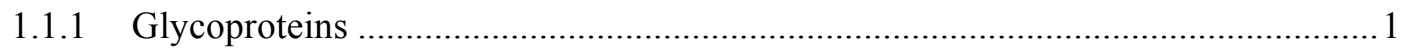

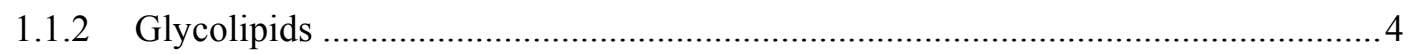

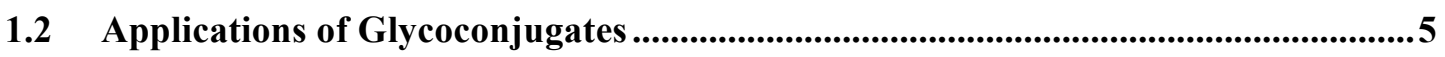

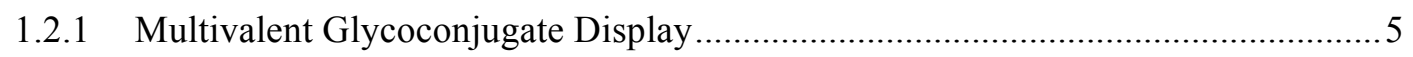

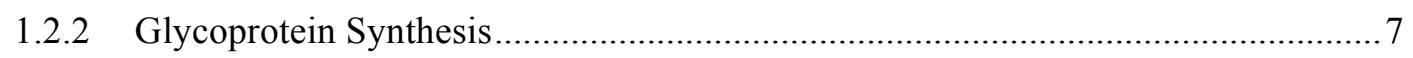

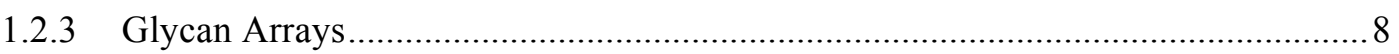

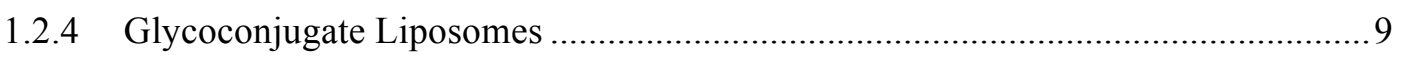

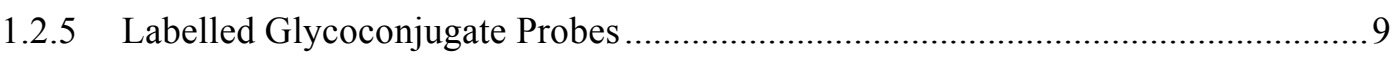

1.3 Conjugation Strategies ...................................................................................................11

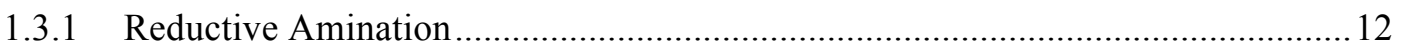

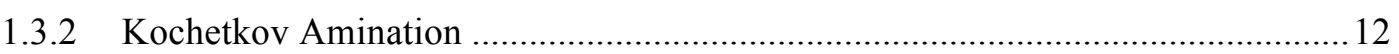

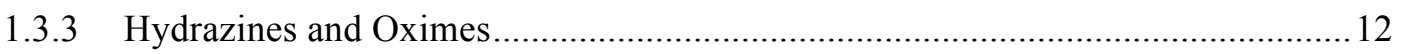

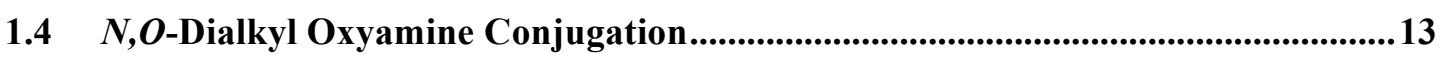

1.5 Scope and Limitations of Oxyamine Glycan Conjugation .....................................16

$1.6 \quad \mathrm{~N}, \mathrm{O}$-Alkyl- $\mathrm{N}$-Glycosyl Oxyamines in Synthesis ...................................................17

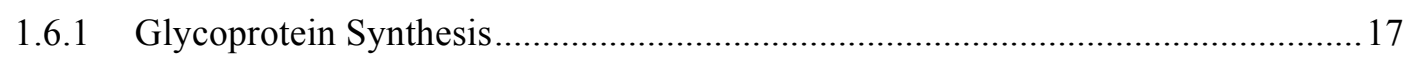

1.6.2 Oligosaccharide Mimetics .................................................................................... 18

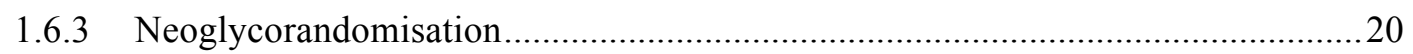

1.7 Oxyamine Glycoside Stability ......................................................................21

1.8 The Application of $N, O$-Dialkyl Oxyamine Linkers for the Multivalent Display of

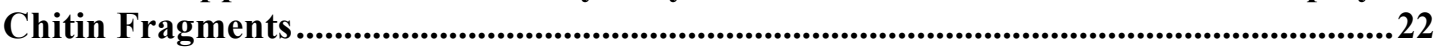

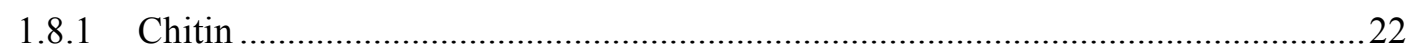

1.8.2 Gold Nanoparticles for the Multivalent Display of Antigens ................................23

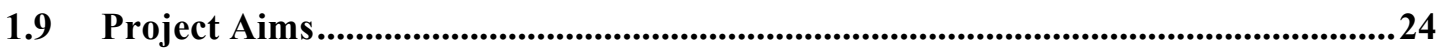

Chapter 2 Synthesis and Hydrolytic Stability of $N$ - and $O$-Methyloxyamine Linked Glycoconjugates ..........................................................................................................25

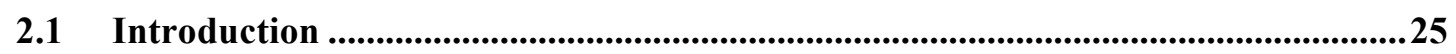

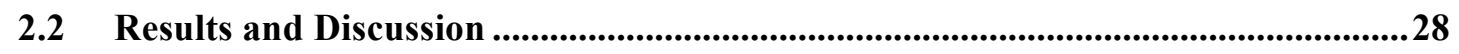

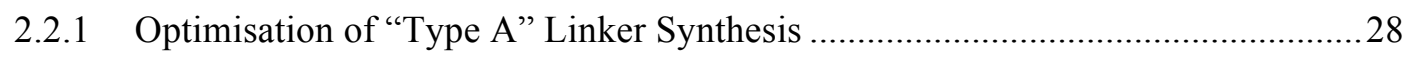

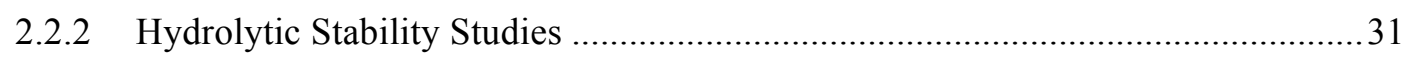

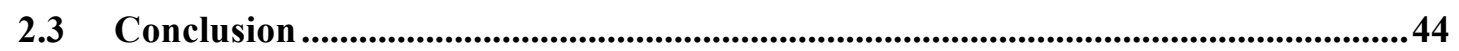

Chapter $3 \quad \mathrm{p} K_{\mathrm{a}}$ Determination of the Conjugate Acid of Glycosyloxyamines .... 45

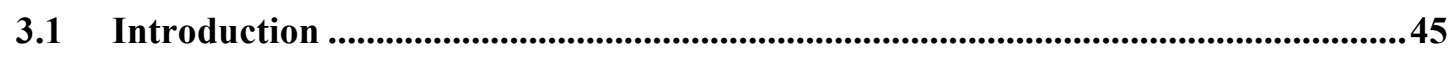

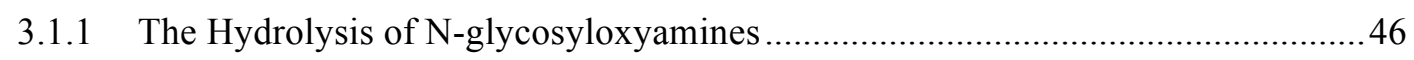

3.1.2 Acid and Base Catalysis ..................................................................................... 48

3.1.3 Methods for the Determination of $\mathrm{pK}_{\mathrm{a}}$ Values .......................................................5 50

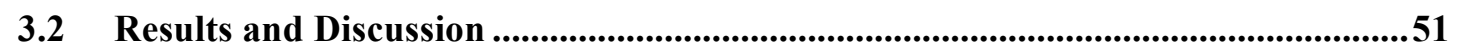

3.2.1 Model Glycoconjugate Synthesis ......................................................................... 51

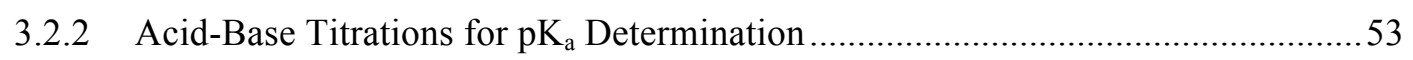

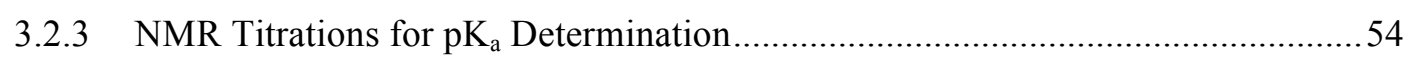


3.2.4 The Mechanism of Hydrolysis of Glycosyloxyamines ........................................59

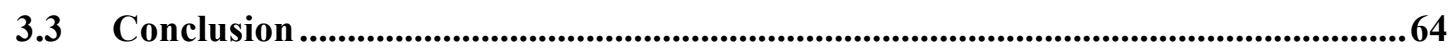

\section{Chapter 4 Synthesis and Application of Thiol Functionalised}

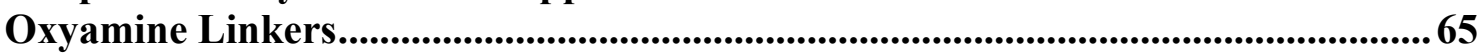

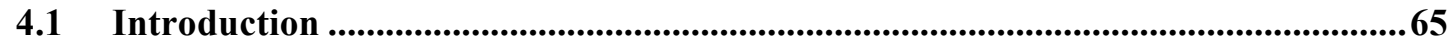

4.1.1 Gold Nanoparticles as a Multivalent Display Scaffold ………….........................65

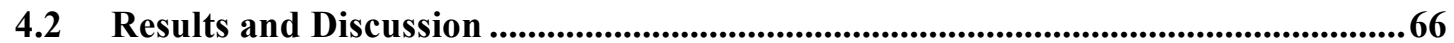

4.2.1 Sulfur Functionalised Linker Design and Synthesis ...........................................66

4.2.2 Glycosylation Reactions using the Trithiol Functionalised Oxyamine Linker .......80

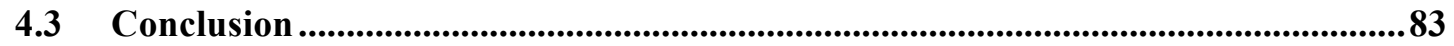

Chapter 5 Conclusion and Future Prospects........................................................85

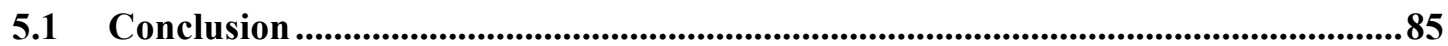

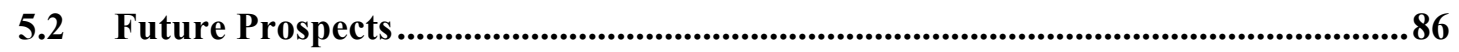

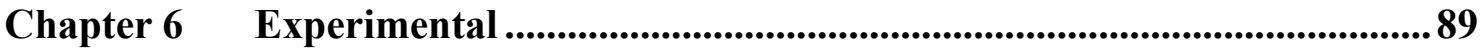

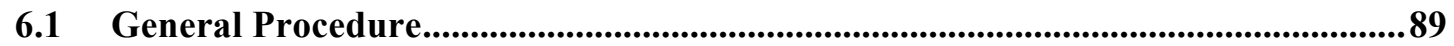

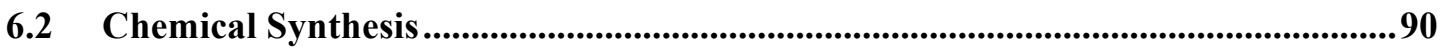

6.3 Hydrolytic Stability Experiment ..................................................................................98

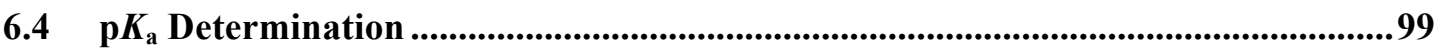

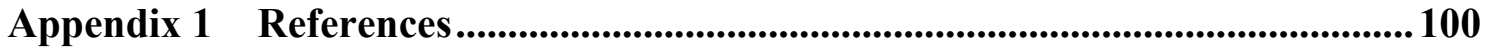

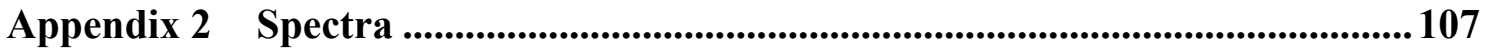




\section{Chapter 1}

\section{Introduction}

\subsection{The Importance of Glycoconjugates}

Glycoconjugates are a class of carbohydrate-containing molecules in which the carbohydrate structure, or glycan, is covalently bound to a non-carbohydrate species, such as a lipid or a protein to form glycoconjugates such as glycolipids and glycoproteins. Glycoconjugates are an essential part of the glycocalyx, a carbohydrate rich layer which coats all cells. The glycocalyx is important for cell-to-cell signalling events such as host cell recognition and communication, as well as cellular differentiation, adhesion and locomotion. ${ }^{1}$ The covalent addition of glycans to proteins occurs as a post-translational modification and is the most abundant post-translational modification sustained by proteins, with more than $50 \%$ of all proteins being glycoproteins. ${ }^{2,3}$ Moreover, there is great diversity in the glycan structure, which is attributed to the variety of ways in which the individual monosaccharide building blocks can be assembled. ${ }^{4}$ The attachment of glycans to proteins also alters the overall tertiary structure of the protein. Thus, the proper function of glycoproteins, as well as protein folding, transportation and location, is highly dependent on their glycosylation pattern. Improper glycosylation of these proteins often results in many human diseases, including cancers. ${ }^{5}$

\subsubsection{Glycoproteins}

Glycoproteins with the same protein backbone, but which differ in the number and/or type of glycans attached are known as glycoforms. These different glycosylation patterns can affect how the protein is recognised or identified within or on the surface of cells. A pertinent example is the $\mathrm{ABO}$ blood group classification, whereby the blood groups (A, $\mathrm{B}, \mathrm{AB}$ and $\mathrm{O}$ ) are determined by different glycoforms of the same protein expressed on the cell surface of red blood cells (Figure 1). People with the A glycan antigen expressed on their red blood cells have B antigen antibodies in their blood, and vice versa. As a result, a blood transfusion from a B blood group donor will be unsuccessful in an A blood group host as the immune system of the recipient will recognise this B antigen as 
"foreign" and try to eliminate it, which in turn can lead to patient death. ${ }^{6}$ Indeed, understanding the importance of different blood groups has led to modern life-saving blood transfusion methods.
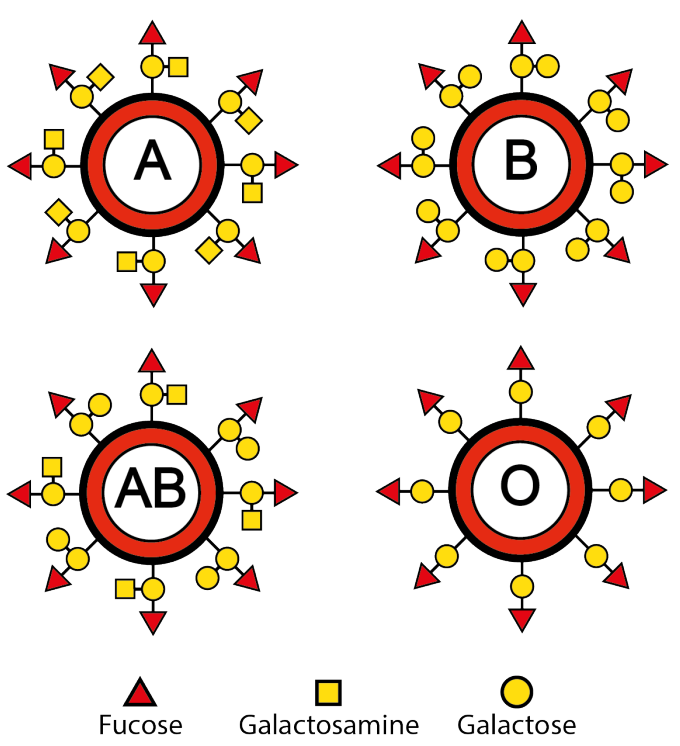

Figure 1. ABO blood group glycans, " $A$ ", " $B$ ", " $A B$ " and " $O$ ”.

Proteoglycans (glycoproteins with excessive levels of glycosylation) also have important structural roles. For example, they form one of the main components of the extracellular matrix (ECM), which is the area directly surrounding cells in tissues and which occupies a substantial portion of the overall volume of the tissue (Figure 2). The ECM has enough rigidity to hold the cells of the tissue together and is resistant to compressive forces while harbouring high levels of viscosity to allow for the migration of other cells, such as fibroblasts, through the tissue. It is the unique composition of fibrous proteins such as collagen and proteoglycans which gives the ECM these properties. ${ }^{7}$ Cells also use proteoglycans, such as fibronectin, to anchor themselves to other components of the ECM or to other cells. ${ }^{8}$ 


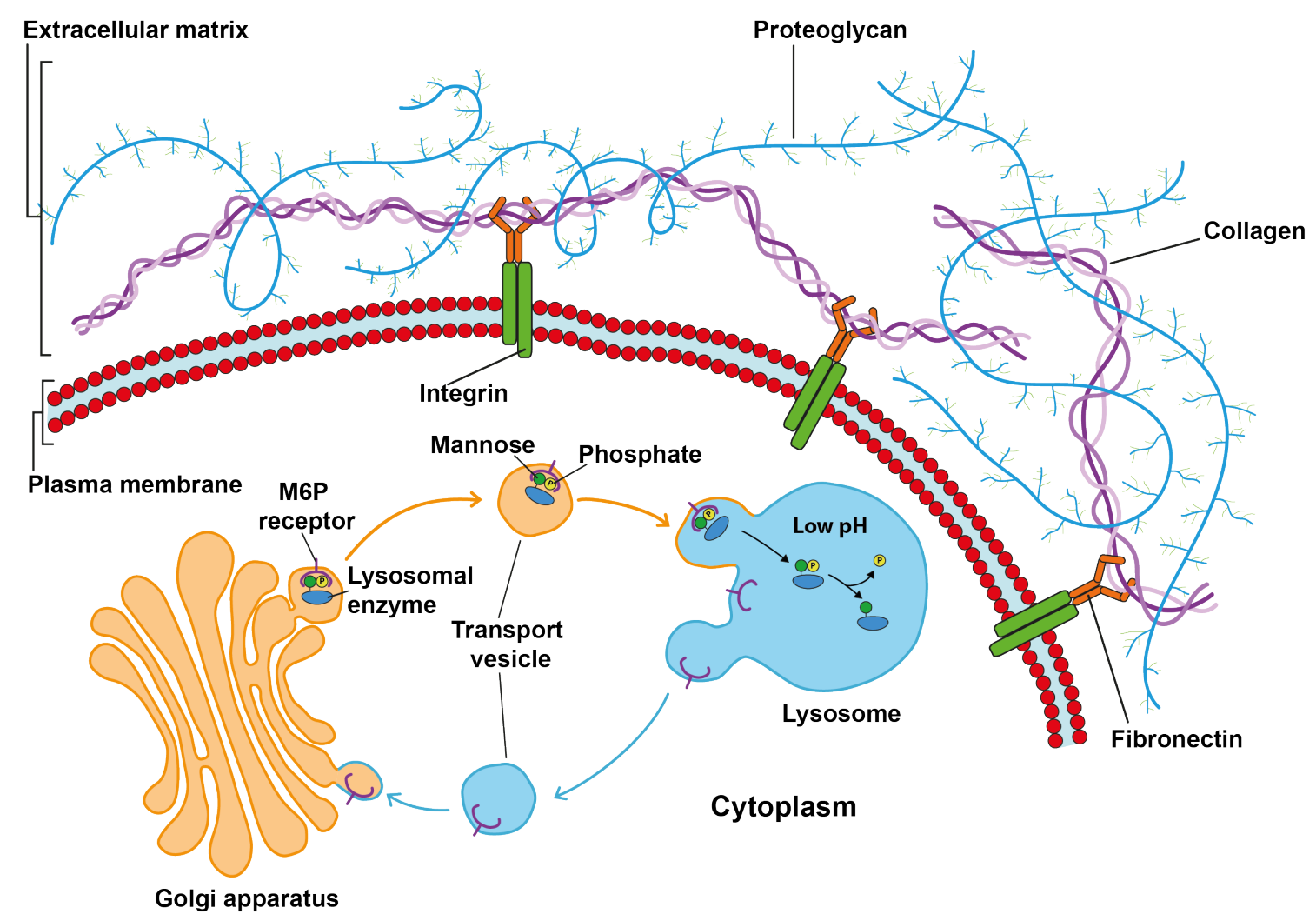

Figure 2. Cells contain an extracellular matrix mainly consisting of proteoglycans and collagen. Inside cells, further proteins are glycosylated, including lysosomal proteins which receive a mannose-6-phosphate tag so that once they leave the golgi apparatus, they are correctly delivered to lysosomes. The mannose-6-phosphate receptor then returns to the golgi to retrieve more lysosomal proteins.

The temporary glycosylation of some newly made proteins is also crucial to target them to specific organelles within the cell. For example, enzymes required in the lysosomes need to receive an oligosaccharide tag with a terminal mannose-6-phosphate residue while in the golgi apparatus in order to be transported to the lysosomes. ${ }^{9}$ Inclusion cell disease (mucolipidosis II) results when there is a deficiency in one of the transferase enzymes necessary to attach the mannose-6-phosphate tag to the lysosomal proteins. Consequently, these enzymes are incorrectly transported to the exterior of the cells where they interfere with the extracellular matrix and surrounding connective tissue. In addition, a build-up of debris in the lysosomes of cells occurs, as the debris cannot be degraded (Figure 2). Accordingly, patients with mucolipidosis II fail to grow properly in their first years of life and usually die before the age of seven. ${ }^{10}$ 


\subsubsection{Glycolipids}

Glycolipids also form an important class of biological glycoconjugates. They are often found as a component of the cell wall in eukaryotic and bacterial cells. The hydrophobic lipid tails slot into the lipid bilayer of the cellular membrane while the glycan moieties are exposed on the surface of the cell. ${ }^{7}$ This makes glycolipids important in processes such as cell adhesion and cell recognition. The cell surfaces of many bacteria are highly decorated with glycolipids and glycan displays not found on eukaryotic cells. As a result, the immune system has become quite efficient at recognising these foreign cells through the use of pattern recognition receptors (PRRs). ${ }^{11}$ Common PRRs include mannose binding lectin (MBL), toll-like receptors (TLRs) and DC-SIGN (dendritic cell-specific intercellular adhesion molecule-3-grabbing non-integrin). ${ }^{12}$ For example, lipopolysaccharide (LPS) is a glycolipid present on the cell surface of gram-negative bacteria, and is recognised by TLR-4 on innate immune cells, which in turn results in the activation of the immune system (Figure 3). The ability of the immune system to recognise glycolipids can also be exploited in the development of more effective vaccines. Accordingly, monophosphoryl lipid A (MPL), a less toxic version of LPS, is commonly used as a vaccine adjuvant. The adjuvant activates the immune system, thereby enhancing the efficiency of the vaccine antigen. An antigen is a specific epitope from the pathogen against which the vaccine is targeted. ${ }^{13}$

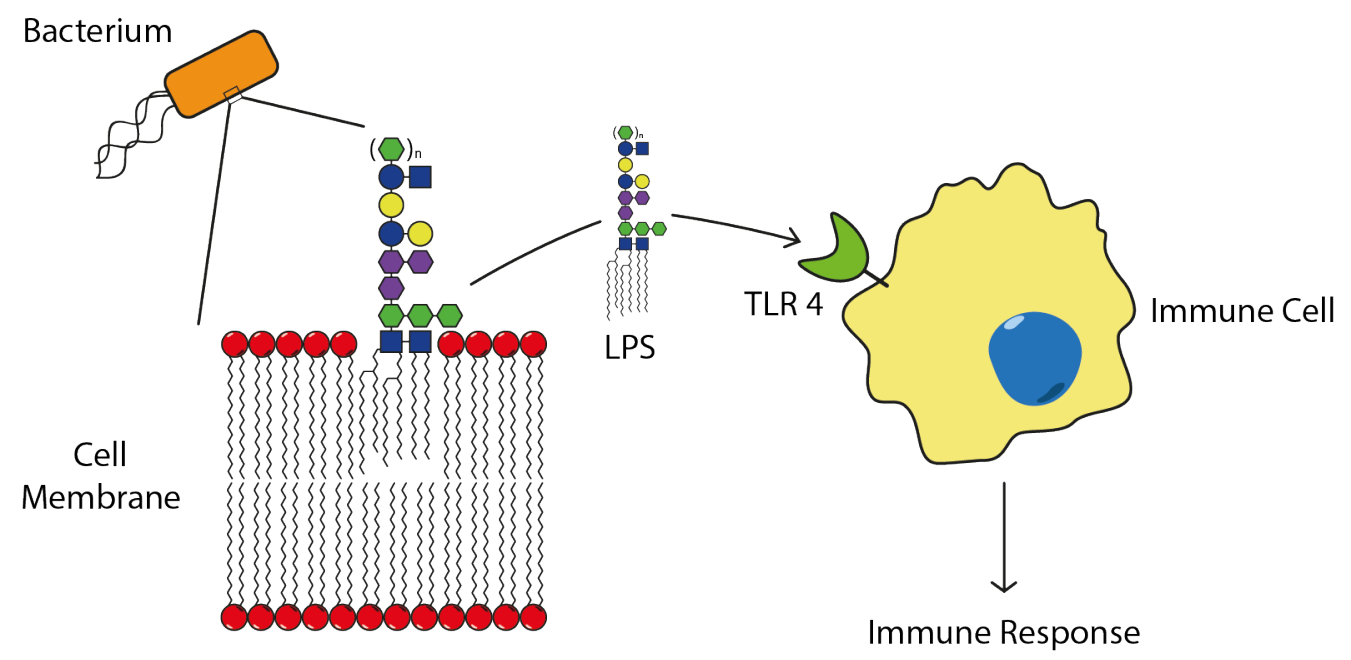

Figure 3. Lipopolysaccharide (LPS) present on the surface of Gram-negative bacteria bind toll-like receptor 4 (TLR-4) on innate immune cells thereby initiating an immune response. 


\subsection{Applications of Glycoconjugates}

The vast array of biological functions attributed to glycoconjugates has sparked much interest in the development of strategies to allow for the synthesis of glycoconjugates. Naturally derived glycoconjugates are often difficult to isolate and purify, and therefore the preparation of their synthetic analogues/mimetics is vital to the exploration of the full potential of the glycoconjugates. These synthetic strategies ${ }^{14,15}$ are adapted for the synthesis of glycoproteins, ${ }^{16}$ glycan arrays, ${ }^{17,}{ }^{18}$ glycan display on multivalent scaffolds, ${ }^{19}$ glycoconjugate liposomes ${ }^{20}$ and labelled glycoconjugate probes, ${ }^{21}$ for both therapeutic and research applications (Figure 4). Moreover, isolated or synthetically derived carbohydrates used in glycoconjugate synthesis often need to be functionalised with a linker or spacer group in order to be attached to the substrate of choice.

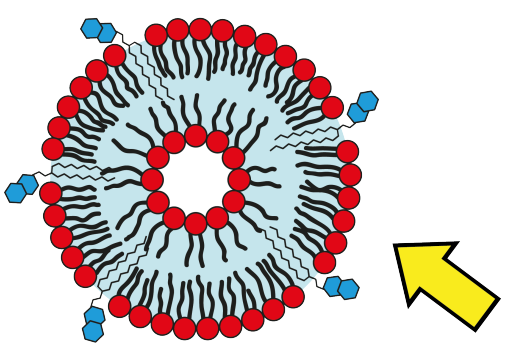

Glycoconjugate Liposomes

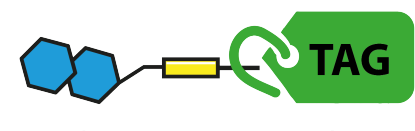

Glycoconjugate Probes

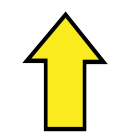

\section{Glycoconjugates}
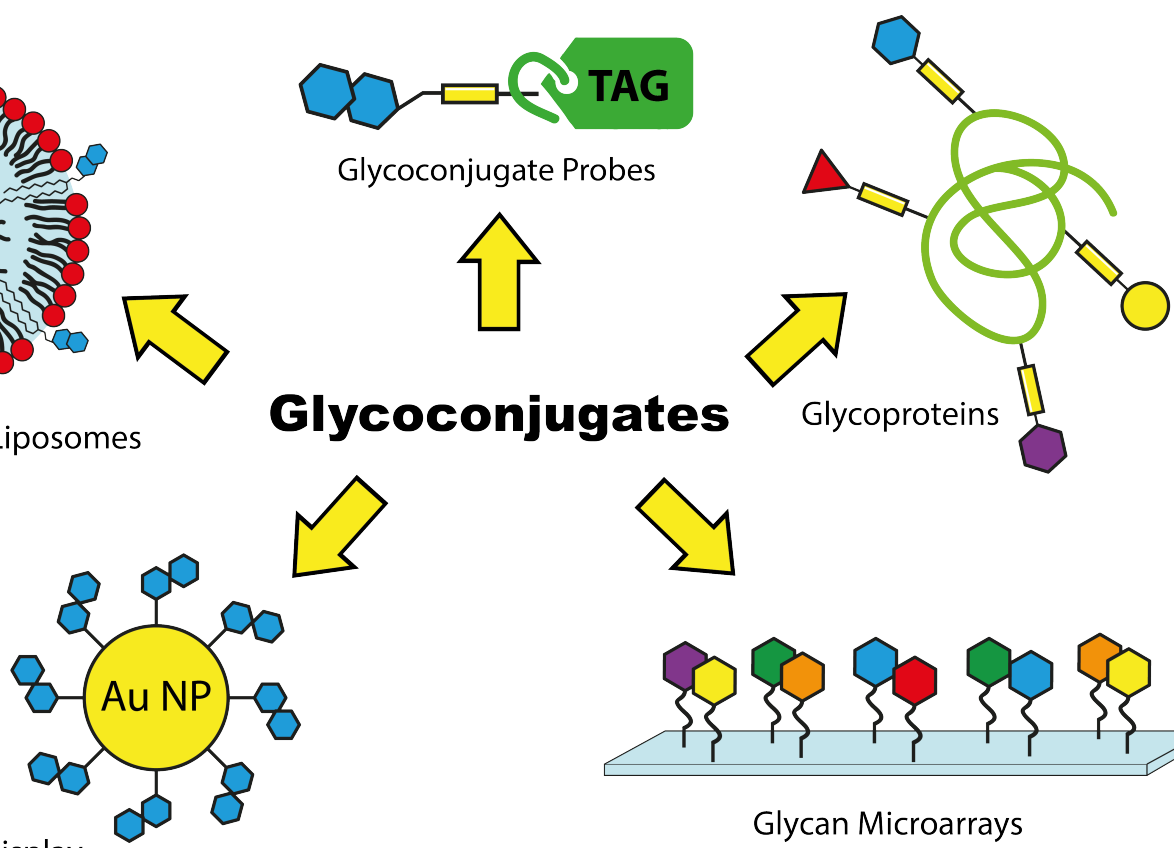

Multivalent Display

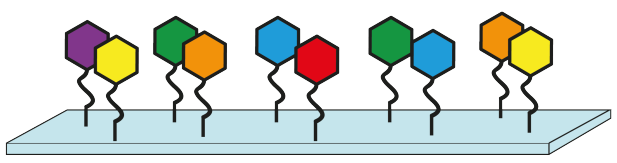

Glycan Microarrays

Figure 4. Summary of the applications of glycoconjugates. These include multivalent displays (such as gold nanoparticles), liposomes containing glycolipids, glycoconjugate probes which contain a tag (such as a fluorescent group for flow cytometry), glycoproteins and glycan microarrays.

\subsubsection{Multivalent Glycoconjugate Display}

The diversity and sheer number of glycans that exist in and on all living cells has ensured the existence of many different glycan-binding proteins, including lectins. Accordingly, 
these proteins can serve as drug targets for carbohydrate-based therapeutics to invoke cellular responses.

Most small molecule carbohydrate-based drugs act as inhibitors for glycosyl transferases and glycosidases. Acarbose, for example, inhibits glucosidases and amylases within the gut to control blood glucose levels for the treatment of type II diabetes mellitus. ${ }^{19}$ Other small molecule sugar analogues have been shown to be very effective enzyme inhibitors by mimicking the transition state of their target's natural substrate. ${ }^{22}$ Unfortunately, small molecule glycoconjugates are inefficient for the targeting of glycan binding proteins such as lectins. This is because lectins have a low affinity for their glycan substrates and rely on multivalent binding (high avidity) in order to be activated. In this regard, carbohydrates can be linked to multivalent scaffolds such as proteins, dendrimers or nanoparticles to enhance lectin avidity. As an example, the Van Kooyk research group used glycodendrimers to target the delivery of vaccine antigens to dendritic cells (DCs). ${ }^{23}$ DCs are key to activating the immune system as they present antigens to T-cells, which in turn induces a potent immune response. Van Kooyk and co-workers manufactured poly(amido amine) (PAMAM) dendrimers which were conjugated through the use of a linker to glycopeptides consisting of the glycan Lewis ${ }^{\mathrm{b}}$, a known DC-SIGN ligand, and the peptide antigen of choice (Figure 5). The resulting glycodendrimer generated stronger DC stimulation and thus T-cell activation than single conjugates of the Lewis glycan to the peptide antigen alone. ${ }^{23}$ In the following year, the same research group was involved in manufacturing gold nanoparticles for the multivalent display of a DC-SIGN binding glycan through the use of a bi-functional linker. ${ }^{24}$ 


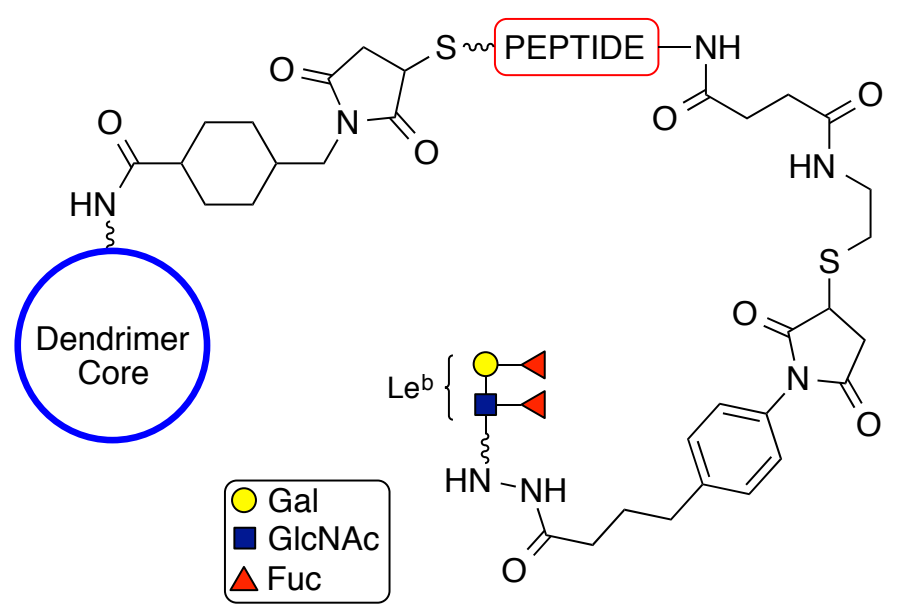

Figure 5. Van Kooyk's glycopeptide dendrimer: a PAMAM dendrimer core functionalised with primary amines is linked to a peptide through a maleimide linker. Reductive amination links the $N$-terminus of the peptide to Lewis ${ }^{b}$ via a hydrazide linker. ${ }^{23}$

\subsubsection{Glycoprotein Synthesis}

Synthesised glycoproteins and glycoprotein mimetics have found a variety of applications. Glycans are often conjugated to proteins via a linker as a means to achieve multivalent glycan display to enhance binding interactions towards glycan binding proteins such as lectins. Proteins can also function as carriers for carbohydrates in vaccines, however when carbohydrates are used as the antigen in a vaccine, they need to be conjugated to an immunogenic protein in order to generate antibodies with a high affinity for their carbohydrate targets. A successful carbohydrate-based vaccine is the Cuban Hib vaccine. This vaccine consists of a pentasaccharide structure from a capsular polysaccharide antigen belonging to Haemophilus influenzae type b conjugated to the tetanus toxoid protein. ${ }^{25}$

Certain key glycans are often up-regulated or only expressed on the surface of many types of cancerous cells, thereby acting as tumour biomarkers and/or as promising target antigens for anti-cancer vaccines. Examples include the truncated $O$-glycosylated mucins, which are glycoproteins commonly expressed on the surface of many different types of cancers. ${ }^{26}$ In 1997, Danishefsky and co-workers synthesised a tumour-associated mucin motif for the development of anti-cancer vaccines. The authors synthesised $(2,6)$-sialyl $\mathrm{T}$ antigen (ST antigen) (a glycan commonly expressed on mucins and glycoproteins associated with many different cancers) and attached it to serine and threonine building blocks that were used in solid phase peptide synthesis (SPPS). ${ }^{27}$ Similarly, Bertozzi and 
co-workers incorporated an aminooxy moiety onto another tumour-associated antigen (the Tn antigen) in order to ligate it to ketone functionalised amino acids present on presynthesised peptide chains. The generated oxime-linked mucin mimics from this strategy also have great potential for use in cancer vaccines. ${ }^{28}$

The isolation and purification of naturally sourced glycoproteins in a single glycoform is often very difficult. As a result, glycoprotein synthesis also has applications in recreating naturally occurring glycoproteins for fundamental studies or for use in protein replacement therapy. An elegant example is the work by Danishefsky and co-workers, who recently synthesised a single glycoform of erythropoietin (EPO) using convergent aspartylation to conjugate the glycans to the peptide backbone. ${ }^{29}$

\subsubsection{Glycan Arrays}

Carbohydrate microarrays offer the ability to perform high-throughput analysis of glycanprotein interactions. The chip-based format requires glycan-linker conjugates to be immobilised onto a surface. Carbohydrate-protein binding is then analysed through fluorescence, mass spectrometry, or even surface plasmon resonance (SPR) if the glycans are immobilised onto an SPR surface. ${ }^{30}$ Many different glycans can be screened on the same chip, which allows for the fast and efficient discovery of new carbohydrate ligands for carbohydrate binding proteins. Glycan microarrays can also be used to detect specific carbohydrate binding antibodies. This is useful in the diagnosis of bacterial and viral diseases as well as cancers. Microarrays are also used as validation methods for vaccines in order to detect if a suitable and desirable antibody response has been elicited in a vaccinated individual. ${ }^{17}$ In order to screen for glycan ligands, carbohydrate libraries are often used, which in turn require a facile and effective method to transform the carbohydrates into the glycoconjugate counterparts needed to immobilise them onto the chip surface. Many conjugation strategies, however, do not allow for the use of naturally derived carbohydrates due to the need for lengthy protecting group manipulations which are not feasible on the small scale. ${ }^{31}$ Consequently, linking methodologies which allow for the conjugation of all carbohydrates with a free reducing end are typically used during the construction of glycan arrays. 


\subsubsection{Glycoconjugate Liposomes}

Ligand-targeted liposomes provide a means of selectively delivering therapeutic agents, such as drugs, to specific cell types. This increases the efficacy of the drugs as it can increase drug stability and reduce the side-effects of the drug. Paulson and co-workers utilised glycoconjugate liposomes for the targeted delivery of doxorubicin (an anticancer drug) to B-cells for the treatment of B-cell lymphoma. ${ }^{32}$ The expression of the cell surface receptor CD22, a member of the sialic-acid-binding, Ig-like lectin (siglec) family, is specific to B-cells. 9- $N$-Biphenylcarbonyl-NeuAc $\alpha-(2 \rightarrow 6)-G a l \beta-(1 \rightarrow 4)-G l c N A c$ $\left({ }^{\mathrm{BPC}} \mathrm{NeuAc}\right)$, a glycan which targets CD22, was conjugated to a PEGylated lipid through an ethylamine linker (Figure 6). Liposomal nanoparticles loaded with doxorubicin were then constructed to display the ${ }^{\mathrm{BPC}}$ NeuAc lipid conjugate and were shown to be selectivly endocytosed by B-cells to extend life in a xenograft model of human B-cell lymphoma. In further work, Paulson and co-workers also developed liposomal nanoparticles which expressed 9- $N$-(4H-thieno[3,2-c]chromene-2-carbamoyl)-Neu5Ac $\alpha-(2 \rightarrow 3)$-Gal $\beta-(1 \rightarrow 4)$ GlcNAc $\left({ }^{\mathrm{TCC}} \mathrm{Neu} 5 \mathrm{Ac}\right)$ lipid conjugates. ${ }^{\mathrm{TCC}} \mathrm{Neu} 5 \mathrm{Ac}$ specifically targets sialoadhesin ( $\mathrm{Sn}$, CD169), another member of the siglec family specific to macrophages. In this way, vaccine antigens, for example, can be selectivly delivered to macrophages. ${ }^{33}$

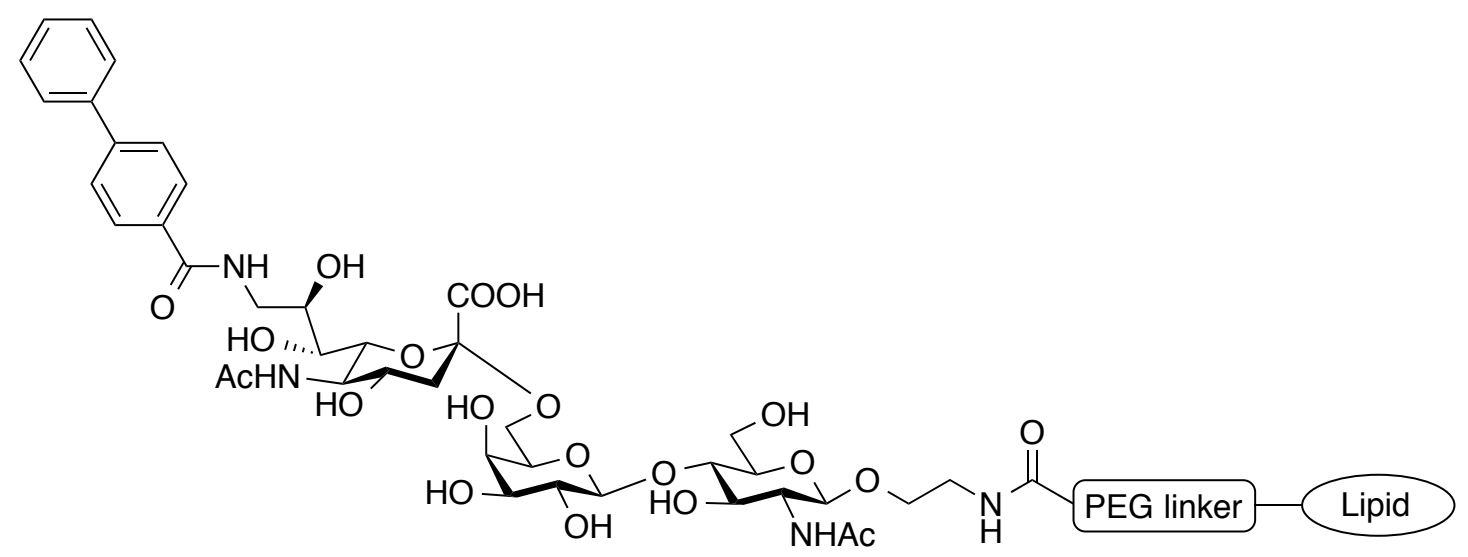

Figure 6. ${ }^{B P C}$ NeuAc conjugated to a PEGylated lipid through an ethylamine linker. ${ }^{32}$

\subsubsection{Labelled Glycoconjugate Probes}

The labelling of glycoconjugates with a chemical "tag" of some description can serve a variety of functions. For example, glycoconjugates armed with a fluorescent tag, such as fluorescein isothiocyanate (FITC), can be used to visualise and identify cells by 
microscopy or flow cytometry. Here, the glycan of the glycoconjugate targets the fluorescent probe to specific cell types due to the cells' unique cell surface receptor expression. Glycoconjugates can also be fluorescently labelled to simply visualise if the conjugate has been incorporated within or on the surface of cells.

Fluorescent glycolipids have also been manufactured to study various aspects of membrane structure. Bertozzi and co-workers prepared a series of glycosylphosphatidylinositol (GPI) analogues and investigated their behaviour in lipid bilayers. ${ }^{34}$ The GPI anchor is a post-translational modification added to many proteins in order to anchor them within the lipid membrane so that the protein itself can reside entirely on the external surface of the cell membrane. Bertozzi's GPI analogues varied between having none, one or two mannose units so as to probe how these systematic deletions affected the behaviour of the GPI-protein analogues in living cells. To synthesise the target glycolipids, a phospholipid was attached to the reducing end of the mannose saccharides via an alkyl chain while native chemical ligation was used to attach green fluorescent protein (GFP) to the GPI anchor via a cysteine functionalised phosphoethanolamine linker. By visualising the movement of these anchors, it was found that increasing the sugar residues on the GPI anchors resulted in more rapid diffusion through the lipid bilayer.

Trifunctional glycoconjugates, for activity-based protein profiling (ABPP), can be used as site-directed molecular probes to assess the activity of glycoside-processing enzymes. The three functionalities include a carbohydrate unit that will bind to a specific target enzyme, a reactive functional group or "trap" which forms a covalent bond with the enzyme after binding, and finally a tag to visualise or purify the bound target enzyme. A linker is often used to spatially separate the trap and tag so that the function of each is not influenced by the other. For example, a trifunctional probe (Figure 7) was used to isolate $\mathrm{N}$-acetylglucosaminidases. Here, $\mathrm{N}$-acetylglucosamine was linked to an $\mathrm{o}$-monofluoromethyl-phenoxy trap, which was tethered to biotin via an amide linker. After binding to the enzyme, the glycosidic bond is cleaved allowing the trap to react with a nucleophilic group in the enzyme's active site. The biotin labelled enzyme can then be isolated using streptavidin-based affinity column chromatography. ${ }^{35}$ 


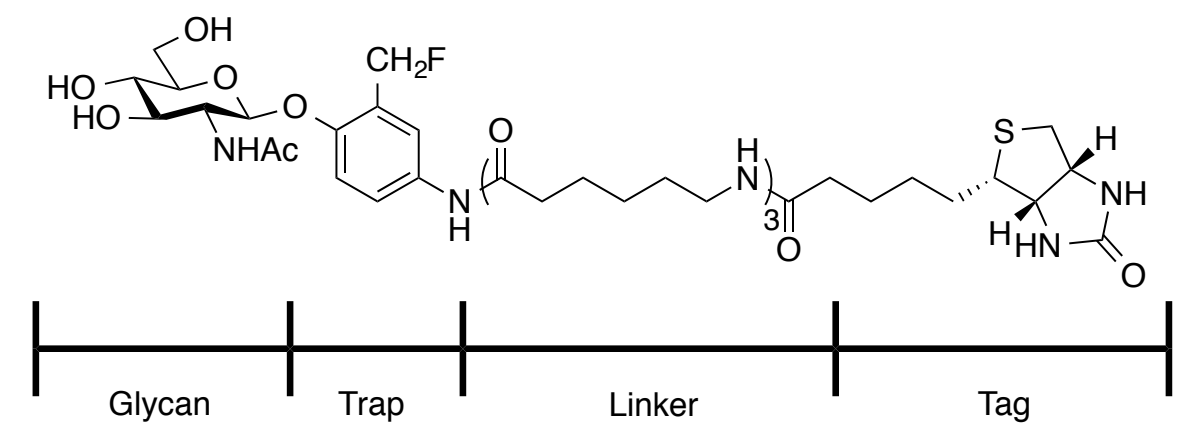

Figure 7. A trifunctional probe used to isolate $N$-acetylglucosaminidases. ${ }^{35}$

\subsection{Conjugation Strategies}

As mentioned above, in order to conjugate a carbohydrate to the substrate of choice, effective and reliable linking strategies are required. This often necessitates the need for a chemical linker. Some current procedures involve carbohydrate protecting group strategies so that the linkers can be installed at the start of glycan total synthesis. ${ }^{14}$ However, this approach is lengthy and does not allow for the use of naturally derived sugars. As a result, a number of different linking methodologies have been developed which allow for the conjugation of all carbohydrates with a free reducing end without the need for protecting groups. Typical strategies involve $N$-glycoside synthesis and include reductive amination, ${ }^{36}$ Kochetkov amination, ${ }^{37}$ the preparation of oximes and hydrazones, and the use of oxyamine linkers (Scheme 1). ${ }^{38}$

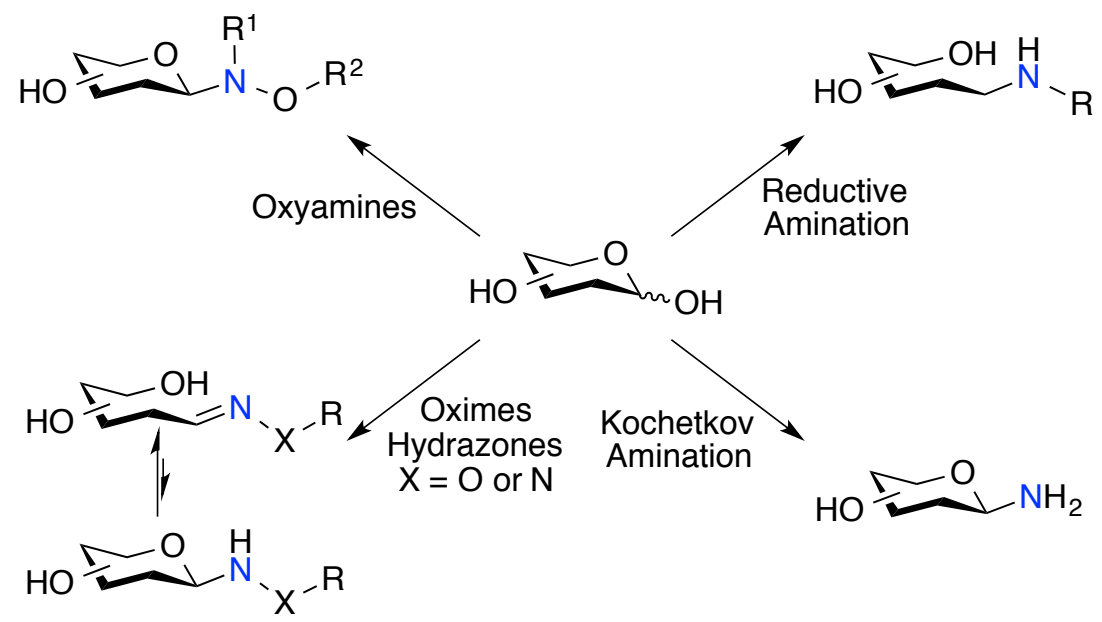

Scheme 1. Ligation methods for free sugars. 


\subsubsection{Reductive Amination}

During reductive amination, the glycan's aldehyde functionality is converted to an imine, which is subsequently reduced by a hydride source to form the corresponding amine. ${ }^{39}$ This technique permanently renders the sugar in its ring-open form, which could influence lectin binding and glycan recognition. Despite this, the use of reductive amination for the preparation of glycoconjugates has often been used, as illustrated by Cummings and co-workers who used reductive amination to rapidly synthesise glycan microarrays for galectin binding studies, ${ }^{36}$ and Gildersleeve and co-workers who used reductive amination to attach high mannose sugars to lysine residues on proteins. ${ }^{40}$

\subsubsection{Kochetkov Amination}

Kochetkov amination enables the formation of $\beta$-D-glycosylamines by reacting a glycan with a free reducing end in an ammonium carbonate solution for over 24 hours at approximately $40{ }^{\circ} \mathrm{C}$. This renders the carbohydrate with a free amine at the anomeric centre. Although this methodology has been elegantly applied, as demonstrated by Danishefsky and co-workers who utilised the free amine functionality to directly link carbohydrates via aspartylation to amino acid residues in their landmark synthesis of erythropoietin $(\mathrm{EPO}){ }^{29}$ the resulting 1-amino glycosylamine Kochetkov amination products are not very stable in solution, which makes them less favourable as intermediates in the synthesis of glycoconjugates. This instability is also related to the functionality and steric interaction of the group attached to the C-2 position of the sugar, which is suggested to be due to stabilising hydrogen bonding interactions between the anomeric amine and the group at the $\mathrm{C}-2$ position. ${ }^{41}$ These 1 -amino glycosylamines also readily undergo anomerisation, as well as trans acylation, when neighbouring acetamido linkages are present. ${ }^{42,43}$

\subsubsection{Hydrazines and Oximes}

The use of hydrazine and oxime ligation strategies for the formation of glycoconjugates can be carried out in aqueous conditions and without the need for protecting groups. ${ }^{38}$ Glycosyl hydrazines and oximes have been used in a variety of biological and analytical applications. For example, Feizi and co-workers developed a bi-functional hydrazide linker bearing both a chromophore and a biotin moiety (Figure 8 ). ${ }^{44}$ The naphthyl group 
could be used as a fluorescence tag for high performance liquid chromatography (HPLC) analyses, while biotin enables the immobilisation of the glycan in microtiter plates for use in enzyme-linked immunosorbent assays (ELISAs). Biotin functionalised glycoconjugates, prepared using a hexanoic acid hydrazide linker, have also been used to immobilise carbohydrates onto streptavidin coated SPR chips in order to investigate lectin-specific binding. ${ }^{30}$ Glycosyl hydrazines and oximes have been used in the synthesis of biologically relevant glycoconjugates, as demonstrated by Peluso and Imperiali who synthesised a novel aspartyl hydrazide amino acid that they incorporated into a peptide mimetic through solid phase peptide synthesis. ${ }^{45}$ The $N$-acetylglucosamine (GlcNAc) was subsequently condensed onto the peptide through the hydrazide moieties, and the resulting glycoconjugate was found to be a potent inhibitor of oligosaccharyl transferase. $^{46}$

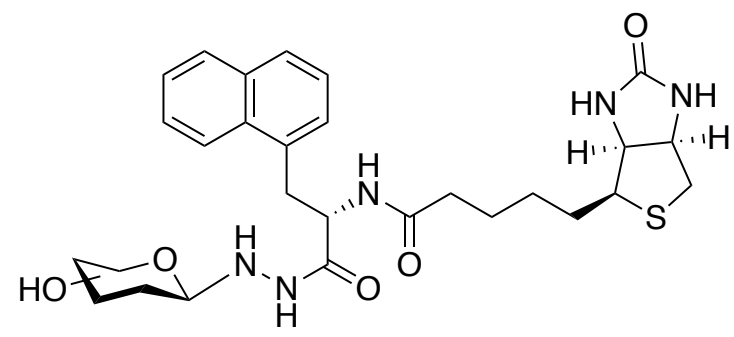

Figure 8. Napthyl and biotin functionalised hydrazine linker developed by Feizi and coworkers, conjugated to an unspecified glycan. ${ }^{44}$

Notwithstanding the number of important applications of glycosyl hydrazines and oximes, it is known that in solution the oxime and hydrazine glycoconjugates form an equilibrium between the ring-opened hydrazone/oxime and the ring-closed pyranosyl and furanosyl forms, ${ }^{47}$ which may affect lectin binding. To overcome this, Peri and coworkers demonstrated that by using $\mathrm{N}, \mathrm{O}$-dialkylated oxyamines, exclusive formation of the ring-closed glycoconjugate is observed, which protects the structural integrity of the sugar. $^{48}$

\section{$1.4 \quad N, O$-Dialkyl Oxyamine Conjugation}

Primary oxyamine glycoconjugates predominantly exist in the ring-opened oxime form in solution with, for example, approximately $70 \%$ of glucosyl oximes being in their ringopened form. ${ }^{49}$ The utility of oxyamine-based glycoconjugates, however, was 
substantially increased with the introduction of an alkyl substituent on the nitrogen, which allows for the exclusive formation of the ring-closed product. ${ }^{50}$ These secondary oxyamines can be coupled to reducing sugars in good yield under mildly acidic conditions. $^{50}$ The concentration of each coupling partner is crucial under aqueous conditions. This is because the equilibrium constant of formation for the reaction (determined in $\mathrm{D}_{2} \mathrm{O}$ at $\mathrm{pD} 4.5$ ) was found to be small (10-20 M). Consequently, only conjugation reactions under high concentrations are able to reach near completion. ${ }^{43}$

Oxyamine linkers can be divided into two sub-types, the "Type A" $O$-alkyl- $N$-methyl oxyamines and the "Type B" $N$-alkyl-O-methyl oxyamines (Scheme 2). Both types have been reported and successfully conjugated to carbohydrates by way of a bi-functional linker. However, the "Type A" oxyamine linkers are more widely used and have been studied more extensively.

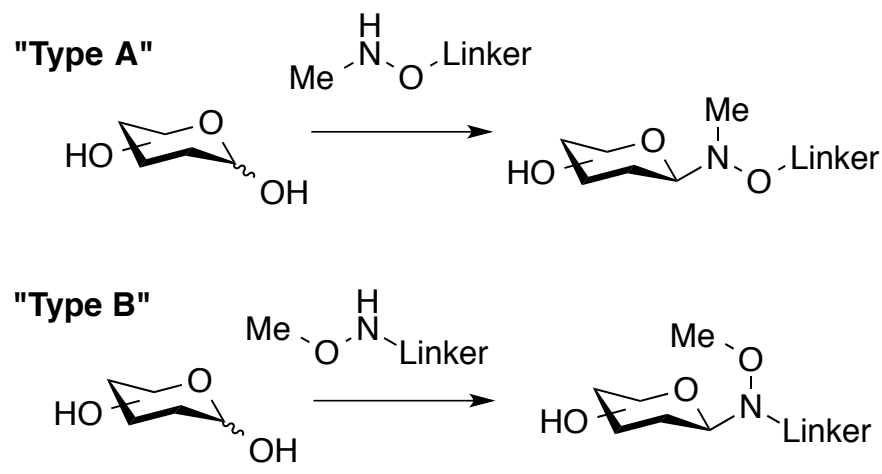

Scheme 2. "Type A" and "Type B" oxyamine glycoconjugates.

"Type A" bi-functional oxyamine linkers were initially reported by Bohorov et al. for use in carbohydrate microarrays. ${ }^{51}$ This 5 -step linker synthesis began with $N$-Boc protection of ethanolamine (1) followed by mesylation and subsequent displacement using lithium bromide to form bromide 2 (Scheme 3). $N$-Methylhydroxylamine was also $N$-Boc protected to form $N$-Boc- $N$-methylhydroxylamine (3), which was then alkylated to bromide 2 , followed by Boc deprotection to form the TFA salt of the bi-functional linker 4 in $8 \%$ overall yield. The authors were then able to conjugate linker 4 to various naturally derived carbohydrates in high yields, as exemplified in Scheme 4 with the coupling of $N$ acetyl-lactosamine (LacNAc). The glycoconjugates were then immobilised onto NHS functionalised glass microarray slides and subsequently used to screen lectin and antibody 
binding. In 2014, Boons and co-workers also used the bifunctional linker 4 with an Fmoc group attached to the terminal amine. The linker was conjugated to lactose prior to enzymatic extensions to create more complex polysaccharides. The Fmoc group provided a chromophore for UV detection and provided the polysaccharides with enough lipophilic character to enable purification by HPLC using a reverse phase column. ${ }^{52}$

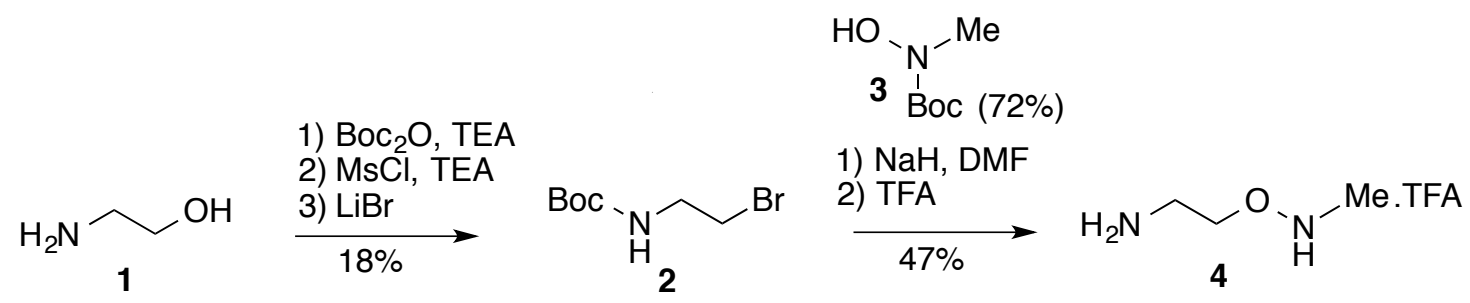

Scheme 3. Bohorov's synthesis of "Type A" oxyamine linker $4 .{ }^{51}$

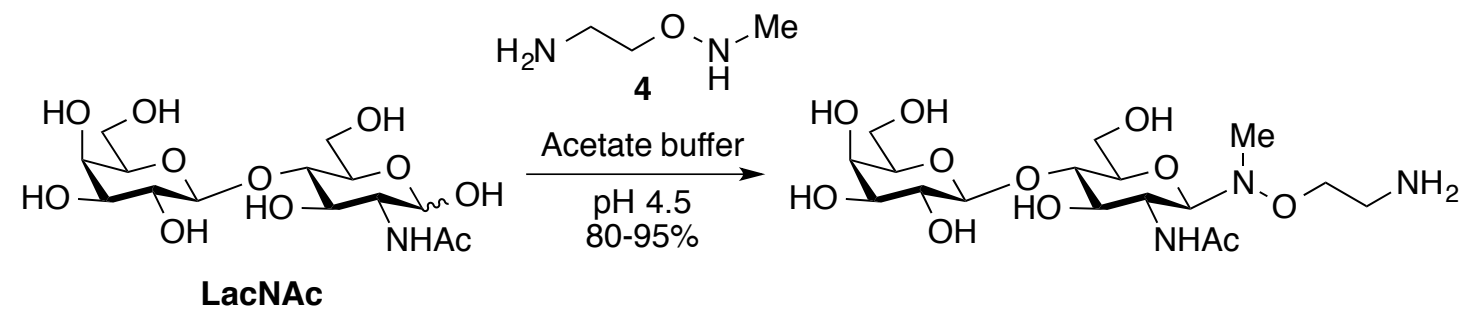

Scheme 4. Conjugation of the disaccharide, LacNAc to "Type A" linker 4.

“Type B" bi-functional oxyamine linkers have been utilised to a lesser degree in the literature. Recently, Munneke et al. demonstrated that bi-functional "Type B” oxyamine linkers can be made rapidly in a 3-step-1-pot synthesis with excellent overall yields (80$96 \%) .{ }^{53}$ The linker synthesis begins with acrolein (5), which functions as a bi-functional reagent (Scheme 5). Acrolein is reacted with $\mathrm{NaN}_{3}$ in a Michael addition followed by condensation with methoxyamine to generate an intermediate imine, which was then reduced with $\mathrm{NaCNBH}_{3}$ to produce linker 6 . The versatility of this method lies in the use of acrolein as the starting material, which enables a number of different functionalities to be incorporated at the end of the alkyl chain. A representative "Type B" linker was then conjugated to a number of glycans under mildly acidic conditions in excellent yields, as illustrated via conjugation to Lewis ${ }^{\mathrm{x}}(88 \%)$ (Scheme 6). 


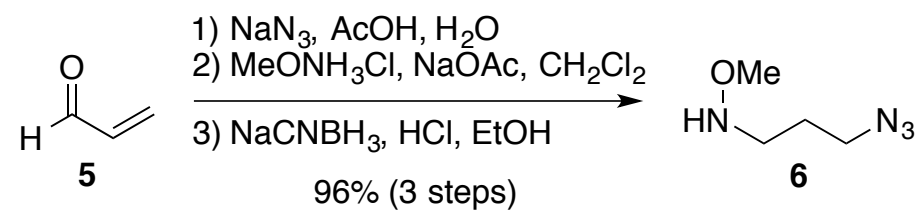

Scheme 5. Munneke's synthesis of "Type B" oxyamine linker $6 .{ }^{53}$

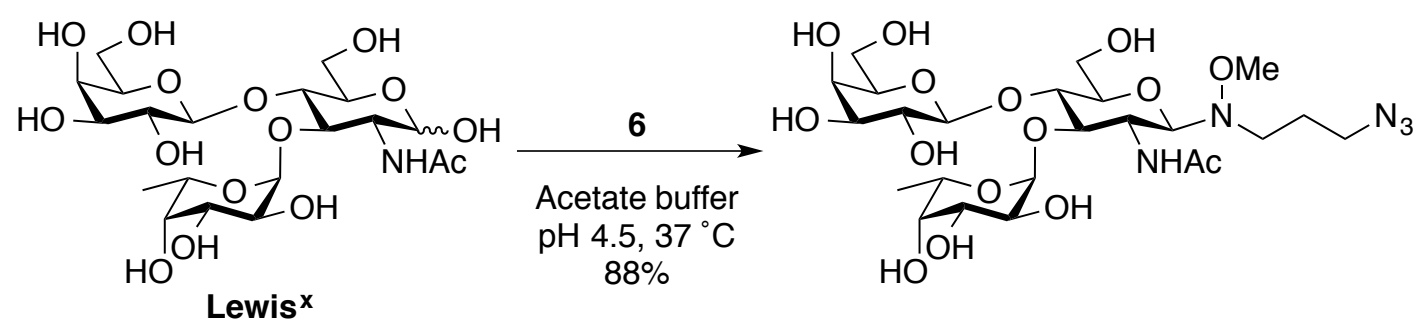

Scheme 6. Conjugation of oxyamine linker 6 to Lewis ${ }^{x}{ }^{53}$

\subsection{Scope and Limitations of Oxyamine Glycan Conjugation}

The mechanism of oxyamine glycosylation involves the formation of an intermediate oxyiminium ion. Accordingly, it is possible to form mixtures of $\alpha$ - and $\beta$-pyranosides or furanosides of the oxyamine-linked carbohydrate, whereby the exact isomeric distribution depends largely on the steric influence of the substituents on the immediate saccharide unit. The reversibility of this reaction means that the product distribution is governed by the relative thermodynamic stability of each isomer rather than any kinetic influences. Langenhan et al. carried out a detailed investigation on these isomeric distributions using model benzyl oxyamine aglycones and simple monosaccharides. ${ }^{50}$ Glucose derivatives exclusively formed $\beta$-pyranose products, galactose derivatives yielded $\beta$-pyranose/ $\beta$-furanose mixtures, while mannosides afforded a mixture of the $\beta$ pyranose/ $\alpha$-pyranose/ $\alpha$-furanose forms (Figure 9 ). The exclusive formation of $\beta$-pyranose products for the glucosides can be explained by the unfavourable steric interactions between the C-3 and C-4 groups of the furanosides. The all-equatorial configuration of $\beta$-gluco-pyranose minimises 1,3-diaxial strains, while any stabilising anomeric effect presented by the oxyamine for the $\alpha$-pyranoside anomer is not sufficient to overcome the steric disadvantage of having an axial substituent. The $\beta$-pyranoside isomer was also the predominant product when forming galactosides. However, the $\beta$-furanoside was formed in minor amounts ( $c$. 35\%), which is presumably due to the lack of any disfavoured steric interactions between the C-3 and C-4 groups for this isomer. Again, the absence of 
any galactose $\alpha$-anomers is likely to be due to an insufficient oxyamine anomeric effect. Mannosides gave a 2:2:1 mixture of the $\alpha$ - and $\beta$-pyranosides to the $\alpha$-furanoside. Here, the disfavoured presence of two axial substituents on the $\alpha$-pyranoside is offset by the favoured opposing C-1-C-2 dipoles and possibly a weak anomeric effect. It is important to note that in addition to the pyranoside isomers, the $\alpha$-furanoside also makes a minor contribution ( $c a .20 \%$ ) to the equilibrium mixture of the mannosides, despite steric hindrance between the C-3 and C-4 substituents. It thus appears that there is some stabilising effect from the C-1-C-2 opposing dipoles in the $\alpha$-furanose configuration.

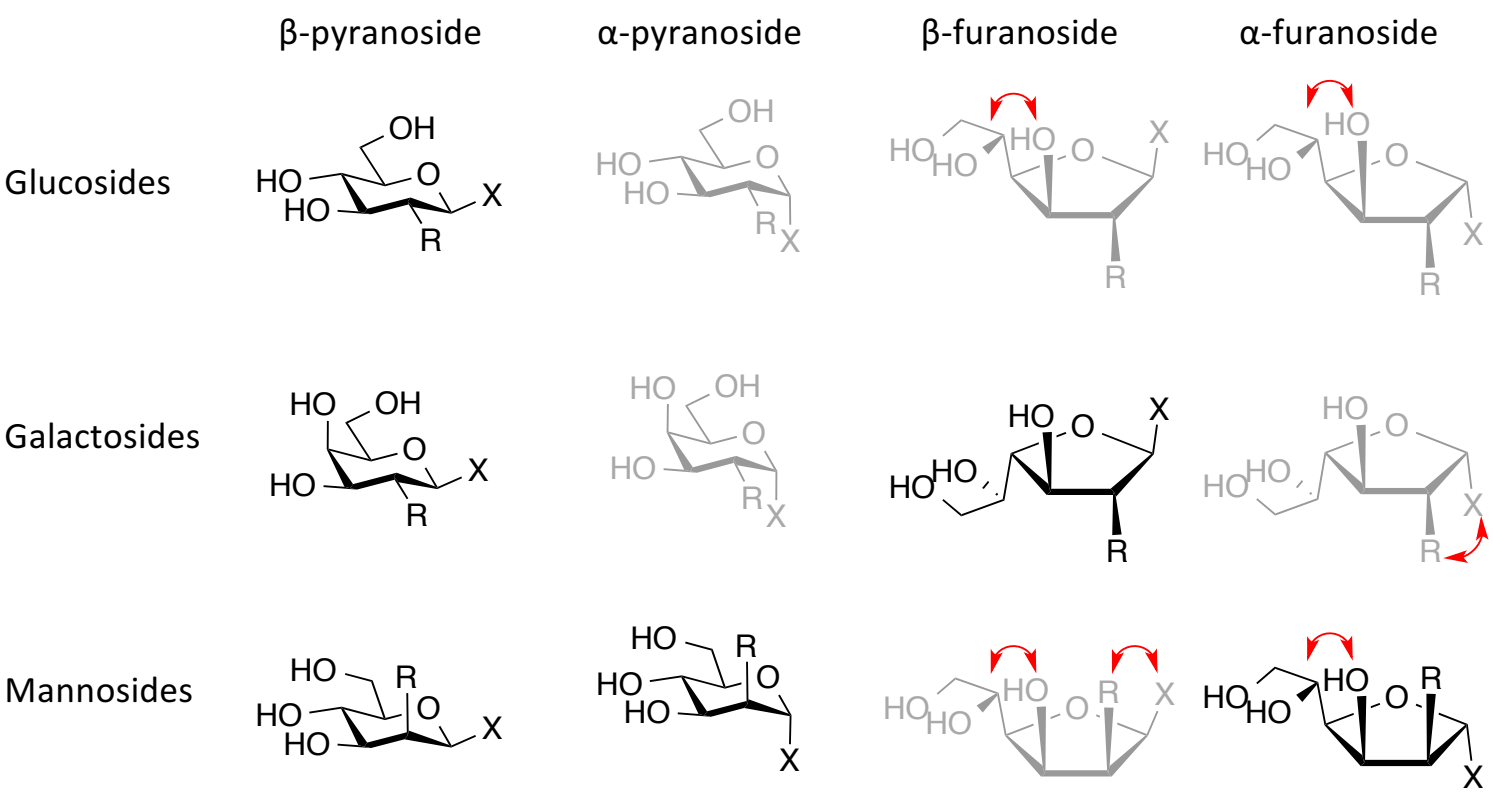

Figure 9. Isomer formation in the glycosylation reaction for both "Type A" and "Type $B$ " oxyamine linkers. ${ }^{50}$ The isomers in grey are not observed and the red arrows indicate steric strain.

\section{$1.6 \quad \mathrm{~N}, \mathrm{O}$-Alkyl- $\mathrm{N}$-Glycosyl Oxyamines in Synthesis}

\subsubsection{Glycoprotein Synthesis}

Peri et al. utilised "Type A" $N$-methyloxyamine functionalised amino acids in order to synthesise novel glycoprotein mimics. ${ }^{48}$ In this work, a lysine residue was acylated with a protected $O$-oxyamine, followed by deprotection to yield the oxyamine functionalised peptide 7. $\mathrm{N}$-Methylation was then achieved via reductive amination with formaldehyde and $\mathrm{NaCNBH}_{3}$. Subsequent ligation to a variety of glycans (e.g. maltotriose) under mildly 
acidic conditions produced the desired glycoprotein mimic 8 in good yield (Scheme 7). $\mathrm{N}$-Linked glycoproteins, however, are naturally conjugated to serine or threonine amino acids, and to better mimic these naturally occurring glycosides, studies towards the synthesis of methoxyamine functionalised serine and threonine amino acids were undertaken. ${ }^{54,55}$ To this end, Carrasco et al. prepared both "Type A" and "Type B" methoxyamine amino acids of serine and threonine (9a, 9b and 10, Figure 10) and incorporated them into SPPS to form oxyamine peptides. These amino acids were then glycosylated with glucose under mildly acidic conditions with the exception of the threonine residue. The inability of oxyamine-threonine $\mathbf{1 0}$ to react was likely due to the steric hindrance imposed by the methyl substituent.

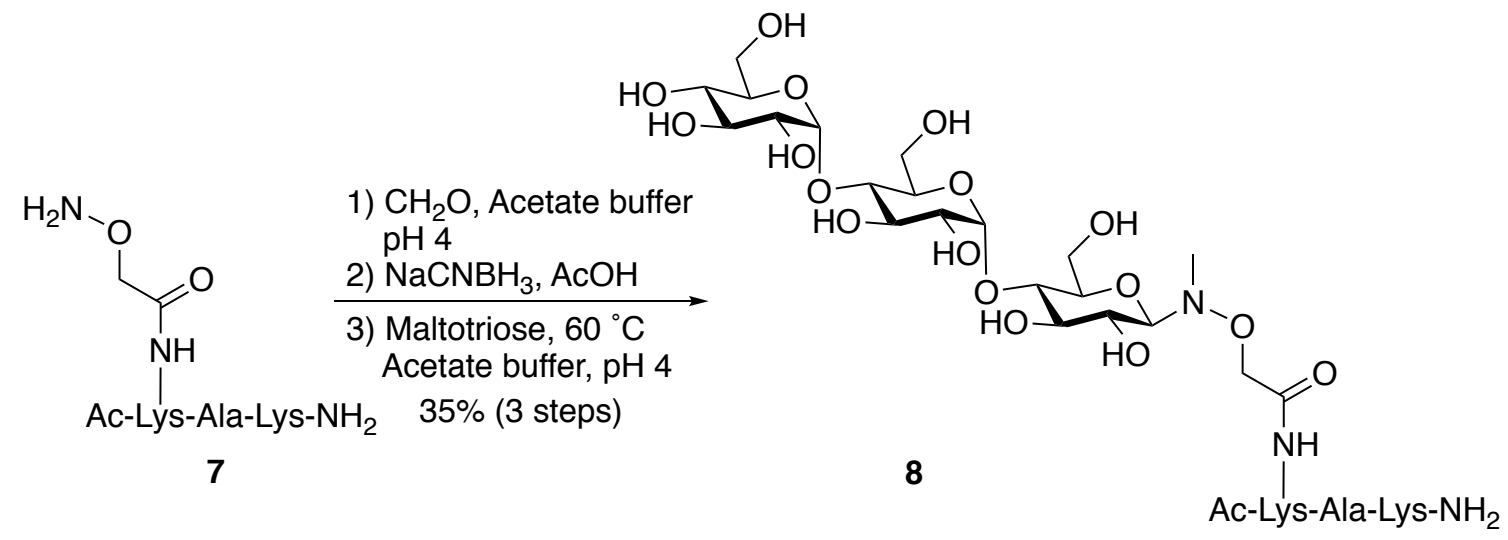

Scheme 7. Use of oxyamine functionalised peptide 7 for the synthesis of glycopeptide mimics. $^{48}$<smiles>CONC[C@H](N)C(=O)O</smiles>

$9 a$<smiles>CNOCC[C@H](N)C(=O)O</smiles>

$9 b$<smiles>CON[C@@H](C)[C@H](N)C(=O)O</smiles>

10

Figure 10. Oxyamine functionalised serine (9a), homoserine (9b) and threonine (10) analogues. ${ }^{54,55}$

\subsubsection{Oligosaccharide Mimetics}

It is also possible to form oligosaccharides by exploiting oxyamines as inter-glycosidic linkages. The ease of formation of the $N$-glycosyloxyamine linkage and the predictability of the diastereoselective outcome of the reaction means that oxyamines have great 
potential to produce oligosaccharide mimetics rapidly and efficiently. Peri and coworkers explored the use of "Type B" methoxyamine functionalised glucose in the formation of $\beta 1 \rightarrow 6$ linkages for the preparation of di- and tri-saccharides. ${ }^{56,57} \mathrm{~A}$ methoxyamine substituent was installed at the 6-position of glucose (11) through DessMartin oxidation of the 6-OH and subsequent reductive amination with methoxyamine (Scheme 8). The resulting methoxyamine functionalised glucose 12 was then conjugated to a variety of monosaccharides to yield disaccharide mimetics 13. Methoxyamine glucose 12 can also be condensed with 6-methyloxime-functionalised monosaccharides 14 in an iterative process to generate trisaccharide 15 (Scheme 9). Due to the formation of $\alpha / \beta$-pyranosyl/furanosyl isomeric mixtures for non-glucosides, this conjugation method is best suited to the coupling of glucose monosaccharides.

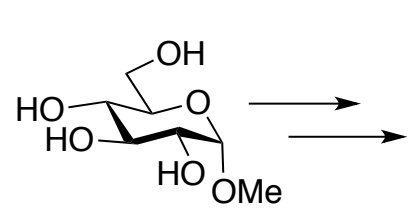

11

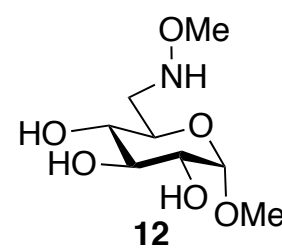

12
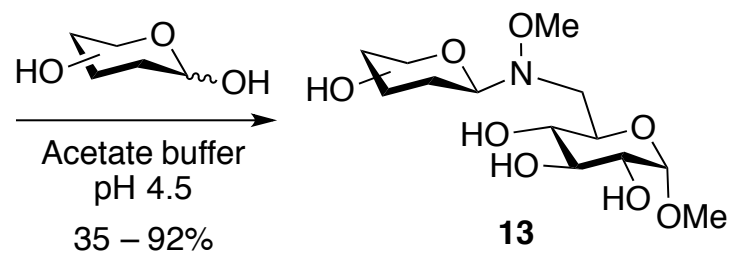

Scheme 8. Synthesis of N-linked oxyamine disaccharide mimic. ${ }^{57}$

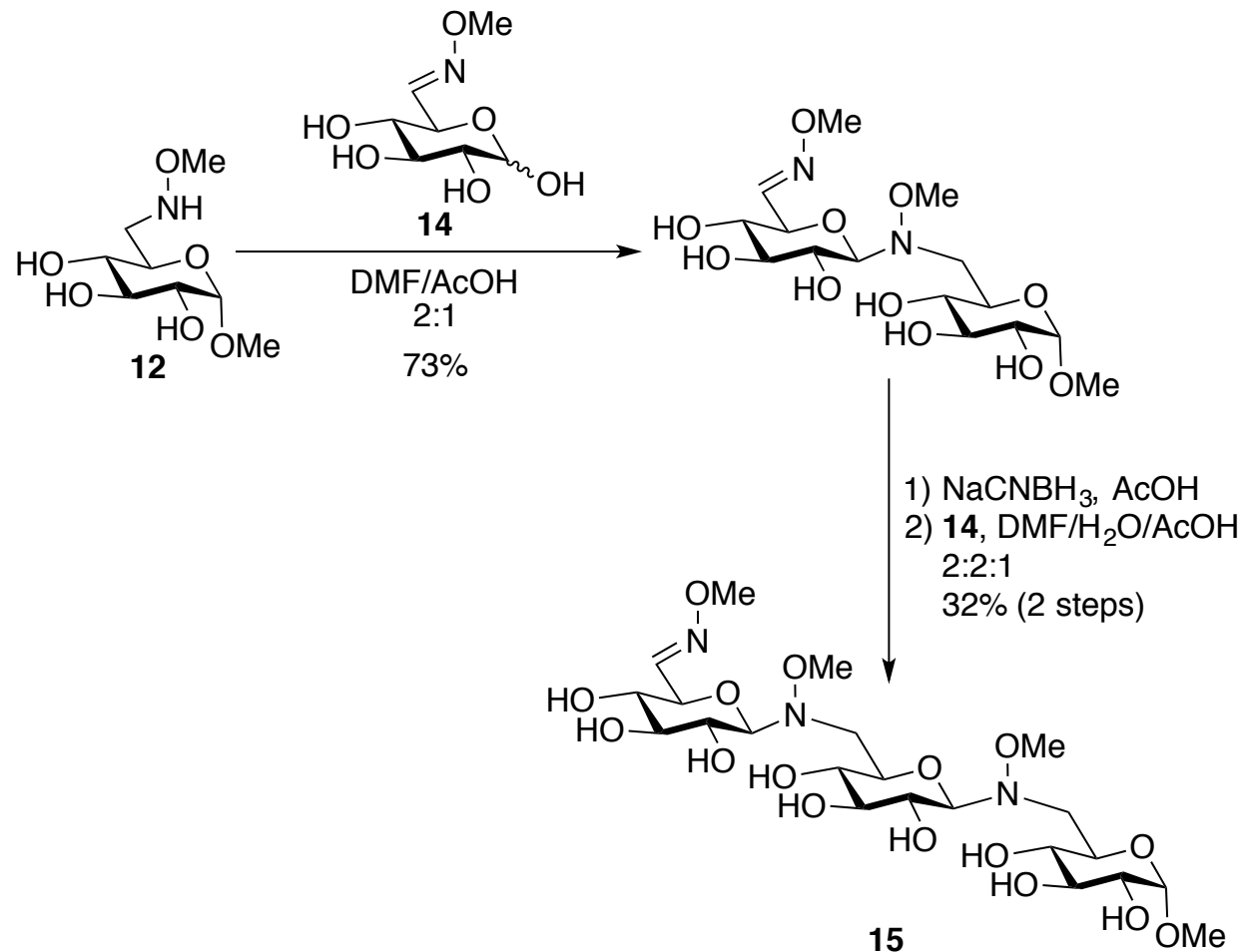

Scheme 9. Synthesis of N-linked oxyamine trisaccharide mimic. ${ }^{56}$ 


\subsubsection{Neoglycorandomisation}

The objective of neoglycorandomisation is the production of a library of neoglycosylated compounds which only differ in the structure of the attached sugar (Scheme 10). Langenhan and co-workers used "Type B" oxyamine conjugation strategies as a method of rapidly glycosylating natural products to produce a library of reducing sugars in order to create novel glycoconjugates which may have higher biological activity than the naturally derived digitoxin glycoconjugate. ${ }^{58}$ The first target for neoglycorandomisation was digitoxin, a glycosylated cardenolide (type of steroid) with potential anticancer activity. ${ }^{59}$ The cardenolide backbone of digitoxin was modified to contain an $\mathrm{N}$ alkyloxyamine moiety (16), which allowed for rapid glycosylation to reducing sugars to produce a 78-member library (Scheme 11). This library was then tested in a highthroughput cytotoxicity assay against nine human cancer cell lines to assess if any particular glycan increased the efficacy and cell specificity of the conjugated drug. It was found that the L-ribose glycoside of digitoxin displayed much higher potency compared to digitoxin alone while the D-mannose conjugate displayed excellent selectivity towards the multidrug-resistant NCI/ADR-RES cells. Since then, several bioactive probes have been neoglycosylated in this way to enhance their activity or to discover potential new drugs. Examples include betulinic acid, ${ }^{60}$ cyclopamine, ${ }^{61}$ warfarin, ${ }^{62}$ vanomycin $^{63}$ and colchicine, ${ }^{64}$ amongst others. ${ }^{65}$

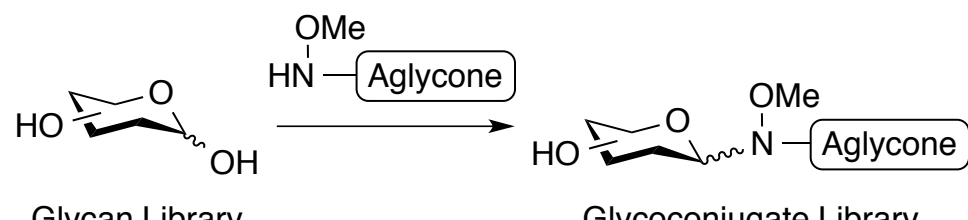

Scheme 10. Neoglycorandomisation. 


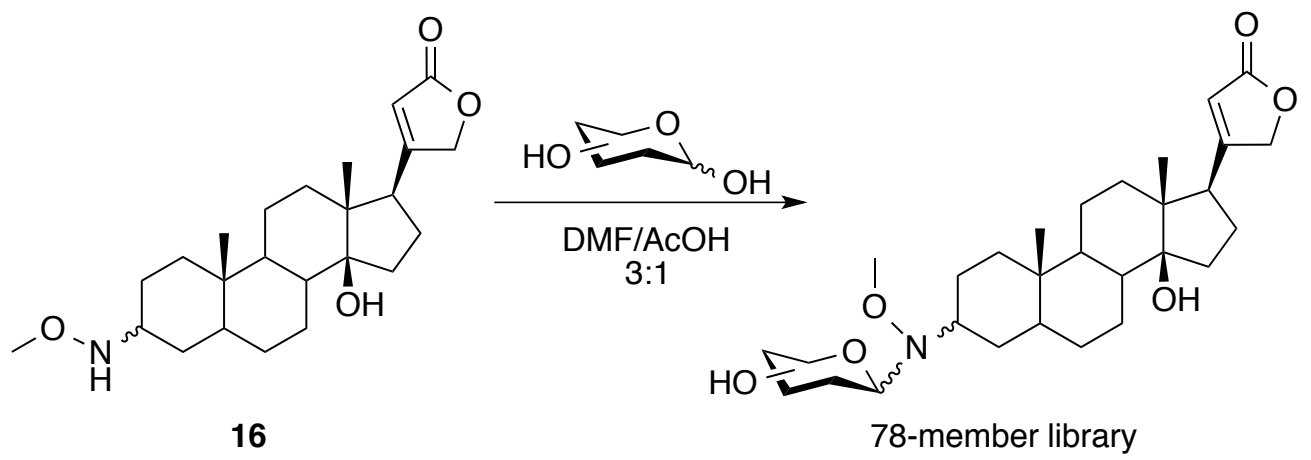

Scheme 11. Neoglycorandomisation of digitoxin. ${ }^{59}$

\subsection{Oxyamine Glycoside Stability}

The stability of oxyamine glycosides to aqueous hydrolysis is an important aspect to keep in mind when using these glycoconjugates for medicinal applications. The acidic equilibrium conditions under which the glycosidic bonds form means that their hydrolytic cleavage can readily take place in conditions below $\mathrm{pH} 7$. To this end, the hydrolytic stability of both "Type A" and "Type B" oxyamine glycosides has been assessed, 43, 50, 53, ${ }^{66}$ however as side-by-side studies using identical reaction conditions (e.g. $\mathrm{pH}$ and/or glycan concentration) were not conducted, it is difficult to determine whether one type of oxyamine glycoside was more stable than the other. The independent studies, however, indicated that the hydrolysis of the oxyamine glycoside is pseudo-first order, highly $\mathrm{pH}$ dependent, and that the rate of hydrolysis is highly dependent on the electronics of the substituents on the nitrogen, the oxygen, and the sugar. ${ }^{38,43,50,53,66}$ These studies also suggested that the mechanism of hydrolysis is consistent with that of a specific acidcatalysed (SAC) process, however only limited evidence has been provided. Conjugates formed from electron rich sugars and/or electron rich oxyamines hydrolyse significantly faster than those bearing electron poor substituents. ${ }^{66}$ This is rationalised by the ease of protonation of the electron rich species. For example, xylose-containing glycoconjugates $\left(t_{1 / 2}=29 \mathrm{~h}\right)$ were much less stable than glucose glycoconjugates $\left(t_{1 / 2}=71 \mathrm{~h}\right)$, which in turn were less stable than GlcNAc-containing glycoconjugates $\left(t_{1 / 2}=1100 \mathrm{~h}\right)$, when submitted to a buffer of $\mathrm{pH} 6{ }^{43}$ It has also been determined that substituents on the nitrogen of the oxyamine have a greater effect on the conjugate stability than substituents on the oxygen. ${ }^{66}$ For example, $N$-benzyloxyamines hydrolyse more slowly $\left(t_{1 / 2}=168 \mathrm{~h}\right.$ ) than the equivalent $N$-methyloxyamine conjugate $\left(t_{1 / 2}=34 \mathrm{~h}\right)$ when subjected to a buffer 
of $\mathrm{pH} 4{ }^{66}$ It is important to note, however, that $N$-acetylglucosamine derivatives displayed excellent hydrolytic stability at physiological $\mathrm{pH}(\sim 7.4){ }^{53}$

\subsection{The Application of $N, O$-Dialkyl Oxyamine Linkers for the Multivalent Display of Chitin Fragments}

\subsubsection{Chitin}

Chitin is a polymer of $\beta$-1,4-linked $N$-acetyl-D-glucosamine (GlcNAc) (Figure 11), and is the second most abundant polysaccharide in nature after cellulose. Chitin is absent in mammals, but is an essential component of the cell walls of fungi, the exoskeletons of crustaceans and insects, the microfilarial sheath of parasitic nematodes, and within the lining of the digestive tract of many insects. ${ }^{67-70}$ The presence of human chitinases suggests a first line of defence against chitin-containing parasites and is also indicative of chitin recognition mechanisms, whereby chitin is recognised as a pathogen associated molecular pattern (PAMP). Previous studies have demonstrated that chitin fragments are recognised by the PRRs TLR-2, dectin-1 and the mannose receptor (MR) on innate immune cells such as macrophages and DCs. ${ }^{71,72}$

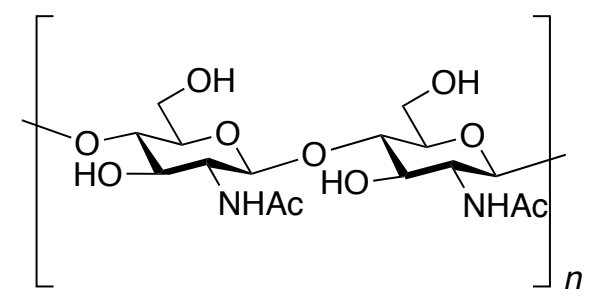

Figure 11. Structure of chitin.

Since the discovery of chitin's ability to activate the immune system, chitin has been found to elicit T-helper 1 (Th1), ${ }^{71,73,74} \mathrm{Th} 2^{75,76}$ and $\mathrm{Th} 17^{72}$ mediated immune responses. When either of these immune responses are activated, the others are down-regulated to ensure only one type of response prevails. ${ }^{77}$ This variable immune response to chitin appears to be dependent on the size of the chitin fragment. ${ }^{78}$ Other factors such as the chitin source, the route of administration and the ability of chitin to be phagocytosed also seem to influence the type of immune response. ${ }^{79}$ Chitin has also been implicated in the allergic asthma immune response (Th2) perpetuated by exposure to chitin sources in the 
home environment, such as from the house dust mite (D. pteronyssinus) and ubiquitous fungi. ${ }^{80}$

There is much interest in the development of Th1 inducing chitin as a potential antiasthma vaccine candidate to force the immune system to recognise chitin as Th1stimulating rather than Th2-stimulating. ${ }^{74}$ However, before this can be achieved, the exact conditions under which chitin is Th1-inducing or Th2-inducing needs to be defined. Previous studies have observed that small chitin particles $(2-10 \mu \mathrm{m})$ elicit an antiinflammatory Th2 response, medium sized particles $(40-70 \mu \mathrm{m})$ stimulate a proinflammatory Th1 response, and smaller soluble particles $(<2 \mu \mathrm{m})$ as well as large, unphagocytosable particles $(70-100 \mu \mathrm{m})$ have no immune stimulatory effects. ${ }^{78}$ The authors rationalise these findings by suggesting that different sized chitin particles play different roles in the regulation of the antiparasite/pathogen response. The large chitin particles are unphagocytosable and therefore do not initiate an immune response via macrophage interactions. The medium sized chitin particles represent a potential threat from a parasite/pathogen, thereby stimulating a pro-inflammatory response. The small chitin particles could be a representation of a pathogen which has already been largely destroyed, thereby providing a mechanism to down-regulate the immune system and stimulate an anti-inflammatory response to prevent excessive tissue damage. Unfortunately, these studies only used mixtures of chitin with various size distributions that was generated from chitin powder. As a consequence, the exact particle size and exposed chitin chain length required for binding and activation are unknown. Accordingly, it is important to more precisely understand the conditions required for the generation of the Th1 and Th2 responses, particularly given the interest in chitin as a vaccine against asthma. To this end, it was envisioned that specific chitin oligomers could be attached to gold nanoparticles (AuNPs) of a specific size using a thiol functionalised oxyamine linker. By testing the immune response of every chitin oligomer length/nanoparticle size combination, the exact conditions for the Th1 and Th2 immune responses could then be determined.

\subsubsection{Gold Nanoparticles for the Multivalent Display of Antigens}

To assess how the structure and size of chitin particles stimulates immune cells, different lengths of chitin oligomers need to be displayed on a multivalent display scaffold to 
produce a particle of a specific, measurable size. Gold nanoparticles offer advantages over other multivalent display scaffolds, such as proteins, dendrimers or polymers for a number of reasons. Gold nanoparticles are relatively easy to synthesise and purify and their size and shape can be finely controlled. ${ }^{81,82}$ They are also stable once the ligands have been attached, ${ }^{83}$ and the density and type of ligand on the surface of AuNPs can be readily varied. ${ }^{81}$ This property can be utilised, for example, to fluorescently label NPs harbouring an antigen, ${ }^{84}$ or to attach an adjuvant and an antigen to the same NP for vaccine purposes. ${ }^{81}$ Moreover, AuNPs are often water soluble and biocompatible for in vivo applications. ${ }^{84}$ To synthesise the functionalised AuNPs, the NPs are prepared in the presence of glycoconjugates bearing a terminal thiol (or disulfide linkage) ${ }^{24}$

\subsection{Project Aims}

This thesis focuses on the development of bi-functional $\mathrm{N}, \mathrm{O}$-dialkyloxyamine linkers for the synthesis of glycoconjugates. To this end, the first objective in this thesis is to improve the synthesis of the "Type A" ( $O$-alkyl- $N$-methyl) oxyamine linkers. Next, studies into the relative hydrolytic stability of the "Type A" and "Type B" ( $N$-alkyl-O-methyl) oxyamine linked glycoconjugates will be undertaken. While studies in the literature have assessed the hydrolytic stability of glycosyloxyamines, the majority focused on the "Type A" oxyamine conjugates and none directly compare the stability of "Type A" vs. "Type B" conjugates. In order to gain a better understanding of the mechanism of hydrolysis of these oxyamine-linked glycoconjugates, the $\mathrm{p} K_{\mathrm{a}}$ of the conjugate acid of these glycosyloxyamines will be determined. This will give insight into the conditions under which these conjugates are stable and, when combined with the hydrolysis studies, may provide further insight into the rate determining step (RDS) of the mechanism.

Finally, the more hydrolytically stable oxyamine linker will then be used to make a sulfur functionalised oxyamine for the multivalent display of glycans on gold nanoparticles. These sulfur-functionalised oxyamine linkers will be conjugated to chitin fragments and then to different, specific sized gold nanoparticles. Although beyond the scope of this thesis, it is anticipated that these glycosylated gold nanoparticles will then be tested for their ability to generate T-helper immune responses. 


\section{Chapter 2}

\section{Synthesis and Hydrolytic Stability of $N$ - and $O$ - Methyloxyamine Linked Glycoconjugates}

\subsection{Introduction}

Carbohydrates play an essential role in the function of a variety of biomolecules. Glycans conjugated to lipids or proteins are known as glycolipids and glycoproteins respectively. It is understood that over $50 \%$ of all proteins are glycoproteins. ${ }^{2,3}$ Glycolipids and glycoproteins are expressed on the surface of a variety of different cell types, these include red blood cells and cells of the immune system, such as leukocytes. These cell surface glycoconjugates have important biological roles. For example, they enable cellcell communication, the locomotion of cells through tissue, and the adhesion of cells to each other to keep tissue intact. ${ }^{85}$ Carbohydrates bound to proteins can also significantly alter the protein tertiary structure, aiding in correct protein folding and enzymatic activity. ${ }^{7}$ Due to the large diversity of carbohydrate structures, the function of the same protein can vary depending on the structure or number of carbohydrates attached. ${ }^{86}$ Thus, in order to understand the effect of glycan structure on protein or lipid function, the synthesis of well-defined glycoconjugates in the form of glycan microarrays, ${ }^{17,} 18$ glycoconjugate probes, ${ }^{21}$ glycoproteins ${ }^{13}$ and multivalent glycan displays is required. ${ }^{19}$ The most efficient strategy for the synthesis of such glycoconjugates involves conjugation to the reducing end of the carbohydrate, which avoids the need for protecting groups and is therefore amenable to both synthetic and naturally formed carbohydrates. Ligation strategies include Kochetkov amination, ${ }^{37}$ reductive amination ${ }^{36}$ and the use of oximes, hydrazones and oxyamines. ${ }^{38}$

Ligation through oxyamines has proven to be particularly advantageous as it provides the glycoside in good yield while maintaining the integrity of the reducing end sugar by preserving the ring-closed chair configuration. ${ }^{38,48}$ In addition, oxyamine-linked glycosides show good hydrolytic stability under biological conditions. ${ }^{43,53}$ There are two sub-types of oxyamine linkers which are used for the conjugation of glycans: the "Type A" $O$-alkyl- $N$-methyl oxyamines and the "Type B" $N$-alkyl-O-methyl oxyamines 
(Scheme 12). To date, the "Type A" linker has been more widely used and extensively studied. Multiple strategies for the synthesis of the "Type A" linker have been reported. ${ }^{29}$, 51,52 However, such strategies have low overall synthetic yields (8-25\%) and require 5-6 synthetic steps. Additionally, these methods require different approaches for different terminal functional groups. Consequently, there is a need for the improvement of the synthesis of the "Type A" linker to create a more rapid and efficient methodology. More recently, an efficient and adaptable synthesis of a bifunctional "Type B" linker has been published by Munneke et al. (Scheme 13). ${ }^{53}$ Munneke's elegant 3-step-1-pot synthesis can be used to make multiple different oxyamine linkers which vary in their terminal functional groups. ${ }^{53}$
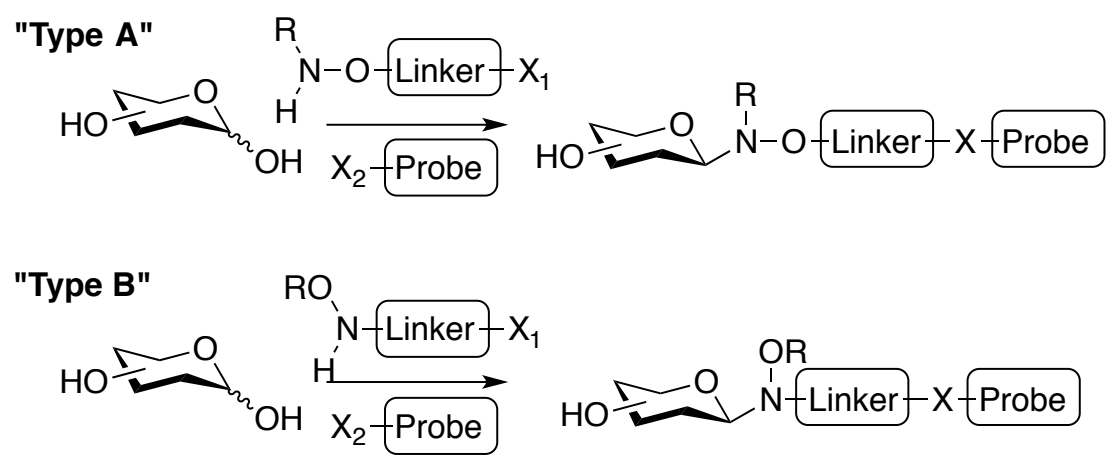

Scheme 12. "Type A" and "Type B" oxyamine glycoconjugates.

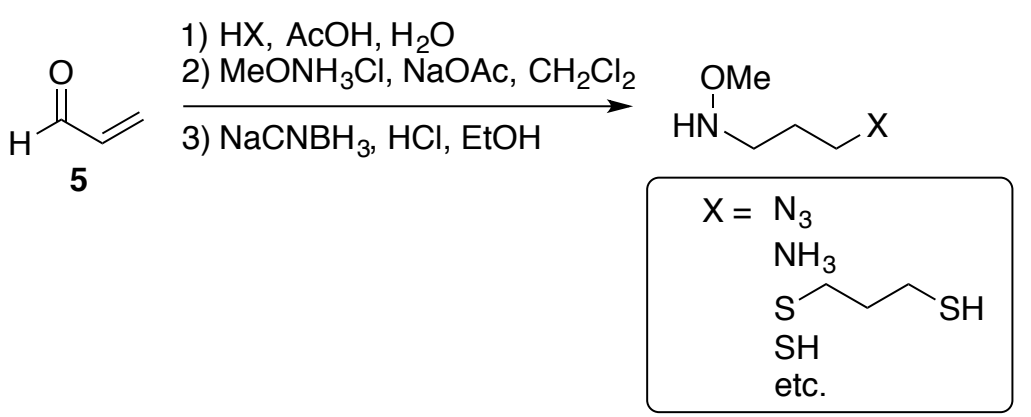

Scheme 13. Munneke's synthesis of "Type B" oxyamine linkers. ${ }^{53}$

An important aspect of the chemical properties of glycosyl oxyamines is their hydrolytic stability. It is essential to know the conditions under which these glycosides are stable, particularly, whether they are stable at the $\mathrm{pH}$ and molar concentrations relevant for biological applications. As mentioned in the introduction of this thesis, previous hydrolytic stability studies have been conducted on "Type A" glycosyl oxyamines. ${ }^{38,43}$, 50, 53, 66 These studies concluded that the hydrolysis of glycosyl oxyamines is highly $\mathrm{pH}$ 
dependent and a pseudo first-order process. They also determined that the hydrolysis reaction undergoes specific acid-catalysis and that electron poor oxyamines and/or saccharides provide more stability towards hydrolysis. Cló et al. investigated a variety of glucosyl oxyamines. ${ }^{66}$ They found that the rate of hydrolysis of glucosides with an anomeric $N$-benzyl substituent (Figure 12A) were four- to five-fold slower than those bearing an anomeric $\mathrm{N}$-methyl substituent (Figure 12B) in the same $\mathrm{pH}$ range. This corresponds to the observation that more electron poor oxyamines yield more hydrolytically stable glycoconjugates. They also investigated glucosyl oxyamines which vary in their $O$-alkyl substituent. It was noted that only a marginal increase in hydrolytic stability was observed for the $O$-benzyl $v s$. the $O$-methyl conjugates at the same $\mathrm{pH}$. This agrees with other studies, including those performed by Langenhan et al., ${ }^{50}$ which also found that varying the $O$-alkyl substituents only has minor effects on the hydrolytic stability of glycosyl oxyamines. This observation has been rationalised through the mechanism of hydrolysis. The nitrogen atom is directly involved in the formation of the oxyiminium ion, which is thought to occur before the rate determining step. ${ }^{43}$ As a consequence, the nitrogen substituents will have a larger effect on the ease of formation of the oxyiminium ion and thus the rate of hydrolysis.

A

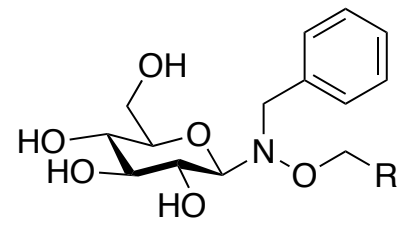

B

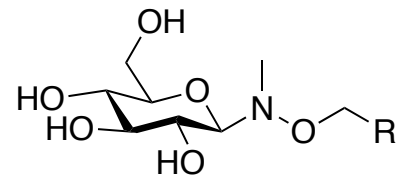

Figure 12. A) N-benzylglucosyloxyamine. B) N-methylglucosyloxyamine. ${ }^{66}$

Glycan and linker concentration influences the rate and extent of glycoconjugate formation, ${ }^{43}$ and accordingly, it is likely that the concentration of these reagents also influences the hydrolysis rate. The effect of glycoconjugate concentration on hydrolytic stability has not been studied with regard to oxyamine glycoconjugates, although it has been suggested that more dilute concentrations of these glycoconjugates favours hydrolysis. ${ }^{38}$ In addition, the buffer molarity in previous hydrolytic studies has varied between experiments. It is not known how this affects the rate of hydrolysis.

While valuable insight into oxyamine hydrolysis has been obtained, none of these studies have directly compared the stability of glycosides formed from the "Type A" oxyamine 
linkers to those formed from the "Type B" variety. Accordingly, the focus of this chapter concerns the first direct comparison between the merits of the "Type A" and "Type B" linkers for the formation of glycosides. To undertake this study, an improved synthesis of the "Type A" linker was performed, and then differences in the hydrolytic stability between similar "Type A" and "Type B" glycosyl oxyamines in response to $\mathrm{pH}$, glycoside concentration, and buffer strength, was explored.

\subsection{Results and Discussion}

\subsubsection{Optimisation of "Type A" Linker Synthesis}

"Type A" bi-functional oxyamine linkers were first reported by Bohorov et al. for use in carbohydrate microarrays (Scheme 14). ${ }^{51}$ Bohorov's procedure involved the $N$-Boc protection of ethanolamine (1) followed by mesylation of the hydroxyl and then reaction with $\mathrm{LiBr}$ to afford $\mathrm{N}$-Boc protected bromoethylamine 2. $\mathrm{N}$-Methylhydroxylamine was also $N$-Boc protected to give $\mathbf{3}$ before alkylation with $\mathbf{2}$ followed by Boc deprotection using TFA to produce the bi-functional "Type A" oxyamine linker 4 (Scheme 14). While this "Type A" linker is very useful for the preparation of a variety of glycoconjugates, the route suffers from a very low $(8 \%)$ overall yield. A more recent procedure described by Prudden and Boons et al. utilised a Mitsunobu reaction to generate the Fmoc protected version of Bohorov's linker in $34 \%$ overall yield (Scheme 15). ${ }^{52}$ During this synthesis, ethanolamine (1) was reacted with Fmoc chloride to provide intermediate 18, which was treated with 3 under Mitsunobu conditions to afford the protected linker 19. Boc deprotection was achieved with $4 \mathrm{M} \mathrm{HCl}$ in dioxane to give Fmoc-protected linker 20. However, as illustrated, this synthetic strategy does not include Fmoc deprotection. This is because the authors used the Fmoc group as a chromophore for UV/Vis detection to monitor subsequent reactions by HPLC. The Fmoc group also enabled the linker to be more lipophilic for the convenience of purification by reverse phase chromatography. 


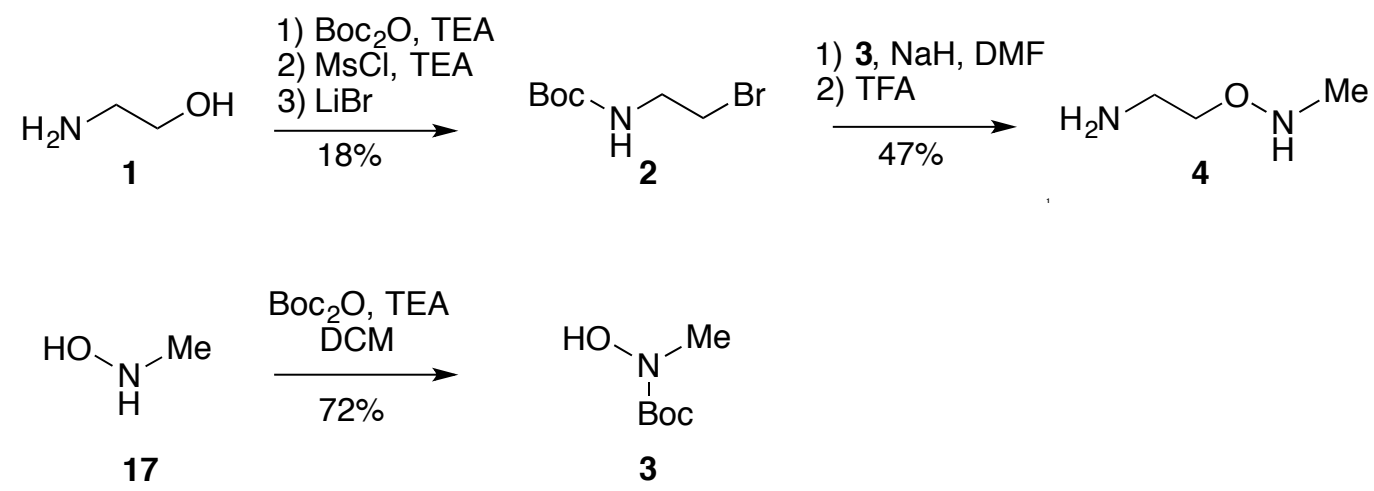

Scheme 14. Bohorov's synthesis of "Type A" oxyamine linker $4 .{ }^{51}$

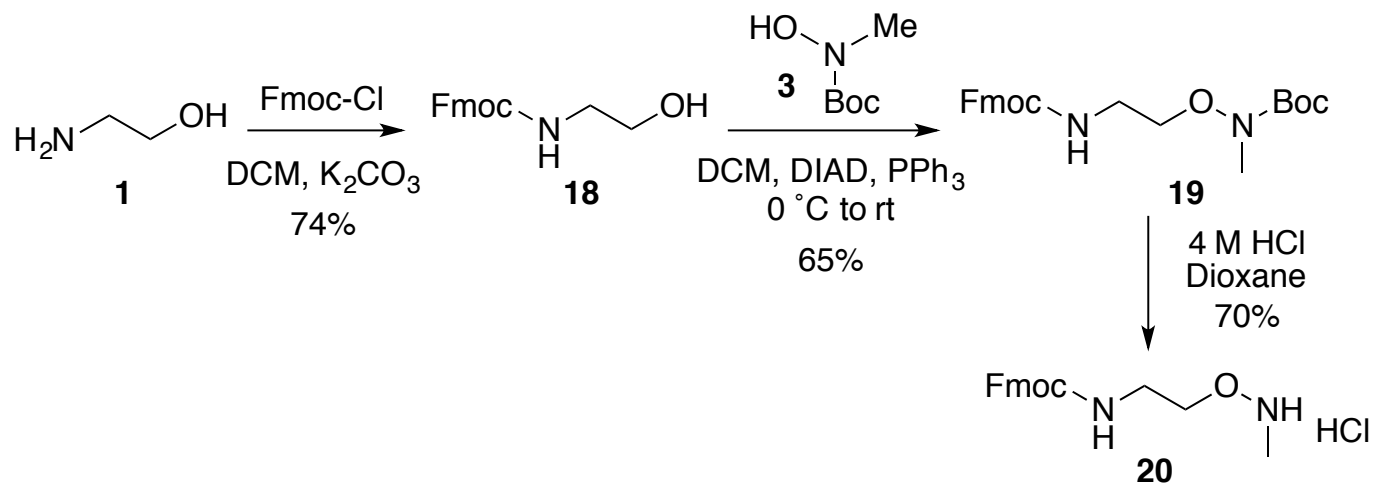

Scheme 15. Prudden and Boons' synthesis of Fmoc-protected "Type A" oxyamine linker $20 .^{52}$

Accordingly, it was envisioned that a more efficient synthesis of the "Type A" oxyamine linker 4 could be achieved via a modification to Bohorov's route by reducing the number of protection/deprotection steps in the synthesis. First, an attempt was made to react bromoethylamine hydrobromide (21) with $\mathrm{N}$-methylhydroxylamine hydrochloride (17) with no protecting group chemistry (Scheme 16A). This alkylation was attempted in DMF using $\mathrm{NaH}$, as was used to couple $\mathbf{2}$ and $\mathbf{3}$ together in Scheme 14. An aqueous workup using an organic solvent to extract the product was not possible due to the polar nature of the product, instead the solvent of the reaction mixture was evaporated in vacuo, however, only starting materials were recovered. Solubility was also an issue, with the reaction mixture being relatively insoluble in DMF. An attempt was then made to react unprotected bromoethylamine hydrobromide (21) with Boc-protected $\mathrm{N}$ methylhydroxylamine (3), also using $\mathrm{NaH}$ in DMF (Scheme 16B). The reaction mixture was extracted with ethyl acetate to provide only starting materials. 
A

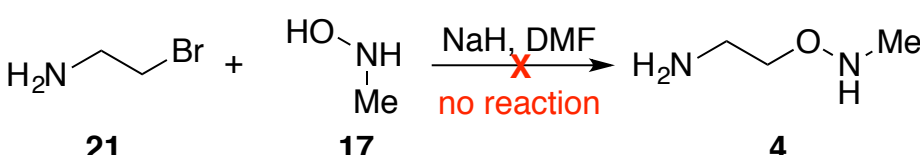

B

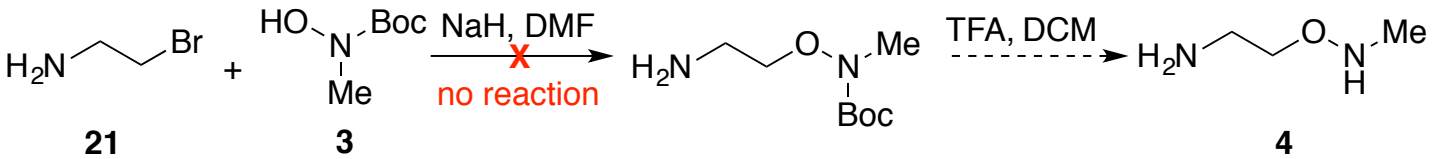

Scheme 16. Attempted syntheses of 4 using fewer protecting groups.

An attempt was then made to synthesise linker 4 starting the synthesis with bromoethylamine hydrobromide (21, Scheme 17) instead of ethanolamine (1, Scheme 14) and using a minimum number of protecting group manipulations. This strategy is advantageous as it eliminates two synthetic steps involved in transforming the alcohol into a bromine functionality in Bohorov's synthesis. In this new approach, 2bromoethylamine hydrobromide (21) was treated with di-t-butylpyrocarbonate $\left(\mathrm{Boc}_{2} \mathrm{O}\right)$ to give $N$-Boc protected bromoethylamine (2) in excellent yield (89\%), as compared to Bohorov's original 3 -step synthesis (18\% yield) for this common intermediate. $N$-Boc protected bromoethylamine (2) was then condensed with Boc-protected $\mathrm{N}$ methylhydroxylamine (3), which itself was formed from $N$-methylhydroxylamine (17) in good yield (Scheme 17), to provide the Boc-protected linker. The protected linker was then treated with TFA to afford the "Type A" linker 4 in four steps and with an excellent overall yield of $38 \%$.
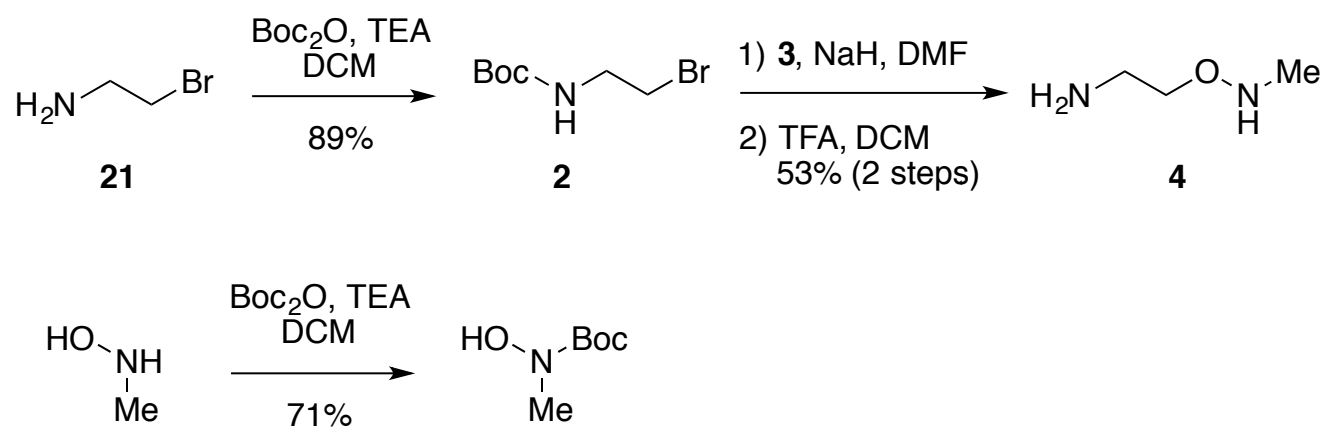

17

3

Scheme 17. Improved synthesis of the "Type A" linker 4. 


\subsubsection{Hydrolytic Stability Studies}

To determine the factors that influence the hydrolytic stability of "Type A" and "Type B" oxyamine glycoconjugates, equivalent "Type A" and "Type B" linkers conjugated to a simple monosaccharide were synthesised. Their hydrolytic stability was then compared by varying $\mathrm{pH}$, conjugate concentration and molarity of the phosphate buffer in which the reaction takes place.

\subsubsection{Conjugate Synthesis}

To compare the hydrolytic stability of the two different types of oxyamine linkers, the stability of the "Type A" linker $\mathbf{4}$ was compared to the stability of the analogous "Type B" linker 22, which was reported by Munneke et al. ${ }^{53}$ (Figure 13). The "Type B" linker 22 was prepared by S. Munneke according to his own published route. ${ }^{53}$ Here, conjugate addition of sodium azide to acrolein (5), followed by condensation with methoxyamine and reduction of the imine intermediate with sodium cyanoborohydride produced azide functionalised linker 6 in a 3-step-1-pot procedure (Scheme 18). Staudinger reduction of intermediate $\mathbf{6}$ then provided 22 in excellent yield (79\%, two steps).
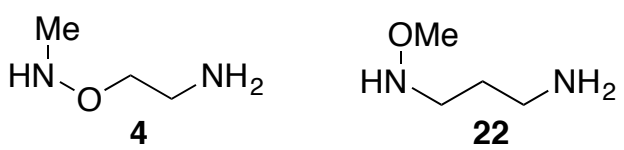

Figure 13. Similar "Type A" and "Type B" oxyamine linkers.

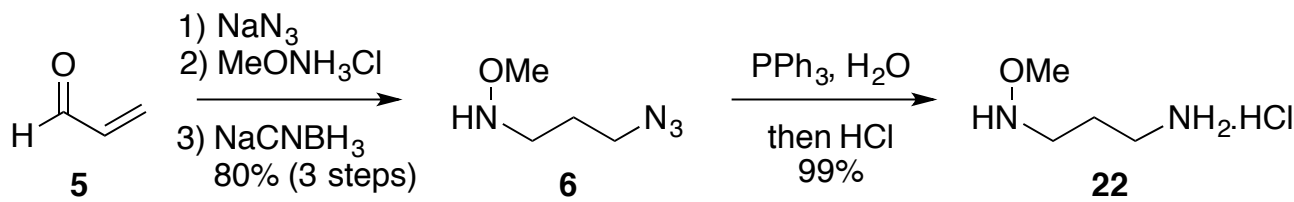

Scheme 18. Munneke's synthesis of "Type B" oxyamine linker $22 .{ }^{53}$

Both representative linkers $\mathbf{4}$ and $\mathbf{2 2}$ were then conjugated to $\mathrm{N}$-acetylglucosamine (GlcNAc). GlcNAc was chosen because it is a glucose derivative and will thus exclusively form the $\beta$-pyranose conjugation product, as described in the introduction of this thesis. This sugar is also biologically relevant as all naturally occurring $N$-linked glycoproteins are conjugated through a GlcNAc residue to the rest of the glycan structure. Conjugation was achieved in an acetate buffer at pH $4.5(2 \mathrm{M})$ with a GlcNAc 
concentration of $0.1 \mathrm{M}$ to provide the "Type A" glycoconjugate $\mathbf{2 4}$ and the "Type B" glycoconjugate 25 in $83 \%$ and $81 \%$ yields, respectively (Scheme 19). The exclusive formation of the $\beta$-pyranose product was confirmed by ${ }^{1} \mathrm{H}$ NMR spectroscopy, with the only anomeric proton resonance exhibiting a ${ }^{3} J_{1,2}=9.7$ and $9.8 \mathrm{~Hz}$ for 24 and 25 respectively (Figure 14). This relatively large value is typical for 1,2-trans-diaxial coupling constants in $\beta$-pyranosides. ${ }^{87}$ The conjugation was also confirmed by HMBCs between the NMe protons of the linker and the anomeric carbon of GlcNAc for $\mathbf{2 4}$ (Figure $15)$, and between the $\mathrm{N}-\mathrm{CH}_{2}$ protons of the linker and the anomeric carbon for 25 .

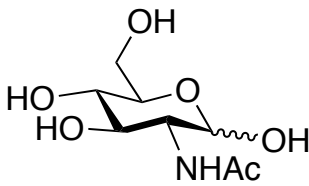

23

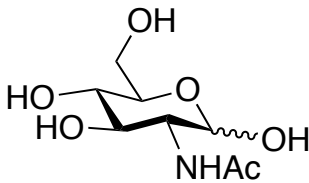

23

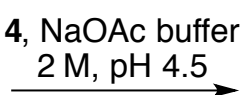

rt, 24 h, $83 \%$

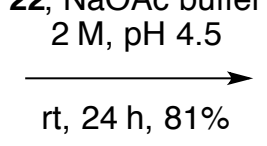

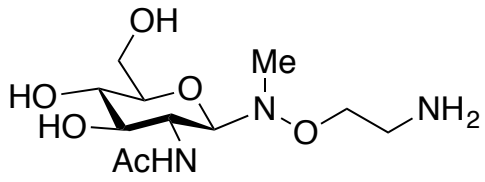

24

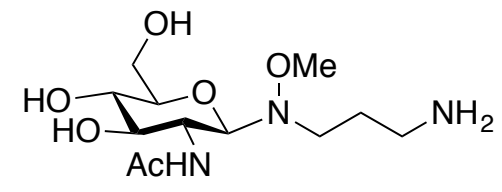

25

Scheme 19. Conjugation of GlcNAc (23) to both "Type A" and "Type B" oxyamine linkers.

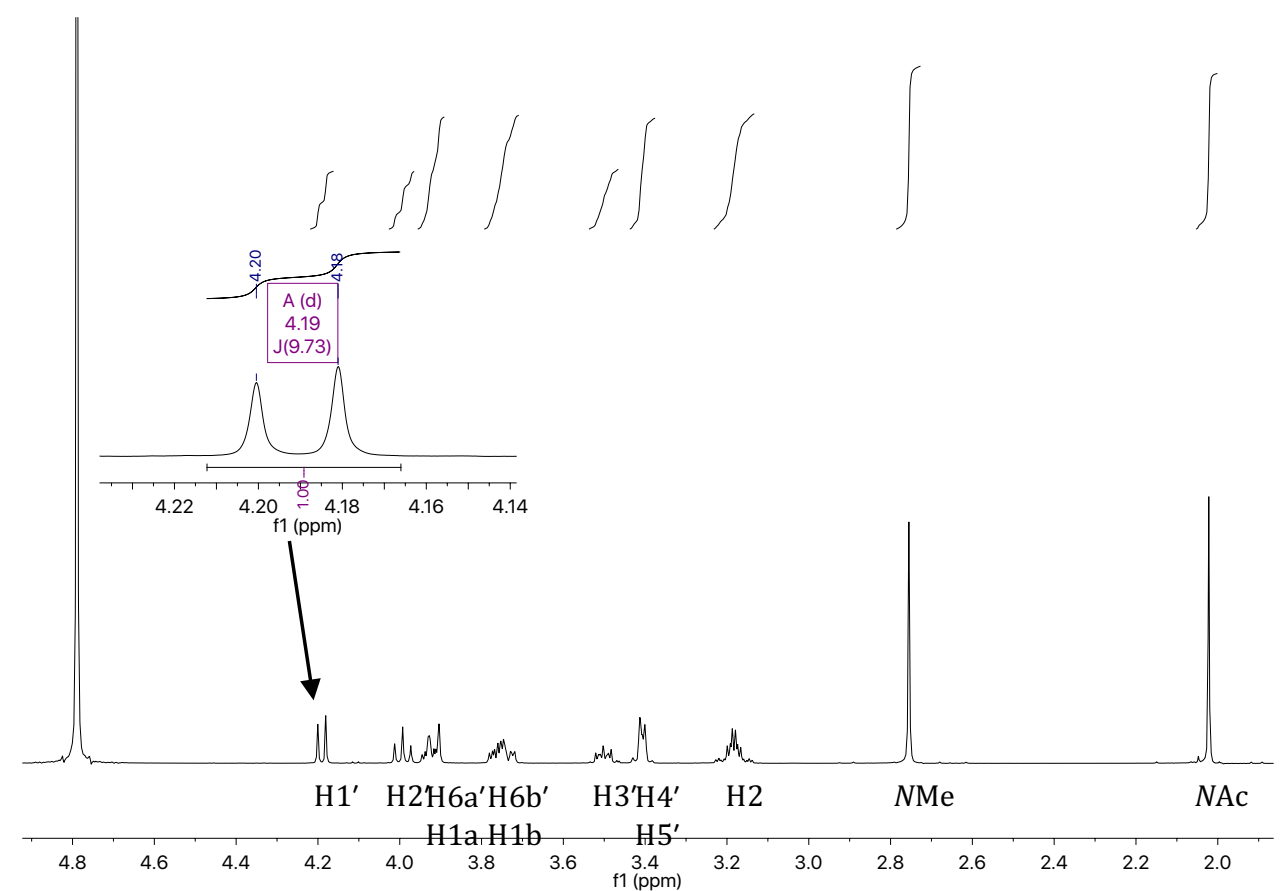

Figure 14. ${ }^{1} \mathrm{H} N \mathrm{NR}$ spectrum for $24\left(\mathrm{D}_{2} \mathrm{O}, 500 \mathrm{MHz}\right)$. Insert illustrates the coupling constant of the anomeric proton. 


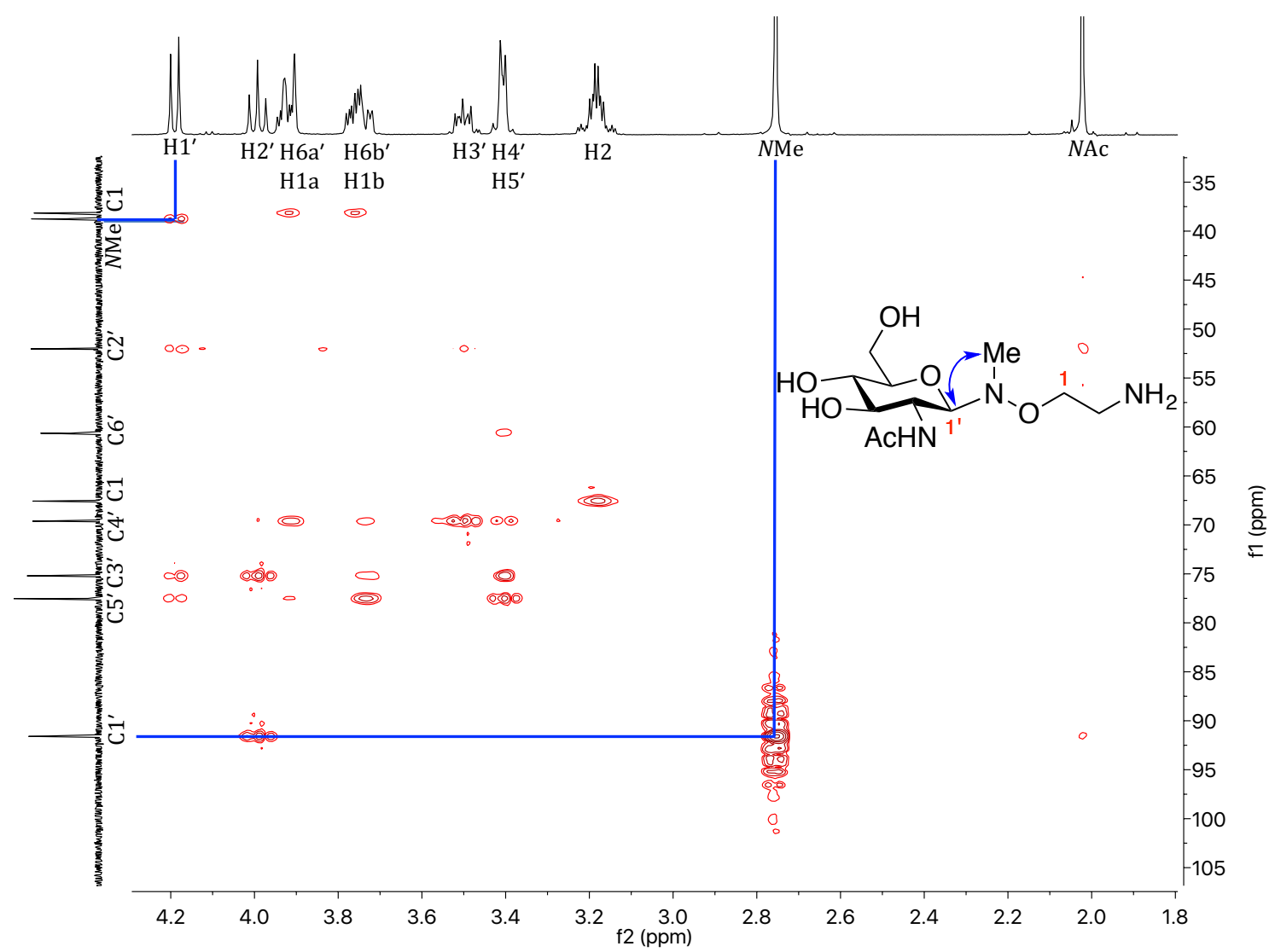

Figure 15. $\mathrm{HMBC}$ for conjugate $24\left(\mathrm{D}_{2} \mathrm{O}, 500 \mathrm{MHz}\right)$.

\subsubsection{Measuring the Rate of Hydrolysis of Glycosyloxyamines}

Both ${ }^{1} \mathrm{H}$ NMR spectroscopy and HPLC have been utilised to measure the rate of hydrolysis of oxyamine glycoconjugates in previous hydrolytic stability studies. ${ }^{43,53}$ The advantage of using ${ }^{1} \mathrm{H}$ NMR spectroscopy is that there is no need to further functionalise the glycoconjugates with a fluorophore as is required for HPLC measurements. To measure the rate of hydrolysis using ${ }^{1} \mathrm{H}$ NMR spectroscopy, appropriate proton resonances from the starting material and/or degradation products are integrated and measured against the integral of a proton resonance belonging to an internal standard. ${ }^{43}$, ${ }^{88}$ The decrease in starting material or increase in degradation products measured over time, relative to the internal standard, allows the rate of hydrolysis to be calculated. However, HPLC is a more accurate and reliable method for the measurement of the relative amounts of the glycoconjugates and their degradation products than ${ }^{1} \mathrm{H}$ NMR spectroscopy. $^{43,88}$ 


\subsubsection{Measuring Rate of Hydrolysis: Initial Design Using ${ }^{1} H$ NMR Spectroscopy}

Initially, ${ }^{1} \mathrm{H}$ NMR spectroscopy was used to measure the rate of hydrolysis of the "Type A" and "Type B" glycosides (24 and 25) in a manner similar to the previous study on the hydrolysis of a "Type B" glycoside undertaken by Munneke et al. ${ }^{53}$ Accordingly, ${ }^{1} \mathrm{H}$ NMR spectroscopy was used to measure the proton resonances of the starting material (glycoconjugate, 24), as well as the resonances of the degradation products (free linker, 4 and GlcNAc, 23).

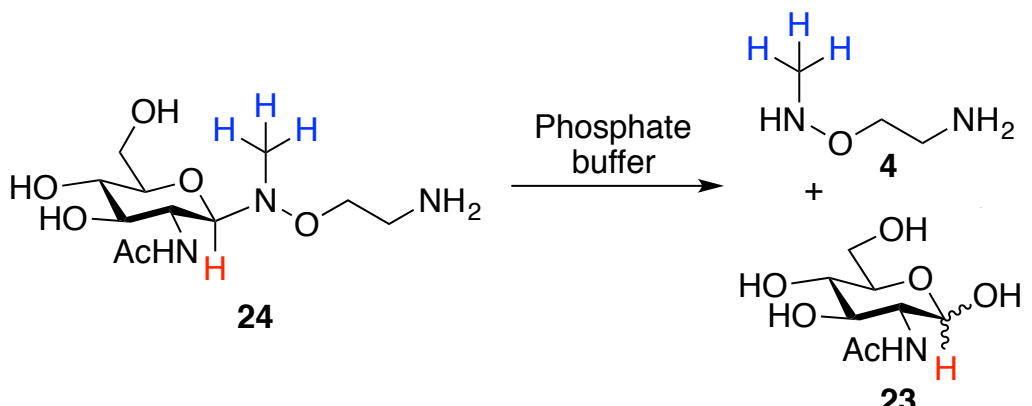

Scheme 20. Hydrolysis of the "Type A" glycoconjugate illustrating the proton signals of importance for measurements using ${ }^{1} H$ NMR spectroscopy.

The $N$-methyl resonance for the "Type A" oxyamine glycoconjugate (Scheme 20 in blue) (or methoxy resonance for the "Type B" glycoconjugate) was chosen as a reference proton signal against which to measure the rate of hydrolysis. This is because the chemical shift of the methyl resonance is very similar when part of the glycoconjugate (24) as well as when part of the free linker (4) (2.75 vs. 3.00 ppm for 24 and free linker 4 respectively). This similarity means that throughout the experiment, the integral of the methyl resonance will be representative of the total amount of glycoconjugate starting material at time $=0$. The anomeric proton resonance (Scheme 20 in red) on the glycoconjugate has a significantly different chemical shift (4.19 ppm for 24) to the anomeric resonance of the GlcNAc degradation product 23 (5.17 and 4.68 ppm for $\alpha$ and $\beta$-GlcNAc respectively). As a result, the integral of the anomeric resonance belonging to the glycoconjugate starting material was measured relative to the integral of the reference proton resonance, taking the 1:3 integral ratio into account. As the glycoconjugate hydrolysed, its anomeric resonance decreased relative to the reference 
proton resonance, this decrease was measured over time so that the rate of hydrolysis could be calculated.

The basic nature of the amine group present on glycoconjugates $\mathbf{2 4}$ and 25 allowed for the deprotonation of acidic compounds it encountered leading to salt formation. It was noted that both $\mathbf{2 4}$ and $\mathbf{2 5}$ formed mixtures of the acetate salt, the formate salt and the free amine. The acetate salt forms during the glycosylation reaction which takes place in a $\mathrm{NaOAc} / \mathrm{AcOH}$ buffer (2 M, $\mathrm{pH} 4.5$ ), while the formate salt forms during purification by direct loading of the reaction mixture onto a Bio-Gel P-2 column for size exclusion chromatography using a $0.1 \mathrm{M}$ ammonium formate solution as the mobile phase. The presence of the acetate and formate salts was confirmed by NMR spectroscopy. It was observed that the free amine and/or the salts of the glycoconjugates $\mathbf{2 4}$ and $\mathbf{2 5}$ were altering the observed $\mathrm{pH}$ of the phosphate buffers in which the hydrolysis reaction was to take place, in an unpredictable manner. To mitigate this problem, a biotin probe was installed on each glycoconjugate to mask the free amine and form biotinylated glycoconjugates 26 and $\mathbf{2 7}$. The biotinylation of the "Type B" glycoconjugate to form $\mathbf{2 7}$ was performed by S. Munneke ( $84 \%$ yield, Scheme 21$).{ }^{89}$ The biotinylation of the "Type A" glycoconjugate was achieved by reacting 24 with biotin and the coupling reagent HBTU to form 26 in $80 \%$ yield (Scheme 21). Biotin was chosen to mask the free amine because the chemical shift of its proton resonances was unlikely to overlap with the resonances of interest for the study. Biotin is also a common probe that is often installed on bifunctional linkers for use in biological applications. ${ }^{21}$ The biotinylated conjugates (26 and 27) were then subjected to a variety of $\mathrm{pH}$ values in a phosphate buffer in $\mathrm{D}_{2} \mathrm{O}$. Unfortunately, as the reaction proceeded, a small amount of precipitate formed and coated the inside of the NMR tubes. The identity of the precipitate was not determined as there was too little material to properly isolate from the reaction mixture. It is highly likely that the precipitate was either the glycoconjugate or one of the degradation products (biotinylated free linker or GlcNAc). The presence of the precipitate meant that integrating proton resonances which belong to either the glycoconjugate and/or degradation products would not accurately determine the proportion of either present, as the insoluble precipitate would not register on the ${ }^{1} \mathrm{H}$ NMR spectrum. It is possible that the precipitate could also affect the equilibrium of the hydrolysis reaction, and thus the observed rate of hydrolysis. For example, if the precipitate is one or both degradation products, it lowers the concentration of dissolved degradation products, thereby shifting 
the equilibrium to favour hydrolysis as the reaction proceeds. Additionally, the $O$-methyl peak used as the "internal standard" for the "Type B" conjugate was too close in chemical shift to the glycan signals, as determined by S. Munneke. ${ }^{89}$ Thus, a truly accurate integration was not possible, and ideally the addition of a separate internal standard was needed. However, it was decided that HPLC (high performance liquid chromatography) would be superior to ${ }^{1} \mathrm{H}$ NMR spectroscopy in terms of accuracy and reproducibility of the integrations of each peak, and thus this method was investigated.

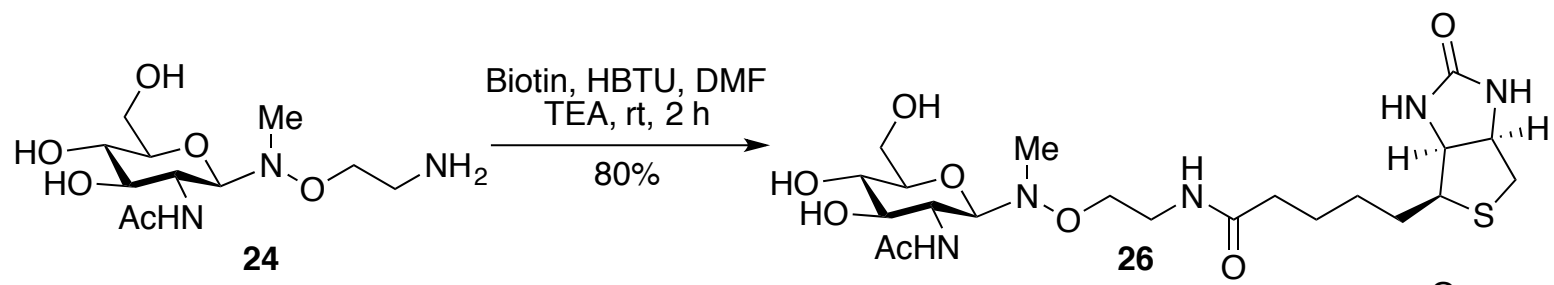

24

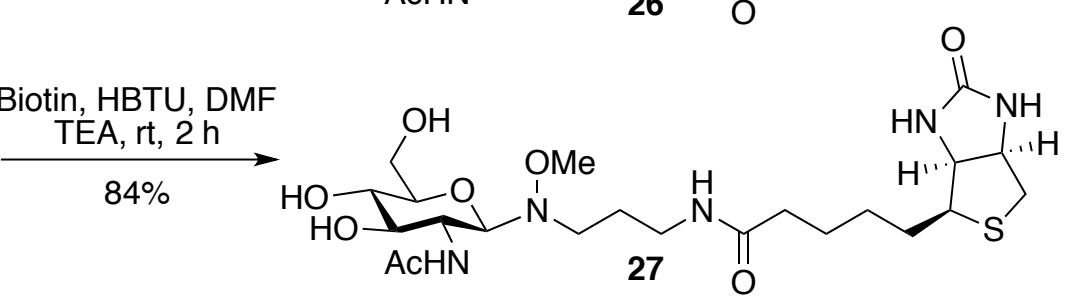

Scheme 21. Conjugation of biotin to glycoconjugates 24 and 25.

\subsubsection{Measuring Rate of Hydrolysis: Design Using HPLC}

In order to use HPLC to measure the extent of hydrolysis of "Type A" and "Type B" oxyamine glycoconjugates, the compounds need to be detected by UV/Vis spectroscopy and therefore need to contain a chromophore. This was achieved by reacting the free amines of glycoconjugates $\mathbf{2 4}$ and $\mathbf{2 5}$ with dansyl chloride to produce the fluorescent glycosides $\mathbf{2 8}$ and $\mathbf{2 9}$ in 70\% and 79\% yield, respectively (Scheme 22). The dansylation of the "Type B" glycoconjugate was performed by S. Munneke. Dansyl is a fluorophore that strongly absorbs at approximately $337 \mathrm{~nm},{ }^{90}$ and was chosen for its water solubility and small size, which means its bulk is unlikely to affect the dynamics of the hydrolysis reaction. A separate internal standard conjugated to the same fluorophore $(\mathbf{3 0}$, Scheme 23) was also synthesised by S. Munneke to accurately determine the quantity of starting material and products present. 

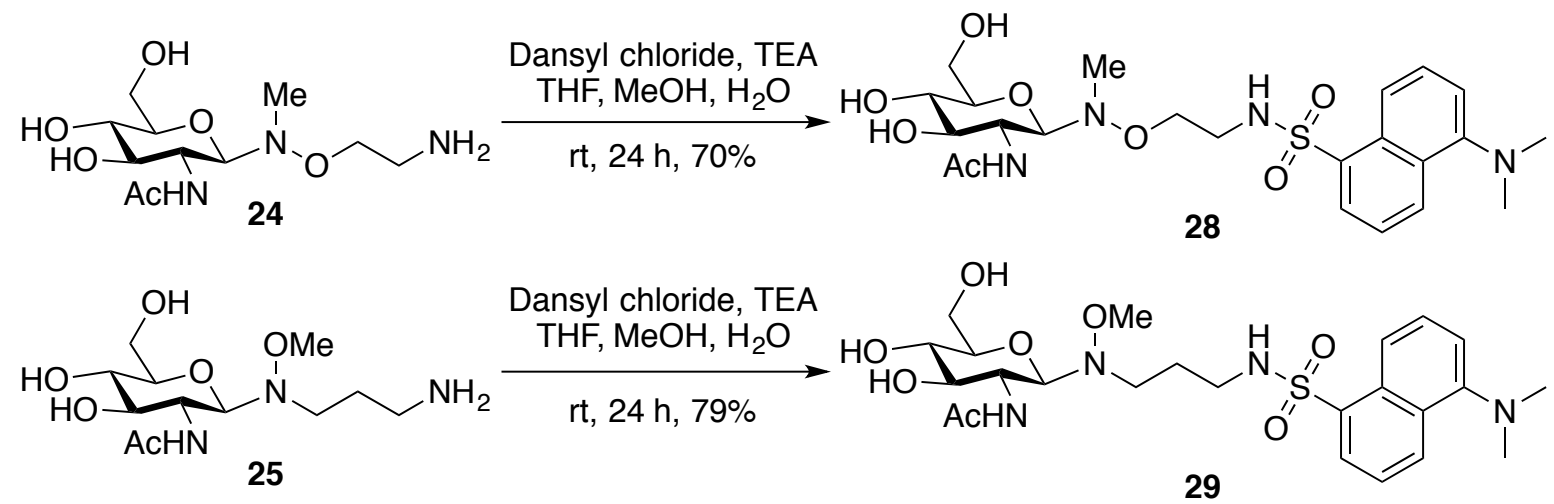

Scheme 22. Reaction of glycoconjugates 24 and 25 with dansyl chloride to give fluorescent glycoconjugates $\mathbf{2 8}$ and 29.

The three fluorescent compounds in each reaction mixture were then detected by UV/Vis spectroscopy during hydrolysis (glycoconjugate 28, free linker $\mathbf{3 1}$ and internal standard 30, Scheme 23) after eluting from a reverse phase column (column: BEH300 $\mathrm{C}_{18} 5 \mu \mathrm{m}$, $4.0 \times 150 \mathrm{~mm}, 35 \% \mathrm{MeCN}$ in $\mathrm{H}_{2} \mathrm{O}$ ). After being measured using $\mathrm{UV} / \mathrm{Vis}$ spectroscopy, the resulting plot (retention time on column $v$ s. absorbance at $337 \mathrm{~nm}$ ) indicated the presence of the 3 separate fluorophores with retention times of 88, 147 and 208 seconds, the identity of which was confirmed by mass spectrometry as glycoconjugate, internal standard and free linker respectively. The integration of the glycoconjugate signal and/or the free linker signal on this plot was measured against the integration of the internal standard to determine the amount of glycoconjugate remaining as a percentage of the original quantity before hydrolysis. Subsequent measurements of the same reaction mixture over time provided the rate of glycoconjugate loss. 

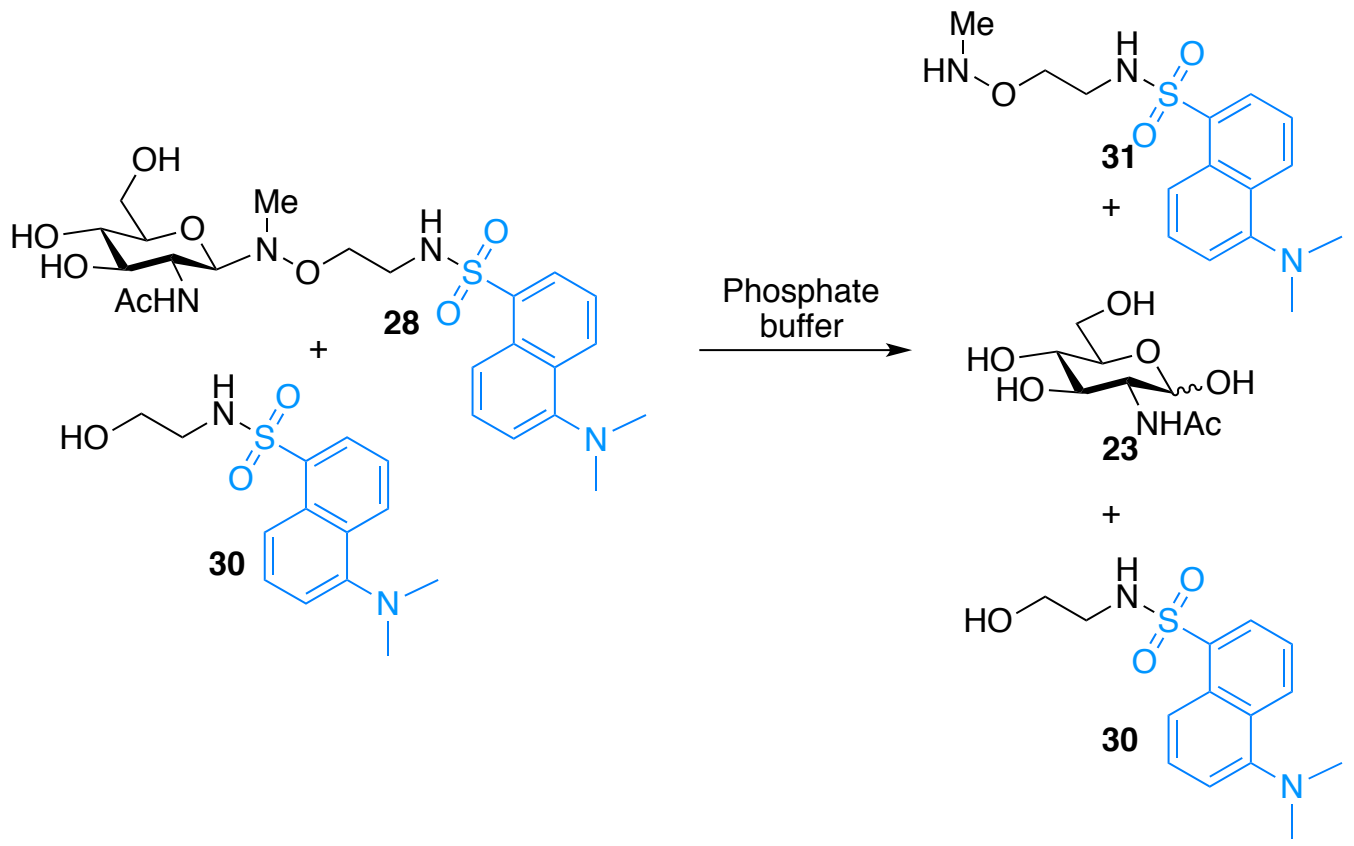

Scheme 23. Hydrolysis of the dansylated "Type A" glycoconjugate (28) forming free linker (31) and GlcNAc (23) products, with dansylated ethanolamine (30) as internal standard. The dansyl functional group is depicted in blue.

\subsubsection{Experimental Variables}

To undertake the hydrolysis experiments, a citric acid- $\mathrm{Na}_{2} \mathrm{HPO}_{4}$ buffer was chosen because it allows for a wide range of $\mathrm{pH}$ values without the need for changing buffer type. To this end, $5 \mathrm{mM}$ solutions of glycosides $\mathbf{2 8}$ or $\mathbf{2 9}$ were prepared using a $100 \mathrm{mM}$ citric acid- $\mathrm{Na}_{2} \mathrm{HPO}_{4}$ buffer at $\mathrm{pH}=3,4,5$ or 6 . The stability of the glycoconjugates in these buffers was then monitored over a course of 30 days. The effect of glycoconjugate concentration on hydrolysis rate was also studied using solutions of each glycoconjugate (28 and 29) at different glycoconjugate concentrations $(2.5,5.0$ or $10.0 \mathrm{mM})$ in $100 \mathrm{mM}$ citric acid- $\mathrm{Na}_{2} \mathrm{HPO}_{4}$ buffer at $\mathrm{pH}$. The effect of buffer strength on glycoside stability was examined, by preparing different solutions of 25, 50, 100 and $200 \mathrm{mM}$ citric acid$\mathrm{Na}_{2} \mathrm{HPO}_{4}$ buffers for each glycoconjugate (28 or 29) at a $\mathrm{pH}$ of 4 and a glycoconjugate concentration of $5 \mathrm{mM}$. Finally, as there is much interest in the biological applications of glycoconjugates synthesised using oxyamine linkers, the rate of hydrolysis of the linkers under biologically relevant conditions were explored. Biological conditions often involve very dilute glycoconjugate concentrations and a $\mathrm{pH}$ of about 7.4. To ensure that the glycoconjugates synthesised in this study are stable under these conditions, a further 
experiment was performed at pH 7.4 in PBS (phosphate-buffered saline) with very dilute glycoconjugate concentrations $(12.5,25,50$ or $100 \mu \mathrm{M})$ for each glycoconjugate type.

During the initial hydrolysis studies, it was also observed that microbial growth started to occur. Accordingly, $\mathrm{NaN}_{3}$ was added to each sample in the experiments to control microbial growth. Through a separate control experiment, it was determined that $\mathrm{NaN}_{3}$ had no effect on the rate of hydrolysis. Through a similar control experiment, it was also shown that the internal standard (30, Scheme 23) added to each sample had no effect on the hydrolysis rate.

\subsubsection{Results: Hydrolysis Studies using HPLC}

The stability of each glycoside was monitored over the course of 30 days in various $\mathrm{pH}$ conditions, glycoside concentrations and buffer strengths. To determine the hydrolysis rates, the half-life for each reaction needed to be calculated. It was previously determined that the hydrolysis of both types of oxyamine linkers proceeds via a pseudo first-order process. $^{38,53}$ Accordingly, the half-lives for the reactions were calculated using the integrated first order rate law written in the form of the exponential decay equation:

$$
A_{t}=A_{0} e^{-k t}
$$

$A_{t}=$ the quantity of starting material at time $\mathrm{t}$,

$A_{0}=$ the quantity of starting material at time $=0$,

$k=$ the decay constant,

$t=$ time.

However, the hydrolysis does not reach completion, but instead reaches a point of equilibrium where the relative quantities of starting material and degradation products remain unchanged. Thus, the above equation is modified to include the quantity of starting material at equilibrium $\left(A_{e q}\right)$ :

$$
A_{t}=\left(A_{0}-A_{e q}\right) e^{-k t}+A_{e q}
$$

$A_{e q}=$ the quantity of starting material at equilibrium. 
The data obtained from each experiment is plotted as a percentage of starting material remaining $v s$. time and fitted to the above equation using the least squares method. This allows for $k$ and $A_{e q}$ to be solved, enabling the half-life $\left(t_{1 / 2}\right)$ to be calculated by solving the equation for $A_{t}=50$.

For the time course experiments assessing the effect of $\mathrm{pH}$ on hydrolysis rates of the two oxyamine linkers, it was found that both glycosides hydrolyse significantly faster as $\mathrm{pH}$ decreases, with negligible degradation at $\mathrm{pH} 6$ (Figure 16). The difference in hydrolysis rate between the two glycosides is minor, though the "Type B" glycoside is slightly more stable with $t_{1 / 2}=1.3 \mathrm{~d}$ at $\mathrm{pH} 3, t_{1 / 2}=7.6 \mathrm{~d}$ at $\mathrm{pH} 4$ and $t_{1 / 2}=73 \mathrm{~d}$ at $\mathrm{pH} 5$ compared to the “Type A" glycoside at $t_{1 / 2}=1.2 \mathrm{~d}$ at $\mathrm{pH} 3, t_{1 / 2}=7.0 \mathrm{~d}$ at $\mathrm{pH} 4$ and $t_{1 / 2}=68 \mathrm{~d}$ at $\mathrm{pH} 5$. However, the final equilibrium concentrations for the "Type A" glycoside is generally slightly higher than those for the "Type B" in each experiment. This means that, while the "Type B" glycoside may be more kinetically stable, the "Type A" is slightly more thermodynamically stable.

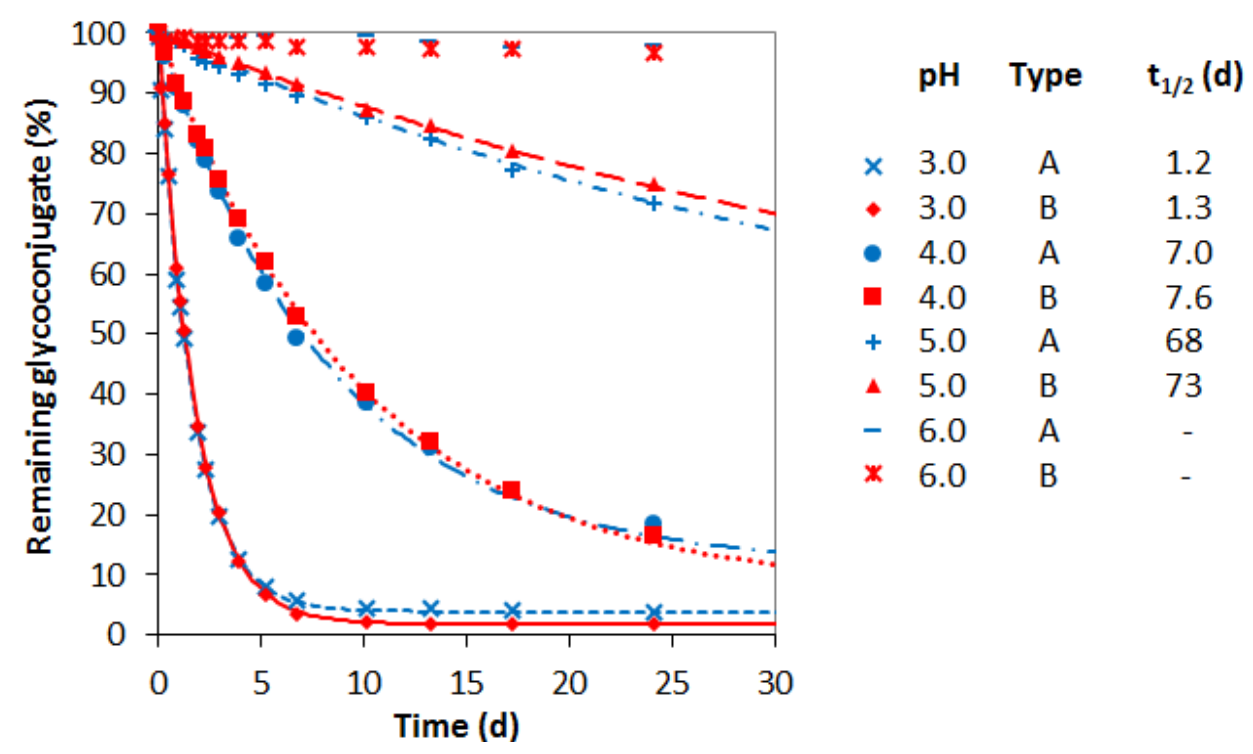

Figure 16. Effect of varying $p H$ on the hydrolysis rates of glycoconjugates 28 and 29. Glycoconjugate concentration $=5 \mathrm{mM}$, buffer strength $=100 \mathrm{mM}$.

When the glycoside concentration was varied ( $\mathrm{pH}=4,100 \mathrm{mM}$ buffer strength), it was found that the more dilute solutions hydrolysed more quickly compared to their more concentrated counterparts (Figure 17). This is not surprising, because it is known that 
very concentrated conditions are required for the formation of these glycosides. ${ }^{38}$ This means that dilute conditions favour the equilibrium towards the free glycan and linker rather than the conjugated glycoside. Again, the "Type B" glycoconjugate was found to be slightly more kinetically stable (as determined by $t_{1 / 2}$ ) compared to the "Type A" derivative, however the "Type A" reached equilibrium at a higher glycoconjugate concentration, as was observed upon changing the $\mathrm{pH}$.

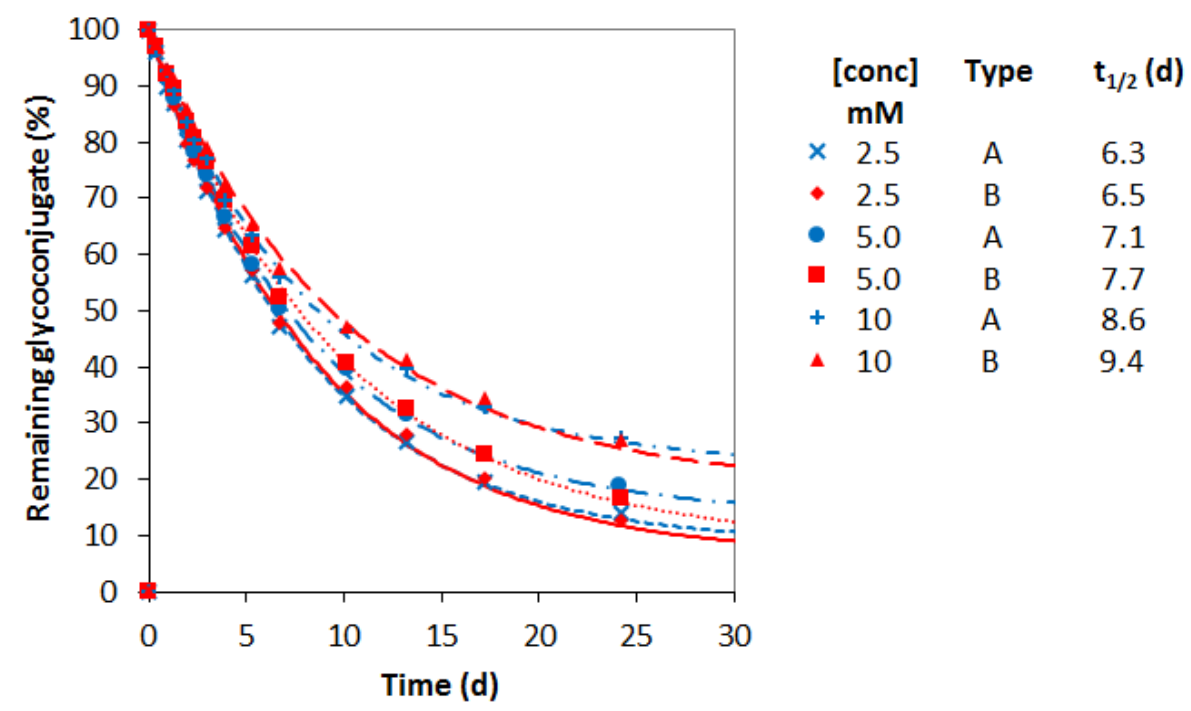

Figure 17. Effect of varying glycoconjugate concentration on the hydrolysis rates of glycoconjugates 28 and 29. Buffer strength $=100 \mathrm{mM}, \mathrm{pH}=4$.

During the investigation of buffer strength on the rate of hydrolysis $(\mathrm{pH}=4,5 \mathrm{mM}$ glycoside concentration) (Figure 18) it was observed that with increasing buffer strength, the rate of hydrolysis also increased. The buffer ions must be catalysing the hydrolysis reaction, thus, by increasing the buffer ion concentration, the reaction is more effectively catalysed. Once again, the "Type B" glycoconjugate had a higher $t_{1 / 2}$ at each conjugate concentration relative to the "Type A", while the "Type A" had a more favourable glycoconjugate:hydrolysed product ratio at equilibrium. 


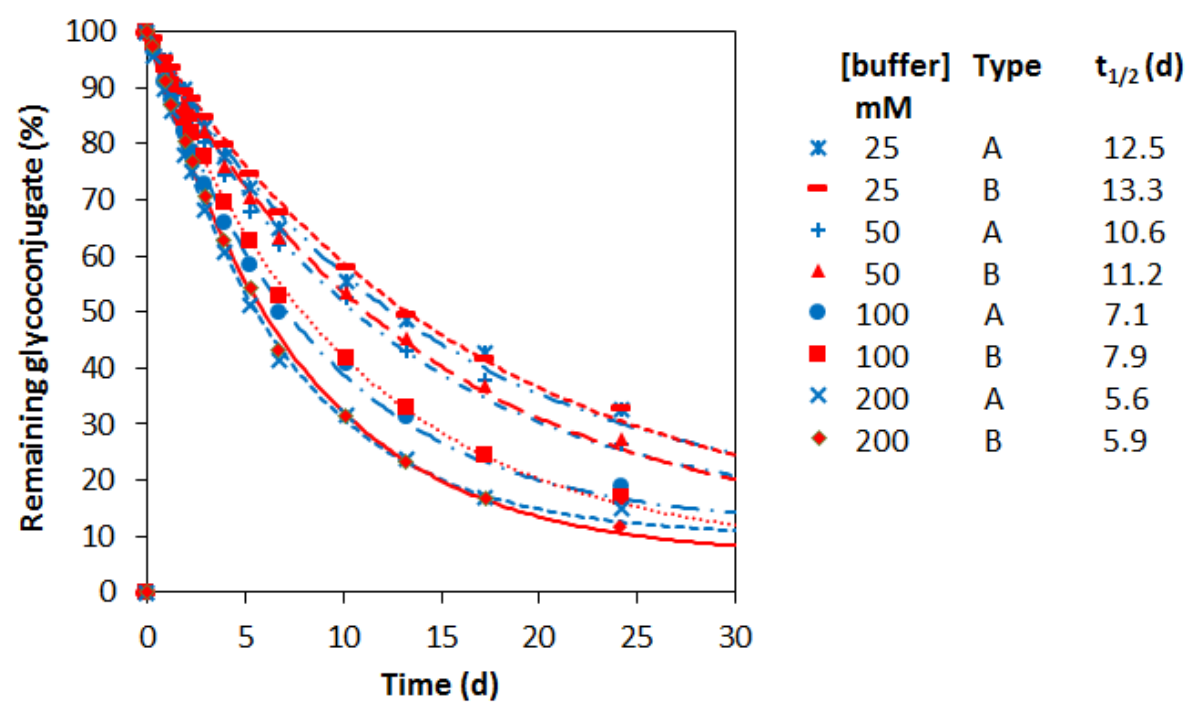

Figure 18. Effect of varying buffer concentration on the rate of hydrolysis of glycoconjugates 28 and 29. Glycoconjugate concentration $=5 \mathrm{mM}, \mathrm{pH}=4$.

A further experiment was conducted to demonstrate the stability of each glycoconjugate type in buffer systems commonly used for biological applications. Both "Type A" and "Type B" glycoconjugates were added PBS ( $\mathrm{pH} 7.4$ ) at very dilute glycoconjugate concentrations $(12.5,25,50$ or $100 \mu \mathrm{M})$. After monitoring these samples over a 35-day period, no hydrolysis products were observed, regardless of glycoconjugate concentration (Figure 19).

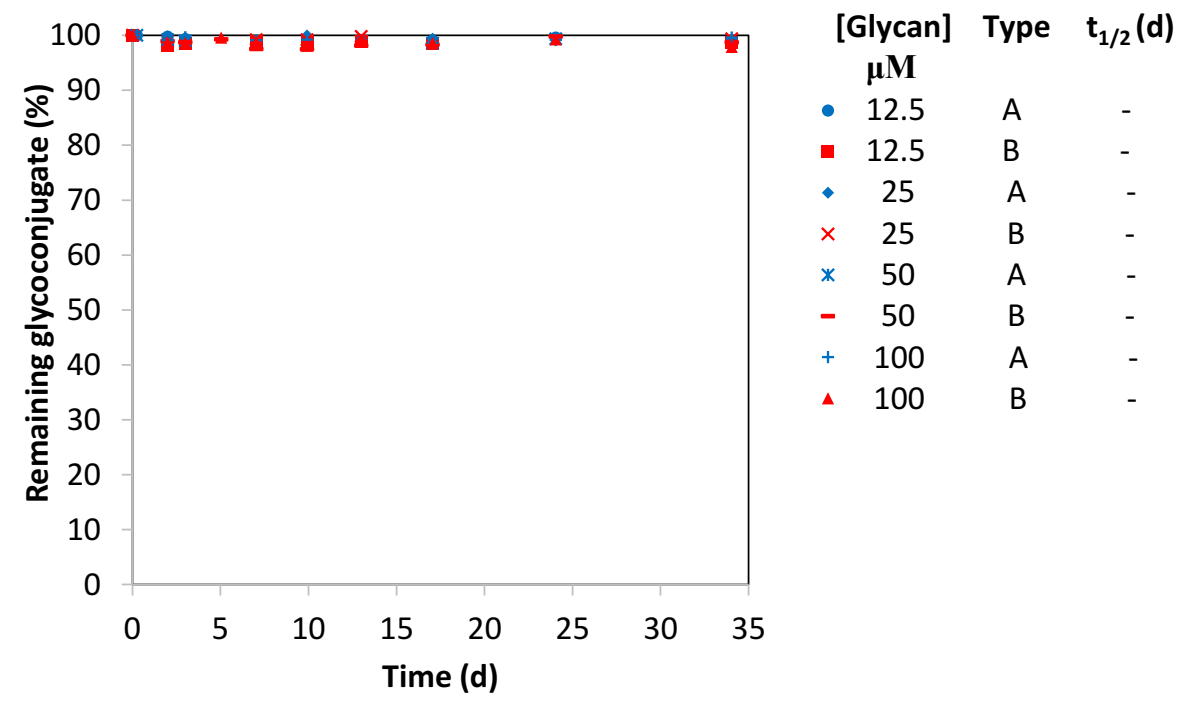

Figure 19. Hydrolysis studies in PBS with varying glycoconjugate concentration. 
Our "Type A" and "Type B" glycosides harbour a similar level of stability towards hydrolysis compared to other structurally similar "Type A" glycosyloxyamines described in the literature. ${ }^{43,50,66}$ These studies have only examined the effect that varying $\mathrm{pH}$ has on the hydrolytic stability of "Type A" glycosyloxyamines, each study also varies in buffer and glycoside concentration. The results of this chapter have indicated that varying buffer and glycoside concentration has a significant effect on the rate of hydrolysis. However, a tentative comparison can be conducted bearing these variables in mind. A comparison can also only be made with glycosyloxyamines which have a similar structure to our glycosides as work done by Nitz and co-workers have demonstrated that different substituents and/or saccharides have a profound effect on the stability of glycosyloxyamines. ${ }^{43}$ Cló et al. provided half-lives of $28 \mathrm{~h}$ and $34 \mathrm{~h}$ for glycosides 32 and 33 (Figure 20A) respectively at $\mathrm{pH} 4$ with a buffer concentration of $50 \mathrm{mM}$ and a glycoside concentration of $1 \mathrm{mM} .{ }^{66}$ Nitz and co-workers afforded half-lives of $76 \mathrm{~h}$ and $350 \mathrm{~h}$ for glycosides 34 and 35 (Figure 20B) respectively at $\mathrm{pH} 4$ in a $20 \mathrm{mM}$ buffer and with a glycoside concentration of $2 \mathrm{mM}^{43}$ Finally, Langenhan et al. obtained half-lives varying from $9.1 \mathrm{~h}$ to $31.8 \mathrm{~h}$ for glucosides at $\mathrm{pH} 3$ with a $20 \mathrm{mM}$ buffer concentration and a $2 \mathrm{mM}$ glycoside concentration. ${ }^{50}$ Our glycosides have half-lives of $168 \mathrm{~h}$ and $182 \mathrm{~h}$ for $\mathbf{2 8}$ and 29 respectively, and are on the more stable end of the scale obtained from the literature $(9.1 \mathrm{~h}-350 \mathrm{~h})$. This is likely to be due to the different monosaccharides present in the glycosides (GlcNAc vs. Glc) and/or the different glycoside and buffer concentrations between experiments.

A<smiles>[R16]CCON(C)C(O)C(O)C(O)C(O)CO</smiles>

32

B<smiles>CC1NC(N(C)OCc2ccccc2)C(C)(O)C(O)C1O</smiles>
34<smiles>CN(OCc1ccc(C(=O)NCCN)cc1)C(O)C(O)C(CO)CO</smiles>

33

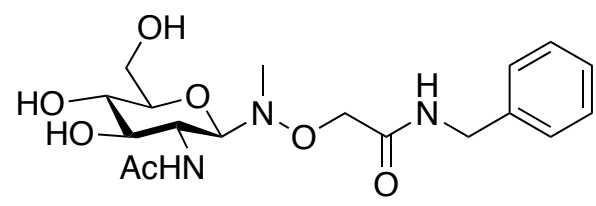

35

Figure 20. A) Glycosyloxyamines 32 and 33 synthesised and tested by Cló et al. B) Glycosyloxyamines 34 and 35 synthesised and tested by Nitz and co-workers. 


\subsection{Conclusion}

In this chapter, the ease of synthesis and hydrolytic stability of glycosides containing similar "Type A" and "Type B" oxyamine linkers were explored. The results of this study have shown that, overall, there is little difference between the hydrolysis rates of "Type A" and "Type B" glycosides.

The synthesis of the "Type A" linker has now been improved from an overall yield of $8 \%$ over 6 steps $^{51}$ to an overall yield of $38 \%$ over 4 steps. While this new synthetic route is more rapid and higher yielding than Bohorov's original route, the "Type B" linker still has a more concise and adaptable synthetic route. The same 3-step-1-pot synthetic procedure developed by Munneke et al. can be adapted to produce "Type B" linkers with different terminal functional groups, producing overall yields ranging from $51 \%$ to 96\%. ${ }^{53}$ Both the "Type A" and "Type B" linkers, 4 and 22 can also be conjugated to representative glycans and probes such as biotin or dansyl in similar yields. The effect of $\mathrm{pH}$, glycoside concentration and buffer strength were examined regarding the hydrolytic stability of the "Type A" and "Type B" glycosides. The "Type B" glycosides were found to have marginally greater kinetic stability, while the "Type A" glycosides were slightly more thermodynamically stable across all the variables tested. However, the modest differences in hydrolysis rates between the two types are likely to be minimal when used in practice. Overall, it was found that both "Type A" and "Type B" linkers are excellent candidates for the synthesis of glycoconjugates. 


\section{Chapter 3}

\section{$\mathrm{p} K_{\mathrm{a}}$ Determination of the Conjugate Acid of Glycosyloxyamines}

\subsection{Introduction}

The acid dissociation constant $\left(K_{\mathrm{a}}\right)$ is a measure of how readily an acid dissociates into a proton (in the form of protonated solvent) and its conjugate base. ${ }^{87}$ A negative log scale provides a convenient means to compare $K_{\mathrm{a}}$ values, as defined by the $\mathrm{p} K_{\mathrm{a}}$ (equation 3 ). Thus, the lower the $\mathrm{p} K_{\mathrm{a}}$, the stronger the acid.

$$
\mathrm{p} K_{\mathrm{a}}=-\log _{10} K_{\mathrm{a}}
$$

Measuring the $\mathrm{p} K_{\mathrm{a}}$ values of specific functional groups within a molecule provides insight into how the acidity of the molecule can be modified by different substituents. ${ }^{91}$ These $\mathrm{p} K_{\mathrm{a}}$ values can therefore tell us the extent of the electron withdrawing or donating character of the different substituents. For example, the $\mathrm{p} K_{\mathrm{a}}$ of acetic acid in $\mathrm{H}_{2} \mathrm{O}$ is $4.76,{ }^{91}$ while the $\mathrm{p} K_{\mathrm{a}}$ of trifluoroacetic acid (TFA) is $-0.30 .^{92}$ The acidity of the carboxylic acid has increased significantly by replacing the methyl substituent with a trifluoromethyl substituent. The methyl substituent is slightly electron donating while the trifluoromethyl substituent is significantly electron withdrawing due to the inductive effect of the electronegative fluorine atoms. Consequently, the negative charge of the carboxyl anion of TFA is better stabilised, making proton dissociation more favourable. ${ }^{87}$

The $\mathrm{p} K_{\mathrm{a}}$ of specific positions within a molecule can also give a measure of the molecule's stability if protonation or deprotonation of that site is required for degradation. Timell et al. compared the relative rates of hydrolysis under acidic conditions of methyl glycopyranosides which differ in the substituent present on the C-5 position. ${ }^{93}$ It was found that methyl $\alpha$-D-glucopyranoside (11) was more stable than methyl $\alpha$-Dxylopyranoside (36) (Figure 21). This observation correlates with the electron affinity of the substituent on the C-5 position. The C-5 position of the xylopyranoside is more electron rich than that of the glucopyranoside. This allows the anomeric oxygen of the xylopyranoside to be more readily protonated than the glucopyranoside. The 
oxocarbenium ion is then formed more quickly, producing a faster rate of hydrolysis (Scheme 24). ${ }^{93}$ Thus, the conjugate acid of the xylopyranoside is a weaker acid (higher $\mathrm{p} K_{\mathrm{a}}$ ) than the conjugate acid of the glucopyranoside and is therefore more likely to be protonated. The electron rich xylopyranoside is also better able to stabilise the oxocarbenium ion once formed.
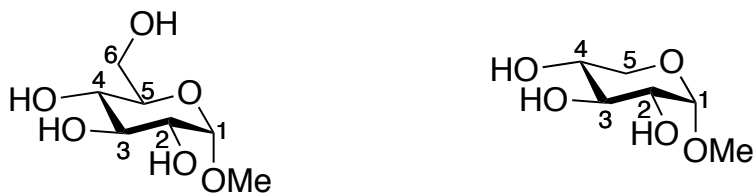

methyl $\alpha$-D-glucopyranoside (11) methyl $\alpha$-D-xylopyranoside (36)

Figure 21. Structures for methyl $\alpha$-D-glucopyranoside (11) and methyl $\alpha-D-$ xylopyranoside (36).

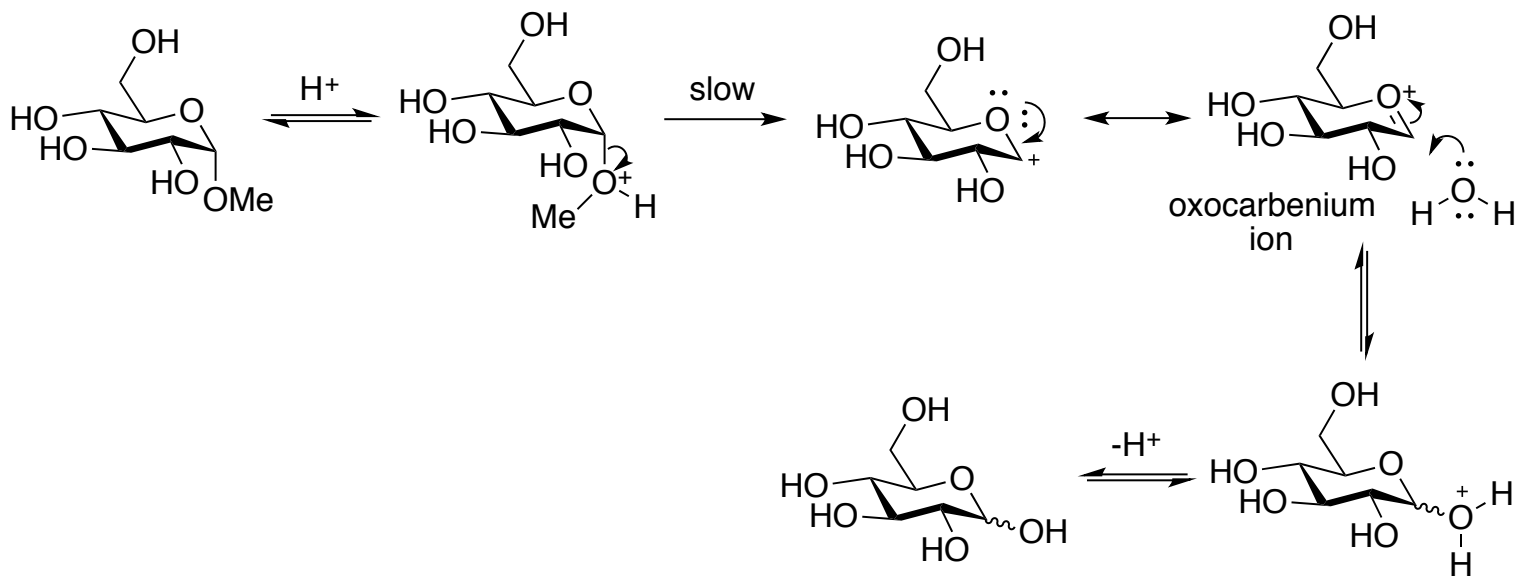

Scheme 24. Timell's proposed mechanism for the hydrolysis of methyl pyranosides under acidic conditions. ${ }^{93}$

\subsubsection{The Hydrolysis of N-glycosyloxyamines}

There is much interest in understanding why $N$-glycosyloxyamines are stable under basic, neutral and mildly acidic conditions. As discussed in Chapter two, previous studies conducted on the hydrolytic stability of glycosyloxyamines, including work undertaken as part of this thesis, have demonstrated that the rate of hydrolysis of $\mathrm{N}$ glycosyloxyamines is highly $\mathrm{pH}$ dependent. ${ }^{38,43,50,53,66} \mathrm{As}$ the $\mathrm{pH}$ is lowered, the rate of hydrolysis of glycosyloxyamines increases, indicating that some form of acid catalysis aids in the hydrolysis of the glycosidic bond. Studies also demonstrated that more electron rich monosaccharides and/or $N$-alkyl substituents increase the rate of hydrolysis, ${ }^{43}, 66$ 
which demonstrates that protonation is crucial for degradation to occur and that this protonation step must occur before or at the rate-determining step (RDS). ${ }^{43}$

The putative mechanism for the hydrolysis of glycosyloxyamines involves the formation of an oxyiminium ion, following ring oxygen protonation, (Scheme 25) (endocyclic C-O bond cleavage) rather than initial protonation of the anomeric nitrogen and the formation of an oxocarbenium ion (exocyclic C-N bond cleavage). ${ }^{43,94}$ This is due to the principle of microscopic reversibility, ${ }^{87}$ the mechanism of hydrolysis of glycosyloxyamines must proceed via the same pathway as their formation, which will be the pathway with the lowest energy. A mechanism of hydrolysis involving the protonation of the anomeric nitrogen, followed by oxocarbenium ion formation and subsequent attack of water must, therefore, be disregarded. Instead, the mechanism must follow a route where water attacks at the iminium ion.

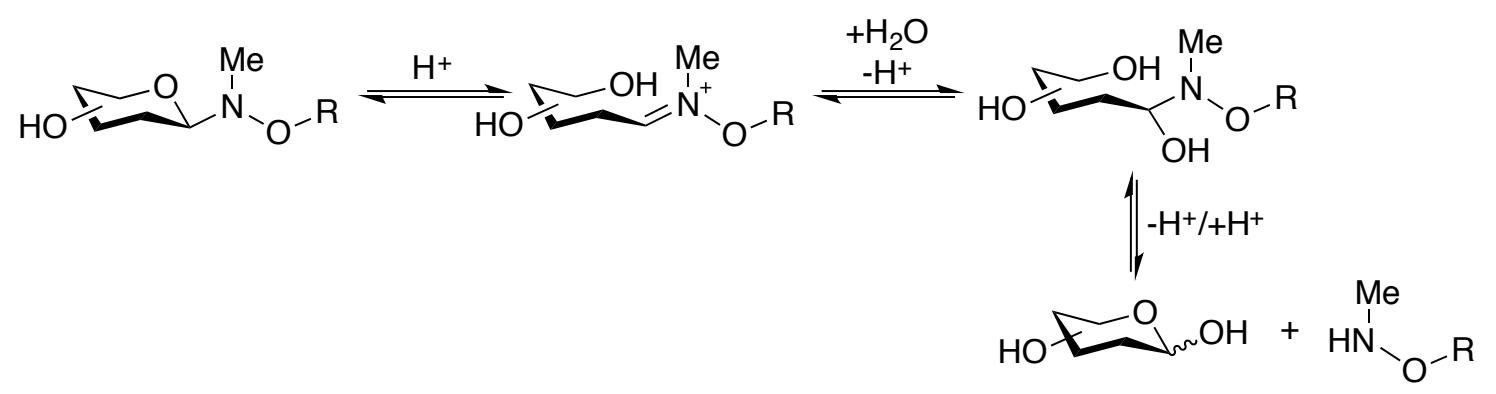

Scheme 25. Accepted steps for the mechanism of the hydrolysis of glycosyloxyamines. ${ }^{43}$

If the $\mathrm{p} K_{\mathrm{a}}$ of the conjugate acid of the glycosyloxyamine was determined, more information could be obtained about its high stability under neutral and basic conditions. The $\mathrm{p} K_{\mathrm{a}}$ can also provide information on the optimal $\mathrm{pH}$ at which the hydrolysis will take place. By finding the $\mathrm{p} K_{\mathrm{a}}$ of the conjugate acid of the most basic position of the glycosyloxyamine, which should be the (protonated) anomeric nitrogen (Figure 22), it can be inferred that the $\mathrm{p} K_{\mathrm{a}}$ of the protonated ring oxygen (and all other protonated hydroxyls) will be lower. This is important because it is the ring oxygen which ultimately needs to become protonated for hydrolysis to occur. ${ }^{94}$ The $\mathrm{p} K_{\mathrm{a}} \mathrm{s}$ of the conjugate acid of glycosyloxyamines have not yet been experimentally determined. This will be the focus of this chapter, as determining the $\mathrm{p} K_{\mathrm{a}} \mathrm{s}$ of the conjugate acid of glycosyloxyamines will not only provide more information on the stability of these conjugates, but will also give insight into the type of acid catalysis that occurs during the hydrolysis reaction. 


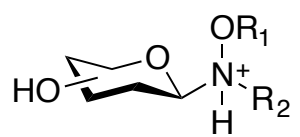

Figure 22. The structure for the conjugate acid of a generic glycosyloxyamine.

\subsubsection{Acid and Base Catalysis}

Acids and bases offer a common way of increasing the rate of a reaction. ${ }^{87}$ There are two types of acid and base catalysts: specific acid and general acid catalysts, and specific base and general base catalysts. Specific catalysts are responsible for the $\mathrm{pH}$ of the solution $\left(\mathrm{H}_{3} \mathrm{O}^{+}\right.$and $\left.\mathrm{OH}^{-}\right)$and are the simplest Brønsted species. They allow for the complete protonation/deprotonation of the substrate to occur before the reaction commences. General catalysts, denoted HA and A; , aid in protonation/deprotonation during the RDS and include all general Brønsted acids or bases in solution, including undissociated acids. $^{87,95}$

\subsubsection{Specific Acid Catalysis}

Specific acid catalysis (SAC) involves the fast and complete protonation of the electrophile, to make it more electrophilic, followed by the slow rate-determining step, which has been accelerated in relation to the uncatalysed reaction. The hydronium ion $\left(\mathrm{H}_{3} \mathrm{O}^{+}\right)$is the only Brønsted acid strong enough to protonate the substrate without aid from any other processes. ${ }^{87}$ The rate is thus specifically dependent on the concentration of $\mathrm{H}_{3} \mathrm{O}^{+}$and therefore $\mathrm{pH}$, with the concentration of other buffers in solution having no effect on the rate of the reaction. ${ }^{96}$ For SAC to be effective however, the $\mathrm{pH}$ of the solution needs to be equal to, or lower than the $\mathrm{p} K_{\mathrm{a}}$ of the conjugate acid of the substrate. A $\mathrm{pH}$ significantly higher than the $\mathrm{p} K_{\mathrm{a}}$ will not yield enough protonated substrate for catalysis to occur. An inverse solvent isotope effect $\left(k\left[\mathrm{D}_{2} \mathrm{O}\right]>k\left[\mathrm{H}_{2} \mathrm{O}\right]\right)$ is also indicative of SAC. This is because $\mathrm{H}_{2} \mathrm{O}$ is better at solvating $\mathrm{H}_{3} \mathrm{O}^{+}$than $\mathrm{D}_{2} \mathrm{O}$ is at solvating $\mathrm{D}_{3} \mathrm{O}^{+}$(due to the longer $\mathrm{O}-\mathrm{H}$ bond $v s$. O-D bond). ${ }^{87}$ This allows $\mathrm{D}_{3} \mathrm{O}^{+}$to protonate a substrate more easily than $\mathrm{H}_{3} \mathrm{O}^{+}$. Thus, at any one time, a higher percentage of the substrate is protonated in $\mathrm{D}_{2} \mathrm{O}$ than in $\mathrm{H}_{2} \mathrm{O}$, increasing the rate of reaction. ${ }^{87}$ 


\subsubsection{Specific Base Catalysis}

Analogous to SAC is specific base catalysis (SBC), which involves the fast deprotonation of the nucleophile to make it more nucleophilic in a pre-equilibrium step, followed by the rate-determining step. As with SAC, the reaction rate depends entirely on the $\mathrm{pH}$ of the solution as only the $\mathrm{OH}^{-}$ion is a strong enough base for effective catalysis. For SBC to occur, the $\mathrm{pH}$ must be equal to or higher than the $\mathrm{p} K_{\mathrm{a}}$ of the nucleophilic substrate to enable deprotonation. An inverse solvent isotope effect is also observed for SBC. ${ }^{87}$

\subsubsection{General Acid Catalysis}

Acids which are too weak to protonate a substrate can still act as a catalyst during general acid catalysis (GAC) ${ }^{87}$ Therefore, the reaction rate not only depends on the $\mathrm{pH}\left(\mathrm{H}_{3} \mathrm{O}^{+}\right.$ concentration) of the solution, but also on the concentration of other undissociated acids, HA. Because the reaction is being catalysed by weak acids during GAC, the protonation of the substrate cannot occur before the RDS, but instead occurs during the RDS where the attack of the nucleophile aids in protonation of the electrophile by making the electrophile more basic. Similarly, the protonation of the electrophile during the RDS aids nucleophile attack. Thus, GAC often involves a termolecular transition state (TS), which is demonstrated by a large negative value for the entropy of activation $\left(\Delta S^{\ddagger}\right){ }^{87}$ A normal kinetic isotope effect (KIE) $\left(k_{\mathrm{H}}>k_{\mathrm{D}}\right)$ is observed during GAC, because a bond to hydrogen is being formed during the RDS. Reactions involving the heavier deuterium isotope will therefore be slower. ${ }^{87}$

\subsubsection{General Base Catalysis}

General base catalysis (GBC) involves the deprotonation of a substrate during the RDS by a weak base. The rate of reaction is thus dependent on the concentration of all bases in solution as well as $\mathrm{pH}$. As with GAC, a large negative value for the entropy of activation is often observed for GBC and a normal KIE is also observed as deprotonation occurs during the RDS. ${ }^{87}$ 


\subsubsection{Methods for the Determination of $p K_{a}$ Values}

The $\mathrm{p} K_{\mathrm{a}}$ of an organic compound provides essential information about its chemical behaviour. Many methods have been developed to determine the $\mathrm{p} K_{\mathrm{a}}$ of different chemical compounds, each with their own advantages and disadvantages. These methods include simple acid-base titrations, the use of NMR spectroscopy, and high performance liquid chromatography (HPLC), amongst others. ${ }^{97}$

\subsubsection{Acid-Base Titration}

The $\mathrm{p} K_{\mathrm{a}}$ of weak organic acids or bases is commonly determined via titration against a strong base or acid respectively. A titration curve is generated by plotting the $\mathrm{pH}$ of the solution, containing the weak acid/base, against the volume of strong base/acid added. For any acid, the $\mathrm{p} K_{\mathrm{a}}$ is related to $\mathrm{pH}$ via the Henderson-Hasselbalch equation (equation $4):^{87}$

$$
\mathrm{pH}=\mathrm{p} K_{\mathrm{a}}+\log \left(\frac{\left[\mathrm{A}^{`}\right]}{[\mathrm{HA}]}\right)
$$

where $[\mathrm{HA}]=$ the molar concentration of the undissociated acid and $\left[\mathrm{A}^{-}\right]=$the molar concentration of the conjugate base of the acid. Thus, the $\mathrm{pH}=\mathrm{p} K_{\mathrm{a}}$ when the concentration of $\mathrm{HA}$ and $\mathrm{A}^{-}$are equal, which is the half-equivalence point on the titration curve. At the half-equivalence point the $\Delta \mathrm{pH} / \Delta$ volume ratio is small, allowing the $\mathrm{p} K_{\mathrm{a}}$ to be determined with good accuracy. However, this method is only suitable for weak acids or bases and is thus limited to a specific $\mathrm{pH}$ range. ${ }^{98,99}$

\subsubsection{NMR Chemical Shift}

${ }^{1} \mathrm{H}$ or ${ }^{13} \mathrm{C}$ NMR spectroscopy can also be used to determine the $\mathrm{p} K_{\mathrm{a}}$ of a molecule by plotting the change in chemical shift of resonances closest to the site of protonation as a function of $\mathrm{pH} .{ }^{97}$ The proton and/or carbon resonances of the protonated species are expected to have higher chemical shifts due to the reduction of electron density around the nuclei of atoms near the site of protonation. Proton exchange is usually rapid compared to the NMR time-scale, thus producing signals which have an averaged chemical shift of the protonated and unprotonated species. ${ }^{100}$ As the $\mathrm{pH}$ decreases, more of the protonated species are present in solution, thereby increasing the chemical shift of affected resonances. ${ }^{97}$ Plotting the observed chemical shift $v$ s. $\mathrm{pH}$ generates a sigmoidal 
curve where the $\mathrm{p} K_{\mathrm{a}}$ is located at the inflection point. ${ }^{97,101}$ An advantage of this method for the determination of $\mathrm{p} K_{\mathrm{a}}$ is the elimination of the requirement to perform a continuous titration, as known quantities of acid or base can be added to separate samples, which can then be measured. Microscopic $\mathrm{p} K_{\mathrm{a}}$ values of polyprotic acids can also be obtained using this method. ${ }^{97}$

\subsubsection{High Performance Liquid Chromatography}

The retention time of a compound on reverse phase HPLC changes when the $\mathrm{pH}$ is altered. ${ }^{97}$ This is due to the different affinities towards the immobile phase experienced by the protonated species relative to the unprotonated species. Accordingly, models have been developed which relate the retention or capacity factor $(\mathrm{k})$ to the degree of dissociation of the acid $(\alpha)$. A plot of $\mathrm{k} v s$. $\mathrm{pH}$ provides a sigmoidal curve with the $\mathrm{p} K_{\mathrm{a}}$ at the inflection point. This method also works for samples which are not $100 \%$ pure as HPLC is a separation technique. The analyte must, however, contain a chromophore for detection. The retention factor of the unionised species $\left(\mathrm{k}_{0}\right)$ is often too big to be measured, meaning that the full range of $\alpha$ cannot always be utilised, reducing the accuracy of the obtained $\mathrm{p} K_{\mathrm{a}}$ value. ${ }^{97}$

\subsection{Results and Discussion}

The objective of the work in this chapter is to determine and examine the $\mathrm{p} K_{\mathrm{a}}$ of a generic glycosyloxyamine. Using these results in conjunction with the results of the hydrolysis studies discussed in Chapter two, it is anticipated that further insight into the mechanism of hydrolysis and the type of catalysis involved can be obtained.

\subsubsection{Model Glycoconjugate Synthesis}

To determine the $\mathrm{p} K_{\mathrm{a}}$ of glycosyloxyamines, studies using a simple, representative glycoconjugate, $\quad N$-(2-acetamido-2-deoxy- $\beta$-D-glucopyranosyl)- $N$-methyl- $O$-methyl hydroxylamine (37) (Scheme 26) were undertaken. The structure of 37 closely resembles those synthesised for the oxyamine stability studies in Chapter two (4 and 22). However, the use of $\mathrm{N}, \mathrm{O}$-dimethylhydroxylamine as the oxyamine means that it is both a "Type A" 
or a "Type B" glycoconjugate, thereby negating any possible affect that the difference in substitution pattern may have on $\mathrm{p} K_{\mathrm{a}}$.

The synthesis of conjugate 37 was achieved by reacting GlcNAc (23) with $N, O$ dimethylhydroxylamine hydrochloride in an acetate buffer at $\mathrm{pH} 4.5$ (2 M) for 24 hours at room temperature (Scheme 26). Purification by size exclusion chromatography then provided 37 in $65 \%$ yield. To confirm that the $\beta$-pyranose product had been formed, ${ }^{1} \mathrm{H}$ NMR spectra of $\mathbf{3 7}$ were obtained. The anomeric proton resonance exhibited a ${ }^{3} J_{1,2}=$ 9.6 Hz, which is consistent with 1,2-trans-diaxial coupling constants in $\beta$ glucopyranosides. Furthermore, conjugation of $\mathrm{N}, \mathrm{O}$-dimethylhydroxylamine to GlcNAc was confirmed by the presence of an HMBC between the $N$-methyl and anomeric resonances (Figure 23).

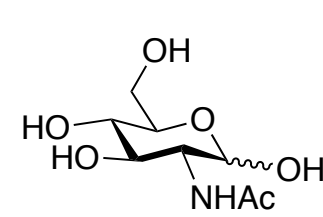

23

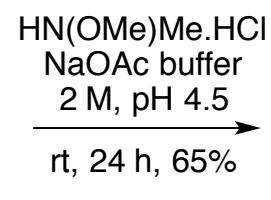

$\stackrel{2 \mathrm{M}, \mathrm{pH} 4.5}{\mathrm{rt}, 24 \mathrm{~h}, 65 \%}$
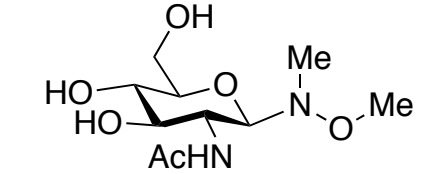

37

Scheme 26. Synthesis of representative glycosyloxyamine 37. 


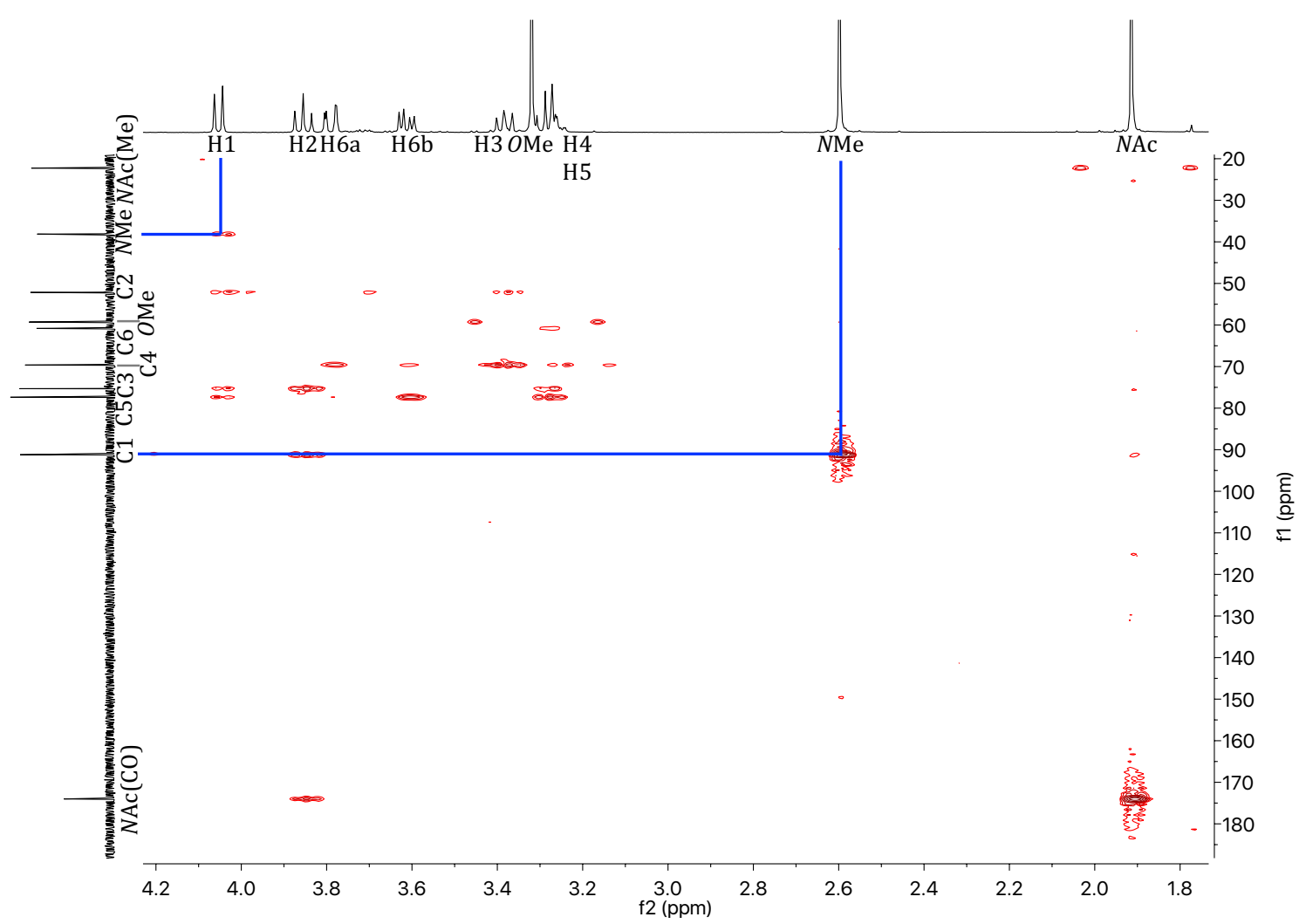

Figure 23. $\mathrm{HMBC}$ for conjugate $37\left(\mathrm{D}_{2} \mathrm{O}, 500 \mathrm{MHz}\right)$.

\subsubsection{Acid-Base Titrations for $p K_{a}$ Determination}

Initially, the acid-base titration method was used in an attempt to determine the $\mathrm{p} K_{\mathrm{a}}$ of the conjugate acid of glycoconjugate 37 . The glycoconjugate was treated as a weak base and titrated against $\mathrm{HCl}$, each at a concentration of $0.01 \mathrm{M}$. However, after plotting the $\mathrm{pH}$ of the solution against volume of $\mathrm{HCl}$ added (Figure 24), no half-equivalence point could be detected. Accordingly, $50 \mu \mathrm{L}$ of $1 \mathrm{M} \mathrm{NaOH}$ was added to the glycoside solution before titrating against $\mathrm{HCl}$ to ensure that none of the glycoside was in its conjugate acid form. The resulting plot more accurately resembled a strong-acid-strong-base titration curve rather than a strong-acid-weak-base curve, however, once again no halfequivalence point could be determined. These results indicated that the half-equivalence point, and therefore the $\mathrm{p} K_{\mathrm{a}}$, was either at a much higher or much lower $\mathrm{pH}$ than what had been tested. An attempt was then made to titrate the glycoside as a weak acid against $\mathrm{NaOH}$ (both at $0.01 \mathrm{M}$ ) to evaluate whether the half-equivalence point was at a higher $\mathrm{pH}$. Again, no half-equivalence point was observed. Unfortunately, a $\mathrm{pH}$ lower than 2 could not be achieved using this method. Using more concentrated $\mathrm{HCl}$ would lead to a large $\Delta \mathrm{pH} / \Delta$ volume, which may mean that the observed half-equivalence point would be 
inaccurate, if indeed it were detectable at all. It was thus decided to change to the NMR titration method for the determination of the $\mathrm{p} K_{\mathrm{a}}$ of the conjugate acid of 37 .

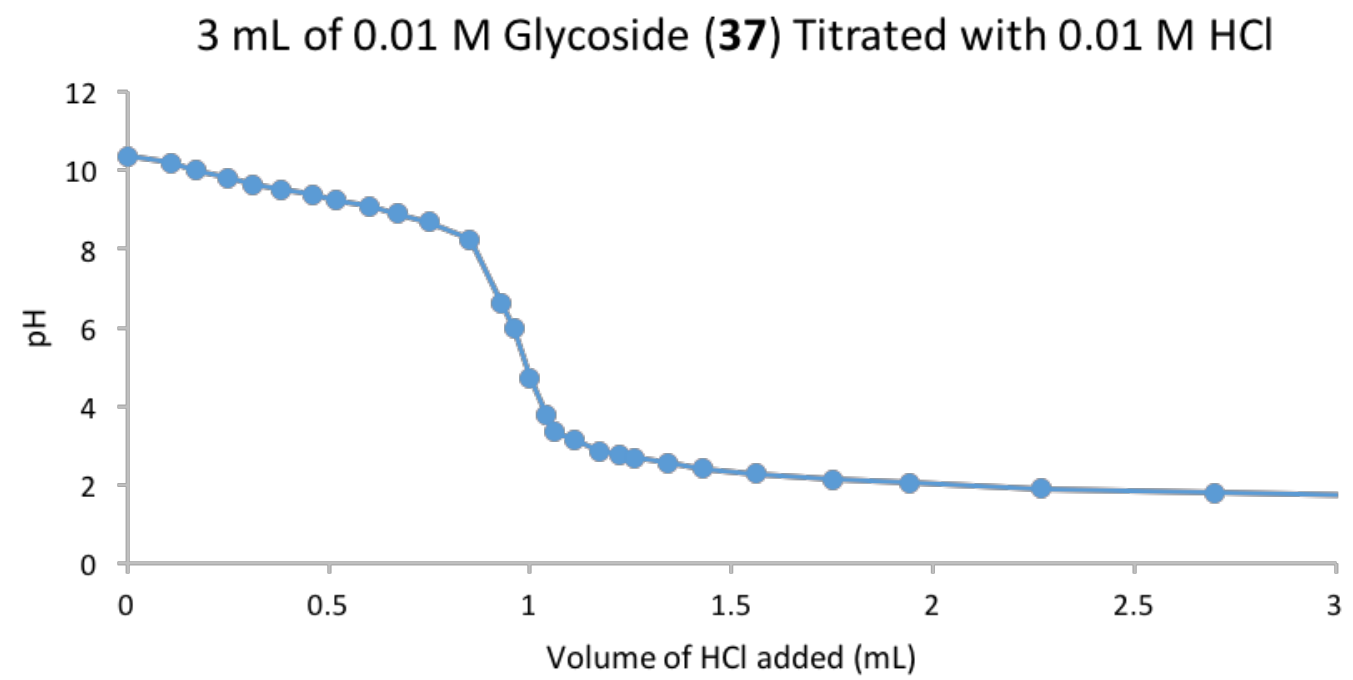

Figure 24. Plot for the titration of $3 \mathrm{~mL} 0.01 \mathrm{M}$ glycoside 37 against $0.01 \mathrm{M} \mathrm{HCl}$, illustrating no buffering by acid or base.

\subsubsection{NMR Titrations for $p K_{a}$ Determination}

The use of NMR spectroscopy for the determination of $\mathrm{p} K_{\mathrm{a}}$ is an accurate technique that can span a wide $\mathrm{pH}$ range. ${ }^{97}$ There is no need for a continuous titration as each $\mathrm{pH}$ titration point is obtained from a separate NMR sample. This means that the $\mathrm{pH}$ does not need to be instantly measured before the titration can continue, but rather, can be measured sometime after the NMR spectrum for that sample has been obtained.

\subsubsection{1 ${ }^{13}$ C NMR Titrations to Determine $p K_{a}$}

The results from the acid-base titrations indicated that the $\mathrm{p} K_{\mathrm{a}}$ of $\mathbf{3 7}$ was either at a very high or low $\mathrm{pH}$, beyond that which could be measured using the acid-base titration methodology. Accordingly, ${ }^{13} \mathrm{C}$ NMR spectroscopy was used to measure any change in chemical shift for 37 at very high pD values (7-14). Each NMR sample contained a 10 $\mathrm{mg} / \mathrm{mL}$ solution of 37 in $\mathrm{D}_{2} \mathrm{O}$, and a varying quantity of $\mathrm{NaOD}$ in $\mathrm{D}_{2} \mathrm{O}$ to obtain six different samples at $\mathrm{pD} 8,9,10,11,12$ and 14 . A sample at $\mathrm{pD} 7$ was also prepared using $\mathrm{D}_{2} \mathrm{O}$ only. However, there was no significant change in chemical shift for any of the resonances of $\mathbf{3 7}$ with increasing $\mathrm{pD}$. Thus, it was concluded that the $\mathrm{p} K_{\mathrm{a}}$ of $\mathbf{3 7}$ must 
reside at a very low, rather than very high, $\mathrm{pH} .{ }^{13} \mathrm{C}$ NMR spectroscopy was not suitable for these low pD measurements as the glycoside is unstable and will degrade during the time required to obtain a ${ }^{13} \mathrm{C}$ NMR spectrum. Accordingly, it was envisioned that ${ }^{1} \mathrm{H}$ NMR spectroscopy would be a more suitable technique for the acidic $\mathrm{pH}$ titration measurements.

\subsubsection{2 ${ }^{1} H$ NMR Titrations to Determine $p K_{a}$}

Next, ${ }^{1} \mathrm{H}$ NMR spectroscopy was used to observe any changes in chemical shift for the resonances of $\mathbf{3 7}$ at very low $\mathrm{pH}$ values. Seven samples were prepared, each containing $0.5 \mathrm{~mL}$ of a $10 \mathrm{mg} / \mathrm{mL}$ solution of 37 in $\mathrm{D}_{2} \mathrm{O}$. To each of these samples, $3 \mathrm{M} \mathrm{DCl} \mathrm{(50}$ $\mu \mathrm{L}), 6 \mathrm{M} \mathrm{DCl}(50 \mu \mathrm{L}), 9 \mathrm{M} \mathrm{DCl}(50 \mu \mathrm{L})$, conc. DCl $(50 \mu \mathrm{L})$, conc. DCl (100 $\mu \mathrm{L})$, conc. $\mathrm{DCl}(150 \mu \mathrm{L})$ or conc. $\mathrm{DCl}(200 \mu \mathrm{L})$ was added to produce samples at $\mathrm{pD} 0.97,0.80$, $0.52,0.46,-0.21$ and -0.24 , respectively. A sample in $\mathrm{D}_{2} \mathrm{O}$ only $(10 \mathrm{mg} / \mathrm{mL})$ was also prepared for a measurement at $\mathrm{pD} 7$ and a separate sample in concentrated $\mathrm{DCl}$ only $(10 \mathrm{mg} / \mathrm{mL})$ was prepared to produce a $\mathrm{pD}$ of -1.00 . ${ }^{1} \mathrm{H}$ NMR spectra were then measured for each sample within two minutes of adding the $\mathrm{DCl}$, to avoid extensive hydrolysis of 37. The most basic site on glycoside 37 , and thus the site to be first protonated, was the anomeric nitrogen, as determined by the large changes in chemical shift of the $\mathrm{OMe}, \mathrm{NMe}$ and anomeric protons $(\mathrm{H}-1)$ as the $\mathrm{pD}$ decreased relative to the other proton resonances of the molecule (Figure 25). The change in chemical shift of these three resonances was then noted and examined as the $\mathrm{pD}$ decreased to later determine the $\mathrm{p} K_{\mathrm{a}}$ of the protonated anomeric nitrogen. The NAc methyl was used as an internal standard from which to measure the change in chemical shift of the other resonances as it was far enough away from the point of protonation that its chemical shift was unaffected as the $\mathrm{pD}$ was lowered. However, when using straight concentrated DCl, 3-(trimethylsilyl)propane-1-sulfonic acid (DSS) needed to be added to the sample as an external reference because the $\mathrm{N}$ acetyl group was protonated at this low $\mathrm{pD}(\mathrm{pD}=-1.00)$. DSS was also added to the sample with a pD of 7 to confirm that the $N$-acetyl resonance does not change for any of the other samples. 


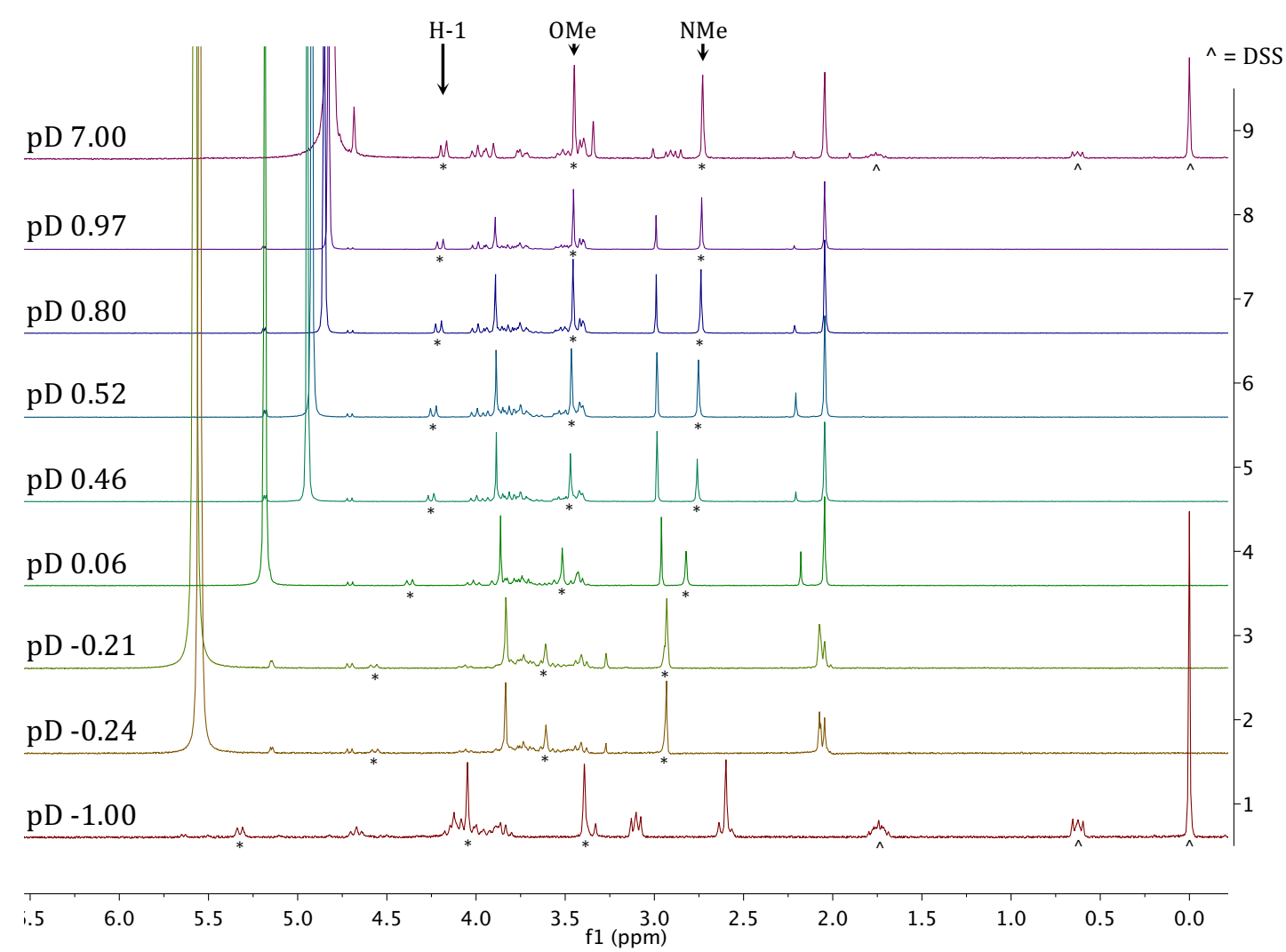

Figure 25. Stacked ${ }^{l} \mathrm{H} N M R$ spectra for the pD titrations of $37\left(\mathrm{D}_{2} \mathrm{O}, 500 \mathrm{MHz}\right)$.

To determine the $\mathrm{pD}$ of these samples, Mohr's method for the determination of chloride ion concentration ${ }^{102}$ needed to be used because the available $\mathrm{pH}$ meters could not read $\mathrm{pH}$ values below 1 or volumes as small as $0.5 \mathrm{~mL}$. $\mathrm{DCl}$ is a strong acid, thus fully dissociates to produce equal concentrations of $\mathrm{Cl}^{-}$and $\mathrm{D}^{+}$. After each sample was measured by ${ }^{1} \mathrm{H}$ NMR spectroscopy, they were then titrated against a $0.1 \mathrm{M}$ solution of $\mathrm{AgNO}_{3}$, using $\mathrm{K}_{2} \mathrm{CrO}_{4}$ as an indicator. The number of moles of $\mathrm{AgNO}_{3}$ to reach the end point of the titration (as observed by the onset of a colour change) was then used to calculate the moles of $\mathrm{D}^{+}$ions in solution, and ultimately the $\mathrm{pD}$.

The chemical shift of the NMe, OMe and H-1 protons were then each plotted against pD (Figure 26). This produced a familiar sigmoidal curve for each plot, with the $\mathrm{pK}_{\mathrm{a}}$ lying at the inflection point. ${ }^{97}$ Each plot was then fitted to equation (5) below using the least squares method, solving for $\mathrm{p} K_{\mathrm{a}}, \delta_{\mathrm{B}}$ and $\delta_{\mathrm{BH}}{ }^{+}$:

$$
\delta_{o b s}=\delta_{\mathrm{B}}+\frac{\delta_{\mathrm{BH}^{+}}-\delta_{\mathrm{B}}}{1+10^{\mathrm{pH}-\mathrm{p} K_{a}}}
$$


Where:

$\delta_{\text {obs }}=$ observed chemical shift of selected resonance.

$\delta_{\mathrm{B}}=$ chemical shift of the neutral species.

$\delta_{\mathrm{BH}}{ }^{+}=$chemical shift of the N-protonated species.

The data obtained fits equation (5) very well, with an average $\mathrm{R}^{2}$ value of 0.999 , and the $\mathrm{p} K_{\mathrm{a}}$ values solved from each plot provided an averaged value of -0.9 (NMe plot $\mathrm{p} K_{\mathrm{a}}=$ $0.9 ; \mathrm{OMe}=-1.1 ; \mathrm{H}-1=-0.8)$ (Figure 26). As observed, each resonance shows the same trend of increasing chemical shift with decreasing $\mathrm{pD}$. The chemical shift increases with protonation as electron density is withdrawn from atoms close to the site of protonation because the electrons are being donated to $\mathrm{H}^{+}$as the bond forms.

\section{${ }^{1} \mathrm{H}$-NMR Chemical Shift Dependence on pD}

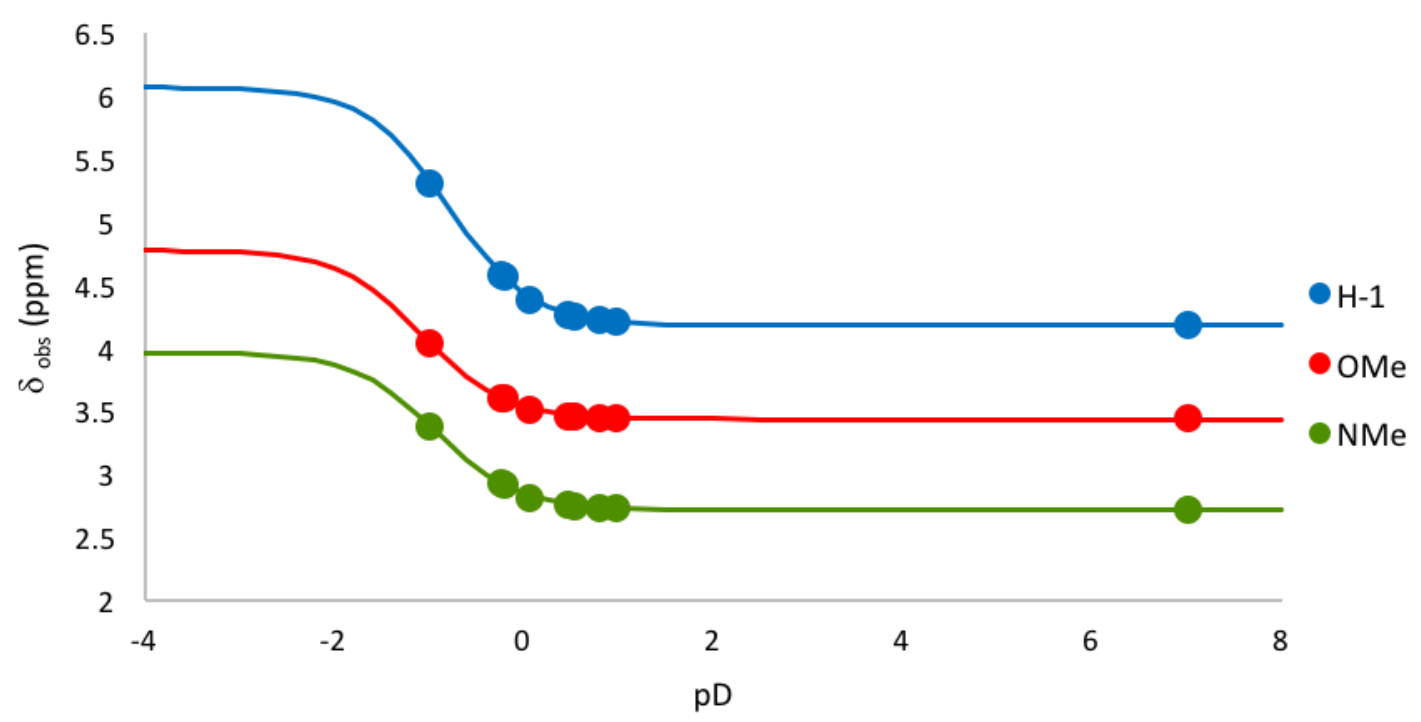

Figure 26. $p D$ dependence of ${ }^{1} H$ NMR chemical shifts of the OMe, NMe and H-1 proton resonances of 37. Each marker represents a measured data point, the line is described by equation (5), fitting $\delta_{\text {obs }}$ at different $p D$ values.

Equation (5) was defined using equations (6) to (10) below: ${ }^{100}$

Equation (6) below represents the $\delta_{\text {obs }}$ for each proton resonance as a function of both $\delta_{\mathrm{B}}$ and $\delta_{\mathrm{BH}}{ }^{+}$:

$$
\delta_{o b s}=f_{\mathrm{BH}^{+}} \delta_{\mathrm{BH}^{+}}+f_{\mathrm{B}} \delta_{\mathrm{B}}
$$


Where $f_{\mathrm{BH}}{ }^{+}$and $f_{\mathrm{B}}$ are the molar fraction of protonated and unprotonated forms respectively.

The molar fraction $f_{\mathrm{BH}}{ }^{+}$is described by the following equation:

$$
f_{\mathrm{BH}^{+}}=\frac{\left[\mathrm{BH}^{+}\right]}{\left[\mathrm{BH}^{+}\right]+[\mathrm{B}]}
$$

Considering that the molar fractions of protonated and unprotonated forms are equal to 1:

$$
f_{\mathrm{BH}^{+}}+f_{\mathrm{B}}=1
$$

equations (7) and (8) can be substituted into equation (6) to derive equation (9).

$$
\delta_{o b s}=\delta_{\mathrm{B}}+\frac{\left[\mathrm{BH}^{+}\right]}{\left[\mathrm{BH}^{+}\right]+[\mathrm{B}]} *\left(\delta_{\mathrm{BH}^{+}}-\delta_{\mathrm{B}}\right)
$$

The Henderson-Hasselbalch equation (10) can then be substituted into equation (9) to derive equation (5)

$$
\mathrm{pH}=\mathrm{p} K_{a}+\log \frac{[\mathrm{B}]}{\left[\mathrm{BH}^{+}\right]}
$$

With the knowledge that the $\mathrm{p} K_{\mathrm{a}}$ of the conjugate acid of the most basic site of glycosyloxyamine 37 is -0.9 , it can be inferred that the $\mathrm{p} K_{\mathrm{a}}$ of other, less basic sites (such as the ring oxygen), will be lower. This means that significant quantities of the protonated ring oxygen of $\mathbf{3 7}$ will only be present at a $\mathrm{pH}$ below -0.9 . This observation explains why glycosyloxyamines are so stable under basic, neutral and even mildly acidic conditions. The accepted mechanism for the hydrolysis of glycosyloxyamines involves the protonation of the ring oxygen and subsequent formation of an iminium ion rather than an oxocarbenium ion. ${ }^{43,94}$ Thus, the less basic the ring oxygen, the lower the $\mathrm{pH}$ required for the ring oxygen to become protonated. The experimentally determined $\mathrm{p} K_{\mathrm{a}}$ value for the anomeric nitrogen of the generic glycosyloxyamine $\mathbf{3 7}$ established in this thesis thus aids in explaining the stability of glycosyloxyamines. 


\subsubsection{The Mechanism of Hydrolysis of Glycosyloxyamines}

The mechanism for the formation of glycosyloxyamines is shown below (Scheme 27). Here, initial ring oxygen protonation is followed by ring opening to form the aldehyde form of the saccharide (I-III). The nitrogen of the oxyamine then attacks at the anomeric position with the elimination of water to form a glycosyloxyimine which then forms the more thermodynamically stable, ring closed glycosyloxyamine (III-VI).

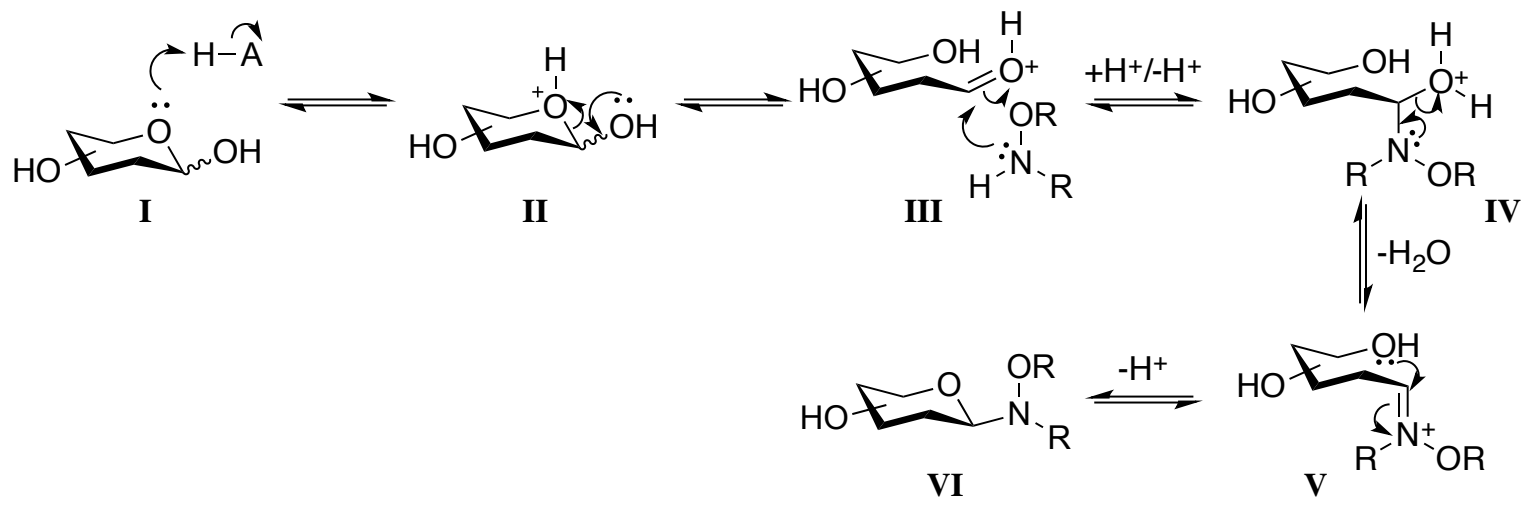

Scheme 27. The mechanism for the formation of a generic glycosyloxyamine.

It is widely accepted that the oxyamine attacks the aldehyde form of the sugar and not the oxocarbenium ion form because the leaving group on the anomeric position is not good enough for oxocarbenium formation. ${ }^{94}$ Due to the principle of microscopic reversibility, ${ }^{87}$ the mechanism of hydrolysis of glycosyloxyamines must proceed via the same pathway as their formation, as discussed earlier in this chapter (Scheme 25). The rate of hydrolysis increases with decreasing $\mathrm{pH}$, indicating that some form of acid catalysis is taking place. However, it is not conclusively known whether the hydrolysis of oxyamines occurs via SAC or GAC, and accordingly, it is not known what the RDS of the mechanism is. In the remainder of this chapter, further insight into the mechanism of glycosyloxyamine hydrolysis will be provided by considering the available literature data, as well as that obtained in this thesis.

Previous literature examining the hydrolysis of glycosyloxyamines stated that the mechanism occurs via SAC..$^{38,43,50,66}$ During SAC, the ring oxygen of the conjugate is quickly protonated in a pre-equilibrium step (VII-IX) before the slow RDS (IX-XI) (Scheme 28). Here, the RDS is the attack of water on the ring-open iminium intermediate, 
which involves general base catalysis to form the hemiaminal (XI) that then rapidly eliminates $N$-methylmethoxyamine to give the hydrolysed product (XII).
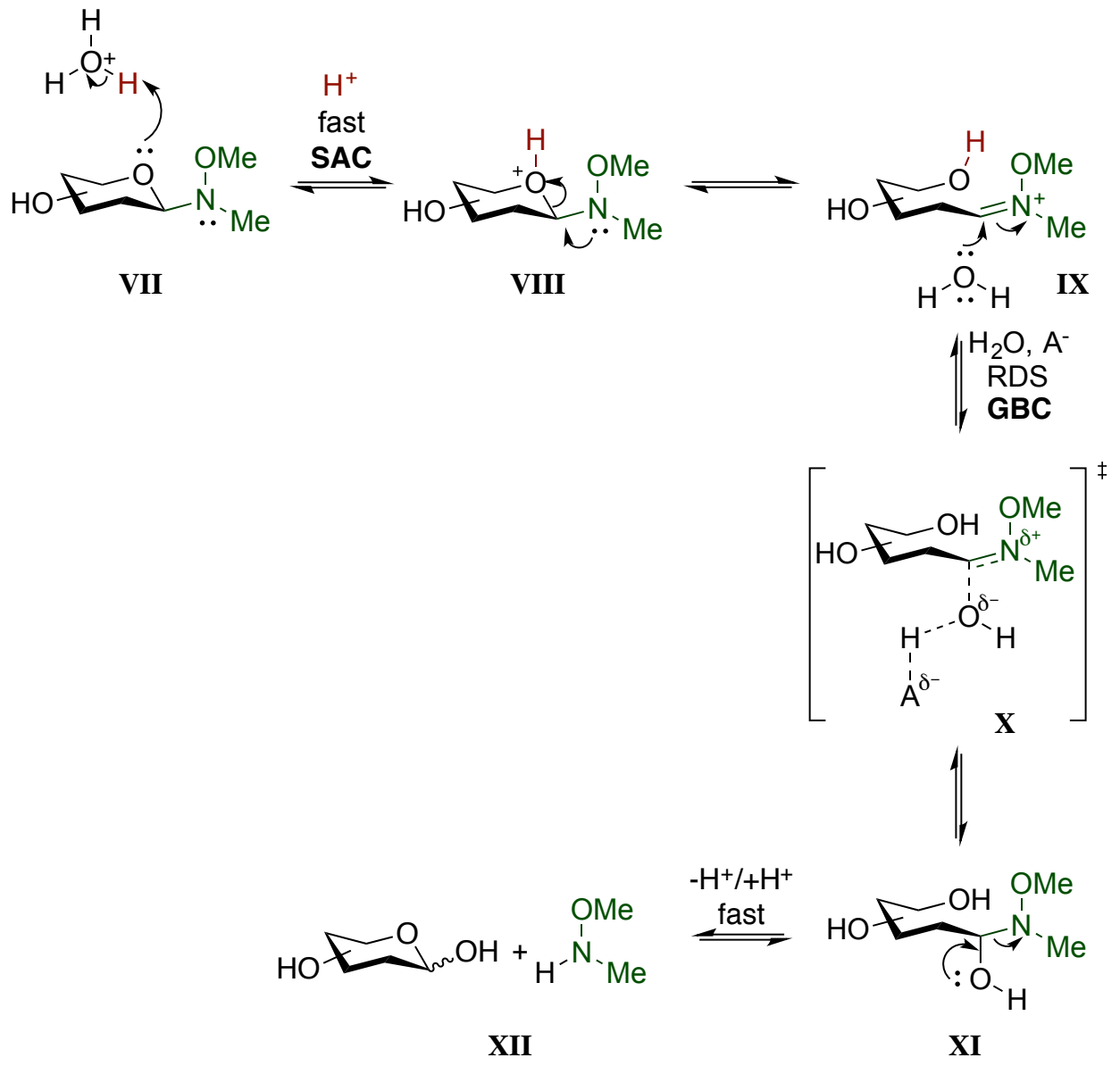

Scheme 28. Initially proposed mechanism for the hydrolysis of glycosyloxyamine 37 via specific acid catalysis followed by general base catalysis. ${ }^{43}$

An important aspect of SAC is that it is dependent on $\mathrm{pH}$ and independent of the concentration of other undissociated acids in solution. ${ }^{87}$ This is because a lower $\mathrm{pH}$ will increase the concentration of the protonated intermediates before the RDS, keeping in mind that anything which affects the steps up to and including the RDS can affect the rate of reaction. Other undissociated acids will not be strong enough to catalyse the reaction. ${ }^{87}$ Indeed, glycosylhydrazides undergo hydrolysis via SAC as evidenced in studies done by Nitz and co-workers. ${ }^{38,43,88}$ The authors conducted a hydrolysis experiment on 1glucosyl-2-phenylhydrazide at a constant $\mathrm{pH}$ of 4 with varying $\mathrm{NaOAc}$ buffer concentration and found no significant increase in the rate of hydrolysis with increasing buffer concentration, thereby concluding that the hydrolysis of hydrazides undergoes SAC rather than GAC. ${ }^{88}$ While this study may be highly indicative of the processes 
occurring during glycosyloxyamine hydrolysis, it does not meant that the two hydrolysis reactions occur via the same mechanism. Moreover, the hydrolysis experiment on 1glucosyl-2-phenylhydrazide was only conducted at a single $\mathrm{pH}$ value, bearing in mind that the RDS, and therefore the type of catalysis occurring, can vary at different $\mathrm{pH}$ values. ${ }^{87}$

A few years later, Nitz and co-workers conducted a similar hydrolysis study using selected glycosyloxyamines. ${ }^{43}$ In this study, the authors demonstrated that at $\mathrm{pH} 4-6$, buffer catalysis, consistent with GBC, is observed, with the hydrolysis still being dependent on $\mathrm{pH}$. The authors therefore concluded that at $\mathrm{pH} 4-6$, the hydrolysis of glycosyloxyamines undergoes SAC, followed by GBC as the RDS (Scheme 28). Previous literature studies have also demonstrated that the hydrolysis of oximes and hydrazones involves the rate-limiting addition of water at the $\mathrm{sp}^{2}$ carbon in the $\mathrm{pH}$ range of $4-6 .{ }^{94} \mathrm{Nitz}$ and co-workers also demonstrated that glycosyloxyamines containing electron rich sugars have increased rates of hydrolysis between $\mathrm{pH} 4-6 .{ }^{43}$ The authors state that this is because more electron rich sugars are more easily protonated. This, in turn, means that the concentration of protonated intermediates before the RDS will be higher relative to conjugates bearing more electron poor sugars under the same conditions, thereby increasing the rate of hydrolysis (moving the equilibrium in the forward direction). In addition, Cló et al. demonstrated that more electron rich $N$-substituents also increased the rate of hydrolysis of glycosyloxyamines for the same reason as described above. ${ }^{66}$ However, more electron rich sugars and/or $N$-substituents will reduce the electrophilicity of the anomeric carbon and thus should hinder the attack of water in the RDS and reduce the rate of hydrolysis. The observed increase in the rate of hydrolysis could therefore be due to the increased concentration of the protonated species having a greater effect than the reduced electrophilicity of the anomeric centre as the water molecule attacks a positively charged species, which already has a higher electrophilicity relative to the uncatalysed reaction. Notwithstanding, the observed increase in the rate of hydrolysis of electron-rich glycosyloxyamines could also be explained if GAC was occurring instead of SAC followed by GBC over this $\mathrm{pH}$ range (Scheme 29). Here, the RDS is the first step and occurs via GAC, where ring oxygen protonation and ring opening occur simultaneously (XIII-XV). Water then attacks at the anomeric centre via GBC to form the hemiaminal $(\mathbf{V})$, which eliminates $N$-methylmethoxyamine via GAC (XVII-XX) to form the hydrolysed product (XX). 


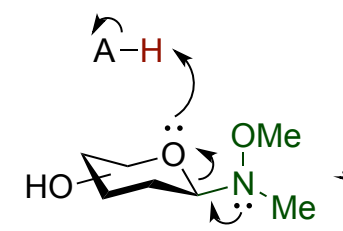

XIII
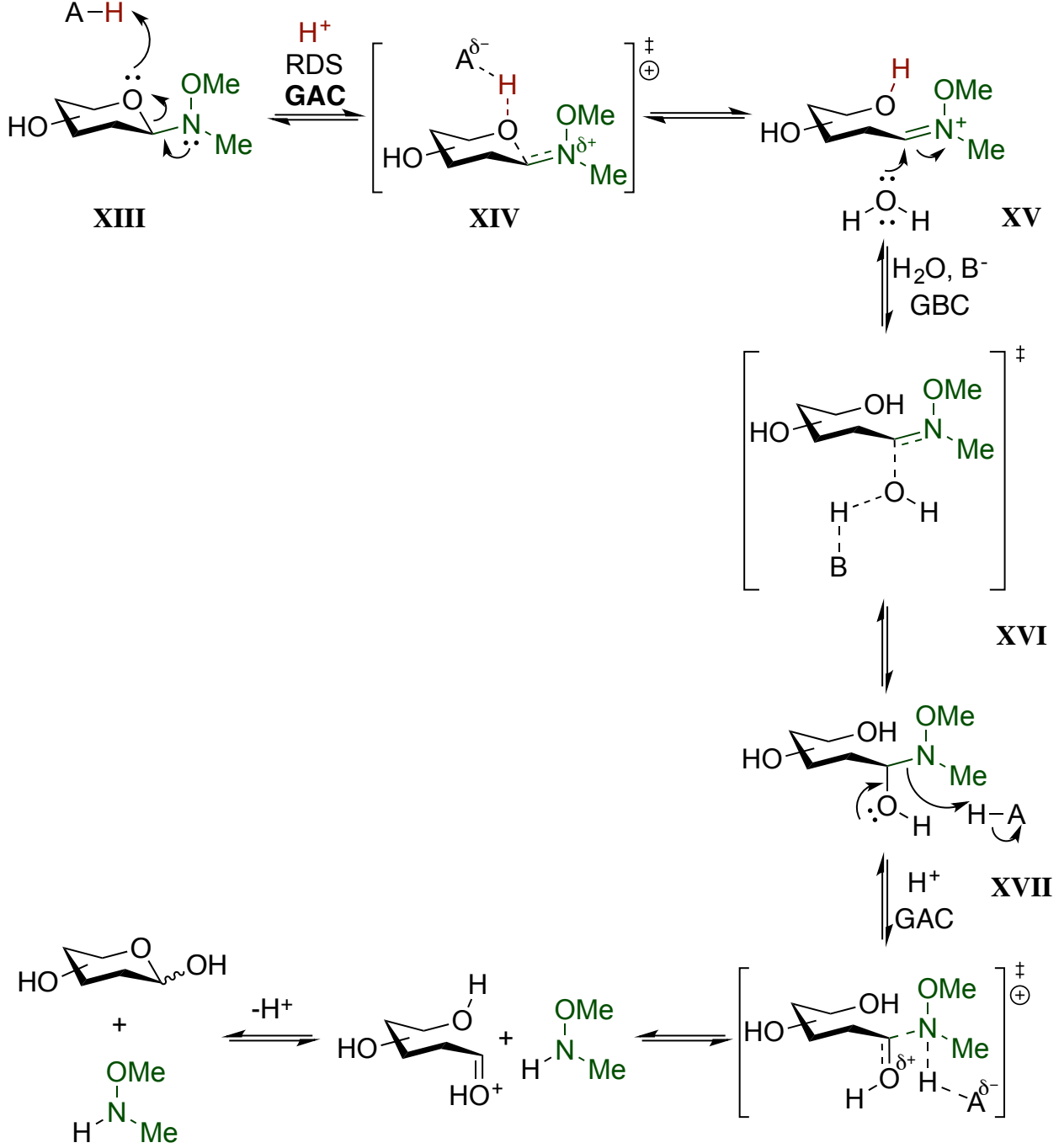

$\mathbf{X X}$

XIX

\section{XVIII}

Scheme 29. Mechanism for the hydrolysis of glycosyloxyamine 37 via general acid catalysis, as proposed in this thesis for $\mathrm{pH}=4$.

During GAC, substrate protonation by a weak acid occurs during the RDS along with other bond making/breaking processes which aid the protonation. This means that as well as being dependent on $\mathrm{pH}$, the rate of hydrolysis will also be dependent on the buffer concentration, as was observed in the literature described above, and also by work performed as part of this thesis. GAC also explains the increased rate of hydrolysis of conjugates bearing electron rich substrates. Protonation occurs during the RDS, thus the more basic electron rich substrates will hydrolyse more quickly. It is likely that the attack of water is GBC and the loss of the oxyamine is GAC, as illustrated in the scheme above. However, it is unlikely that either of these would be the RDS if the initial protonation is 
GAC in the pH range 4-6. Nitz and co-workers suggests that the loss of the oxyamine would be a fast process as the attack of oxyamines on aldehydes is fast and reversible. ${ }^{43}$ The GBC step is also unlikely to be the RDS at this $\mathrm{pH}$ range, as more electron rich substrates would decrease the rate of reaction rather than increase it. The hydrolysis studies conducted in Chapter two of this thesis have demonstrated that a significant increase in the rate of hydrolysis is observed when the buffer concentration is increased at $\mathrm{pH}$ 4. This indicates that either GAC or GBC is occurring, which could fit either mechanistic model (Scheme 28 and Scheme 29). Unfortunately, these two mechanisms are indistinguishable kinetically. This is because mechanisms which involve HA (i.e. GAC) cannot be kinetically distinguished from mechanisms involving $\mathrm{H}^{+}$and $\mathrm{A}^{-}$ (i.e. SAC together with GBC). ${ }^{94}$ That said, SAC is only effective when the $\mathrm{pH}$ of solution is at or below the $\mathrm{p} K_{\mathrm{a}}$ of the conjugate acid of the base that is being protonated. ${ }^{87} \mathrm{By}$ determining that the $\mathrm{p} K_{\mathrm{a}}$ of the conjugate acid of glycosyloxyamine 37 (Figure 22) is -0.9 , we know that the $\mathrm{p} K_{\mathrm{a}}$ of the protonated ring oxygen must be lower, because the ring oxygen is less basic than the anomeric nitrogen. ${ }^{94}$ Accordingly, this suggests that SAC may not be effective in the $\mathrm{pH}$ range 4-6, and that therefore the GAC mechanism (Scheme $29)$ is more likely.

The RDS of hydrolysis, and therefore whether GAC or SAC + GBC is occurring, can also vary with $\mathrm{pH}$. Evidence obtained in the literature and in this thesis towards understanding where the RDS of hydrolysis is, has only been obtained between $\mathrm{pH}$ 4-6. However, while conducting the ${ }^{1} \mathrm{H}$ NMR $\mathrm{pD}$ titration experiment for the determination of the $\mathrm{p} K_{\mathrm{a}}$ of $\mathbf{3 7}$, it was noticed that the conjugate appeared to be reasonably stable at $\mathrm{pD}=-1.0$. Evidence of this can be seen in the ${ }^{1} \mathrm{H}$ NMR spectrum where the molecule appears to be largely intact, with almost no indication of the GlcNAc degradation product (Figure 25). This could be an indication of a change of RDS in the mechanism from GAC at $\mathrm{pH} 4-6$ to SAC $+\mathrm{GBC}$ at $\mathrm{pH}$ values close to -1 . At such a low $\mathrm{pH}$, the protonation of the substrate can no longer be rate limiting as the concentration of $\mathrm{H}^{+}$ions in solution is so high that almost every substrate molecule will be quickly protonated. This implies that SAC is now occurring at $\mathrm{pH}=-1.0$, followed by the slow RDS, which requires GBC. In such acidic conditions, the attack of water in the RDS will be substantially slower as there are very few $\mathrm{H}_{2} \mathrm{O}$ molecules to act as a base and catalyse the reaction. Also, SAC is more likely to be effective at $\mathrm{pH}-1$ rather than $\mathrm{pH} 4-6$ as the $\mathrm{pH}$ is now close to the $\mathrm{p} K_{\mathrm{a}}$ of the protonated conjugate. 
One method to distinguish between the two mechanisms (Scheme 28 and Scheme 29) is by examining kinetic isotope effects on the rate of hydrolysis. As discussed in section 3.1.2, SAC involves an inverse solvent isotope effect $\left(k\left[\mathrm{D}_{2} \mathrm{O}\right]>k\left[\mathrm{H}_{2} \mathrm{O}\right]\right)$, while GAC involves a normal kinetic isotope effect $\left(k_{\mathrm{H}}>k_{\mathrm{D}}\right)$. The presence of SAC can therefore be determined by conducting the hydrolysis reaction in $\mathrm{H}_{2} \mathrm{O}$ and $\mathrm{D}_{2} \mathrm{O}$ separately, and monitoring any effect on the rate caused by the deuterated $v s$. non-deuterated solvents. This experiment was attempted on glycosyloxyamine $\mathbf{3 7}$ using polarimetry to monitor the rate of hydrolysis. Glycosyloxyamine 37 has an optical rotation of $\alpha_{\mathrm{D}}{ }^{20.1}=-10(c=0.1$, $\mathrm{MeOH})$. The rate of hydrolysis can be monitored as the optical rotation reduces to 0 due to the hydrolysis of $\mathbf{3 7}$ and the formation of the achiral free oxyamine and the mixture of $\alpha$ - and $\beta$-GlcNAc epimers. Unfortunately, the available polarimeter was not accurate enough to monitor this change at $\mathrm{pH} 4$. A future prospect of this study would be to obtain the use of a more accurate polarimeter and re-investigate this experiment.

\subsection{Conclusion}

The $\mathrm{p} K_{\mathrm{a}}$ of the conjugate acid of a generic glycosyloxyamine was successfully determined to be -0.9 using ${ }^{1} \mathrm{H}$ NMR titrations. This value corresponds to the $N$-protonated form of the glycosyloxyamine and indicates that the anomeric nitrogen is the most basic site on the conjugate. It is thus inferred that the less basic ring oxygen will have a $\mathrm{p} K_{\mathrm{a}}$ lower than -0.9. Such a low $\mathrm{p} K_{\mathrm{a}}$ indicates that glycosyloxyamines require a low $\mathrm{pH}$ to become protonated, which explains their high stability under basic, neutral and mildly acidic conditions. Previous literature has suggested that the hydrolysis of these conjugates is SAC, but studies herein suggest that GAC may be occurring and that the type of acid catalysis is likely to change with $\mathrm{pH}$. More studies, however, would be required to better determine the mechanism of hydrolysis for these conjugates. 


\section{Chapter 4}

\section{Synthesis and Application of Thiol Functionalised Oxyamine Linkers}

\subsection{Introduction}

Bifunctional oxyamine linkers are a versatile tool for the conjugation of saccharides to a variety of different substrates. The $\mathrm{N}, \mathrm{O}$-dialkyl functionality of the oxyamine allows for direct conjugation to the reducing end of saccharides, thereby forming a stable glycosidic bond without the need for activating groups or protecting groups. Thus, both synthetically- and biologically-derived sugars can be conjugated using this linker. ${ }^{53}$ Moreover, glucose derived saccharides with a free reducing end will exclusively form the $\beta$-pyranose product. ${ }^{50}$ Using the reactive functional group on the terminal end of the linker, the resulting glycoconjugate can then be linked to a variety of substrates including proteins, ${ }^{16}$ dendrimers, ${ }^{23}$ nanoparticles, ${ }^{24}$ and microarray slides. ${ }^{18}$ For example, a thiol functionalised oxyamine linker can be used to link sugars to the surface of gold nanoparticles, ${ }^{24}$ and glycoproteins can also be produced by forming disulfide bonds with cysteine residues in proteins. ${ }^{103}$

\subsubsection{Gold Nanoparticles as a Multivalent Display Scaffold}

A major constraint with the use of carbohydrates as ligands for lectins is that their binding interactions with their lectin counterparts on cell surfaces is often too weak to induce cellular activation. ${ }^{77}$ However, by placing multiple target carbohydrates on a multivalent display scaffold, the local concentration of the carbohydrate is dramatically increased. This facilitates the crucial cross-linking on the cell surface that is required for cellular activation. Such multivalent display scaffolds can also be used to label the glycocluster, thereby allowing its distribution in a biological system to be monitored. ${ }^{81}$

As discussed in the introduction of this thesis, gold nanoparticles offer advantages over other multivalent display scaffolds. They are relatively easy to synthesise and purify, and their size and shape can be finely controlled using the appropriate literature 
methodology. ${ }^{81,82}$ They are also larger than proteins and dendrimers, which allows them to be more easily visualised during phagocytosis by macrophages or DCs using optical microscopy. Glycosylated gold nanoparticles (AuNPs) can easily be synthesised by adding the glycoside functionalised with a terminal thiol to the reaction mixture during AuNP synthesis. The glycoside then forms a self assembled monolayer (SAM) on the surface of the AuNP via strong thiolate-Au bonds $\left(40-50 \mathrm{kcal} \mathrm{mol}^{-1}\right){ }^{104}$ Several glycosylated AuNPs have been prepared to date and include $N$ - $\alpha$-fucosyl- $\beta$-alanylamidefunctionalised, Lewis ${ }^{\mathrm{X}}\left(\mathrm{Le}^{\mathrm{X}}\right)$-functionalised, and Mano1-2Man $\alpha$-functionalised AuNPs as targets for the DC-SIGN receptor on DCs. ${ }^{24,105}$ Man $\alpha 1-2$ Man $\alpha$-functionalised AuNPs mimic the high mannose clusters found on the surface of HIV and competitively inhibit the binding of the virus to DC-SIGN. ${ }^{105}$

The objective of this chapter is to prepare an oxyamine dithiol linker, which can be functionalised with glycans, such as chitin. It is known that bidentate dithiol ligands form more stable AuNPs compared to their monodentate counterparts. ${ }^{83,106}$ For example, Mei et al. demonstrated that ligands with higher coordination numbers are better able to stabilise AuNPs against excess added salt, which causes the nanoparticles to aggregate. These ligands are also more resilient to displacement by dithiothreitol (DTT) than ligands with only one coordination site. ${ }^{83}$ In a different study, Mei et al. also concluded that AuNPs bearing dithiol ligands were more stable over a wide $\mathrm{pH}$ range and at high ionic concentrations than monodentate ligands, making them effective tools for use in

biological systems where high ionic concentrations and changes in $\mathrm{pH}$ are common. ${ }^{106}$ Accordingly, glycosylated AuNPs formed using a dithiol linker should have good aqueous dispersion, be stable in biological conditions, and be more resistant to displacement of the glycosyl-ligands by other thiol functionalised biomolecules.

\subsection{Results and Discussion}

\subsubsection{Sulfur Functionalised Linker Design and Synthesis}

Glycoconjugates formed from both "Type A" and "Type B" oxyamine linkers show comparable hydrolytic stability, as determined in Chapter two of this thesis. However, the methodology for the synthesis of the "Type B" linker is not only more efficient than that of the "Type A" linker, but is also more versatile and readily allows for the synthesis 
of linkers with different terminal functional groups. ${ }^{53}$ The "Type B" oxyamine linker synthesis was therefore adapted for the design and synthesis of an oxyamine linker bearing terminal thiol groups for the multivalent display of glycans on gold nanoparticles.

To synthesise the target dithiol functionalised "Type B" oxyamine linker 39, it was envisioned that trithiol 38 could react with acrolein (Scheme 30), in a manner similar to the strategy developed by Timmer and co-workers for the synthesis of the analogous monodentate thiol oxyamine linker. ${ }^{53}$ The use of a $\mathrm{C}_{3}$ symmetric trithiol, such as 1,1,1tris(mercaptomethyl)ethane (38), alleviates concerns about the regioselectivity of the thiol addition to acrolein. Tris(mercaptomethyl)ethane (38) was in turn envisioned to be avaliable from tris(hydroxymethyl)ethane (40) via per-bromination and displacement of the bromine groups with sulfur.

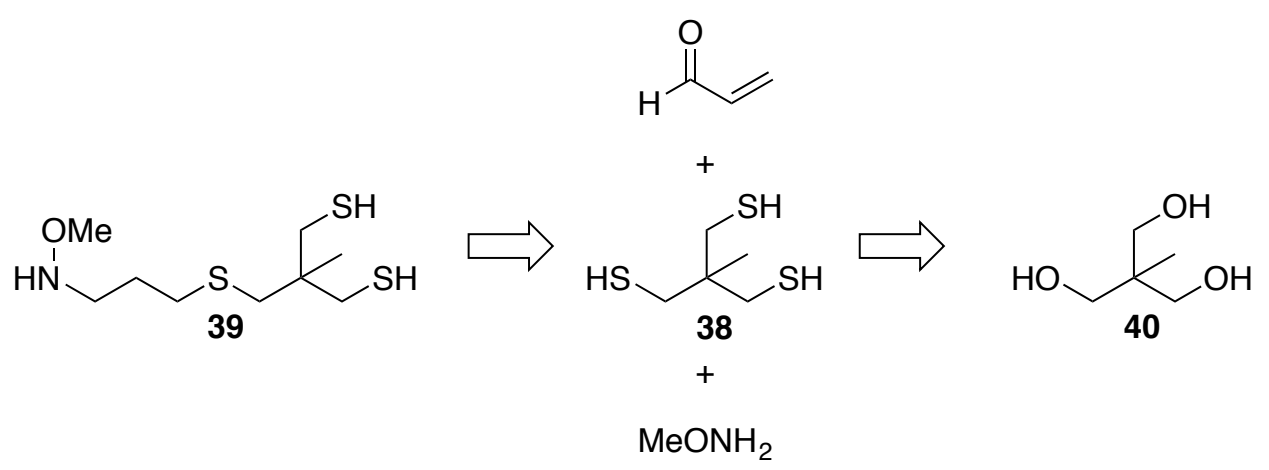

Scheme 30. Retrosynthetic analysis for the synthesis of "Type B" linker 39.

\subsubsection{Trithiol Synthesis}

To commence the synthesis of trithiol $\mathbf{3 8}$, it was initially proposed that triol $\mathbf{4 0}$ could be treated with $\mathrm{PBr}_{3}$ according to a literature procedure ${ }^{107}$ and the corresponding tribromo derivative 41 substituted using thiourea. Thiourea was the preferred thiolating agent as this reaction has been reported to be straightforward, high yielding, and can be completed under atmospheric conditions. ${ }^{108}$ Literature precedent suggested that the transformation of triol $\mathbf{4 0}$ to tribromide $\mathbf{4 1}$ could be achieved in a $40 \%$ yield via the treatment of $\mathbf{4 0}$ with 1.5 equivalents of $\mathrm{PBr}_{3}$ in $\mathrm{MeCN}$ at reflux for 24 hours. ${ }^{107}$ Accordingly, 1.5 equivalents of $\mathrm{PBr}_{3}$ was reacted with $\mathbf{4 0}$ in freshly distilled MeCN for 24 hours (Table 1, entry 1). Upon work-up and purification of the reaction mixture by silica gel flash chromatography, ${ }^{1} \mathrm{H}$ and ${ }^{31} \mathrm{P}$ NMR spectroscopy revealed that $\mathbf{4 0}$ had reacted to form a 
cyclic phosphate. The ${ }^{13} \mathrm{C}$ NMR spectrum revealed three carbon resonances, all of which showed carbon-phosphorus coupling $\left(125 \mathrm{MHz}, \mathrm{CDCl}_{3}, \delta 78.9\left(\mathrm{~d},{ }^{2} J_{P, C}=5.7 \mathrm{~Hz}\right.\right.$, $\left.\left.\mathrm{CH}_{2} \mathrm{OP}\right), 34.3\left(\mathrm{~d},{ }^{3} J_{P, C}=38.6 \mathrm{~Hz}, \mathrm{Cq}\right), 13.1\left(\mathrm{~d},{ }^{4} J_{P, C}=2.6 \mathrm{~Hz}, \mathrm{CH}_{3}\right)\right)($ Figure 27$) .{ }^{1} \mathrm{H}$ NMR spectra indicated that the $\mathrm{CH}_{2}$ protons had shifted downfield to $\delta 4.48$ compared to the trihydroxy starting material at $\delta 3.47$, which is consistent with the attachment of a more electron-withdrawing group. The $\mathrm{CH}_{2}$ proton resonance also showed proton-phosphorus coupling at $\delta 4.48\left(\mathrm{~d},{ }^{3} J_{P, H}=6.4 \mathrm{~Hz}\right) .{ }^{31} \mathrm{P}$ NMR spectroscopy indicated the presence of a single phosphorus resonance at $\delta-8.2\left(121 \mathrm{MHz}, \mathrm{CDCl}_{3}\right)$, which, along with the ${ }^{13} \mathrm{C} \mathrm{NMR}$ data, is indicative of a cyclic phosphate. ${ }^{109}$ HRMS also concluded the formation of the phosphate $\left(\mathrm{m} / z\right.$ calcd. for $\left[\mathrm{C}_{5} \mathrm{H}_{10} \mathrm{O}_{4} \mathrm{P}\right]^{+}: 165.0311$, obsd.: 165.0303).

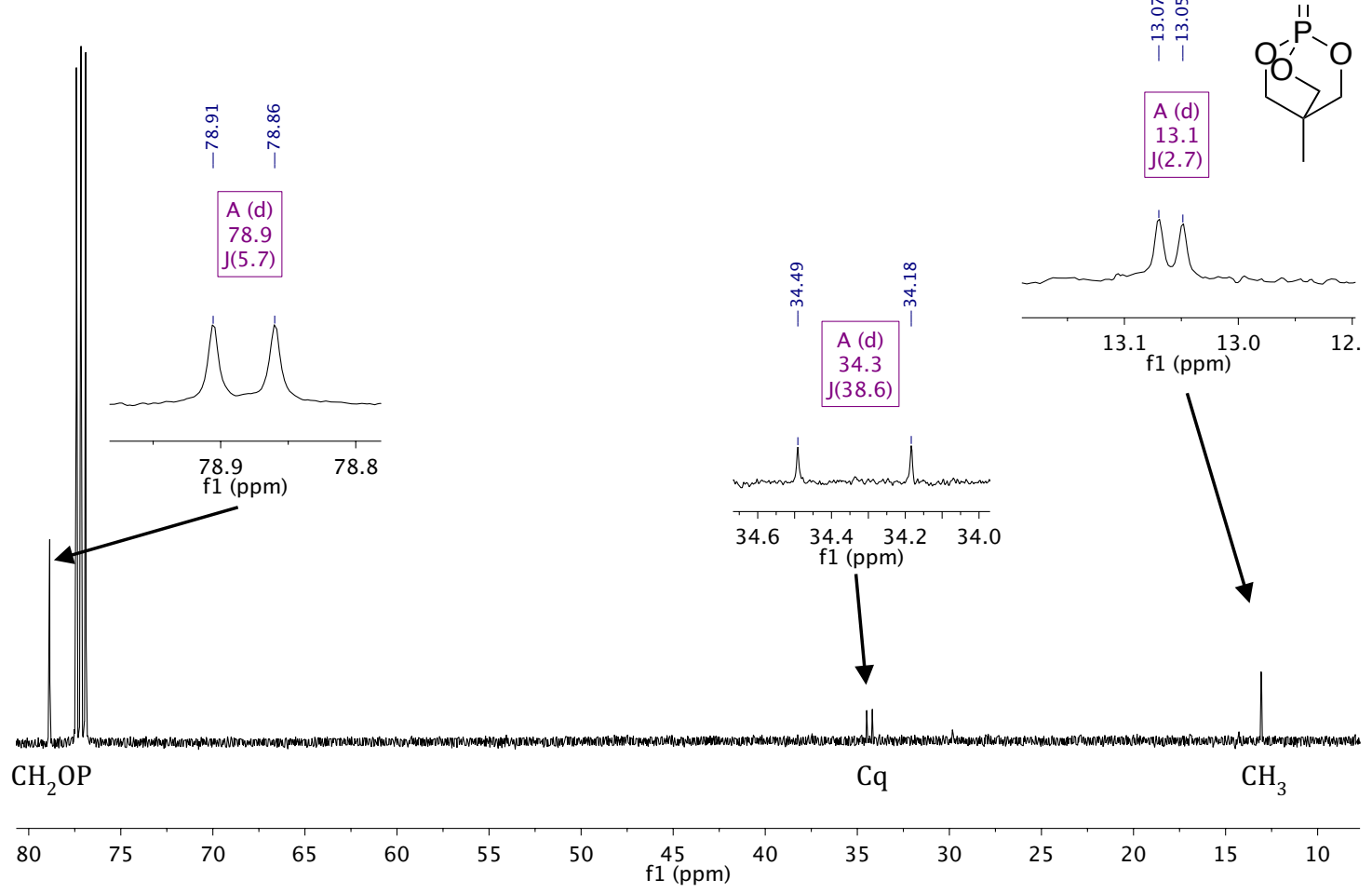

Figure 27. ${ }^{13} \mathrm{C} \mathrm{NMR}$ spectrum of cyclic phosphate $\left(\mathrm{CDCl}_{3}, 125 \mathrm{MHz}\right)$, inserts are magnifications of each resonance illustrating $J_{C, P}$ coupling constants.

In an attempt to prevent phosphate formation and form the desired product, $\mathrm{MeCN}$ was replaced with the higher boiling pyridine as the solvent in the next reaction and more equivalents of $\mathrm{PBr}_{3}$ (3 eq.) were added (Table 1, entry 2). Unfortunately, the same phosphate was the only product observed by TLC $\left(\mathrm{R}_{f}=0.25(\right.$ EtOAc/PE, 3:1, v/v)), despite refluxing the reaction for 48 hours; it was subsequently isolated following workup. In the next reaction, ten equivalents of $\mathrm{PBr}_{3}$ were used, which produced the desired 
product 41 in a $27 \%$ yield after purification by silica gel flash chromatography (entry 3). The formation of $41\left(\mathrm{R}_{f}=0.92(\mathrm{PE} / \mathrm{EtOAc}, 3: 1, \mathrm{v} / \mathrm{v})\right)$ was difficult to monitor by TLC as it could only be stained at a relatively high concentration by development with potassium permanganate, which presented a challenge, especially during purification. The ${ }^{1} \mathrm{H}$ and ${ }^{13} \mathrm{C}$ NMR spectra of $\mathbf{4 1}$ matched those reported in the literature ${ }^{107}$ with the $\mathrm{CH}_{2}-\mathrm{Br}$ proton resonances appearing as a singlet at $\delta 3.49$ and the methyl protons appearing as a singlet at $\delta 1.28\left(\mathrm{CDCl}_{3}, 500 \mathrm{MHz}\right)$. The $\mathrm{CH}_{2}-\mathrm{Br}$ carbon resonances had a chemical shift of $\delta 39.0$ and the methyl carbon appeared at $\delta 21.6$, while the quaternary carbon was present at $\delta 39.6\left(\mathrm{CDCl}_{3}, 125 \mathrm{MHz}\right)$. In theory, only one equivalent of $\mathrm{PBr}_{3}$ is required to substitute all the hydroxyls of $\mathbf{4 0}$ for bromines, forming $\mathrm{P}(\mathrm{OH})_{3}$ as a by-product, however as described above, further equivalents were required to ensure the reaction went to completion. In an attempt to further increase the yield of the target product, $\mathbf{4 0}$ was refluxed in $\mathrm{HBr}$ and $\mathrm{H}_{2} \mathrm{SO}_{4}$, according to a literature procedure used for the synthesis of 2-(bromomethyl)-1,4-dibromobutane. ${ }^{110}$ However, despite the harsh reaction conditions, only mono- and di-bromo products were observed (entry 4 ).

Table 1. Development of reaction conditions for the synthesis of 41 from 40.

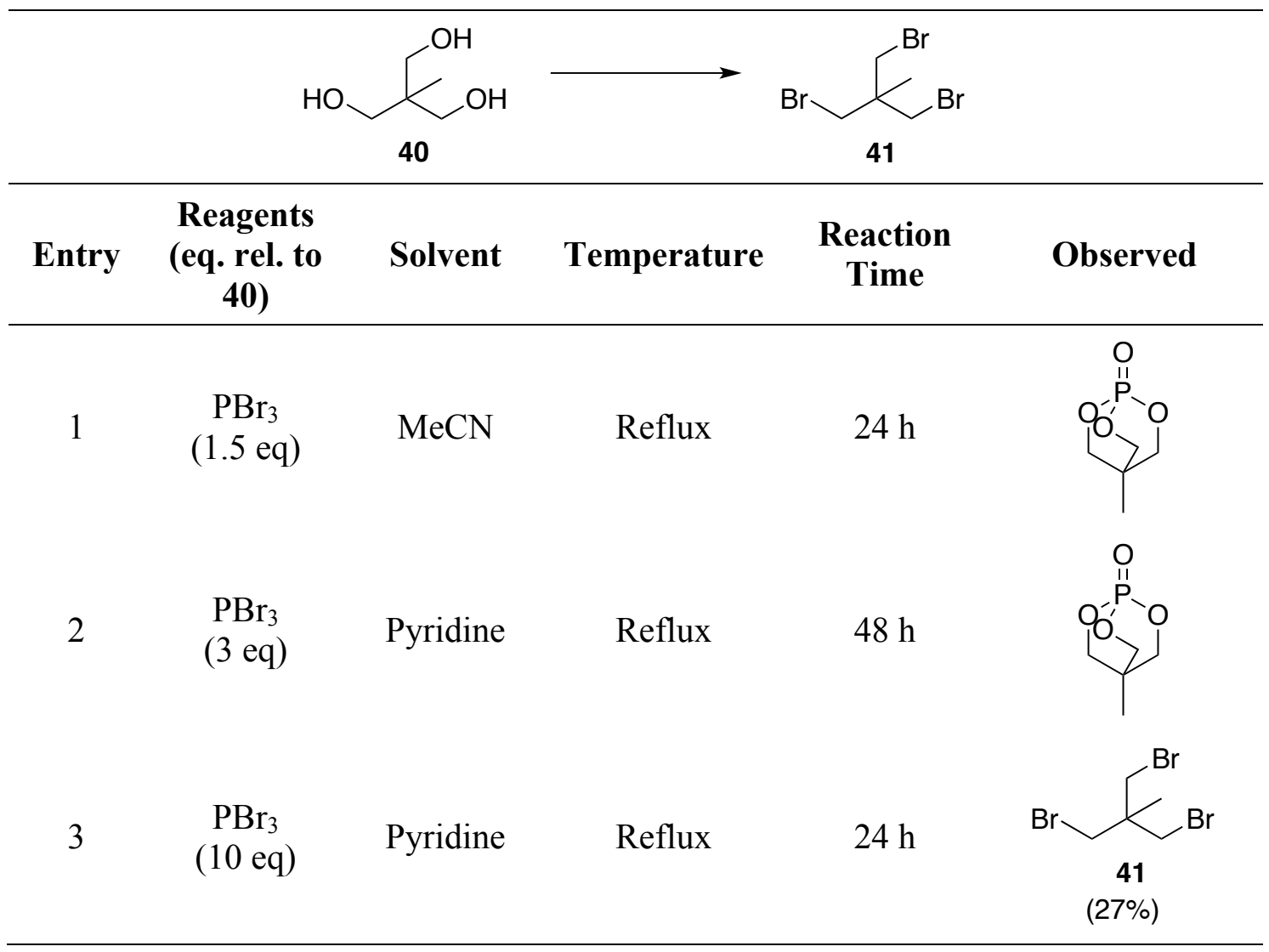




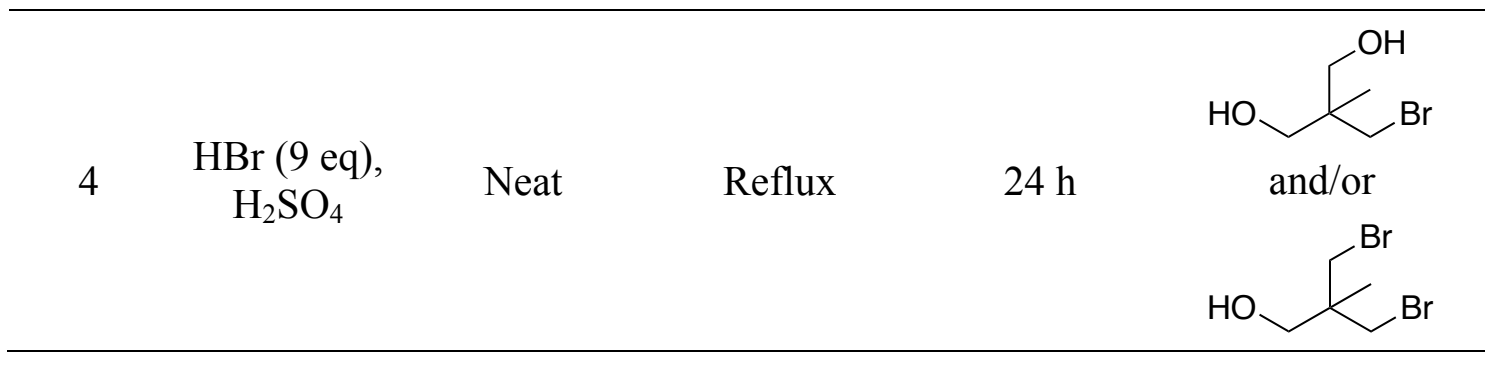

With tribromide 41 in hand, attempts were then made to convert this to trithiol 38. A Japanese patent indicated that 2,2-bis(mercaptomethyl)-1,3-propanedithiol could be synthesised from its tetrabromo counterpart using thiourea. ${ }^{111}$ Accordingly, 41 was reacted with 3.5 equivalents of thiourea in $\mathrm{EtOH}$ at reflux for 3 hours, aqueous $\mathrm{NaOH}$ $(2.5 \mathrm{M}, 0.2 \mathrm{~mL})$ was then added to the reaction mixture, and the reaction refluxed for a further 2 hours (Scheme 31). Unfortunately, no desired product was observed and only thiourea and $\mathbf{4 1}$ were returned.

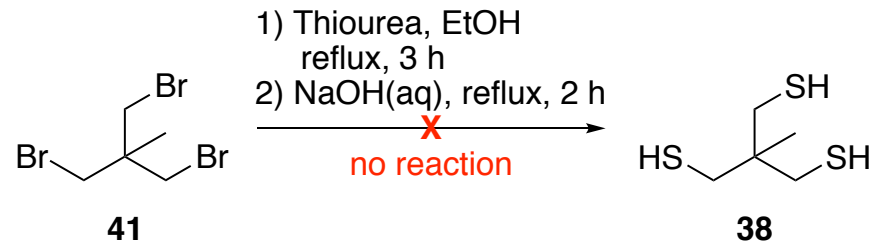

Scheme 31. Attempted synthesis of trithiol 38 from tribromide 41.

To ensure that the reaction conditions were feasible, an attempt was then made to transform a simpler dibromo substrate into a dithiol using thiourea. Dibromobutane (42) was thus reacted with thiourea ( 2.2 eq.), using the same reaction conditions described above, to yield butanedithiol (43) in a 73\% yield without any need for further purification (Scheme 32).

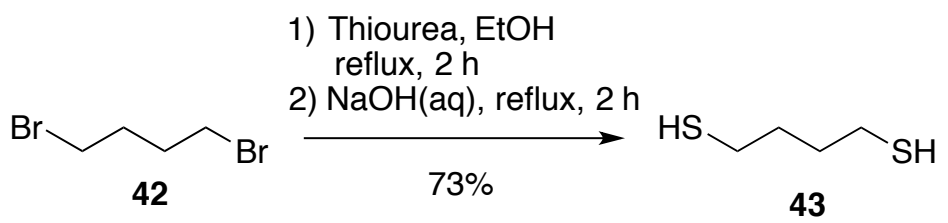

Scheme 32. Synthesis of butanedithiol (43) from dibromobutane (42) using thiourea.

From these observations, it was thus thought that $\mathbf{4 1}$ may be too sterically hindered to allow for substitution of the bromine atoms by thiourea and the formation of the isothiouronium ion intermediate. Accordingly, it was proposed that tribromopropane (45) 
would serve as a better scaffold as this substrate is slightly less sterically hindered and will nonetheless still provide the desired terminal dithiol functionality once converted into the oxyamine linker. Moreover, propanetrithiol has also been specifically synthesised from 45 using thiourea according to a Chinese patent. ${ }^{112}$ To this end, tribromopropane (45) was synthesised by reacting allylbromide (44) with $\mathrm{Br}_{2}$ in carbon tetrachloride (Scheme 33).

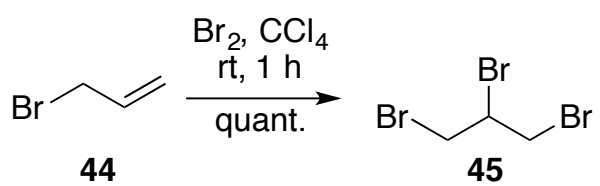

Scheme 33. Synthesis of tribromopropane (45).

Unfortunately, attempts to synthesise propanetrithiol from 45 using thiourea were unsuccessful (Scheme 34). Very little material was recovered in the organic layer following extraction and ${ }^{1} \mathrm{H}$ NMR analysis of this material presented multiple broad resonances, which were difficult to interpret and indicated that the formation of a polymer had occurred. No tribromopropane was re-isolated in the organic layer. Indeed, Russavskaya et al. described the formation of a strongly cross-linked disulfide dendrimeric polymer en route to the synthesis of 1,2,3-propanetrithiol from 1,2,3trichloropropane. ${ }^{113}$ The authors reduced this polymer to form the trithiol using an alkaline solution of hydrazine hydrate, followed by acidification. In view of this, the crude reaction mixture was then subjected to Russavskaya's reduction conditions in an attempt to reduce any polymer that may have been formed. Unfortunately, this did not generate product and the same crude residue was returned.

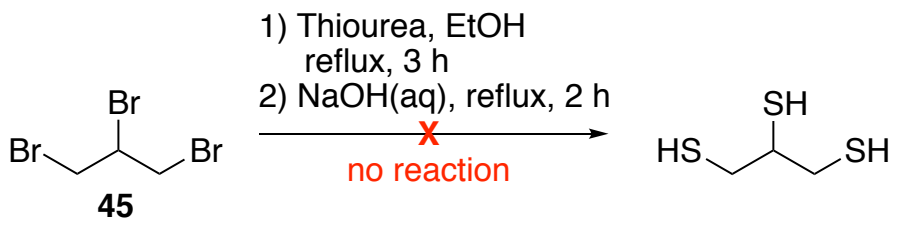

Scheme 34. Attempted synthesis of 1,2,3-propanetrithiol from tribromide 45.

Following little success in the synthesis of propanetrithiol from 45, alternative methodologies for the synthesis of terminal thiols were then explored. Guyon et al. synthesised a dithiol from 1,2-dibromodecane (46) (Scheme 35), ${ }^{114}$ whereby they reacted 
46 with mercaptoethanol in the presence of sodium ethoxide to produce diol $\mathbf{4 7}$ in modest yield. The diol was then refluxed with thiourea in concentrated $\mathrm{HCl}$, followed by hydrolysis using $\mathrm{NaOH}$ to produce dithiol 48.

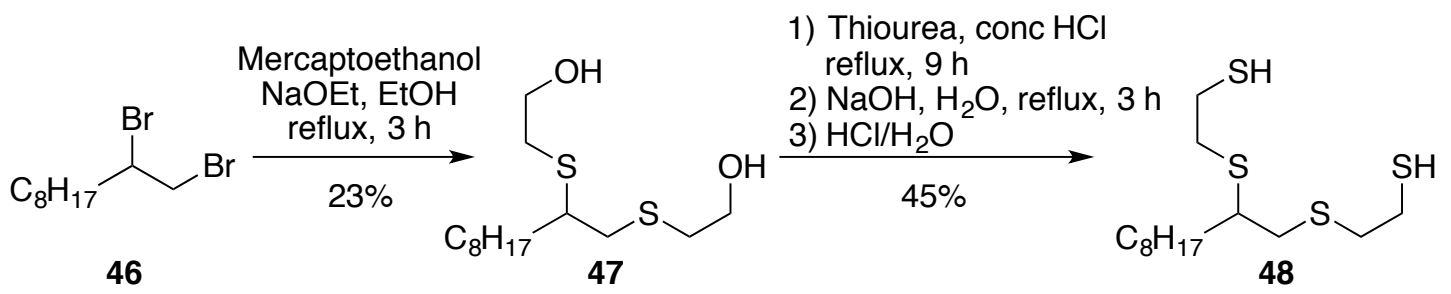

Scheme 35. Guyon's synthesis of dithiol $48 .^{114}$

It was thought that the above procedure could be applied to the thiolation of $\mathbf{4 5}$ as the addition of mercaptoethanol to $\mathbf{4 5}$ would generate a longer alkyl chain, thereby facilitating the subsequent reaction with thiourea. Thus, a pilot study was conducted whereby $\mathbf{4 2}$ was refluxed for 3 hours with mercaptoethanol in the presence of sodium ethoxide (Scheme 36). The resulting diol 49 was isolated and placed in a solution of thiourea in concentrated $\mathrm{HCl}$ and refluxed for 2 hours before being hydrolysed by the addition of aqueous $\mathrm{NaOH}$. Acidification of the crude mixture produced dithiol $\mathbf{5 0}$ in a moderate $(50 \%)$ yield.

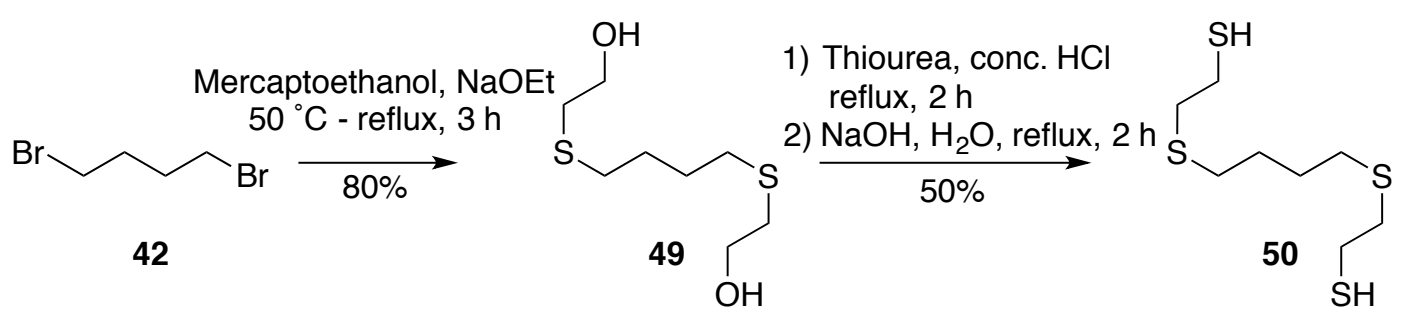

Scheme 36. Dithiolation of dibromobutane (42) using mercaptoethanol and thiourea.

Following this success, the reaction conditions were then applied to 45 (Scheme 37). Here, tribromide $\mathbf{4 5}$ was dissolved in a solution containing mercaptoethanol and sodium ethoxide in ethanol, refluxed for 3 hours, and the reaction mixture then extracted using $\mathrm{CH}_{2} \mathrm{Cl}_{2}$. A ${ }^{1} \mathrm{H}$ NMR spectrum of the material from the crude reaction mixture revealed the formation of elimination product $\mathbf{5 1}$ instead of the desired triol. The ${ }^{1} \mathrm{H}$ NMR spectrum of 51 matched the literature ${ }^{115}$ with characteristic proton resonances at $\delta 5.79$ and 5.52 (methylene protons), and a singlet at $\delta 3.45$ belonging to the $\mathrm{CH}_{2}$ protons adjacent to the double bond. 


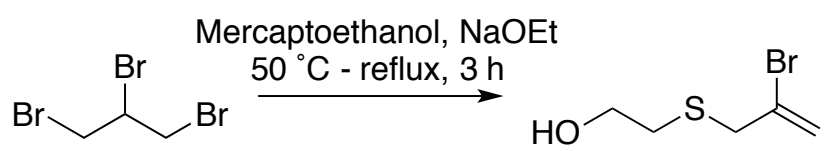

45

51

Scheme 37. Reaction of 45 with mercaptoethanol producing the undesired elimination product 51.

To prevent the formation of the elimination product $\mathbf{5 1}$, the reaction was repeated under milder conditions. First, NaOEt was replaced with $\mathrm{NaHCO}_{3}$ and the reaction mixture stirred at $40{ }^{\circ} \mathrm{C}$ overnight, however only tribromopropane was recovered. The reaction was then attempted using $\mathrm{NaOH}$ with stirring at room temperature for 5 hours. This also produced the elimination product 51. Another attempt was made using $\mathrm{K}_{2} \mathrm{CO}_{3}$ as the base with stirring at room temperature. TLC analysis after 15 minutes, however, revealed that 51 had formed $\left(\mathrm{R}_{f}=0.71(\mathrm{PE} / \mathrm{EtOAc}, 1: 1, \mathrm{v} / \mathrm{v})\right)$, which indicates that $\mathbf{4 5}$ is very prone to elimination and that the optimisation of this reaction would be challenging.

Following these unsuccessful attempts at synthesising a variety of different trithiols, alternative methodology for the synthesis of the originally proposed tris(mercaptomethyl)ethane (38) was then re-examined. To this end, Valiulin et al. had used a mixture of $\mathrm{NaSH} / \mathrm{S}$ to produce $\mathbf{3 8}$ from tris(tosyloxymethyl)ethane. ${ }^{116}$ In addition, Petrovski et al. ${ }^{117}$ had reacted tris(hydroxymethyl)ethane (40) with benzenesulfonyl chloride in pyridine to yield tris(benzenesulfonylmethyl)ethane (52), which is similar to tris(tosyloxymethyl)ethane. Thus, it was proposed that a combination of these two methodologies would allow for the successful synthesis of tris(mercaptomethyl)ethane (38). Accordingly, tris(hydroxymethyl)ethane (40) was treated with benzenesulfonyl chloride in pyridine and benzenesulfonate $\mathbf{5 2}$ was precipitated from the reaction mixture using a solution of concentrated $\mathrm{HCl}$, water and methanol, and obtained in excellent yield $(96 \%)$ with no need for further purification (Scheme 38$)$.
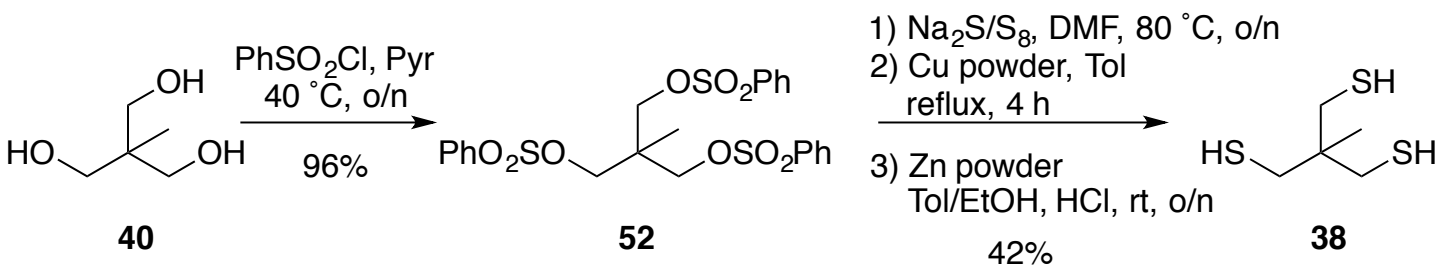

Scheme 38. Successful synthesis of tris(mercaptomethyl)ethane (38). 
Valiulin et al. obtained trithiol $\mathbf{3 8}$ by treating tris(tosyloxymethyl)ethane with a $\mathrm{Na}_{2} \mathrm{~S}_{\mathrm{x}}$ solution in DMF that was prepared by heating a mixture of $\mathrm{NaSH}_{2} 9 \mathrm{H}_{2} \mathrm{O}$ and sulfur powder in DMF to $80{ }^{\circ} \mathrm{C}$ for 1 hour. ${ }^{116}$ The same $\mathrm{Na}_{2} \mathrm{~S}_{\mathrm{x}}$ solution can be obtained by heating a mixture of $\mathrm{Na}_{2} \mathrm{~S}$ and sulfur powder (1:2 molar equivalents, respectively) in DMF to $80{ }^{\circ} \mathrm{C}$ for 1 hour to produce a deep blue solution. ${ }^{118}$ An attempt was thus made to synthesise 38 using Valiulin's procedure, but with substituting $\mathrm{NaSH} 9 \mathrm{H}_{2} \mathrm{O}$ for $\mathrm{Na}_{2} \mathrm{~S}$, as $\mathrm{Na}_{2} \mathrm{~S}$ was immediately at hand.

Accordingly, $\mathrm{Na}_{2} \mathrm{~S}$ and sulfur powder were heated in $\mathrm{DMF}$ at $80{ }^{\circ} \mathrm{C}$ for 1 hour forming a dark blue solution to which benzenesulfonate $\mathbf{5 2}$ was then added, and the reaction left to stir at $80{ }^{\circ} \mathrm{C}$ overnight. The DMF was then removed in vacuo before $\mathrm{Cu}$ powder and toluene were added. The $\mathrm{Cu}$ reduces excess sulfur present and forms black $\mathrm{CuS}$, which was removed via filtration after the mixture was refluxed for an additional 4 hours. The eluent was then diluted with $\mathrm{EtOH}, \mathrm{Zn}$ powder and concentrated $\mathrm{HCl}$ were added, and the solution stirred at room temperature overnight. After extracting the mixture with $\mathrm{CH}_{2} \mathrm{Cl}_{2}$ and purifying the resulting crude oil via silica gel flash chromatography, the desired trithiol 38 was successfully obtained in a $22 \%$ yield, as confirmed by ${ }^{1} \mathrm{H}$ and

${ }^{13} \mathrm{C}$ NMR spectroscopy, which matched the data reported in the literature. ${ }^{116}$ The $\mathrm{CH}_{2}$ proton resonances appear as a doublet at $\delta 2.61\left({ }^{3} J_{H, S}=8.79 \mathrm{~Hz}\right)$, with coupling to the terminal SH protons, while the SH protons appear as a triplet at $\delta 1.22\left({ }^{3} J_{S, H}=8.79 \mathrm{~Hz}\right)$. The methyl protons appear as a singlet $\delta 1.01(\mathrm{~s})$, and the carbon resonances appear at $\delta$ $39.2(\mathrm{Cq}), 31.8\left(\mathrm{CH}_{2}\right)$ and $21.5\left(\mathrm{CH}_{3}\right)$. The yield for the synthesis of $\mathbf{3 8}$ from $\mathbf{5 2}$ was then improved to $42 \%$ by repeating the thiolation reaction, using double the equivalents of $\mathrm{Na}_{2} \mathrm{~S}$ and elemental sulfur (Scheme 38).

\subsubsection{Synthesis of Thiol Functionalised Oxyamine Linker}

With trithiol 38 in hand, the synthesis of the thiol functionalised oxyamine linker was then attempted. Timmer and co-workers synthesised a mono-thiol functionalised oxyamine linker in a $76 \%$ yield over three steps, ${ }^{53}$ whereby 5 equivalents of $1,2-$ propanedithiol relative to acrolein was used. In this manner, propanedithiol simultaneously acts as a reducing agent as well as the nucleophile, and allows for the isolation of the sulfhydryl rather than the disulfide oxyamine. Accordingly, this methodology was initially followed for the synthesis of the di-thiol functionalised 
oxyamine linker 39, where acrolein was reacted with 5 equivalents of neat thiol $\mathbf{3 8}$ at room temperature for 2 hours (Table 2, entry 1). It was observed by TLC that the reaction did not go to completion in this time, however, the synthesis was continued to see if this reaction route was plausible, with optimisation of the individual steps to occur at a later stage. The reaction mixture was then diluted with $\mathrm{EtOH}$, followed by the addition of methoxyamine hydrochloride and sodium acetate, and the solution stirred at room temperature for 18 hours. Reduction of the oxime intermediate was then achieved by adding $\mathrm{NaCNBH}_{3}$ and ethanolic $\mathrm{HCl}$ to the reaction mixture with stirring at room temperature for 5 hours. Purification of the crude mixture by silica gel flash chromatography then led to the successful isolation of linker 39, albeit in a modest (25\%) yield, and in a 5:1 ratio of the dithiol:disulfide products, 39a:39b, respectively. Unreacted trithiol 38 was also recovered (ca. 20\% of the original quantity added).

Table 2. Reaction conditions used for the synthesis of linker 39 using trithiol 38.

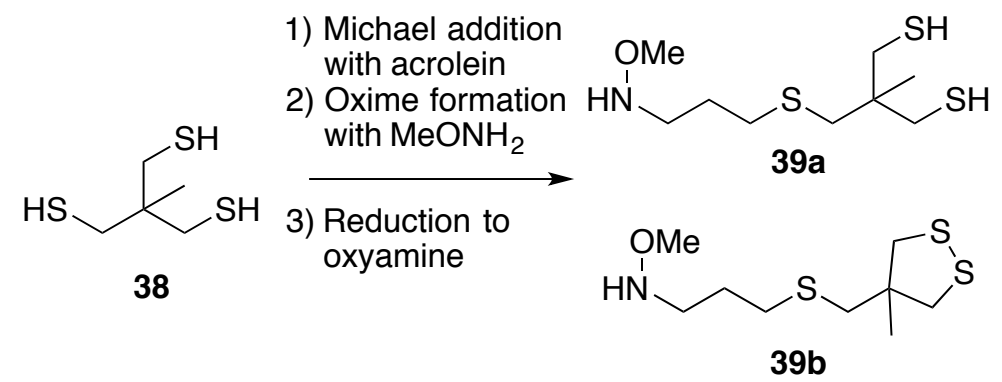

\begin{tabular}{c|c|c|cc|cc|cc}
\hline \multirow{2}{*}{ Entry } & Yield & \multirow{2}{*}{ Eq. } & \multicolumn{2}{|c|}{ Step 1 } & \multicolumn{2}{c|}{ Step 2 } & \multicolumn{2}{c}{ Step 3 } \\
& (39a:39b) & thiol $^{\mathbf{a}}$ & Time & Temp & Time & Temp & Time & Temp \\
\hline 1 & $25 \%(5: 1)$ & 5 & $2 \mathrm{~h}$ & $\mathrm{rt}$ & $18 \mathrm{~h}$ & $\mathrm{rt}$ & $5 \mathrm{~h}$ & $\mathrm{rt}$ \\
2 & $24 \%(1: 1)$ & 1.2 & $\mathrm{o} / \mathrm{n}$ & $\mathrm{rt}$ & $\mathrm{o} / \mathrm{n}$ & $\mathrm{rt}$ & $4 \mathrm{~h}$ & $\mathrm{rt}$ \\
3 & $25 \%(1: 3)$ & 1.1 & $\mathrm{o} / \mathrm{n}$ & $40{ }^{\circ} \mathrm{C}$ & $\mathrm{o} / \mathrm{n}$ & $\mathrm{rt}$ & $5 \mathrm{~h}$ & $\mathrm{rt}$ \\
$4^{\mathrm{b}}$ & $27 \%(3: 1)$ & 1.1 & $\mathrm{o} / \mathrm{n}$ & $40{ }^{\circ} \mathrm{C}$ & $\mathrm{o} / \mathrm{n}$ & $\mathrm{rt}$ & $3 \mathrm{~h}$ & $\mathrm{rt}$ \\
\hline
\end{tabular}

Evidence for the formation of an intramolecular disulfide bond between the two terminal thiols in product 39b was obtained by ${ }^{1} \mathrm{H}$ NMR analysis. Here, the presence of an AB system for the four $\mathrm{CH}_{2}$-S protons was observed: $\delta 3.13\left(\mathrm{~d},{ }^{2} J_{H H}=11.35 \mathrm{~Hz}\right.$ ), 2.89 $\left(\mathrm{d},{ }^{2} J_{H H}=11.35 \mathrm{~Hz}\right)$. This coupling pattern can only be obtained once the disulfide forms, thereby locking the molecule into a rigid 5-membered ring. Given that the reaction conditions appeared to favour formation of intramolecular rather than intermolecular 
disulfide bonds, it was proposed that the 5 equivalents of thiol used in Timmer and coworkers' initial synthesis was unnecessary as dimers or trimers of product 39 appeared less favourable. The reaction was thus repeated using only 1.2 equivalents of thiol (Table 2 , entry 2). The yield was unaffected by the reduced quantity of thiol and the isolated linker was obtained in a 1:1 ratio of ring-closed disulfide to ring-open dithiol. As the trithiol starting material 38 was once again re-isolated (ca. 20\%), this suggested that the reaction of $\mathbf{3 8}$ with acrolein does not go to completion, which was also noted by TLC analysis during the course of the reaction. Accordingly, during the next attempted synthesis of the dithiol oxyamine linker 39, trithiol 38 was heated with acrolein in DCM at $40{ }^{\circ} \mathrm{C}$ overnight (entry 3 ). Unfortunately, this did not improve the reaction yield. The concentration of the reagents for the Michael addition was then increased from $5 \mathrm{~mL} / \mathrm{mmol}$ to $1 \mathrm{~mL} / \mathrm{mmol}$, along with heating at $40{ }^{\circ} \mathrm{C}$ (entry 4 ), however, this also had little effect on reaction yield.

It was then thought that perhaps the steric bulk of trithiol $\mathbf{3 8}$ was interfering with the ability of this nucleophile to partake in the Michael addition to acrolein (step 1). To reduce the effect that steric bulk might have on this reaction, it was proposed that trithiol $\mathbf{3 8}$ could be oxidised to form an intramolecular disulfide bond as this would tether two of the thiol groups in a 5-membered ring and potentially render the remaining thiol more reactive.

The oxidation of $\mathbf{3 8}$ to dithiolane $\mathbf{5 3}$ was first attempted using one equivalent of iodine (Table 3, entry 1). Trithiol 38 was dissolved in $\mathrm{CH}_{2} \mathrm{Cl}_{2}$ before the addition of iodine and water, whereby water helps dissolve the iodine and is required to dissolve the HI formed during the reaction. This mixture was vigorously stirred for one hour, however, only the over-oxidised dimer 54 was observed, as evidenced by the ${ }^{1} \mathrm{H}$ NMR spectrum where there were no observable SH proton resonances or ${ }^{1} \mathrm{H}-{ }^{1} \mathrm{H}$ coupling between the $\mathrm{CH}_{2}$ protons and the SH proton, both of which are quite evident in the ${ }^{1} \mathrm{H}$ NMR spectrum of $\mathbf{3 8}$. The $\mathrm{AB}$ system of the 5-membered disulfide ring in $\mathbf{5 4}$ moved downfield to $\delta 3.13$ and $\delta 2.90$, while the other $\mathrm{CH}_{2}$ protons also appeared downfield $(\delta 3.08, \mathrm{~s})$ relative to the $\mathrm{CH}_{2}-\mathrm{SH}$ protons of 38 which appear at $\delta 2.61\left(\mathrm{~d}, J_{\mathrm{SH}, \mathrm{CH} 2}=8.8 \mathrm{~Hz}\right)$. This suggests that the $\mathrm{CH}_{2}$ protons not involved in the 5-membered ring are also adjacent to a disulfide bond rather than to a thiol. The oxidation was attempted again using one equivalent of iodine that was 
added as a $0.1 \mathrm{M}$ aqueous solution. Again, only $\mathbf{5 4}$ was formed (entry 2). It was then thought that diluting the reaction solution might prevent dimer formation. Accordingly, the concentration of the solution was thus diluted to $20 \mathrm{~mL} / \mathrm{mmol}$, from the original $5 \mathrm{~mL} / \mathrm{mmol}$, and only 0.1 equivalents of iodine was added to assess if any of the desired product 53 formed before dimer 54. Under these reaction conditions, a mixture of $\mathbf{5 3}$, starting material 38, and dimer 54 were observed by ${ }^{1} \mathrm{H}$ NMR spectroscopy in a 1:3:1 ratio, respectively (entry 3), which suggested that there was scope to optimise this reaction. The formation of $\mathbf{5 3}$ was confirmed by ${ }^{1} \mathrm{H}$ NMR spectroscopy by the presence of a doublet $\left({ }^{3} J_{H, S H}=10.5 \mathrm{~Hz}\right)$ at $\delta 2.72$ belonging to the $\mathrm{CH}_{2}-\mathrm{SH}$ protons, which had moved downfield relative to the $\mathrm{CH}_{2}-\mathrm{SH}$ protons of starting material 38. The equivalents of iodine added was then increased to 0.8 in an attempt to favour the formation of $\mathbf{5 3}$. Indeed, the relative amount of the desired product improved (3:1:2, 53:38:54), with 53 and 38 being isolated in a 74\% combined yield (entry 4). These findings suggested that as more of the target dithiolane $\mathbf{5 3}$ is produced, the amount of dimer $\mathbf{5 4}$ also increases before all starting material has reacted. Nonetheless, another reaction was attempted by adding iodine in $10 \times 0.1$ equivalent aliquots. It was hoped that the slow addition of the iodine under these dilute conditions would favour the production of dithiolane $\mathbf{5 3}$ relative to the dimer. Indeed, these conditions gave 53:38:54 in a 5:1:2 ratio (79\% combined yield) (entry 5). The addition of more than one equivalent of iodine, however, did not further improve this ratio and instead resulted in the production of more dimer (entry 6). Silica gel flash chromatography of the 5:1:2 mixture of 53:38:54 resulted in the separation of dimer 54 from dithiolane 53 and dithiol 38. Unfortunately, 53 and $\mathbf{3 8}$ were inseparable using silica gel flash chromatography as they had the same $\mathrm{R}_{f}$ by $\mathrm{TLC}\left(\mathrm{R}_{f}=0.79\right.$ $(\mathrm{PE} / \mathrm{EtOAc}, 3: 1, \mathrm{v} / \mathrm{v}))$. A further attempt was made to favour the production of $\mathbf{5 3}$ by using the same reaction conditions as entry 5 , but at $-20{ }^{\circ} \mathrm{C}$, however, the ratio of products did not improve. 
Table 3. Reaction conditions used for the synthesis of disulfide $\mathbf{5 3}$ from trithiol $\mathbf{3 8}$.

\begin{tabular}{|c|c|c|c|}
\hline & & $\sum_{38}^{\mathrm{SH}} \mathrm{SH}$ & $H S \underbrace{S}_{53}$ \\
\hline Entry & Eq. $I_{2}$ & $\begin{array}{c}\text { Reaction } \\
\text { Conc. } \\
\text { (mL/mmol) }\end{array}$ & $\begin{array}{c}\text { Observed } \\
\text { (yield, includes } 53 \text { and 38) }\end{array}$ \\
\hline 1 & 1 & 5 & $\begin{array}{ll}2 & 54\end{array}$ \\
\hline $2^{\mathrm{a}}$ & 1 & 5 & $2^{+S}$ \\
\hline $3^{\mathrm{a}}$ & 0.1 & 20 & $\begin{array}{c}38 \\
1: 3: 1(72 \%)\end{array}$ \\
\hline $4^{\mathrm{a}}$ & 0.8 & 20 & $\begin{array}{c}38 \\
3: 1: 2(74 \%)\end{array}$ \\
\hline $5^{\mathrm{a}, \mathrm{b}}$ & 1 & 20 & $\begin{array}{c}38 \\
5: 1: 2(79 \%)\end{array}$ \\
\hline 6 & 1.4 & 20 & $\begin{array}{c}38 \\
5.5: 1: 3(55 \%)\end{array}$ \\
\hline
\end{tabular}

${ }^{\mathrm{a}} \mathrm{I}_{2}$ added as a $0.1 \mathrm{M}$ aqueous solution; ${ }^{\mathrm{b}} \mathrm{I}_{2}$ solution added 0.1 equivalents at a time

In an attempt to determine whether the addition of the thiol linker as a dithiolane would improve the reaction yield for the subsequent formation of the target oxyamine linker 39, the linker synthesis was then attempted using the 5:1 mixture of 53:38. Unfortunately, this produced no significant increase in the yield, with the oxyamine linker 39 being 
obtained in a $26 \%$ yield, exclusively as the disulfide $39 \mathrm{~b}$, following purification by silica gel flash column chromatography (Scheme 39).

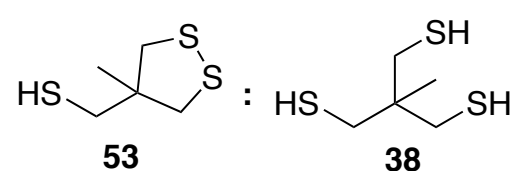

$(5: 1)$

1) Acrolein, $\mathrm{CH}_{2} \mathrm{Cl}_{2}$, rt, o/n

2) $\mathrm{MeONH}_{2} \mathrm{HCl}, \mathrm{NaOAC}$ $\mathrm{EtOH}, \mathrm{rt}, \mathrm{o} / \mathrm{n}$

3) $\mathrm{NaCNBH}_{3}, \mathrm{EtOH}$ $\mathrm{HCl}$, rt, $4 \mathrm{~h}$

$26 \%$

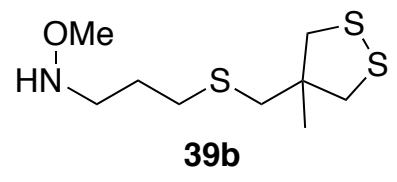

$39 b$

Scheme 39. Synthesis of oxyamine linker 39b using a 5:1 mixture of dithiolane 53:dithiol 38 .

Given these results, the reaction conditions presented in entry 4 of Table 2 were thus used to prepare more of the target linker (39a:39b; 3:1, 27\% yield). During the linker synthesis however, a by-product was isolated from the crude reaction mixture following purification by silica gel flash chromatography and was revealed to be acetal $\mathbf{5 5}$ (Figure 28), identified by $1 \mathrm{D}$ and $2 \mathrm{D}$ NMR spectroscopy $\left(\mathrm{CDCl}_{3}\right)$. Here, the $\mathrm{CH}(\mathrm{OEt})_{2}$ proton appears as a triplet at $\delta 4.60\left({ }^{3} J_{H, H}=5.7 \mathrm{~Hz}\right)$ and the $\mathrm{CH}_{2}$ protons belonging to the $\mathrm{OEt}$ groups form an $\mathrm{AB}$ system as two characteristic quintets at $\delta 3.65$ and $\delta 3.50\left(J_{H, H}=7.5\right.$ $\mathrm{Hz}$ ). The high chemical shift of these protons is consistent with protons adjacent to the oxygen atoms in the acetal functional group. The methyls belonging to the OEt groups appear as a triplet at $\delta 1.21\left({ }^{3} J_{H, H}=7.4 \mathrm{~Hz}\right)$. In some instances, 55 formed up to a 1:1 mixture with the desired product 39. It was thus proposed that the formation of $\mathbf{5 5}$ accounted for the low yield of the reaction, which suggests that either the reaction of the intermediate aldehyde with methoxyamine or the reduction of the oxime formed in the second step of the reaction sequence have not gone to completion. Accordingly, $\mathbf{3 8}$ was reacted with acrolein with stirring at room temperature for two days, followed by dilution with EtOH and the simultaneous addition of methoxyamine hydrochloride, $\mathrm{NaOAc}$ and $\mathrm{NaCNBH}_{3}$. The mixture was left to stir at room temperature for three days. Adding the reducing agent at the same time as methoxyamine should not affect the aldehyde as $\mathrm{NaCNBH}_{3}$ selectively reduces imines. TLC analysis at the end of the three day period indicated that linker 39 had formed $\left(\mathrm{R}_{f}=0.33(\mathrm{PE} / \mathrm{EtOAc}, 3: 1, \mathrm{v} / \mathrm{v})\right)$ with the absence of any acetal by-product $\left(\mathrm{R}_{f}=0.40(\mathrm{PE} / \mathrm{EtOAc}, 3: 1, \mathrm{v} / \mathrm{v})\right)$, however, there was still a substantial quantity of unreacted thiol $\left(\mathrm{R}_{f}=0.79(\mathrm{PE} / \mathrm{EtOAc}, 3: 1, \mathrm{v} / \mathrm{v})\right)$. Nonetheless, the mixture was worked up to assess if the absence of by-product 55 would increase the yield. 
A ${ }^{1} \mathrm{H}$ NMR spectrum of the crude mixture confirmed the absence of $\mathbf{5 5}$. Unfortunately, following purification by silica gel flash chromatography, linker 39 was only isolated in a $25 \%$ yield. While these results have presented an effective method for the prevention of the formation of by-product 55, it also indicates that the Michael addition of the thiol to acrolein needs optimising and is the likely cause of the low yield for the linker synthesis.

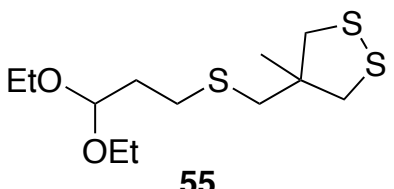

Figure 28. By-product formed during the synthesis of linker 39.

\subsubsection{Glycosylation Reactions using the Trithiol Functionalised Oxyamine Linker}

With the thiol functionalised oxyamine linker 39 successfully synthesised, it was then proposed that this would be conjugated to a simple monosaccharide as a proof of concept for subsequent glycosylation studies. To this end, GlcNAc was the monomer of choice as it is a simple and inexpensive monosaccharide, and is also the monomer unit of chitin. The GlcNAc glycoconjugate can also be attached to AuNPs and tested in biological assays along with other chitin functionalised AuNPs, which will be prepared at a later date.

Timmer and co-workers conjugated their thiol functionalised oxyamine linker to GlcNAc in a solvent mixture of 2:2:1 NaOAc/AcOH buffer (2 M, pH 4.5): $\mathrm{EtOH}: \mathrm{CH}_{2} \mathrm{Cl}_{2}$ at $40{ }^{\circ} \mathrm{C}$ for 72 hours. ${ }^{53}$ The decreased polarity of the thiol functionalised linkers compared to the analogous amine functionalised oxyamine linkers means that the glycosylation reactions with the thiol linkers cannot be performed in acetate buffer alone as the linker would be insoluble. Timmer and co-workers' method was thus followed for the first attempt at the conjugation of linker 39 to GlcNAc. Here, linker 39 was dissolved in the 2:2:1 solvent mixture before 2 equivalents of GlcNAc were added so that the concentration of the reaction was approximately $1 \mathrm{~mL} / \mathrm{mmol}$. The mixture was then stirred at $40{ }^{\circ} \mathrm{C}$ for 72 hours. Unfortunately, GlcNAc did not fully dissolve under these reaction conditions and produced a milky coloured solution. Nonetheless, the reaction mixture was purified by size exclusion chromatography to produce glycoside $\mathbf{5 6}$ in a low 7.5\% yield (Scheme 40). 
Evidence for the formation of the target glycoconjugate $\mathbf{5 6}$ includes the presence of a single anomeric proton resonance at $\delta 4.31$ for the $\beta$-pyranoside $\left({ }^{3} J_{1,2}=9.7 \mathrm{~Hz}\right)$, which has moved upfield compared to the uncoupled sugar at $\delta 5.17\left(\mathrm{~d},{ }^{3} J_{1,2}=3.4 \mathrm{~Hz}, \alpha\right.$ GlcNAc) and $\delta 4.68\left(\mathrm{~d},{ }^{3} J_{1,2}=8.3 \mathrm{~Hz}, \beta\right.$-GlcNAc) (Figure 29). The conjugation of linker 39 to GlcNAc was also confirmed by the presence of an $\mathrm{HMBC}$ between the $\mathrm{N}-\mathrm{CH}_{2}$ and anomeric resonances (Figure 30). HRMS also confirmed the presence of the glycoconjugate $\left(m / z\right.$ calcd. for $\left[\mathrm{C}_{17} \mathrm{H}_{32} \mathrm{~N}_{2} \mathrm{O}_{6} \mathrm{~S}_{3} \mathrm{~K}\right]^{+}: 479.1315$, obsd.:479.1337).

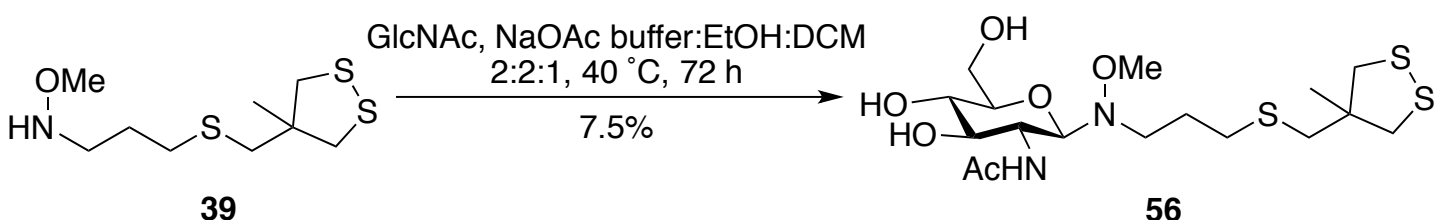

Scheme 40. Synthesis of glycoconjugate 56.

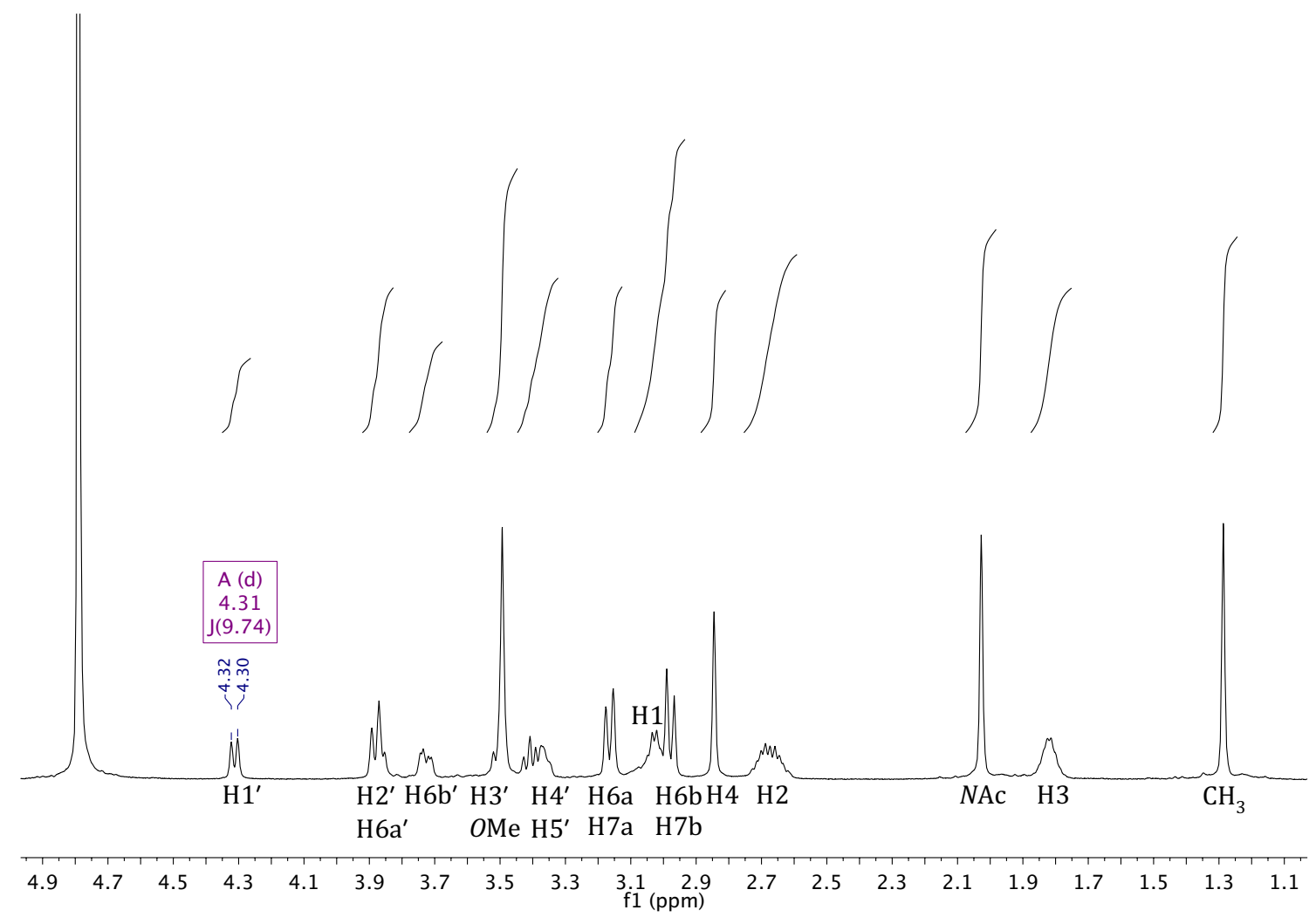

Figure 29. ${ }^{1} \mathrm{H}$ NMR spectrum for glycoside $56\left(\mathrm{D}_{2} \mathrm{O}, 500 \mathrm{MHz}\right)$. 


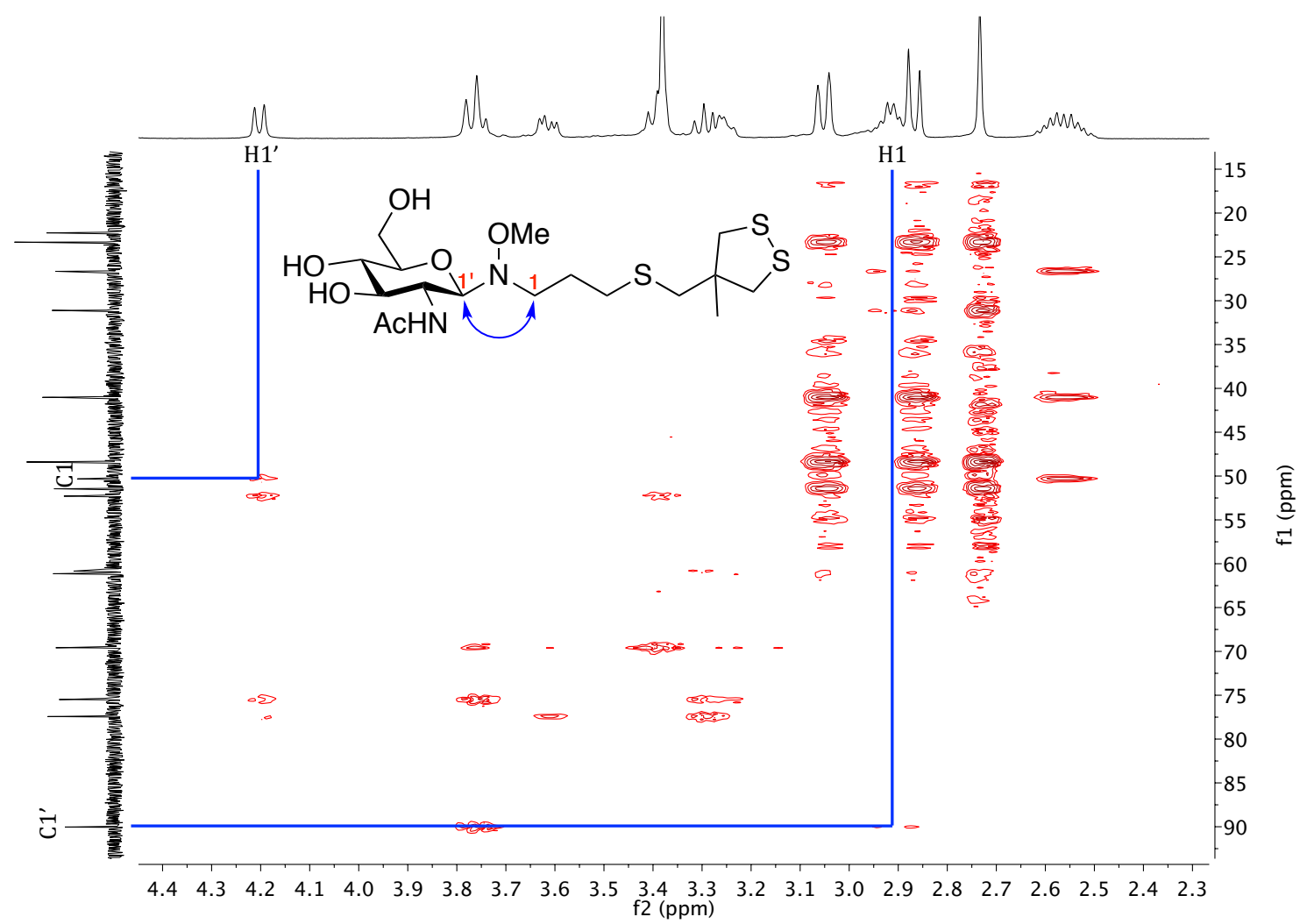

Figure 30. $\mathrm{HMBC}$ for conjugate $56\left(\mathrm{D}_{2} \mathrm{O}, 500 \mathrm{MHz}\right)$.

In an attempt to increase the yield of the glycosylation, the reaction was repeated using pyridine as the solvent with one equivalent of $\mathrm{AcOH}$. While linker 39 dissolved in this solvent mixture, GlcNAc was not entirely soluble at this high concentration ( $1 \mathrm{~mL} / \mathrm{mmol})$. Unfortunately, no product was isolated. The glycosylation was then attempted using a 1:1 mixture of DMSO:AcOH/NaOAc buffer ( $\mathrm{pH} 4.5,2 \mathrm{M}$ ). Both 39 and GlcNAc are soluble in this mixture upon heating to $40{ }^{\circ} \mathrm{C}$. The mixture was stirred at $40{ }^{\circ} \mathrm{C}$ for three days. TLC analysis revealed the disappearance of linker 39 and the formation of a product which ran higher than GlcNAc $\left(\mathrm{R}_{f}=0.63\left(\mathrm{MeOH} / \mathrm{CH}_{2} \mathrm{Cl}_{2}, 1: 4\right.\right.$, $\mathrm{v} / \mathrm{v})$ ), thought to be the acetate salt of the desired glycoside. The crude mixture was purified by size exclusion chromatography to provide glycoside 56 in a much increased $49 \%$ yield (Scheme 41 ).

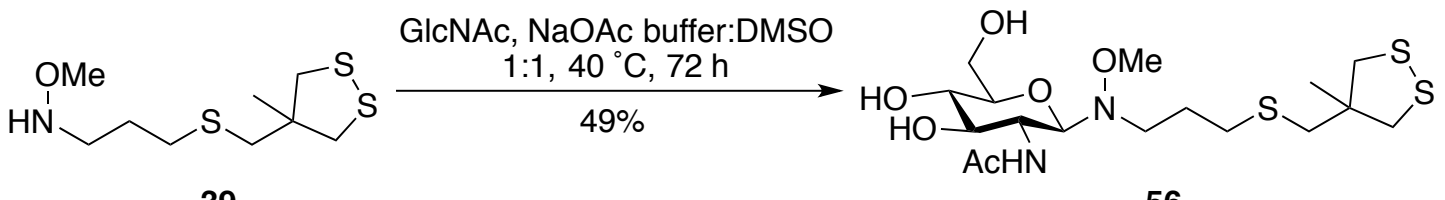

39 56

Scheme 41. Improved synthesis of glycoconjugate 56. 
The successful synthesis of glycoside $\mathbf{5 6}$ in a moderate yield illustrates the proof of concept for the synthesis of dithiol-functionalised glycosyloxyamines. In particular, the merit of this methodology for attaching chitin fragments of various sizes has been demonstrated. It is anticipated that the conjugation of longer chitin fragments should not present too many challenges as the identity of the sugar at its free reducing end is the same (GlcNAc), and the polysaccharides are linear ( $\beta$-1,4-linkages). Their increased steric bulk should therefore not interfere with the chemistry at the free reducing end. It is also envisioned that these longer fragments will have similar solubility to the GlcNAc monosaccharide, however, optimisation of the solvent conditions for the glycan conjugation may still need to occur.

Once these glycoconjugates have been synthesised with varying lengths of chitin, the conjugates can be attached to AuNPs of various sizes to mimic chitin particles. The presence of the 5-membered disulfide ring instead of the two free thiols on the conjugates will not hinder attachment to the AuNP surface as these disulfide bonds are reduced during the synthesis of the NPs and adhere as the free thiols. ${ }^{104}$ The effect of chitin particle size on the immune response by macrophages and DCs can then be explored.

\subsection{Conclusion}

A novel dithiol functionalised oxyamine linker was successfully synthesised and conjugated to GlcNAc, the monomer unit of chitin. This bifunctional oxyamine linker was synthesised by adapting Timmer and co-workers' method for the synthesis of "Type B" oxyamine linkers, thereby demonstrating the adaptability of this route for the synthesis of linkers that vary in their terminal functional groups.

This linker harbours great potential for the multivalent display of glycans on AuNPs. The linker is easily synthesised in 5-steps and is conjugated to the free reducing end of a saccharide in one step, without the need for any protecting groups, thereby enabling the use of both synthetically derived and biologically derived saccharides. It is envisioned that the bidentate attachment of dithiol-functionalised glycans to AuNPs will facilitate in the stability of such glycoconjugates and make them versatile reagents for biological 
applications, including investigations into the effect of chitin size on the allergic immune response. 


\section{Chapter 5}

\section{Conclusion and Future Prospects}

\subsection{Conclusion}

This Master's thesis has focused on the development of bi-functional $N, O$ dialkyloxyamine linkers for the synthesis of glycoconjugates. The merits of "Type A" and "Type B" glycosyloxyamines have been directly compared for the first time. During this study, the synthesis of a "Type A" linker containing an amine at its terminus was improved by reducing the number of synthetic steps from $\operatorname{six}^{51}$ to four and by increasing the overall yield from $8 \%$ to $38 \%$. The corresponding "Type A" and "Type B" glycoconjugates were then directly compared regarding the effect of $\mathrm{pH}$, glycoside concentration and buffer strength on their hydrolytic stability. The "Type B" glycoside was found to have marginally better kinetic stability, while the "Type A" glycoside had slightly better thermodynamic stability across all the variables tested. However, these differences are modest and are likely to have little effect in practice.

To gain a better understanding of the hydrolytic stability of these glycosyloxyamines, the $\mathrm{p} K_{\mathrm{a}}$ of the conjugate acid of a generic glycosyloxyamine was successfully determined to be -0.9 using ${ }^{1} \mathrm{H}$ NMR titration experiments. This value corresponds to the $N$-protonated form of the glycoside and indicates that the anomeric nitrogen is the most basic site on the conjugate. It was thus inferred that the ring oxygen is less basic and therefore, once protonated, will have a $\mathrm{p} K_{\mathrm{a}}$ lower than -0.9 . This indicates that the ring oxygen requires a very low $\mathrm{pH}$ to become protonated, which explains the high stability of the glycoconjugates under basic, neutral and mildly acidic conditions. The studies herein also suggest that the hydrolysis of these conjugates is a GAC process between $\mathrm{pH} 4-6$, which is contrary to the previous literature that states that it is a SAC process. ${ }^{38,43,50,66}$

Notwithstanding the improvement to the synthesis of the amine-functionalised "Type A" oxyamine linker, the "Type B" linker synthesis is still more concise and adaptable for the production of linkers with different terminal functional groups. ${ }^{53,89}$ Accordingly, a novel dithiol functionalised oxyamine linker was designed, synthesised, and conjugated to 
GlcNAc, the monomer unit of chitin, by adapting Timmer and co-workers' methodology for the synthesis of "Type B" oxyamine linkers. Not only does this demonstrate the versatility of the "Type B" route for the synthesis of oxyamine linkers that vary in their terminal functional groups, but moreover, the bidentate nature of this new thiol linker means it will be better able to coordinate to, and form stable bonds with AuNPs. The application of this linker for the conjugation of chitin fragments to AuNPs has been demonstrated via the conjugation of the chitin building block, GlcNAc, to the linker. It is envisioned that larger chitin fragments can also be conjugated to the linker using a similar methodology and that these oxyamine-linked glycans can then be coupled to AuNPs using standard literature protocols.

\subsection{Future Prospects}

The studies regarding the hydrolytic stability of the "Type A" and "Type B" glycosyloxyamines have clearly demonstrated the conditions in which these conjugates are stable. Determining the $\mathrm{p} K_{\mathrm{a}}$ of the conjugate acid of a generic glycosyloxyamine has provided a better understanding of the mechanism of hydrolysis of these conjugates, however, further studies into the mechanism of the hydrolysis of these conjugates would be valuable. The work presented herein has suggested that hydrolysis occurs via GAC at $\mathrm{pH} 4-6$, which is contrary to the literature which states that SAC occurs in this $\mathrm{pH}$ range. The studies herein have also suggested that the type of catalysis occurring, and therefore the RDS, may also change with $\mathrm{pH}$. Thus, to better understand the mechanism of glycosyloxyamine hydrolysis, the hydrolysis studies need to be performed using a much wider $\mathrm{pH}$ range and specifically include studies under highly acidic conditions. This will indicate whether the RDS varies with $\mathrm{pH}$, and therefore give a better indication as to the type of catalysis that is occurring at various $\mathrm{pH}$ values. A Hammett plot could also be constructed to determine the nature of the transition state during the RDS. ${ }^{87}$ The $\rho$ value (Hammett reaction constant) obtained from a Hammett plot will indicate whether a positive or negative charge is being developed during the TS, and thus aid in distinguishing between the SAC and GAC mechanisms. A Hammett plot can be obtained by synthesising glycosyloxyamines containing $N$-phenyl oxyamines with electron donating or withdrawing substituents at the para position, and plotting the rate of hydrolysis for the functionalised glycosyloxyamines compared to the un-functionalised 
glycosyloxyamine $\left(\log k_{\mathrm{X}} / k_{\mathrm{H}}\right)$ against the Hammett substituent constant $\sigma$ (electron withdrawing/donating character). A positive $\rho$ value, as determined by the slope of the Hammett plot, indicates that electron withdrawing groups increase the rate of hydrolysis by stabilising the TS and therefore suggests that a negative charge is developing during the TS, as would be observed if GBC was the RDS (with SAC occurring prior to the RDS). Conversely, a negative $\rho$ value indicates that a positive charge is developing during the TS, as would be observed if GAC was the RDS. Any breaks in a continuous trend on the Hammett plot will also indicate that a change in the RDS is occurring due to the different substituents. ${ }^{87}$ Several Hammett plots can also be constructed at different $\mathrm{pH}$ values, which can determine if a change in the RDS occurs due to $\mathrm{pH} .{ }^{87}$ Kinetic solvent isotope studies could also be conducted to differentiate between SAC and GAC. As mentioned in Chapter 3 of this thesis, SAC involves an inverse solvent isotope effect $\left(k\left[\mathrm{D}_{2} \mathrm{O}\right]>k\left[\mathrm{H}_{2} \mathrm{O}\right]\right)$. The presence of SAC can therefore be determined by conducting more hydrolysis studies and noting any effect on the rate caused by deuterated $v s$. nondeuterated solvents. As discussed in Chapter 3, this study can also be conducted using polarimetry by noting how quickly the optical rotation reduces to zero in the different solvents as the glycoconjugates hydrolyse.

The successful attachment of the monomeric unit of chitin to the novel dithiol functionalised oxyamine linker has laid the foundations for the future production of chitin functionalised AuNPs. While the synthesis of the disulfide oxyamine linker has been synthesised in a reasonable (27\%) yield, this reaction could be improved by further optimising the Michael addition of the trithiol to acrolein. Similarly, the glycosylation reaction with the linker and GlcNAc has demonstrated proof-of-principle to give the glycoconjugates in a moderate $49 \%$ yield, however, this could be further improved by investigating more solvent systems for the reaction. The glycosylation reaction needs to be further optimised before the attachment of the longer chitin oligomers to this oxyamine linker. The chitin glycosyloxyamines can be conjugated to AuNPs of varying sizes, which can then be used to test the effect that chitin particle size and chain length has on eliciting an allergic immune response. First, cell-based assays will be used. The phagocytosis of chitin-coated AuNPs by macrophages can be studied and the type of immune response (i.e. cytokine production and changes to cell surface markers) detected by ELISA and flow cytometry. Similarly, T-cell assays, involving the co-administration of the chitin- 
functionalised AuNPs with and without the model antigen (chitin) and OVA will be undertaken to assess allergic immune responses. Following these studies, more advanced in vivo assays could also be undertaken at a later date. ${ }^{119}$ 


\section{Chapter 6}

\section{Experimental}

\subsection{General Procedure}

Unless otherwise stated all reactions were performed under atmospheric air. Methanol (MeOH, Pure Science), ethanol (EtOH, absolute, Pure Science), dichloromethane $\left(\mathrm{CH}_{2} \mathrm{Cl}_{2}\right.$, LabServ), carbon tetrachloride (AnalaR), and toluene (ROTH) were used as received. $\mathrm{H}_{2} \mathrm{O}$, ethyl acetate (EtOAc, from drum), petroleum ether (PE, from drum), $\mathrm{Et}_{3} \mathrm{~N}$ (Sigma), DMF (Acros), pyridine (Romal) and THF (Chemsolute) were distilled prior to use. Bromoethylamine hydrobromide (Aldrich), $\operatorname{Boc}_{2} \mathrm{O} \quad$ (Sigma), N,Odimethylhydroxylamine hydrochloride (Aldrich), NaH (Aldrich), TFA (Aldrich), acrolein (Merck), AcOH (Ajax Finechem), $\mathrm{NaN}_{3}(\mathrm{BDH}), 30 \%$ aqueous $\mathrm{NH}_{3}$ (J. T. Baker Chemical Co.), $\mathrm{AcCl}$ (Sigma Aldrich), $\mathrm{NaCNBH}_{3}$ (Aldrich), $\mathrm{NaHCO}_{3}$ (Pure Science), $\mathrm{MgSO}_{4}$ (Pure Science), $\mathrm{NH}_{4} \mathrm{OAc}$ (Sigma Aldrich), methoxyamine hydrochloride (Sigma Aldrich), $\mathrm{N}$-acetylglucosamine (Fluka), dansyl chloride (Applichem), $\mathrm{HCl}$ (concentrated, Univar), $\mathrm{NH}_{4} \mathrm{HCO}_{2}$ (AnalaR), $t$-BuOH (Unilab), DCl (concentrated, Aldrich), NaOD (40\% wt, Aldrich), $\mathrm{CDCl}_{3}$ (Aldrich), $\mathrm{D}_{2} \mathrm{O}$ (Apollo), tris(hydroxymethyl)ethane (Aldrich), benzenesulfonate $(\mathrm{BDH})$, sulfur powder (Pharmaceutical Sales and Marketing Ltd.), $\mathrm{Na}_{2} \mathrm{~S}$ (Pure Science), copper powder (J. T. Baker), zinc powder (BDH), $\mathrm{PBr}_{3}$ (Aldrich), dibromobutane (Aldrich), thiourea (Unilab), allyl bromide (Aldrich), $\mathrm{Br}_{2}$ (Sigma Aldrich) and mercaptoethanol (Aldrich) were used as received. All solvents were removed by evaporation under reduced pressure. Reactions were monitored by TLC-analysis on silica gel coated plastic sheets $(0.20 \mathrm{~mm}$, silicagel 60) with detection by UV-absorption (254 nm), by dipping in $10 \% \mathrm{H}_{2} \mathrm{SO}_{4}$ in $\mathrm{EtOH}$ followed by charring at $\sim 150{ }^{\circ} \mathrm{C}$, by dipping in $\mathrm{I}_{2}$ in silica, by dipping in a solution of ninhydrin in EtOH followed by charring at $\sim 150{ }^{\circ} \mathrm{C}$ or by dipping in a solution of $5 \% \mathrm{~K}_{2} \mathrm{CO}_{3}, 1 \% \mathrm{KMnO}_{4}$ and $1 \% \mathrm{NaIO}_{4}$ in $\mathrm{H}_{2} \mathrm{O}$. Column chromatography was performed on Pure Science silica gel (40-63 micron). AccuBOND II ODS-C18 (Agilent) was used for reverse phase chromatography. Bio-Gel P-2 $(600 \times 10 \mathrm{~mm})$ was used for size exclusion chromatography. Optical rotations were recorded using a Perkin-Elmer 241 polarimeter at the sodium D-line. Infrared spectra were recorded as thin films using a Bruker Tensor 27 FTIR spectrometer equipped with 
an Attenuated Total Reflectance (ATR) sampling accessory and are reported in wave numbers $\left(\mathrm{cm}^{-1}\right)$. High-resolution mass spectra were recorded on an Agilent Technology 6530 Accurate Mass Q-TOF/LCMS spectrometer using positive electro-spray ionisation. Nuclear magnetic resonance spectra were recorded at $20^{\circ} \mathrm{C}$ in $\mathrm{D}_{2} \mathrm{O}$ or $\mathrm{CDCl}_{3}$ using either a Varian Unity-INOVA operating at $300 \mathrm{MHz}$ or a Varian Unity operating at $500 \mathrm{MHz}$. Chemical shifts are given in ppm $(\delta)$ relative to tetramethylsilane. NMR peak assignments were made using COSY, HSQC and HMBC 2D experiments.

\subsection{Chemical Synthesis}

Boc $\mathrm{N}_{\mathrm{H}} \mathrm{Br}$ 2-(N-Boc-amino)ethyl bromide (2). To a solution of bromoethylamine hydrobromide $(2.1 \mathrm{~g}, 10.3 \mathrm{mmol})$ and $\mathrm{Boc}_{2} \mathrm{O}(2.3 \mathrm{~mL}, 10 \mathrm{mmol})$ in freshly distilled $\mathrm{CH}_{2} \mathrm{Cl}_{2}(7 \mathrm{~mL}), \mathrm{Et}_{3} \mathrm{~N}(1.4 \mathrm{~mL}, 10.3 \mathrm{mmol})$ was added and the reaction mixture then stirred at $\mathrm{rt}$ for $4 \mathrm{~h}$ under argon. The reaction was then quenched with $0.1 \mathrm{M}$ $\mathrm{HCl}(10 \mathrm{~mL})$ and extracted with EtOAc $(2 \times 20 \mathrm{~mL})$. The organic layers were combined and washed with a sat. aq. $\mathrm{NaHCO}_{3}$ and brine before being dried over $\mathrm{MgSO}_{4}$. The solution was filtered and the solvent removed in vacuo to give the title compound as a colourless oil, which was used without further purification $(2.1 \mathrm{~g}, 89 \%) . \mathrm{R}_{f}=0.38$ (PE/EtOAc, 9/1, v/v); IR (film) 3339, 2977, 2932, 1689, 1509, $1164 \mathrm{~cm}^{-1} ;{ }^{1} \mathrm{H}$ NMR (500 $\left.\mathrm{MHz} \mathrm{CDCl}_{3}\right) \delta 5.05(\mathrm{bs}, 1 \mathrm{H}, \mathrm{NH}), 3.50\left(\mathrm{dt}, 2 \mathrm{H}, J_{1,2}=5.4 \mathrm{~Hz}, J_{2, N H}=5.6 \mathrm{~Hz}, \mathrm{CH}_{2} \mathrm{Br}\right)$, $3.42\left(\mathrm{t}, 2 \mathrm{H}, J_{1,2}=5.4 \mathrm{~Hz}, \mathrm{CH}_{2} \mathrm{~N}\right), 1.41\left(\mathrm{~s}, 9 \mathrm{H}, \mathrm{CH}_{3} t \mathrm{Bu}\right) ;{ }^{13} \mathrm{C} \mathrm{NMR}\left(125 \mathrm{MHz}, \mathrm{CDCl}_{3}\right)$ $\delta 155.7(\mathrm{C}=\mathrm{O}), 79.8(\mathrm{Cq} t \mathrm{Bu}), 42.4\left(\mathrm{CH}_{2} \mathrm{Br}\right), 32.6\left(\mathrm{CH}_{2} \mathrm{~N}\right) ; 28.4\left(\mathrm{CH}_{3} t \mathrm{Bu}\right) ; \mathrm{HRMS}(\mathrm{ESI})$ $\mathrm{m} / \mathrm{z}$ calcd. for $\mathrm{M}-\mathrm{Boc}+\mathrm{H}^{+}\left[\mathrm{C}_{2} \mathrm{H}_{7} \mathrm{BrN}\right]^{+}:$123.9756, obsd.: 123.9778 .

$\begin{array}{cl}\mathrm{HO}_{\substack{N^{\prime} \\ \text { Boc }}} & \boldsymbol{N} \text {-Boc- } \boldsymbol{N} \text {-methylhydroxylamine } \\ \text { methylhydroxylamine hydrochloride }(0.84 \mathrm{~g}, 10 \mathrm{mmol}) \text { and } \mathrm{Boc}_{2} \mathrm{O}(2.5 \mathrm{~mL} \text {, }\end{array}$ $11 \mathrm{mmol})$ in freshly distilled $\mathrm{CH}_{2} \mathrm{Cl}_{2}(7 \mathrm{~mL}), \mathrm{Et}_{3} \mathrm{~N}$ was added $(1.5 \mathrm{~mL}, 11 \mathrm{mmol})$ and the resulting reaction mixture was stirred at $\mathrm{rt}$ for $4 \mathrm{~h}$ under argon. The reaction mixture was quenched with $0.1 \mathrm{M} \mathrm{HCl}(10 \mathrm{~mL})$ and extracted with EtOAc $(2 \times 20 \mathrm{~mL})$. The combined organic layers were then washed with sat. aq. $\mathrm{NaHCO}_{3}$ and brine before being dried over $\mathrm{MgSO}_{4}$ and filtered. The solvent was removed under reduced pressure to afford hydroxylamine 4 as an oil, which was used without further purification (1.05 g, 7.1 mmol, $71 \%) . \mathrm{R}_{f}=0.14(\mathrm{PE} /$ EtOAc, 9/1, v/v); IR (film) 3255, 2977, 2930, 1688, 1477, 1365, 
1252, 1147, 1023, 848, $760 \mathrm{~cm}^{-1} ;{ }^{1} \mathrm{H}$ NMR (500 MHz, $\left.\mathrm{CDCl}_{3}\right) \delta 7.39$ (bs, $\left.1 \mathrm{H}, \mathrm{OH}\right), \delta 3.15$ $\left(\mathrm{s}, 3 \mathrm{H}, \mathrm{N}-\mathrm{CH}_{3}\right), \delta 1.47\left(\mathrm{~s}, 9 \mathrm{H}, \mathrm{CH}_{3} t \mathrm{Bu}\right) ;{ }^{13} \mathrm{C} \mathrm{NMR}\left(125 \mathrm{MHz}, \mathrm{CDCl}_{3}\right) \delta 157.8(\mathrm{C}=\mathrm{O})$, $82.0(\mathrm{Cq}), 38.0\left(\mathrm{~N}-\mathrm{CH}_{3}\right), 28.4\left(\mathrm{CH}_{3} t \mathrm{Bu}\right)$; HRMS(ESI) m/z calcd. for $\left[\mathrm{C}_{6} \mathrm{H}_{14} \mathrm{NO}_{3}\right]^{+}$: 148.0968, obsd.: 148.0975 .

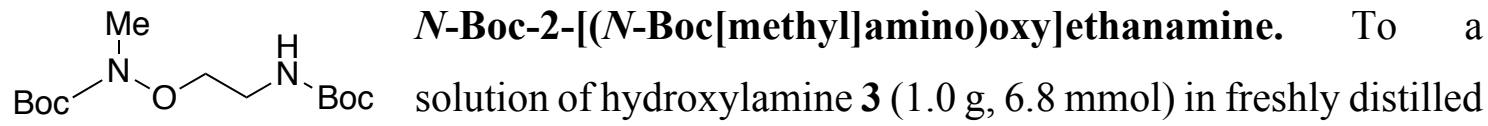
dry DMF ( $2 \mathrm{~mL}), \mathrm{NaH}(0.21 \mathrm{~g}, 8.8 \mathrm{mmol})$ was added and the reaction mixture was stirred at $\mathrm{rt}$ under argon. After $1 \mathrm{~h}$, a solution of bromide $2(1.97 \mathrm{~g}, 8.8 \mathrm{mmol})$ in freshly distilled dry DMF $(2 \mathrm{~mL})$ was added and the reaction mixture was stirred at $\mathrm{rt}$ for an additional $4 \mathrm{~h}$. The reaction mixture was then extracted with EtOAc $(2 \times 10 \mathrm{~mL})$ and the combined organic layers were washed with sat. aq. $\mathrm{NaHCO}_{3}$ and $\mathrm{NaCl}$ before being dried over $\mathrm{MgSO}_{4}$. Purification of the crude material by silica gel column chromatography (PE/EtOAc, 92/8, v/v) yielded the title compound as a colourless oil $(1.04 \mathrm{~g}, 53 \%) . \mathrm{R}_{f}=$ 0.52 (PE/EtOAc, 3/1, v/v); IR (film) 3361, 2977, 2930, 1708, 1507, $1154 \mathrm{~cm}^{-1}$; ${ }^{1} \mathrm{H}$ NMR $\left(500 \mathrm{MHz}, \mathrm{CDCl}_{3}\right) \delta 5.44(\mathrm{bs}, 1 \mathrm{H}, \mathrm{NH}), 3.85\left(\mathrm{t}, 2 \mathrm{H}, J_{\mathrm{l-2}}=4.8 \mathrm{~Hz}, \mathrm{CH}_{2} \mathrm{O}\right), 3.32(\mathrm{dt}, 2 \mathrm{H}$, $\left.J_{2, N H}=J_{l, 2}=4.8 \mathrm{~Hz}, \mathrm{H}-2\right), 3.08\left(\mathrm{~s}, 3 \mathrm{H}, \mathrm{N}-\mathrm{CH}_{3}\right), 1.50\left(\mathrm{~s}, 9 \mathrm{H}, \mathrm{CH}_{3} t \mathrm{Bu}\right), 1.43\left(\mathrm{~s}, 9 \mathrm{H}, \mathrm{CH}_{3}\right.$ $t \mathrm{Bu}) ;{ }^{13} \mathrm{C}$ NMR (125 MHz, $\left.\mathrm{CDCl}_{3}\right) \delta 157.4(\mathrm{C}=\mathrm{ONO}), 156.1(\mathrm{C}=\mathrm{ONH}), 82.1(\mathrm{Cq}), 79.4$ $(\mathrm{Cq}), 73.3\left(\mathrm{CH}_{2} \mathrm{O}\right), 39.0\left(\mathrm{CH}_{2} \mathrm{~N}\right), 36.6\left(\mathrm{NCH}_{3}\right), 28.5\left(\mathrm{CH}_{3} t \mathrm{Bu}\right), 28.4\left(\mathrm{CH}_{3}, t \mathrm{Bu}\right)$; HRMS(ESI) m/z calcd. for $\left[\mathrm{C}_{13} \mathrm{H}_{27} \mathrm{~N}_{2} \mathrm{O}_{5}\right]^{+}: 291.1914$, obsd.: 291.1914.<smiles>C[N+]([O-])([O-])OCCN</smiles>

2-[(Methylamino)oxy]ethanamine (4). A solution of TFA: $\mathrm{CH}_{2} \mathrm{Cl}_{2}$ (17 $\mathrm{mL}, \quad 1: 1 \mathrm{v} / \mathrm{v})$ was added to $N$-Boc-2-[(N-Boc[methyl]amino)oxy]ethanamine (3) (1.0 g, $3.46 \mathrm{mmol})$ and stirred at $\mathrm{rt}$ for $2 \mathrm{~h}$. The solvents were removed under reduced pressure to give amine $\mathbf{4}$ as the TFA salt, as a colourless oil (0.65 g, quant.). $\mathrm{R}_{f}=0.23\left(\mathrm{CH}_{2} \mathrm{Cl}_{2} / \mathrm{MeOH}, 4 / 1, \mathrm{v} / \mathrm{v}\right)$; IR (film) 2928, 1437, 1192, $1197 \mathrm{~cm}^{-1}$; ${ }^{1} \mathrm{H}$ NMR $\left(500 \mathrm{MHz}, \mathrm{D}_{2} \mathrm{O}\right) \delta 4.36\left(\mathrm{t}, 2 \mathrm{H}, J_{l, 2}=4.4 \mathrm{~Hz}, \mathrm{CH}_{2} \mathrm{O}\right), 3.33\left(\mathrm{t}, 2 \mathrm{H}, J_{2,1}\right.$ $\left.=4.9 \mathrm{~Hz}, \mathrm{CH}_{2} \mathrm{~N}\right), 3.01\left(\mathrm{~s}, 3 \mathrm{H}, \mathrm{CH}_{3}-\mathrm{NH}\right) ;{ }^{13} \mathrm{C} \mathrm{NMR}\left(125 \mathrm{MHz}, \mathrm{D}_{2} \mathrm{O}\right) \delta 72.0(\mathrm{C}-1), 39.9$ (C-2), $37.6\left(\mathrm{CH}_{3}-\mathrm{NH}\right)$; HRMS(ESI) $\mathrm{m} / \mathrm{z}$ calcd. for $\left[\mathrm{C}_{3} \mathrm{H}_{11} \mathrm{~N}_{2} \mathrm{O}\right]^{+}$: 91.0866, obsd.: 91.0869.

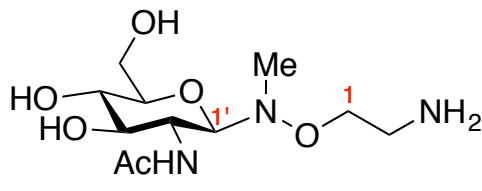

2-[(Methyl[ $N$-(2-acetamido-2-deoxy- $\beta$-Dglucopyranosyl)]-amino)oxy]ethanamine (24). $\mathrm{N}$ Acetylglucosamine (8.8 $\mathrm{mg}, \quad 0.04 \mathrm{mmol})$ and 2- 
[(methylamino)-oxy]ethanamine 4 (36 mg, $0.40 \mathrm{mmol})$ were reacted in an $\mathrm{AcOH} / \mathrm{NaOAc}$ buffer $(0.4 \mathrm{~mL}, 2 \mathrm{M}$, freshly prepared, $\mathrm{pH} 4.5)$ at $\mathrm{rt}$ for $24 \mathrm{hr}$. The crude mixture was then directly loaded onto a size exclusion column (Bio-Gel P-2, $1200 \times 18 \mathrm{~mm}$ ) and eluted with a $0.1 \mathrm{M}$ aq. $\mathrm{NH}_{4} \mathrm{HCO}_{2}$ solution. Lyophilisation of the product fractions afforded glycoside 24 as a white solid $(9.7 \mathrm{mg}, 83 \%) . \mathrm{R} f=0.19(t-\mathrm{BuOH} / \mathrm{EtOAc} / \mathrm{AcOH} / \mathrm{Pyr}$, $1 / 1 / 1 / 1, \mathrm{v} / \mathrm{v} / \mathrm{v} / \mathrm{v}) ; \alpha_{\mathrm{D}}{ }^{20.1}=-20(c=0.1, \mathrm{MeOH}) ; \mathrm{IR}($ film) 3269, 3039, 2931, 2883, 1651, 1561, 1408, $1379 \mathrm{~cm}^{-1}$; ${ }^{1} \mathrm{H}$ NMR (500 MHz, $\left.\mathrm{D}_{2} \mathrm{O}\right) \delta 4.19$ (d, $\left.1 \mathrm{H}, J_{l^{\prime}, 2^{\prime}}=9.8 \mathrm{~Hz}, \mathrm{H}-1^{\prime}\right)$, $3.99\left(\mathrm{t}, 1 \mathrm{H}, J_{1^{\prime}, 2^{\prime}}=J_{2^{\prime}, 3^{\prime}}=10.0 \mathrm{~Hz}, \mathrm{H}-2^{\prime}\right), 3.95-3.91\left(\mathrm{~m}, 2 \mathrm{H}, \mathrm{H}-1 \mathrm{a}, \mathrm{H}-6 \mathrm{a}^{\prime}\right), 3.80-3.70$ (m, 2H, H-1b, H-6b'), 3.55 - 3.45 (m, 1H, H-3'), 3.44 - 3.38 (m, 2H, H-4', H-5'), 3.24 $3.13\left(\mathrm{~m}, 2 \mathrm{H}, \mathrm{CH}_{2} \mathrm{~N}\right), 2.75\left(\mathrm{~s}, 3 \mathrm{H}, N-\mathrm{CH}_{3}\right), 2.02$ (s, 3H, $\left.\mathrm{CH}_{3} N \mathrm{NAc}\right) ;{ }^{13} \mathrm{C} \mathrm{NMR}(125 \mathrm{MHz}$, $\left.\mathrm{D}_{2} \mathrm{O}\right) \delta 170.8(\mathrm{C}=\mathrm{O}), 91.6\left(\mathrm{C}^{\prime} 1^{\prime}\right), 77.5\left(\mathrm{C}-5^{\prime}\right), 75.2\left(\mathrm{C}-3^{\prime}\right), 69.6\left(\mathrm{C}-4^{\prime}\right), 67.6\left(O-\mathrm{CH}_{2}-1\right)$, $60.6\left(\mathrm{C}-6^{\prime}\right), 52.0\left(\mathrm{C}-2^{\prime}\right), 38.7\left(\mathrm{NCH}_{3}\right), 38.2\left(N-\mathrm{CH}_{2}-2\right), 22.2\left(\mathrm{CH}_{3} N A c\right) ; \mathrm{HRMS}(\mathrm{ESI}) \mathrm{m} / z$ calcd. for $\left[\mathrm{C}_{11} \mathrm{H}_{24} \mathrm{~N}_{3} \mathrm{O}_{6}\right]^{+}: 294.1660$, obsd.:294.1665.

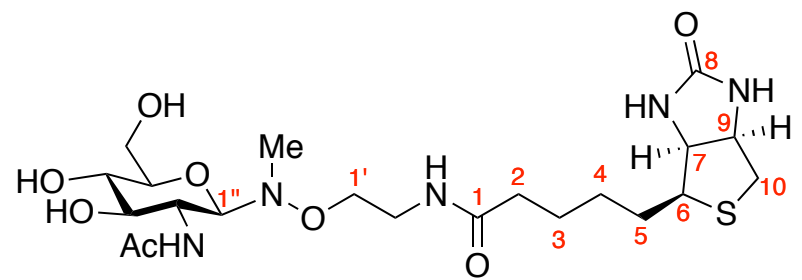

\section{$N$-(2-(Methyl[ $N$-(2-acetamido-2- deoxy- $\beta$-D-glucopyranosyl]-} aminooxy)ethyl)-D-biotinamide (26).

To a solution of amine $24(68.4 \mathrm{mg}$, $0.2 \mathrm{mmol})$ dissolved in DMF (2 mL), HBTU (150 mg, $0.35 \mathrm{mmol})$ and $\mathrm{Et}_{3} \mathrm{~N}(0.11 \mathrm{~mL}$, $0.8 \mathrm{mmol}$ ) were added. The resulting reaction mixture was stirred at $\mathrm{rt}$ for $2 \mathrm{~h}$. $\mathrm{H} 2 \mathrm{O}$ $(10 \mathrm{~mL})$ was then added to the reaction mixture and the white precipitate was filtered off and washed with water $(2 \times 5 \mathrm{~mL})$. The eluent was then purified using reverse phase chromatography (elute in $\mathrm{H}_{2} \mathrm{O} / \mathrm{MeOH}, 4: 1$ ) and lyophilised to afford glycoconjugate 26 as a colourless oil (45.5 mg, 44\%). $\mathrm{R}_{f}=0.59(t$-BuOH/EtOAc/AcOH/Pyr, 1/1/1/1, $\mathrm{v} / \mathrm{v} / \mathrm{v} / \mathrm{v}) ; \alpha_{\mathrm{D}}^{20.1}=+3.0(c=0.2, \mathrm{MeOH}) ;$ IR (film) 3340, 2931, 2852, 1651, $1560 \mathrm{~cm}^{-1}$; ${ }^{1} \mathrm{H}$ NMR $\left(500 \mathrm{MHz}, \mathrm{CDCl}_{3}\right) \delta 4.58\left(\mathrm{dd}, J_{7,6}=5.1, J_{9,7}=7.9 \mathrm{~Hz} 1 \mathrm{H}, \mathrm{H}-7\right), 4.40(\mathrm{~m}, 1 \mathrm{H}$, H-9), 4.20 (d, $\left.J_{l^{\prime \prime}, 2^{\prime \prime}}=10.5 \mathrm{~Hz}, 1 \mathrm{H}, \mathrm{H}-1^{\prime \prime}\right), 3.94$ (t, $\left.J_{2^{\prime \prime}, l^{\prime \prime}}=J_{2^{\prime \prime}, 3^{\prime \prime}}=10.5 \mathrm{~Hz}, 1 \mathrm{H}, \mathrm{H}-2^{\prime \prime}\right), 3.90$ $\left(\mathrm{m}, 1 \mathrm{H}, \mathrm{H}-6 \mathrm{a}^{\prime \prime}\right), 3.78\left(\mathrm{~m}, 1 \mathrm{H}, \mathrm{H}-1 \mathrm{a}^{\prime}\right), 3.74\left(\mathrm{dd}, J_{5^{\prime \prime}, 6 a^{\prime \prime}}=4.96, J_{6 b^{\prime \prime}, 6 a^{\prime \prime}}=12.3 \mathrm{~Hz}, 1 \mathrm{H}, \mathrm{H}-\right.$ $\left.6 \mathrm{a}^{\prime \prime}\right), 3.65\left(\mathrm{~m}, 1 \mathrm{H}, \mathrm{H}-1 \mathrm{~b}^{\prime}\right), 3.50\left(\mathrm{t}, J_{2^{\prime \prime}, 3^{\prime \prime}}=J_{4^{\prime \prime}, 3^{\prime \prime}}=9.7 \mathrm{~Hz}, 1 \mathrm{H}, \mathrm{H}-3^{\prime \prime}\right), 3.39$ (m, 3H, H-4", H-5", H-2a'), 3.30 (m, 2H, H-2b', H-6), 2.97 (dd, $J_{9,10 a}=4.9, J_{10 b, 10 a}=13.2 \mathrm{~Hz}, 1 \mathrm{H}, \mathrm{H}-$ $10 \mathrm{a}), 2.76\left(\mathrm{dd}, J_{9,10 b}=4.0, J_{10 a, 10 b}=13.2 \mathrm{~Hz}, 1 \mathrm{H}, \mathrm{H}-10 \mathrm{~b}\right), 2.71\left(\mathrm{~s}, 3 \mathrm{H}, N \mathrm{CH}_{3}\right), 2.27\left(\mathrm{t}, J_{3,2}\right.$ $=7.4 \mathrm{~Hz}, 2 \mathrm{H}, \mathrm{H}-2), 2.02\left(\mathrm{~s}, 3 \mathrm{H}, \mathrm{CH}_{3} \mathrm{Ac}\right), 1.75-1.53(\mathrm{~m}, 4 \mathrm{H}, \mathrm{H}-3, \mathrm{H}-5), 1.40$ (quin, $J_{3,4}=$ $\left.J_{5,4}=6.8 \mathrm{~Hz}, 2 \mathrm{H}, \mathrm{H}-4\right) ;{ }^{13} \mathrm{C}$ NMR $\left(125 \mathrm{MHz}, \mathrm{CDCl}_{3}\right) \delta 176.8(\mathrm{C}=\mathrm{O} \mathrm{C}-1), 174.1(\mathrm{C}=\mathrm{O}$ Ac), 165.2 (C=O C-8), 91.5 (C-1"), 77.3 (C-5"), 75.1 (C-3"), $70.1\left(\mathrm{C}-1^{\prime}\right), 69.5\left(\mathrm{C}-4^{\prime \prime}\right)$, 
62.0 (C-7), 60.7 (C-6"), 60.1 (C-9), 55.1 (C-6), 52.1 (C-2"), 39.6 (C-10), $38.4\left(\mathrm{NCH}_{3}\right)$, 38.1 (C-2'), 35.3 (C-2), 27.7 (C-4), 27.5 (C-5), 25.0 (C-3), 22.2 ( $\mathrm{CH}_{3}$ Ac); HRMS(ESI) $m / z$ calcd. for $\left[\mathrm{C}_{21} \mathrm{H}_{37} \mathrm{~N}_{5} \mathrm{O}_{8} \mathrm{SNa}\right]^{+}: 542.2255$, obsd.: 542.2265 .

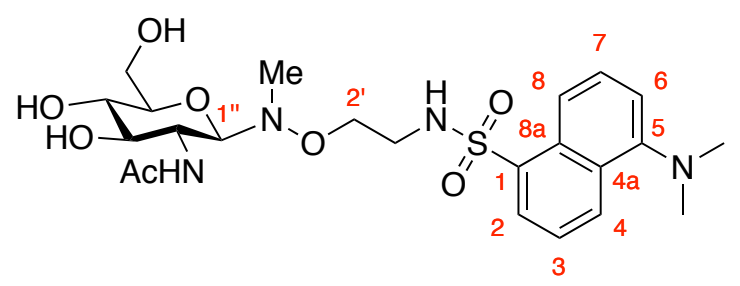

5-(Dimethylamino)- $N-(2-(($ methyl(2acetamido-2-deoxy- $\beta$-D-glucopyranosyl)amino)oxy)ethyl)naphthalene-1-sulfonamide (28). To a

solution of amine glycoside $24(8.3 \mathrm{mg}, 0.028 \mathrm{mmol})$ in $\mathrm{H}_{2} \mathrm{O}(2 \mathrm{~mL}), \mathrm{NaHCO}_{3}$ $(0.17 \mathrm{mmol}, 0.014 \mathrm{~g})$ was added. Dansyl chloride $(0.1 \mathrm{~g}, 0.042 \mathrm{mmol})$ was dissolved in freshly distilled THF $(1 \mathrm{~mL})$ and added dropwise to the reaction mixture. After $2 \mathrm{~h}$ the THF was concentrated in vacuo and the remaining aqueous reaction mixture was purified by reverse phase column chromatography $\left(\mathrm{C}_{18}, \mathrm{H}_{2} \mathrm{O} / \mathrm{MeOH}, 100 / 0 \rightarrow 60 / 40\right.$, v/v) followed by silica gel column chromatography $\left(\mathrm{CH}_{2} \mathrm{Cl}_{2} / \mathrm{MeOH}, 100 / 0 \rightarrow 90 / 10\right.$, v/v) to afford the fluorescent glycoside as an oil $(10 \mathrm{mg}, 70 \%) . \mathrm{R} f=0.61\left(\mathrm{CH}_{2} \mathrm{Cl}_{2} / \mathrm{MeOH}, 4 / 1, \mathrm{v} / \mathrm{v}\right) ; \alpha_{\mathrm{D}}{ }^{20.3}$ $=-10(c=0.1, \mathrm{MeOH})$; IR (film) 3035, 2931, 2986, 2040, $1719 \mathrm{~cm}^{-1}$; UV-VIS: (MeOH, $\left.5.70 \times 10^{-5} \mathrm{M}\right), \lambda_{\text {Abs } \max }=331 \mathrm{~nm}, \varepsilon=1684 \mathrm{M}^{-1} \mathrm{~cm}^{-1}(3.23) ;{ }^{1} \mathrm{H}$ NMR $\left(500 \mathrm{MHz}, \mathrm{D}_{2} \mathrm{O}\right)$ $\delta 8.52\left(\mathrm{~d}, 1 \mathrm{H}, J_{2,3}=8.6 \mathrm{~Hz}, \mathrm{H}-2\right), 8.33\left(\mathrm{~d}, 1 \mathrm{H}, J_{7,8}=8.8 \mathrm{~Hz}, \mathrm{H}-8\right), 8.28\left(\mathrm{~d}, 1 \mathrm{H}, J_{3,4}=7.4\right.$ $\mathrm{Hz}, \mathrm{H}-4), 7.74$ (t, $\left.1 \mathrm{H}, J_{3,4}=J_{2,3}=7.7 \mathrm{~Hz}, \mathrm{H}-3\right), 7.71$ (t, 1H, $J_{6,7}=J_{7,8}=7.7 \mathrm{~Hz}, \mathrm{H}-7$ ), 7.44 $\left(\mathrm{d}, 1 \mathrm{H}, J_{6,7}=7.7 \mathrm{~Hz}, \mathrm{H}-6\right), 4.07\left(\mathrm{~d}, 1 \mathrm{H}, J_{l^{\prime \prime}, 2^{\prime \prime}}=9.7 \mathrm{~Hz}, \mathrm{H}-1^{\prime}\right), 3.88\left(\mathrm{~d}, 1 \mathrm{H}, J_{6 a^{\prime \prime}, 6 b^{\prime \prime}}=\right.$ $\left.12.0 \mathrm{~Hz}, \mathrm{H}-6 \mathrm{~b}^{\prime \prime}\right), 3.81\left(\mathrm{t}, 1 \mathrm{H}, J_{l^{\prime \prime}, 2^{\prime \prime}}=J_{2^{\prime \prime}, 3^{\prime \prime}}=9.8 \mathrm{~Hz}, \mathrm{H}-2^{\prime \prime}\right), 3.71\left(\mathrm{dd}, 1 \mathrm{H}, J_{6 a^{\prime \prime}, 5^{\prime \prime}}=5.7 \mathrm{~Hz}\right.$, $\left.J_{6 a^{\prime \prime}, 6 b^{\prime \prime}}=12.3 \mathrm{~Hz}, \mathrm{H}-6 \mathrm{a}^{\prime \prime}\right), 3.51-3.40$ (m, 3H, NO-CH $\left.{ }_{2}-2^{\prime}, \mathrm{H}-3^{\prime \prime}\right), 3.36\left(\mathrm{t}, 1 \mathrm{H}, J_{4^{\prime \prime}, 5^{\prime \prime}}=J_{3^{\prime \prime}, 4^{\prime \prime}}\right.$ $\left.=9.3 \mathrm{~Hz}, \mathrm{H}-4^{\prime \prime}\right), 3.32-3.25$ (m, 1H, H-5"), $3.18-3.09$ (m, 2H, H-1'), 2.88 (s, 6H, N$\left.\left(\mathrm{CH}_{3}\right)_{2}\right), 2.30\left(\mathrm{~s}, 3 \mathrm{H}, \mathrm{CH}_{3} \mathrm{Ac}\right), 1.97\left(\mathrm{~s}, 3 \mathrm{H}, \mathrm{N}-\mathrm{CH}_{3}\right) ;{ }^{13} \mathrm{C} \mathrm{NMR}\left(125 \mathrm{MHz}, \mathrm{D}_{2} \mathrm{O}\right) \delta 173.9$ $(\mathrm{C}=\mathrm{O}), 150.7$ (C-4a), 134.0 (C-5), 130.1 (C-2), 129.5 (C-4), 128.9 (C-7), 128.8 (C-8a, C1), 124.0 (C-3), 119.1 (C-8), 116.0 (C-6), 91.4 (C-1"), 77.3 (C-5"'), 75.2 (C-3"), 69.8, 69.6 (C-4", C-1'), $60.8\left(\mathrm{C}-6^{\prime \prime}\right), 52.1\left(\mathrm{C}-2^{\prime \prime}\right), 44.9\left(N-\left(\mathrm{CH}_{3}\right)_{2}\right), 41.3\left(\mathrm{C}-2^{\prime}\right), 37.6\left(\mathrm{CH}_{3} N A \mathrm{c}\right)$, $22.1\left(N-\mathrm{CH}_{3}\right)$; HRMS(ESI) m/z calcd. for $\left[\mathrm{C}_{23} \mathrm{H}_{35} \mathrm{~N}_{4} \mathrm{O}_{8} \mathrm{~S}\right]^{+}:$527.2170, obsd.: 527.2178.

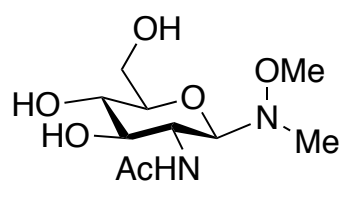

$N$-(2-Acetamido-2-deoxy- $\beta$-D-glucopyranosyl)- $N$-methyl- $O$ methylhydroxylamine (37). $N$-Acetylglucosamine $(1.0 \mathrm{~g}$, $4.5 \mathrm{mmol})$ and $\mathrm{N}, \mathrm{O}$-dimethylhydroxylamine hydrochloride $(1.3 \mathrm{~g}$, $13 \mathrm{mmol}$ ) were dissolved in $\mathrm{AcOH} / \mathrm{NaOAc}$ buffer (45 mL, $2 \mathrm{M}, \mathrm{pH} 4.5)$ at rt. After $24 \mathrm{~h}$, 
$\mathrm{NH}_{4} \mathrm{HCO}_{2}$ was added to the reaction mixture, which was then lyophilised. The crude material was purified by silica gel column chromatography $\left(15 \% \mathrm{MeOH}\right.$ in $\left.\mathrm{CH}_{2} \mathrm{Cl}_{2}, \mathrm{v} / \mathrm{v}\right)$, followed by ion exchange (Dowex- $\mathrm{OH}^{-}$) to afford the title compound as a white solid $(0.77 \mathrm{~g}, 65 \%) . \mathrm{R}_{f}=0.26\left(\mathrm{MeOH} / \mathrm{CH}_{2} \mathrm{Cl}_{2}, 1: 4, \mathrm{v} / \mathrm{v}\right) ; \alpha_{\mathrm{D}}{ }^{20.1}=-10(c=0.1, \mathrm{MeOH}) ; \mathrm{IR}$ (film) 3290, 2938, 2895, 1647, 1561, $1035 \mathrm{~cm}^{-1} ;{ }^{1} \mathrm{H}$ NMR (500 MHz, $\left.\mathrm{D}_{2} \mathrm{O}\right) \delta 4.19(\mathrm{~d}, 1 \mathrm{H}$, $\left.J_{1,2}=9.6 \mathrm{~Hz}, \mathrm{H}-1\right), 3.99\left(\mathrm{t}, 1 \mathrm{H}, J_{1,2}=J_{2,3}=9.8 \mathrm{~Hz}, \mathrm{H}-2\right), 3.93\left(\mathrm{dd}, 1 \mathrm{H}, J_{6 a, 6 b}=12.3 \mathrm{~Hz}\right.$, $\left.J_{5,6 a}=2.2 \mathrm{~Hz}, \mathrm{H}-6 \mathrm{a}\right), 3.75\left(\mathrm{dd}, 1 \mathrm{H}, J_{6 b, 6 a}=12.3 \mathrm{~Hz}, J_{5,6 b}=5.5 \mathrm{~Hz}, \mathrm{H}-6 \mathrm{~b}\right), 3.52(\mathrm{dd}, 1 \mathrm{H}$, $\left.J_{2,3}=J_{3,4}=10.0 \mathrm{~Hz}, \mathrm{H}-3\right), 3.46\left(\mathrm{~s}, 3 \mathrm{H}, \mathrm{OCH}_{3}\right), 3.45-3.40(\mathrm{~m}, 2 \mathrm{H}, \mathrm{H}-4, \mathrm{H}-5), 2.74(\mathrm{~s}$, $\left.3 \mathrm{H}, \mathrm{NCH}_{3}\right), 2.05$ (s, 3H, $\left.\mathrm{CH}_{3} \mathrm{Ac}\right) ;{ }^{13} \mathrm{C} \mathrm{NMR}\left(125 \mathrm{MHz}, \mathrm{D}_{2} \mathrm{O}\right) \delta 173.9(\mathrm{C}=\mathrm{O} \mathrm{Ac}), 91.2$ (C-1), 77.4 (C-5), 75.3 (C-3), 69.6 (C-4), 60.8 (C-6), $49.3\left(\mathrm{OCH}_{3}\right), 52.1$ (C-2), 38.1 $\left(\mathrm{NCH}_{3}\right), 22.2\left(\mathrm{CH}_{3} \mathrm{Ac}\right)$; HRMS(ESI) $m / z$ calcd. for $\left[\mathrm{C}_{10} \mathrm{H}_{21} \mathrm{~N}_{2} \mathrm{O}_{6}\right]^{+}:$265.1394, obsd.: 265.1400 .

SH 1,1,1-Tris(mercaptomethyl)ethane (38). Sulfur powder (1.66 g, $\mathrm{HS} 乙 \mathrm{SH} 72 \mathrm{mmol})$ and $\mathrm{Na}_{2} \mathrm{~S} .9 \mathrm{H}_{2} \mathrm{O}(4.7 \mathrm{~g}, 36 \mathrm{mmol})$ were dissolved in dry DMF $(50 \mathrm{~mL})$ under argon, forming a dark maroon solution. The mixture was then heated to $80{ }^{\circ} \mathrm{C}$ for $1 \mathrm{~h}$, after which it turned deep blue. The reaction mix was then cooled to $40{ }^{\circ} \mathrm{C}$ before adding $52(6.5 \mathrm{~g}, 12 \mathrm{mmol})$. After the addition was complete, the brown reaction mixture was heated to $80{ }^{\circ} \mathrm{C}$ for $16 \mathrm{~h}$. The reaction mixture was then concentrated in vacuo, followed by the addition of toluene $(50 \mathrm{~mL})$ to the resulting orange paste. Copper powder $(1.71 \mathrm{~g}, 27 \mathrm{mmol})$ was then added to the mixture before heating it to reflux for $2 \mathrm{~h}$. The black $\mathrm{CuS}$ formed during the reaction was then removed by filtration. EtOH $(50 \mathrm{~mL})$ and zinc powder $(2.7 \mathrm{~g}, 41 \mathrm{mmol})$ were then added to the filtrate before the slow addition of concentrated $\mathrm{HCl}(20 \mathrm{~mL})$. The resulting mixture was placed under an atmosphere of argon and stirred at $\mathrm{rt}$ for $16 \mathrm{~h}$. The zinc powder was removed by filtration and the filtrate was then extracted with toluene $(3 \times 20 \mathrm{~mL})$, and the combined organic layers washed with water $(20 \mathrm{~mL})$ and brine, dried $\left(\mathrm{MgSO}_{4}\right)$, filtered and concentrated. The crude material was purified using silica gel chromatography using petroleum ether to yield the title compound as a bright yellow oil with a pungent smell $(0.83 \mathrm{~g}, 42 \%)$. $\mathrm{R}_{f}$ $=0.79(\mathrm{PE} /$ EtOAc, $3: 1, \mathrm{v} / \mathrm{v}) ;$ IR (film) 2960, 2919, 2554, 1459, $1414 \mathrm{~cm}^{-1} ;{ }^{1} \mathrm{H}$ NMR (500 $\left.\mathrm{MHz}_{\mathrm{CDCl}}\right) \delta 2.61\left(\mathrm{~d}, J_{\mathrm{SH}, \mathrm{CH} 2}=8.8 \mathrm{~Hz}, 6 \mathrm{H}, \mathrm{CH}_{2}\right), 1.22\left(\mathrm{t}, J_{\mathrm{CH}, \mathrm{SH}}=8.8 \mathrm{~Hz}, 3 \mathrm{H}, \mathrm{SH}\right)$, $1.01\left(\mathrm{~s}, 3 \mathrm{H}, \mathrm{CH}_{3}\right) ;{ }^{13} \mathrm{C} \mathrm{NMR}\left(125 \mathrm{MHz}, \mathrm{CDCl}_{3}\right) \delta 39.2(\mathrm{Cq}), 31.8\left(\mathrm{CH}_{2}\right), 21.5\left(\mathrm{CH}_{3}\right)$; HRMS(ESI) $m / z$ calcd. For M-2H-H $\left[\mathrm{C}_{5} \mathrm{H}_{9} \mathrm{~S}_{3}\right]^{-}: 164.9866$, obsd.: 164.9879. 


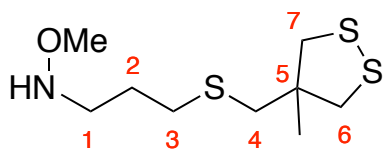

$O$-Methyl- $\mathrm{N}$-(2-(((4-methyl-1,2-dithiolan-4yl)methyl)thio)ethyl)hydroxylamine

(39).

Acrolein

(0.095 $\mathrm{mL}, 1.42 \mathrm{mmol})$ was added dropwise to neat $38(0.264 \mathrm{~g}$, $1.57 \mathrm{mmol}$ ). $2 \mathrm{~mL} \mathrm{CH}_{2} \mathrm{Cl}_{2}$ was then added to the mixture. The reaction mixture was heated to $40{ }^{\circ} \mathrm{C}$ o/n. After being cooled to rt, the reaction mixture was diluted with EtOH $(2.5 \mathrm{~mL})$ before methoxyamine hydrochloride was added $(0.14 \mathrm{~g}, 1.7 \mathrm{mmol})$. The mixture was then stirred at $\mathrm{rt} \mathrm{o} / \mathrm{n} . \mathrm{NaCNBH}_{3}(0.13 \mathrm{~g}, 2.13 \mathrm{mmol})$ was then added to the mixture along with the dropwise addition of ethanolic $\mathrm{HCl}(5 \mathrm{~mL}$, freshly prepared from $\mathrm{AcCl}$ and $\mathrm{EtOH}$ ) and left to stir at $\mathrm{rt}$ for $2 \mathrm{~h}$. The crude mixture was then concentrated in vacuo and suspended in sat. aq. $\mathrm{NaHCO}_{3}$ before being extracted with $\mathrm{CH}_{2} \mathrm{Cl}_{2}(3 \times 10 \mathrm{~mL})$. The combined organic layers were then washed with brine and dried over $\mathrm{MgSO}_{4}$. The crude material was purified using silica gel chromatography (PE/EtOAc, 94/6 v/v) to yield 39 as a yellow oil (99 mg, 27\%), as a 2:1 mixture of disulfide:dithiol (dithiol denoted with prime '). $\mathrm{R}_{f}=0.33\left(\mathrm{PE} /\right.$ EtOAc, 3:1, v/v); IR (film) 2934, 1458, 1373, $1123 \mathrm{~cm}^{-1} ;{ }^{1} \mathrm{H}$ NMR $\left(500 \mathrm{MHz}, \mathrm{CDCl}_{3}\right) \delta 3.52\left(\mathrm{~s}, 3 \mathrm{H}, \mathrm{OCH}_{3}\right), 3.12\left(\mathrm{~d}, J_{C H 2 b, C H 2 a}=11.8 \mathrm{~Hz}, 2 \mathrm{H}, \mathrm{H}-6 \mathrm{a}, \mathrm{H}-7 \mathrm{a}\right)$, $3.00\left(\mathrm{t}, J_{2,1}=6.9 \mathrm{~Hz}, 2 \mathrm{H}, \mathrm{H}-1\right), 2.89$ (d, $\left.J_{\mathrm{CH} 2 a, C H 2 b}=11.8 \mathrm{~Hz}, 2 \mathrm{H}, \mathrm{H}-6 \mathrm{~b}, \mathrm{H}-7 \mathrm{~b}\right), 2.76$ (s, 2H, H-4), 2.67-2.58 (m, 2 H, H-3 and H-4', H-6', H-7' from dithiol), 1.82 (m, 2H, H-2), $1.30\left(\mathrm{~s}, \mathrm{CH}_{3}\right), 1.03\left(\mathrm{~s}, \mathrm{CH}_{3}{ }^{\prime}\right.$ from dithiol); ${ }^{13} \mathrm{C} \mathrm{NMR}\left(125 \mathrm{MHz}, \mathrm{CDCl}_{3}\right) \delta 62.0\left(\mathrm{OCH}_{3}\right)$, 52.0 (C-5), 50.6 (C-1), 49.2 (C-6,7), 42.4 (C-4), 40.2 (C-4'), 39.5 (C-5'), 32.5 (C-6', C7'), 32.0 (C-3), 31.4 (C-3'), 27.5 (C-2), $24.5\left(\mathrm{CH}_{3}\right), 22.2\left(\mathrm{CH}_{3}{ }^{\prime}\right)$; HRMS(ESI) m/z calcd. for $\left[\mathrm{C}_{9} \mathrm{H}_{20} \mathrm{NOS}_{3}\right]^{+}: 254.0702$, obsd.: 254.0711.

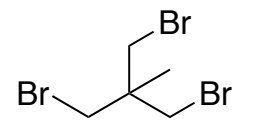

\section{1,1,1-Tris(bromomethyl)ethane}

(41).

$1,1,1-$ pyridine $(25 \mathrm{~mL})$ in a dry rond-bottom flask fitted with a reflux condenser, and placed under argon. $\mathrm{PBr}_{3}(7.8 \mathrm{~mL}, 83 \mathrm{mmol})$ was then added dropwise at a very slow rate $(1 \mathrm{~mL} / \mathrm{min})$ to the reaction mixture, producing a yellow suspension. After heating the mixture at reflux for $24 \mathrm{~h}$, the mixture was cooled to $\mathrm{rt}$ before ice was slowly added to quench the reaction. Water was then slowly added $(10 \mathrm{~mL})$ before extracting the reaction mixture with $\mathrm{CH}_{2} \mathrm{Cl}_{2}(3 \times 20 \mathrm{~mL})$. The combined organic layers were then washed with water $(10 \mathrm{~mL})$, sat. aq. $\mathrm{NaHCO}_{3}$ and brine, then dried $\left(\mathrm{MgSO}_{4}\right)$, filtered and concentrated. Purification of the crude material by silica gel chromatography using petroleum ether produced the title compound as a colourless liquid (357 mg, 27\%). $\mathrm{R}_{f}=0.92(\mathrm{PE} / \mathrm{EtOAc}$, 3:1, v/v); IR (film) 2971, 1464, 1277, $1029 \mathrm{~cm}^{-1} ;{ }^{1} \mathrm{H}$ NMR (500 MHz, $\left.\mathrm{CDCl}_{3}\right) \delta 3.50$ (s, 
6H, $\left.\mathrm{CH}_{2}\right), 1.28\left(\mathrm{~s}, 3 \mathrm{H}, \mathrm{CH}_{3}\right) ;{ }^{13} \mathrm{C} \mathrm{NMR}\left(125 \mathrm{MHz}, \mathrm{CDCl}_{3}\right) \delta 39.6(\mathrm{Cq}), 39.0\left(\mathrm{CH}_{2}\right), 21.6$ $\left(\mathrm{CH}_{3}\right)$.<smiles>SC(I)CCCC(S)[Hg]</smiles>

1,4-Butanedithiol (43). 1,4-Dibromobutane (1 g, $4.63 \mathrm{mmol})$ and thiourea $(0.78 \mathrm{~g}, 10.2 \mathrm{mmol})$ were dissolved in EtOH $(5 \mathrm{~mL})$. The reaction mixture was then placed under argon and heated to reflux for $3 \mathrm{~h}$. The mixture was then cooled for the addition of $\mathrm{NaOH}(2.5 \mathrm{M}, 6 \mathrm{~mL})$ and then heated to reflux once more for $2 \mathrm{~h}$. Once the reaction was complete, the mixture was cooled to $\mathrm{rt}$ and quenched with $\mathrm{HCl}(1 \mathrm{M}, 12 \mathrm{~mL})$. The quenched mixture was then extracted with petroleum ether $(3 \times 20 \mathrm{~mL})$. The combined organic layers were washed with brine and dried over $\mathrm{MgSO}_{4}$ and concentrated in vacuo to produce 43 as a yellow oil with a strong odour (415 $\mathrm{mg}$, $73 \%$ ). The crude material required no further purification. $\mathrm{R}_{f}=0.89(\mathrm{PE} / \mathrm{EtOAc}, 3: 1, \mathrm{v} / \mathrm{v})$; IR (film) 2926, 2851, 1738, $1448 \mathrm{~cm}^{-1}$; ${ }^{1} \mathrm{H}$ NMR (500 MHz, $\left.\mathrm{CDCl}_{3}\right) \delta 2.54$ (m, 4H, H-1, $\mathrm{H}-4), 1.71(\mathrm{~m}, 4 \mathrm{H}, \mathrm{H}-2, \mathrm{H}-3), 1.35\left(\mathrm{t}, J_{1+4, S H}=7.9 \mathrm{~Hz}, \mathrm{SH}\right) ;{ }^{13} \mathrm{C} \mathrm{NMR}\left(125 \mathrm{MHz}, \mathrm{CDCl}_{3}\right)$ $\delta 32.6$ (C-1, C-4), 24.2 (C-2, C-3); HRMS(ESI) $m / z$ calcd. For M-H ${ }^{-}\left[\mathrm{C}_{4} \mathrm{H}_{9} \mathrm{~S}_{2}\right]^{-}: 121.0146$, obsd.: 121.0151 .

$\mathrm{Br}$ 1,2,3-Tribromopropane (45). Allylbromide (2 g, $16.5 \mathrm{mmol})$ was $\mathrm{Br} \underbrace{\mathrm{Br}}_{3}$ dissolved in $\mathrm{CCl}_{4}(10 \mathrm{~mL})$. The mixture was cooled on ice before the dropwise addition of $\mathrm{Br}_{2}(2.2 \mathrm{~g}, 13.8 \mathrm{mmol})$. After the addition was complete, the mixture was stirred at $\mathrm{rt}$ for $1 \mathrm{~h}$. Concentration of the reaction mixture in vacuo yielded tribromopropane 45 as a lightly brown-tinged oil which was used without further purification (3.8 g, quant.). $\mathrm{R}_{f}=0.83$ (PE/EtOAc, 3:1, v/v); IR (film) 2924, 1737, 1448, $1217 \mathrm{~cm}^{-1}$; ${ }^{1} \mathrm{H}$ NMR $\left(500 \mathrm{MHz}, \mathrm{CDCl}_{3}\right) \delta 4.37(\mathrm{~m}, 1 \mathrm{H}, \mathrm{H}-2), 3.94\left(\mathrm{dd}, J_{1 b+3 b, 1 a+3 a}=\right.$ $\left.4.3 \mathrm{~Hz}, J_{2,1 a+3 a}=10.9 \mathrm{~Hz}, 2 \mathrm{H}, \mathrm{H}-1 \mathrm{a}, \mathrm{H}-3 \mathrm{a}\right), 3.84\left(\mathrm{dd}, J_{1 a+3 a, 1 b+3 b}=4.3 \mathrm{~Hz}, J_{2,1 b+3 b}=\right.$ $10.9 \mathrm{~Hz}, 2 \mathrm{H}, \mathrm{H}-1 \mathrm{~b}, \mathrm{H}-3 \mathrm{~b}) ;{ }^{13} \mathrm{C}$ NMR (125 MHz, $\left.\mathrm{CDCl}_{3}\right) \delta 48.3$ (C-2), 35.0 (C-1, C-3).

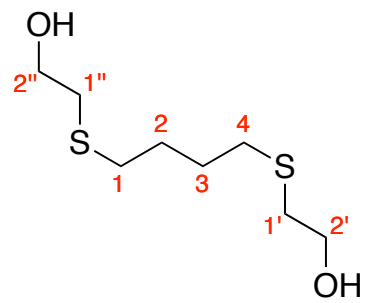

1,4-Bis(2-hydroxyethylthio)butane (49). Sodium metal (21 mg, $0.93 \mathrm{mmol})$ was dissolved in EtOH (1 mL) under an atmosphere or argon. Once the sodium was completely dissolved, mercaptoethanol $(72.7 \mathrm{mg}, 0.93 \mathrm{mmol})$ was added, followed by the dropwise addition of 1,4-dibromobutane (100 $\mathrm{mg}$, $0.46 \mathrm{mmol}$ ). The reaction was left to stir for $1 \mathrm{~h}$ before the mixture was concentrated in vacuo. The residue was then extracted with $\mathrm{CH}_{2} \mathrm{Cl}_{2}(3 \times 20 \mathrm{~mL})$. The combined organic 
layers were washed with water $(10 \mathrm{~mL})$ and brine before being dried over $\mathrm{MgSO}_{4}$. Removal of the solvent afforded the title compound as a light yellow oil, which did not require further purification $(78 \mathrm{mg}, 80 \%) . \mathrm{R}_{f}=0.31(\mathrm{PE} / \mathrm{EtOAc}, 1: 1, \mathrm{v} / \mathrm{v})$; IR (film) 3364, 2918, 2851, 1421, $1044 \mathrm{~cm}^{-1} ;{ }^{1} \mathrm{H}$ NMR (500 MHz, $\left.\mathrm{CDCl}_{3}\right) \delta 3.66$ (m, 4H, H-2', H-2"), $2.81(\mathrm{bs}, 2 \mathrm{H}, \mathrm{OH}), 2.66\left(\mathrm{t}, J_{2^{\prime}, l^{\prime}}=J_{2^{\prime \prime}, 1^{\prime \prime}}=6.1 \mathrm{~Hz}, 4 \mathrm{H}, \mathrm{H}-1^{\prime}, \mathrm{H}-1^{\prime \prime}\right) 2.49$ (m, 4H, H-1, H-4), 1.64 (m, 4H, H-2, H-3); ${ }^{13} \mathrm{C}$ NMR (125 MHz, $\left.\mathrm{CDCl}_{3}\right) \delta 60.2\left(\mathrm{C}-2^{\prime}, \mathrm{C}-2^{\prime \prime}\right), 35.2\left(\mathrm{C}-1^{\prime}, \mathrm{C}-\right.$ $\left.1^{\prime \prime}\right), 31.1$ (C-1, C-4), 28.5 (C-2, C-3); HRMS(ESI) $m / z$ calcd. for $\left[\mathrm{C}_{8} \mathrm{H}_{18} \mathrm{O}_{2} \mathrm{~S}_{2} \mathrm{~K}\right]^{+}$: 249.0380, obsd.: 249.0390 .

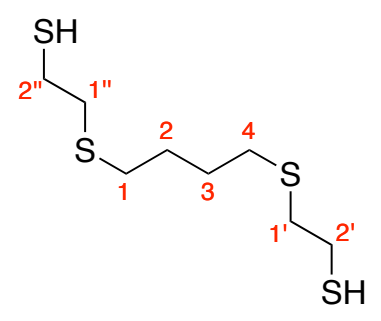

1,4-Bis(2-mercaptoethylthio)butane (50). Concentrated $\mathrm{HCl}$ $(0.4 \mathrm{~mL})$ was added to a mixture of thiourea $(59 \mathrm{mg}, 0.77 \mathrm{mmol})$ and diol 49 (75 $\mathrm{mg}, 0.35 \mathrm{mmol}$ ). The mixture was then heated to reflux for $3 \mathrm{~h}$. The mixture was then cooled before the addition of $\mathrm{NaOH}\left(94 \mathrm{mg}, 2.36 \mathrm{mmol}\right.$ dissolved in $1 \mathrm{~mL} \mathrm{H}_{2} \mathrm{O}$ ). After the addition was complete, the mixture was heated to reflux once more for $3 \mathrm{~h}$. After the reaction was complete, the mixture was cooled to $\mathrm{rt}$ and quenched with $1 \mathrm{M} \mathrm{HCl}(20 \mathrm{~mL})$ followed by an extraction using petroleum ether $(3 \times 20 \mathrm{~mL})$. The combined organic layers were washed with water, $(10 \mathrm{~mL})$ and brine before being dried over $\mathrm{MgSO}_{4}$. The solvent was removed in vacuo to provide the title compound as a yellow oil which did not require further purification (42 mg, 50\%). $\mathrm{R}_{f}=0.75(\mathrm{PE} / \mathrm{EtOAc}, 3: 1, \mathrm{v} / \mathrm{v})$; IR (film) 2918, 2847, 1420, $1187 \mathrm{~cm}^{-1} ;{ }^{1} \mathrm{H}$ NMR (500 MHz, $\left.\mathrm{CDCl}_{3}\right) \delta 2.87$ (m, 4H, H-2', H-2"), 2.75 (m, 4H, H-1', H-1"), 2.56 (m, 4H, H-1, H-4), 1.71 (m, 4H, H-2, H-3); HRMS(ESI) $m / z$ calcd. for $\left[\mathrm{C}_{8} \mathrm{H}_{22} \mathrm{~S}_{4} \mathrm{~N}\right]^{+}: 260.0630$, obsd.: 260.0635 .

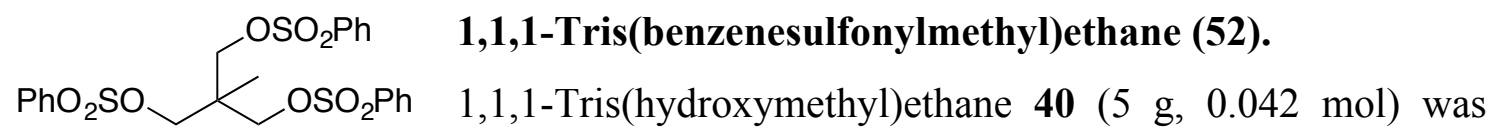
dissolved in dry pyridine $(20 \mathrm{~mL})$ under an atmosphere of argon. Once dissolved, the mixture was placed in an ice water bath before the dropwise addition of benzenesulfonyl chloride $(24.3 \mathrm{~g}, 0.137 \mathrm{~mol})$. The resulting white slurry was then stirred at $40{ }^{\circ} \mathrm{C}$ for $2 \mathrm{~h}$. The slurry was then poured slowly into a vigorously stirring solution of $40 \mathrm{~mL}$ concentrated $\mathrm{HCl}, 50 \mathrm{~mL}$ water and $100 \mathrm{~mL} \mathrm{MeOH}$. The resulting suspension containing Tris-benzenesulphonate as a white solid was then cooled on ice, filtered and washed with water and cold $\mathrm{MeOH}$ to provide the title compound as a white, crystalline solid which was used without further purification (19.5 g, 96\%). $\mathrm{R}_{f}=0.71(\mathrm{PE} / \mathrm{EtOAc}, 1: 1, \mathrm{v} / \mathrm{v}) ; \mathrm{Mp}$ 
92.7-96. ${ }^{\circ} \mathrm{C}$; IR (film) 3066, 1448, 1361, $1174 \mathrm{~cm}^{-1} ;{ }^{1} \mathrm{H}$ NMR $\left(500 \mathrm{MHz}, \mathrm{CDCl}_{3}\right) \delta 7.83$ $\left(\mathrm{d}, J_{m-P h, o-P h}=7.7 \mathrm{~Hz}, 6 \mathrm{H}, o-\mathrm{Ph}\right), 7.69(\mathrm{~m}, 3 \mathrm{H}, p-\mathrm{Ph}), 7.57(\mathrm{~m}, 6 \mathrm{H}, m-\mathrm{Ph}), 3.81(\mathrm{~s}, 6 \mathrm{H}$, $\left.\mathrm{CH}_{2}\right), 0.92\left(\mathrm{~s}, 3 \mathrm{H}, \mathrm{CH}_{3}\right) ;{ }^{13} \mathrm{C}$ NMR (125 MHz, D $\left.2 \mathrm{O}\right) \delta 134.3(p-\mathrm{Ph}), 129.6(m-\mathrm{Ph}), 128.0$ (o-Ph), $70.0\left(\mathrm{CH}_{2}\right), 16.2\left(\mathrm{CH}_{3}\right)$; HRMS(ESI) $\mathrm{m} / z$ calcd. for $\left[\mathrm{C}_{23} \mathrm{H}_{25} \mathrm{O}_{9} \mathrm{~S}_{3}\right]^{+}: 541.0655$, obsd.: 541.0678 .

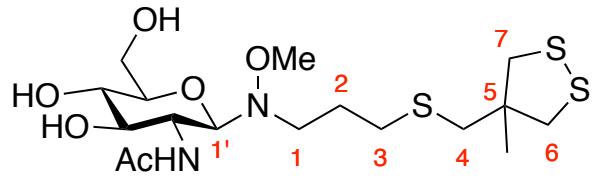

\section{$N$-(2-acetamido-2-deoxy- $\beta$-D-glucopyranosyl)- $O$-methyl- $N$-(2-(((4-methyl-1,2-dithiolan-4- yl)methyl)thio)hydroxylamine (56). $\quad \mathrm{N}$ -}

Acetylglucosamine (0.041 g, $0.184 \mathrm{mmol})$ and linker 39 (23.5 mg, $0.092 \mathrm{mmol})$ were reacted in $0.196 \mathrm{~mL}$ of a mixture of $\mathrm{AcOH} / \mathrm{NaOAc}$ buffer $(2 \mathrm{M}$, freshly prepared) and DMSO in a $1: 1$ solvent ratio $(\mathrm{v} / \mathrm{v})$ at $40{ }^{\circ} \mathrm{C}$ for $72 \mathrm{~h}$. The crude reaction mixture was directly loaded onto a size exclusion column (Bio-Gel P-2, $1200 \times 18 \mathrm{~mm}$ ) and eluted with a $0.1 \mathrm{M}$ aq. $\mathrm{NH}_{4} \mathrm{HCO}_{2}$ solution. Lyophilisation of the product fractions afforded glycoside 56 as an off-white solid (20.6 mg, 49\%). $\mathrm{R}_{f}=0.57\left(\mathrm{MeOH} / \mathrm{CH}_{2} \mathrm{Cl}_{2}, 1: 4, \mathrm{v} / \mathrm{v}\right)$; $\alpha_{\mathrm{D}}^{24.0}=-10(c=0.1, \mathrm{MeOH})$; IR (film) 3363, 2970, 1738, $1367 \mathrm{~cm}^{-1} ;{ }^{1} \mathrm{H}$ NMR (500 MHz, $\left.\mathrm{D}_{2} \mathrm{O}\right) \delta 4.31\left(\mathrm{~d}, J_{2^{\prime}, I^{\prime}}=9.6 \mathrm{~Hz}, 1 \mathrm{H}, \mathrm{H}-1^{\prime}\right), 3.87$ (m, 2H, H-2', H-6a'), $3.73\left(\mathrm{dd}, J_{5^{\prime}, 6 b^{\prime}}=4.6\right.$

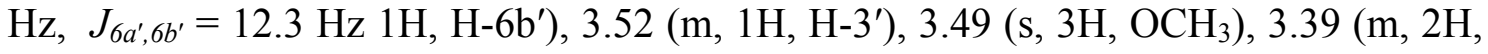
H-4', H-5'), 3.17 (d, $\left.J_{6 b+7 b, 6 a+7 a}=11.2 \mathrm{~Hz}, 2 \mathrm{H}, \mathrm{H}-6 \mathrm{a}, \mathrm{H}-7 \mathrm{a}\right), 3.03$ (m, 2H, H-1), 2.98 (d, $\left.J_{6 a+7 a, 6 b+7 b}=11.2 \mathrm{~Hz}, 2 \mathrm{H}, \mathrm{H}-6 \mathrm{~b}, \mathrm{H}-7 \mathrm{~b}\right), 2.84$ (s, 2H, H-4), 2.67 (m, 2H, H-2), 2.03 (s, 3H, $\left.\mathrm{CH}_{3} \mathrm{Ac}\right), 1.82(\mathrm{~m}, 2 \mathrm{H}, \mathrm{H}-3), 1.28\left(\mathrm{~s}, 3 \mathrm{H}, \mathrm{CH}_{3}\right) ;{ }^{13} \mathrm{C} \mathrm{NMR}\left(125 \mathrm{MHz}, \mathrm{D}_{2} \mathrm{O}\right) \delta 176.3(\mathrm{C}=\mathrm{O}$ Ac), $92.5\left(\mathrm{C}-1^{\prime}\right), 79.9\left(\mathrm{C}-5^{\prime}\right), 78.0\left(\mathrm{C}-3^{\prime}\right), 72.0\left(\mathrm{C}-4^{\prime}\right), 63.6\left(\mathrm{OCH}_{3}\right), 63.3\left(\mathrm{C}-6^{\prime}\right), 54.8(\mathrm{C}-$ 2'), 53.9 (C-5), 52.7 (C-1), 50.8 (C-6, C-7), 43.4 (C-4), 33.5 (C-2), 29.0 (C-3), $25.6\left(\mathrm{CH}_{3}\right)$, $24.7\left(\mathrm{CH}_{3} \mathrm{Ac}\right)$; HRMS(ESI) $m / z$ calcd. for $\left[\mathrm{C}_{17} \mathrm{H}_{32} \mathrm{~N}_{2} \mathrm{O}_{6} \mathrm{~S}_{3} \mathrm{~K}\right]^{+}: 479.1315$, obsd.:479.1337.

\subsection{Hydrolytic Stability Experiment}

A series of citric acid- $\mathrm{Na}_{2} \mathrm{HPO}_{4}$ buffers at various $\mathrm{pH}(\mathrm{pH} \mathrm{3,4,5,6)}$ and buffer concentrations $(25,50,100,200 \mathrm{mM})$, containing $0.1 \% \mathrm{NaN}_{3}$, were prepared. To the dansylated glycosides $\mathbf{2 8}$ and $\mathbf{2 9}$ (in separate flasks), a stock solution of internal standard 30 ( 0.25 equiv. compared to $\mathbf{2 8}$ or 29 ) in $\mathrm{H}_{2} \mathrm{O}$ was added and the resulting solutions were divided into separate reaction vessels and lyophilized to obtain $2 \mu \mathrm{mol}$ of dansylglycoside 28 or 29 per reaction vessel. To each reaction vessel, the previously prepared buffers (50-800 $\mu \mathrm{L}$, depending on experiment) were added. The hydrolysis reactions were 
monitored over time using HPLC-UV analysis (column: BEH300 $\mathrm{C}_{18} 5 \mu \mathrm{m}$, $4.0 \times 150 \mathrm{~mm}, 35 \% \mathrm{MeCN}$ in $\mathrm{H}_{2} \mathrm{O}$ ). The integrated chromatograms of glycosides 28 or 29 were measured against the integral of the internal standard. A line was fitted to the data according to the least-squares method, and the half-lives were calculated based on the fitted line. In control experiments, an excess of internal standard and $\mathrm{NaN}_{3}$ were added. In addition, reactions were performed in PBS at various glycan concentrations $(12.5,25,100,200 \mu \mathrm{M})$.

\section{4 $\mathrm{p} K_{\mathrm{a}}$ Determination}

${ }^{1} \mathrm{H}$ NMR spectra were recorded for $N$-methyl- $N$-(2-acetamido-2-deoxy- $\beta$-Dglucopyranosyl)-O-methyl-hydroxylamine $(37)$ in $\mathrm{D}_{2} \mathrm{O}$ at various $\mathrm{pD}$ values. A stock solution of glycosyloxyamine $37\left(10 \mathrm{mg} / \mathrm{mL}\right.$ in $\left.\mathrm{D}_{2} \mathrm{O}\right)$, and of $3 \mathrm{M} \mathrm{DCl}, 6 \mathrm{M} \mathrm{DCl}$ and $9 \mathrm{M}$ $\mathrm{DCl}$ in $\mathrm{D}_{2} \mathrm{O}$ were prepared. To seven individual samples of $0.5 \mathrm{~mL}$ of the glycosyloxyamine 37 stock solution $(10 \mathrm{mg} / \mathrm{mL})$ were added $3 \mathrm{M} \mathrm{DCl}(50 \mu \mathrm{L}), 6 \mathrm{M} \mathrm{DCl}$ $(50 \mu \mathrm{L}), 9 \mathrm{M} \mathrm{DCl}(50 \mu \mathrm{L})$, conc. DCl $(50 \mu \mathrm{L})$, conc. DCl $(100 \mu \mathrm{L})$, conc. DCl $(150 \mu \mathrm{L})$ or conc. DCl $(200 \mu \mathrm{L})$. One sample contained glycosyloxyamine 37 stock solution only, while the final sample contained glycosyloxyamine $37(5 \mathrm{mg})$ dissolved in conc. $\mathrm{DCl}(0.5$ $\mathrm{mL}$ ), with 4,4-dimethyl-4-silapentane-1-sulfonic acid (DSS, $1 \mathrm{mg}$ ) as an internal standard. ${ }^{1} \mathrm{H}$ NMR spectra were recorded for each sample within 2 minutes of DCl addition. The $\mathrm{pD}$ of each sample was determined by titration of chloride using Mohr's method. ${ }^{102}$ Briefly, after NMR spectra were recorded, each sample was titrated against a $0.1 \mathrm{M}$ solution of $\mathrm{AgNO}_{3}$ using $\mathrm{K}_{2} \mathrm{CrO}_{4}$ as the indicator in order to determine the amount of $\mathrm{Cl}^{-}$ions in solution, from which the $\mathrm{pD}$ was then calculated. The change in chemical shift of the NMe, OMe and anomeric proton of glycosyloxyamine 37 relative to DSS were each plotted against $\mathrm{pD}$ separately. A dose response curve was fitted according to equation 5 (derived from equations 6 to 10, Chapter 3) using the least-squares method, which provided the $\mathrm{p} K_{\mathrm{a}}$ value of the protonated glycoside. The final $\mathrm{p} K_{\mathrm{a}}$ value was taken as the average of the $\mathrm{p} K_{\mathrm{a}}$ values determined for the individual NMR signals. 


\section{Appendix 1}

\section{References}

1. Morelli, L.; Poletti, L.; Lay, L., "Carbohydrates and Immunology: Synthetic Oligosaccharide Antigens for Vaccine Formulation," Eur. J. Org. Chem. 2011, 2011, 5723-5777.

2. Hart, G. W.; Copeland, R. J., "Glycomics Hits the Big Time," Cell 2010, 143, 672-676.

3. Apweiler, R.; Hermjakob, H.; Sharon, N., "On the Frequency of Protein Glycosylation, as Deduced From Analysis of the SWISS-PROT Database," Biochim. Biophys. Acta 1999, 1473, 4-8.

4. $\quad$ Ernst, B.; Magnani, J. L., "From Carbohydrate Leads to Glycomimetic Drugs," Nat. Rev. Drug. Discov. 2009, 8, 661-677.

5. Freeze, H. H., "Understanding Human Glycosylation Disorders: Biochemistry Leads the Charge," J. Biol. Chem. 2013, 288, 6936-6945.

6. Daniels, G., Human Blood Groups. 2nd ed.; Blackwell Science Ltd.: Oxford, England, 2002.

7. Alberts, B.; Johnson, A.; Lewis, J.; Raff, M.; Roberts, K.; Walter, P., Molecular Biology of the Cell. 5th ed.; Garland Science: New York, U S A, 2002.

8. Pankov, R.; Yamada, K. M., "Fibronectin at a Glance," J. Cell. Sci. 2002, 115, 3861-3863.

9. Braulke, T.; Bonifacino, J. S., "Sorting of Lysosomal Proteins," Biochim. Biophys. Acta 2009, 1793, 605-614.

10. Tiede, S.; Storch, S.; Lubke, T.; Henrissat, B.; Bargal, R.; Raas-Rothschild, A.; Braulke, T., "Mucolipidosis II is Caused by Mutations in GNPTA Encoding the $\alpha / \beta$ GlcNAc-1-phosphotransferase," Nat. Med. 2005, 11, 1109-1112.

11. Kumar, H.; Kawai, T.; Akira, S., "Pathogen Recognition by the Innate Immune System," Int .Rev. Immunol. 2011, 30, 16-34.

12. Takeuchi, O.; Akira, S., "Pattern Recognition Receptors and Inflammation," Cell 2010, 140, 805-820.

13. Berti, F.; Adamo, R., "Recent Mechanistic Insights on Glycoconjugate Vaccines and Future Perspectives," ACS Chem. Biol. 2013, 8, 1653-1663.

14. Boltje, T. J.; Buskas, T.; Boons, G. J., "Opportunities and Challenges in Synthetic Oligosaccharide and Glycoconjugate Research," Nat. Chem. 2009, 1, 611-622.

15. Peri, F.; Cipolla, L.; La Ferla, B.; Nicotra, F., "Glycoconjugate and Oligosaccharide Mimetics by Chemoselective Ligation," C. R. Chim. 2003, 6, 635-644.

16. Specker, D.; Wittmann, V., "Synthesis and Application of Glycopeptide and Glycoprotein Mimetics," Top. Curr. Chem. 2007, 267, 65-107.

17. Liang, P.-H.; Wu, C.-Y.; Greenberg, W. A.; Wong, C.-H., "Glycan Arrays: Biological and Medical Applications," Curr. Opin. Chem. Biol. 2008, 12, 86-92.

18. Oyelaran, O.; Gildersleeve, J. C., "Glycan Arrays: Recent Advances and Future Challenges," Curr. Opin. Chem. Biol. 2009, 13, 406-413.

19. Hudak, J. E.; Bertozzi, C. R., "Glycotherapy: New Advances Inspire a Reemergence of Glycans in Medicine," Chem. Biol. 2014, 21, 16-37. 
20. Chen, W. C.; Kawasaki, N.; Nycholat, C. M.; Han, S.; Pilotte, J.; Crocker, P. R.; Paulson, J. C., "Antigen Delivery to Macrophages Using Liposomal Nanoparticles Targeting Sialoadhesin/CD169," PLOS ONE 2012, 7, e39039.

21. Stocker, B. L.; Timmer, M. S., "Chemical Tools for Studying the Biological Function of Glycolipids," ChemBioChem 2013, 14, 1164-1184.

22. Schramm, V. L., "Transition States, Analogues, and Drug Development," ACS Chem. Biol. 2013, 8, 71-81.

23. García-Vallejo, J. J.; Ambrosini, M.; Overbeek, A.; van Riel, W. E.; Bloem, K.; Unger, W. W.; Chiodo, F.; Bolscher, J. G.; Nazmi, K.; Kalay, H.; van Kooyk, Y., "Multivalent Glycopeptide Dendrimers for the Targeted Delivery of Antigens to Dendritic Cells," Mol. Immunol. 2013, 53, 387-397.

24. Arosio, D.; Chiodo, F.; Reina, J. J.; Marelli, M.; Penades, S.; van Kooyk, Y.; Garcia-Vallejo, J. J.; Bernardi, A., "Effective Targeting of DC-SIGN by $\alpha$ Fucosylamide Functionalized Gold Nanoparticles," Bioconjugate Chem. 2014, $25,2244-2251$.

25. Verez-Bencomo, V.; Fernandez-Santana, V.; Hardy, E.; Toledo, M. E.; Rodríguez, M. C.; Heynngnezz, L.; Rodriguez, A.; Baly, A.; Herrera, L.; Izquierdo, M., "A Synthetic Conjugate Polysaccharide Vaccine Against Haemophilus influenzae Type b," Science 2004, 305, 522-525.

26. Ju, T.; Wang, Y.; Aryal, R. P.; Lehoux, S. D.; Ding, X.; Kudelka, M. R.; Cutler, C.; Zeng, J.; Wang, J.; Sun, X.; Heimburg-Molinaro, J.; Smith, D. F.; Cummings, R. D., "Tn and Sialyl-Tn Antigens, Aberrant O-glycomics as Human Disease Markers," Proteom. Clin. Appl. 2013, 7, 618-631.

27. Sames, D.; Chen, X.-T.; Danishefsky, S. J., "Convergent Total Synthesis of a Tumour-Associated Mucin Motif," Nature 1997, 389, 587-591.

28. Marcaurelle, L. A.; Shin, Y.; Goon, S.; Bertozzi, C. R., "Synthesis of OximeLinked Mucin Mimics Containing the Tumor-Related TN and Sialyl TN Antigens," Org. Lett. 2001, 3, 3691-3694.

29. Wang, P.; Dong, S.; Shieh, J.-H.; Peguero, E.; Hendrickson, R.; Moore, M. A.; Danishefsky, S. J., "Erythropoietin Derived by Chemical Synthesis," Science 2013, 342, 1357-1360.

30. Fais, M.; Karamanska, R.; Allman, S.; Fairhurst, S. A.; Innocenti, P.; Fairbanks, A. J.; Donohoe, T. J.; Davis, B. G.; Russell, D. A.; Field, R. A., "Surface Plasmon Resonance Imaging of Glycoarrays Identifies Novel and Unnatural CarbohydrateBased Ligands for Potential Ricin Sensor Development," Chem. Sci. 2011, 2, 1952-1959.

31. Seeberger, P. H.; Werz, D. B., "Automated Synthesis of Oligosaccharides as a Basis for Drug Discovery," Nat. Rev. Drug. Discov. 2005, 4, 751-763.

32. Chen, W. C.; Completo, G. C.; Sigal, D. S.; Crocker, P. R.; Saven, A.; Paulson, J. C., "In Vivo Targeting of B-Cell Lymphoma with Glycan Ligands of CD22," Blood 2010, 115, 4778-4786.

33. Nycholat, C. M.; Rademacher, C.; Kawasaki, N.; Paulson, J. C., "In Silico-Aided Design of a Glycan Ligand of Sialoadhesin for in Vivo Targeting of Macrophages," J. Am. Chem. Soc. 2012, 134, 15696-15699.

34. Paulick, M. G.; Forstner, M. B.; Groves, J. T.; Bertozzi, C. R., "A Chemical Approach to Unraveling the Biological Function of the Glycosylphosphatidylinositol Anchor," P. Natl. Acad. Sci. 2007, 104, 2033220337. 
35. Ichikawa, M.; Ichikawa, Y., "A Mechanism-Based Affinity-Labeling Agent for Possible use in Isolating N-acetylglucosaminidase," Bioorg. Med. Chem. Lett. 2001, 11, 1769-1773.

36. Song, X.; Xia, B.; Stowell, S. R.; Lasanajak, Y.; Smith, D. F.; Cummings, R. D., "Novel Fluorescent Glycan Microarray Strategy Reveals Ligands for Galectins," Chem. Biol. 2009, 16, 36-47.

37. Likhosherstov, L. M.; Novikova, O. S.; Derevitskaja, V. A.; Kochetkov, N. K., "A New Simple Synthesis of Amino Sugar $\beta$-D-Glycosylamines," Carbohydr. Res. 1986, 146, C1-C5.

38. Kwase, Y. A.; Cochran, M.; Nitz, M., Protecting - Group - Free Glycoconjugate Synthesis: Hydrazide and Oxyamine Derivatives in N - Glycoside Formation. In Modern Synthetic Methods in Carbohydrate Chemistry: From Monosaccharides to Complex Glycoconjugates, Werz, D. B.; Vidal, S., Eds. Wiley-VCH Verlag GmbH \& Co. KGaA: Weinheim, Germany, 2013; pp 67-96.

39. Baues, R. J.; Gray, G. R., "Lectin Purification on Affinity Columns Containing Reductively Aminated Disaccharides," J. Biol. Chem. 1977, 252, 57-60.

40. Gildersleeve, J. C.; Oyelaran, O.; Simpson, J. T.; Allred, B., "Improved Procedure for Direct Coupling of Carbohydrates to Proteins via Reductive Amination," Bioconjugate Chem. 2008, 19, 1485-1490.

41. Urge, L.; Otvos, L.; Lang, E.; Wroblewski, K.; Laczko, I.; Hollosi, M., "FmocProtected, Glycosylated Asparagines Potentially Useful as Reagents in the SolidPhase Synthesis of N-Glycopeptides," Carbohydr. Res. 1992, 235, 83-93.

42. Danishefsky, S. J.; Hu, S.; Cirillo, P. F.; Eckhardt, M.; Seeberger, P. H., "A Highly Convergent Total Synthetic Route to Glycopeptides Carrying a High - Mannose Core Pentasaccharide Domain N - linked to a Natural Peptide Motif," Chem. Eur. J. 1997, 3, 1617-1628.

43. Gudmundsdottir, A. V.; Paul, C. E.; Nitz, M., "Stability Studies of Hydrazide and Hydroxylamine-Based Glycoconjugates in Aqueous Solution," Carbohydr. Res. 2009, 344, 278-284.

44. Leteux, C.; Childs, R. A.; Chai, W.; Stoll, M. S.; Kogelberg, H.; Feizi, T., "Biotinyl-L-3-(2-naphthyl)-alanine Hydrazide Derivatives of N-glycans: Versatile Solid-phase Probes for Carbohydrate-Recognition Studies," Glycobiology 1998, 8, 227-236.

45. Peluso, S.; Imperiali, B., "Asparagine Surrogates for the Assembly of N-linked Glycopeptide Mimetics by Chemoselective Ligation," Tetrahedron Lett. 2001, 42, 2085-2087.

46. Peluso, S.; de L. Ufret, M.; O'Reilly, M. K.; Imperiali, B., "Neoglycopeptides as Inhibitors of Oligosaccharyl Transferase: Insight into Negotiating Product Inhibition," Chem. Biol. 2002, 9, 1323-1328.

47. Compton, J.; Wolfrom, M., "Ring Opening of Galactose Acetates," J. Am. Chem. Soc. 1934, 56, 1157-1162.

48. Peri, F.; Dumy, P.; Mutter, M., "Chemo-and Stereoselective Glycosylation of Hydroxylamino Derivatives: a Versatile Approach to Glycoconjugates," Tetrahedron 1998, 54, 12269-12278.

49. Jimenez-Castells, C.; de la Torre, B. G.; Andreu, D.; Gutierrez-Gallego, R., "Neoglycopeptides: the Importance of Sugar Core Conformation in Oxime-Linked Glycoprobes for Interaction Studies," Glycoconj. J. 2008, 25, 879-887.

50. Langenhan, J. M.; Endo, M. M.; Engle, J. M.; Fukumoto, L. L.; Rogalsky, D. R.; Slevin, L. K.; Fay, L. R.; Lucker, R. W.; Rohlfing, J. R.; Smith, K. R.; Tjaden, A. 
E.; Werner, H. M., "Synthesis and Biological Evaluation of RON-Neoglycosides as Tumor Cytotoxins," Carbohydr. Res. 2011, 346, 2663-2676.

51. Bohorov, O.; Andersson-Sand, H.; Hoffmann, J.; Blixt, O., "Arraying Glycomics: a Novel Bi-functional Spacer for One-step Microscale Derivatization of Free Reducing Glycans," Glycobiology 2006, 16, 21C-27C.

52. Prudden, A. R.; Chinoy, Z. S.; Wolfert, M. A.; Boons, G.-J., "A Multifunctional Anomeric Linker for the Chemoenzymatic Synthesis of Complex Oligosaccharides," Chem. Commun. 2014, 50, 7132-7135.

53. Munneke, S.; Prevost, J. R.; Painter, G. F.; Stocker, B. L.; Timmer, M. S., "The Rapid and Facile Synthesis of Oxyamine Linkers for the Preparation of Hydrolytically Stable Glycoconjugates," Org. Lett. 2015, 17, 624-627.

54. Carrasco, M. R.; Nguyen, M. J.; Burnell, D. R.; MacLaren, M. D.; Hengel, S. M., "Synthesis of Neoglycopeptides by Chemoselective Reaction of Carbohydrates with Peptides Containing a Novel N'-methyl-aminooxy Amino Acid," Tetrahedron Lett. 2002, 43, 5727-5729.

55. Carrasco, M. R.; Brown, R. T., "A Versatile set of Aminooxy Amino Acids for the Synthesis of Neoglycopeptides," J. Org. Chem. 2003, 68, 8853-8858.

56. Peri, F.; Jiménez-Barbero, J.; García-Aparicio, V.; Tvaroška, I.; Nicotra, F., "Synthesis and Conformational Analysis of Novel $\mathrm{N}-\left(\mathrm{OCH}_{3}\right)$ - linked Disaccharide Analogues," Chem. Eur. J. 2004, 10, 1433-1444.

57. Peri, F.; Deutman, A.; Ferla, B. L.; Nicotra, F., "Solution and Solid-Phase Chemoselective Synthesis of (1-6)-Amino(methoxy) Di- and Trisaccharide Analogues," Chem. Commun. 2002, 1504-1505.

58. Langenhan, J. M.; Griffith, B. R.; Thorson, J. S., "Neoglycorandomization and Chemoenzymatic Glycorandomization: Two Complementary Tools for Natural Product Diversification," J. Nat. Prod. 2005, 68, 1696-1711.

59. Langenhan, J. M.; Peters, N. R.; Guzei, I. A.; Hoffmann, F. M.; Thorson, J. S., "Enhancing the Anticancer Properties of Cardiac Glycosides by Neoglycorandomization," P. Natl. Acad. Sci. 2005, 102, 12305-12310.

60. Goff, R. D.; Thorson, J. S., "Enhancing the Divergent Activities of Betulinic Acid via Neoglycosylation," Org. Lett. 2008, 11, 461-464.

61. Goff, R. D.; Thorson, J. S., "Enhancement of Cyclopamine via Conjugation with Nonmetabolic Sugars," Org. Lett. 2012, 14, 2454-2457.

62. Peltier - Pain, P.; Timmons, S. C.; Grandemange, A.; Benoit, E.; Thorson, J. S., "Warfarin Glycosylation Invokes a Switch from Anticoagulant to Anticancer Activity," ChemMedChem 2011, 6, 1347-1350.

63. Griffith, B. R.; Krepel, C.; Fu, X.; Blanchard, S.; Ahmed, A.; Edmiston, C. E.; Thorson, J. S., "Model for Antibiotic Optimization via Neoglycosylation: Synthesis of Liponeoglycopeptides Active Against VRE," J. Am. Chem. Soc. 2007, 129, 8150-8155.

64. Ahmed, A.; Peters, N. R.; Fitzgerald, M. K.; Watson, J. A., Jr.; Hoffmann, F. M.; Thorson, J. S., "Colchicine Glycorandomization Influences Cytotoxicity and Mechanism of Action," J. Am. Chem. Soc. 2006, 128, 14224-14225.

65. Goff, R. D.; Thorson, J. S., "Neoglycosylation and Neoglycorandomization: Enabling Tools for the Discovery of Novel Glycosylated Bioactive Probes and Early Stage Leads," Medchemcomm 2014, 5, 1036-1047.

66. Cló, E.; Blixt, O.; Jensen, K. J., "Chemoselective Reagents for Covalent Capture and Display of Glycans in Microarrays," Eur. J. Org. Chem. 2010, 2010, 540-554. 
67. Araujo, A. C.; Souto-Padrón, T.; de Souza, W., "Cytochemical Localization of Carbohydrate Residues in Microfilariae of Wuchereria bancrofti and Brugia malayi," J. Histochem. Cytochem. 1993, 41, 571-578.

68. Fuhrman, J. A.; Piessens, W. F., "Chitin Synthesis and Sheath Morphogenesis in Brugia malayi Microfilariae," Mol. Biochem. Parasitol. 1985, 17, 93-104.

69. Neville, A. C.; Parry, D. A.; Woodhead-Galloway, J., "The Chitin Crystallite in Arthropod Cuticle," J. Cell Sci. 1976, 21, 73-82.

70. Shahabuddin, M.; Kaslow, D. C., "Plasmodium: Parasite Chitinase and Its Role in Malaria Transmission," Exp. Parasitol. 1994, 79, 85-88.

71. Shibata, Y.; Metzger, W. J.; Myrvik, Q. N., "Chitin Particle-Induced CellMediated Immunity is Inhibited by Soluble Mannan: Mannose ReceptorMediated Phagocytosis Initiates IL-12 Production.," J. Immunol. 1997, 159, 24622467.

72. Da Silva, C. A.; Hartl, D.; Liu, W.; Lee, C. G.; Elias, J. A., "TLR-2 and IL-17A in Chitin-Induced Macrophage Activation and Acute Inflammation," J. Immunol. 2008, 181, 4279-4286.

73. Shibata, Y.; Foster, L. A.; Metzger, W. J.; Myrvik, Q. N., "Alveolar Macrophage Priming by Intravenous Administration of Chitin Particles, Polymers of N-acetylD-glucosamine, in Mice," Infect. Immun. 1997, 65, 1734-1741.

74. Shibata, Y.; Foster, L. A.; Bradfield, J. F.; Myrvik, Q. N., "Oral Administration of Chitin Down-Regulates Serum IgE Levels and Lung Eosinophilia in the Allergic Mouse," J. Immunol. 2000, 164, 1314-1321.

75. Reese, T. A.; Liang, H.-E.; Tager, A. M.; Luster, A. D.; Van Rooijen, N.; Voehringer, D.; Locksley, R. M., "Chitin Induces Accumulation in Tissue of Innate Immune Cells Associated with Allergy," Nature 2007, 447, 92-96.

76. Da Silva, C. A.; Pochard, P.; Lee, C. G.; Elias, J. A., "Chitin Particles are Multifaceted Immune Adjuvants," Am. J. Respir. Crit. Care Med. 2010, 182, 1482-1491.

77. Murphy, K., Janeways Immunobiology. 8 ed.; Garland Science: New York, 2011.

78. Da Silva, C. A.; Chalouni, C.; Williams, A.; Hartl, D.; Lee, C. G.; Elias, J. A., "Chitin Is a Size-Dependent Regulator of Macrophage TNF and IL-10 Production," J. Immunol. 2009, 182, 3573-3582.

79. Lee, C. G.; Da Silva, C. A.; Lee, J.-Y.; Hartl, D.; Elias, J. A., "Chitin Regulation of Immune Responses: An Old Molecule With New Roles," Curr. Opin. Immunol. 2008, 20, 684-689.

80. Van Dyken, S. J.; Garcia, D.; Porter, P.; Huang, X.; Quinlan, P. J.; Blanc, P. D.; Corry, D. B.; Locksley, R. M., "Fungal Chitin from Asthma-Associated Home Environments Induces Eosinophilic Lung Infiltration," J. Immunol. 2011, 187, 2261-2267.

81. Marradi, M.; Chiodo, F.; Garcia, I.; Penades, S., "Glyconanoparticles as Multifunctional and Multimodal Carbohydrate Systems," Chem. Soc. Rev. 2013, $42,4728-4745$.

82. Arvizo, R.; Bhattacharya, R.; Mukherjee, P., "Gold Nanoparticles: Opportunities and Challenges in Nanomedicine," Expert Opin. Drug Deliv. 2010, 7, 753-763.

83. Mei, B. C.; Oh, E.; Susumu, K.; Farrell, D.; Mountziaris, T. J.; Mattoussi, H., "Effects of Ligand Coordination Number and Surface Curvature on the Stability of Gold Nanoparticles in Aqueous Solutions," Langmuir 2009, 25, 10604-10611.

84. Boisselier, E.; Astruc, D., "Gold Nanoparticles in Nanomedicine: Preparations, Imaging, Diagnostics, Therapies and Toxicity," Chem. Soc. Rev. 2009, 38, 17591782. 
85. van Kooyk, Y.; Rabinovich, G. A., "Protein-Glycan Interactions in the Control of Innate and Adaptive Immune Responses," Nat. Immunol. 2008, 9, 593-601.

86. Spiro, R. G., "Protein Glycosylation: Nature, Distribution, Enzymatic Formation, and Disease Implications of Glycopeptide Bonds," Glycobiology 2002, 12, 43R56R.

87. Clayden, J.; Greeves, N.; Warren, S. G., Organic Chemistry. Oxford University Press: Oxford; New YorK, 2012.

88. Gudmundsdottir, A. V.; Nitz, M., "Hydrolysis Rates of 1-glucosyl-2benzoylhydrazines in Aqueous Solution," Carbohydr. Res. 2007, 342, 749-752.

89. Munneke, S. Novel Methodologies for the Synthesis of Multivalent Glycoconjugates. PhD, Victoria University of Wellington, Wellington, New Zealand, 2015.

90. "Wavelength Index", retrieved from http://www.sigmaaldrich.com/lifescience/cell-biology/detection/learning-center/wavelength-index.html, last accessed (15 January 2017).

91. Bordwell, F. G., "Equilibrium Acidities in Dimethyl Sulfoxide Solution," Acc. Chem.Res. 1988, 21, 456-463.

92. Swarts, F., "Sur l'acide Trifluoracétique," Bull. Acad. R. Belg. 1922, 8, 343-370.

93. Timell, T. E.; Enterman, W.; Spencer, F.; Soltes, E. J., "The Acid Hydrolysis of Glycosides: II. Effect of Substituents at C-5," Can. J. Chem. 1965, 43, 2296-2305.

94. Jencks, W. P., "Mechanism and Catalysis of Simple Carbonyl Group Reactions," Prog. Phys. Org. Chem. 1964, 2, 63.

95. Kwan, E. E., "Factors Affecting the Relative Efficiency of General Acid Catalysis," J. Chem. Educ. 2005, 82, 1026.

96. Ault, A., "General Acid and General Base Catalysis," J. Chem. Educ. 2007, 84, 38.

97. Reijenga, J.; van Hoof, A.; van Loon, A.; Teunissen, B., "Development of Methods for the Determination of $\mathrm{p} K_{\mathrm{a}}$ Values," Anal. Chem. Insights 2013, 8, 5371.

98. Flash, P., "Small Scale Determination of the $\mathrm{p} K_{\mathrm{a}}$ Values for Organic Acids," $J$. Chem. Educ. 1994, 71, A6.

99. Stephens, S. J.; Jonich, M. J., "Determination of $\mathrm{p} K_{\mathrm{a}}$ Using the Half-Volume Method: A Laboratory Experiment," J. Chem. Educ. 1977, 54, 711.

100. Lopalco, A.; Douglas, J.; Denora, N.; Stella, V. J., "Determination of $\mathrm{p} K_{\mathrm{a}}$ and Hydration Constants for a Series of $\alpha$-Keto-Carboxylic Acids Using Nuclear Magnetic Resonance Spectrometry," J. Pharm. Sci. 2016, 105, 664-672.

101. Handloser, C. S.; Chakrabarty, M. R.; Mosher, M. W., "Experimental Determination of $\mathrm{p} K_{\mathrm{a}}$ Values by use of NMR Chemical Shift," J. Chem. Educ. 1973, 50, 510 .

102. Mohr, C. F., "Neue Massanalytische Bestimmung des Chlors in Verbindungen," Ann. Chem. Pharm. 1856, 97, 335-338.

103. Macindoe, W. M.; van Oijen, A. H.; Boons, G.-J., "A Unique and Highly Facile Method for Synthesising Disulfide Linked Neoglycoconjugates: a New Approach for Remodelling of Peptides and Proteins," Chem. Commun. 1998, 847-848.

104. Pensa, E.; Cortés, E.; Corthey, G.; Carro, P.; Vericat, C.; Fonticelli, M. H.; Benítez, G.; Rubert, A. A.; Salvarezza, R. C., "The Chemistry of the Sulfur-Gold Interface: In Search of a Unified Model," Acc. Chem. Res. 2012, 45, 1183-1192.

105. Martínez-Ávila, O.; Hijazi, K.; Marradi, M.; Clavel, C.; Campion, C.; Kelly, C.; Penadés, S., "Gold Manno-Glyconanoparticles: Multivalent Systems to Block 
HIV-1 gp120 Binding to the Lectin DC-SIGN," Chem. Eur. J. 2009, 15, 98749888.

106. Mei, B. C.; Susumu, K.; Medintz, I. L.; Mattoussi, H., "Polyethylene GlycolBased Bidentate Ligands to Enhance Quantum Dot and Gold Nanoparticle Stability in Biological Media," Nat. Protoc. 2009, 4, 412-423.

107. Cheng, C.-C.; Huang, Y.-C.; Liu, M.-C., "DNA Hydrolysis Catalyzed by Tris(diisopropyl-1,4,7-triazacyclononanes)ethane Metal Complexes," J. Chin. Chem. Soc. 2004, 51, 1201-1208.

108. Li, Z.; Wu, Z.; Luo, F., "Synthesis and Antifungal Activities of Alkyl N-(1,2,3Thiadiazole-4-Carbonyl) Carbamates and S-Alkyl N-(1,2,3-Thiadiazole-4Carbonyl) Carbamothioates," J. Agric. Food Chem. 2005, 53, 3872-3876.

109. Kim, J. H.; Gallagher, M. J.; Toia, R. F., "Methanolysis of 4-Methyl-2,6,7-trioxa1-phospha-bicyclo[2.2.2]octane 1-Oxide and 1-Sulfide: Mechanistic and Stereochemical Considerations," Aust. J. Chem. 1994, 47, 715-722.

110. Pascal, R. A.; Winans, C. G.; Van Engen, D., "Small, Strained Cyclophanes with Methine Hydrogens Projected Toward the Centers of Aromatic Rings," J. Am. Chem. Soc. 1989, 111, 3007-3010.

111. Horikoshi, H.; Takeuchi, M. The Novel Tetrathia Spiro Compound Showing High Refractive Index, Heat and Impact Resistance. JP 2010018592, 2010.

112. Lin, H.; Zou, Y.; Lv, G.; Pu, J.; Hong, A. Two-Component Epoxy Resin Adhesives Containing Modified Polythiols as Curing Agents and the Preparation Methods Therefor. CN 102086363, 2011.

113. Russavskaya, N. V.; Alekminskaya, O. V.; Deryagina, E. N.; Skotheim, T. A.; Trofimov, B. A., "A New Route to 1,2,3-Propanetrithiol," Russ. J. Gen. Chem. 2001, 71, 1771-1772.

114. Guyon, V.; Guy, A.; Foos, J.; Lemaire, M.; Draye, M., "Lipophilic Polythiamacrocycles as Palladium Extracting Agents," Tetrahedron 1995, 51, 4065-4074.

115. Bottini, A. T.; Böttner, E. F., "Reactions with Base of 2-Propargylthioethanol and the 2-(2-Haloallylthio)ethanols1," J. Org. Chem. 1966, 31, 385-388.

116. Valiulin, R. A.; Kutateladze, A. G., "2,6,7-Trithiabicyclo[2.2.2]octanes as Promising Photolabile Tags for Combinatorial Encoding," J. Org. Chem. 2008, 73, 335-338.

117. Petrovski, Ž.; Romão, C. C.; Afonso, C. A. M., "Synthesis of Tris(N,Ndimethylthiocarbamoyl)-1,1,1-tris-(methylaminomethyl)ethane and its Application as Ligand for Pauson-Khand Reaction," Synth. Commun. 2008, 38, 2761-2767.

118. Wessig, P.; Gerngroß, M.; Freyse, D.; Bruhns, P.; Przezdziak, M.; Schilde, U.; Kelling, A., "Molecular Rods Based on Oligo-spiro-thioketals," J. Org. Chem. 2016, 81, 1125-1136.

119. Min, B.; Prout, M.; Hu-Li, J.; Zhu, J.; Jankovic, D.; Morgan, E. S.; Urban, J. F.; Dvorak, A. M.; Finkelman, F. D.; LeGros, G., "Basophils Produce IL-4 and Accumulate in Tissues After Infection with a Th2-Inducing Parasite," J. Exp. Med. 2004, 200, 507-517. 
Appendix 2

Spectra 


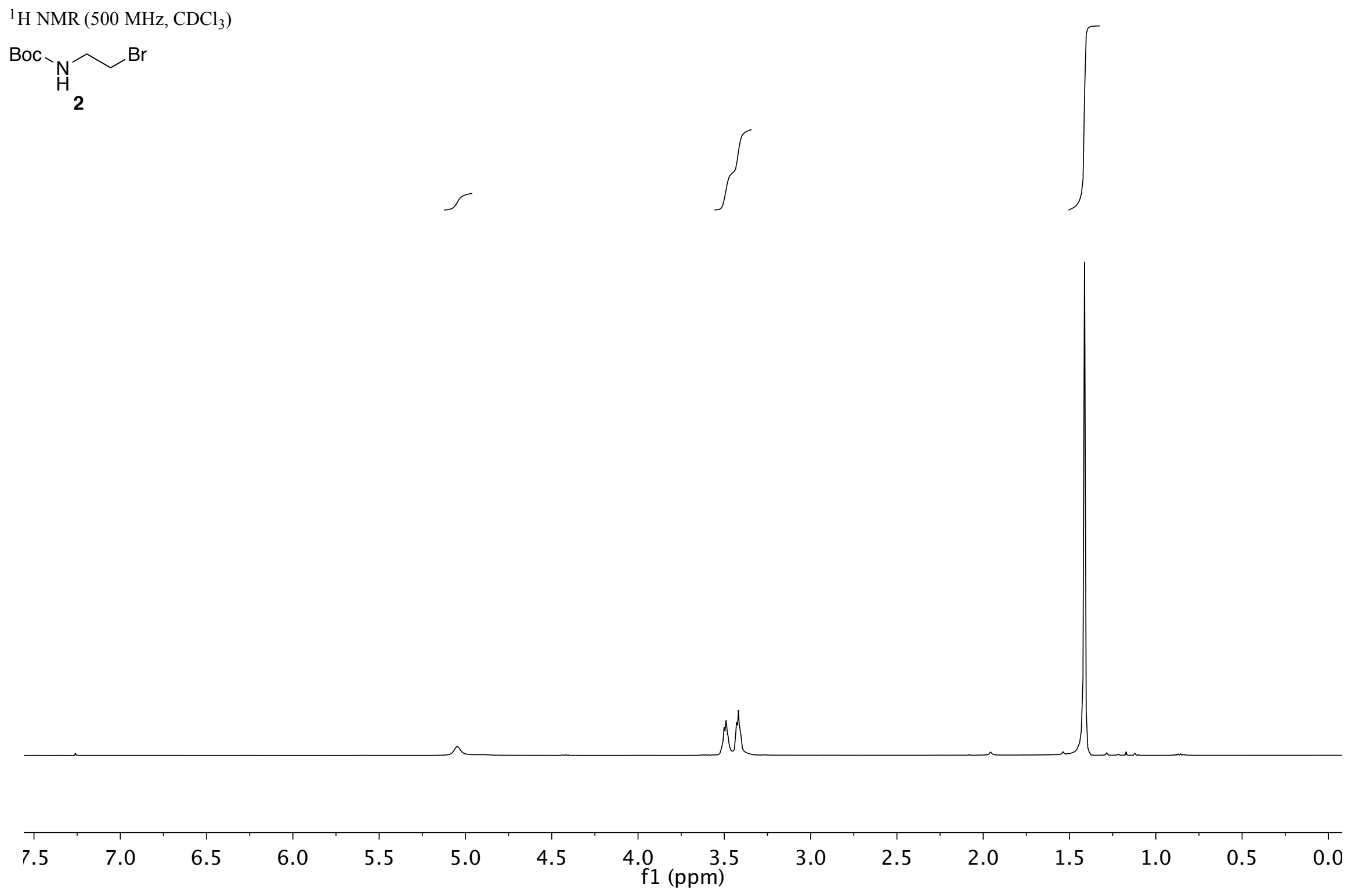


${ }^{13} \mathrm{C}$ NMR (125 MHz, $\mathrm{CDCl}_{3}$ )

Boc $\sim \mathrm{Br}$

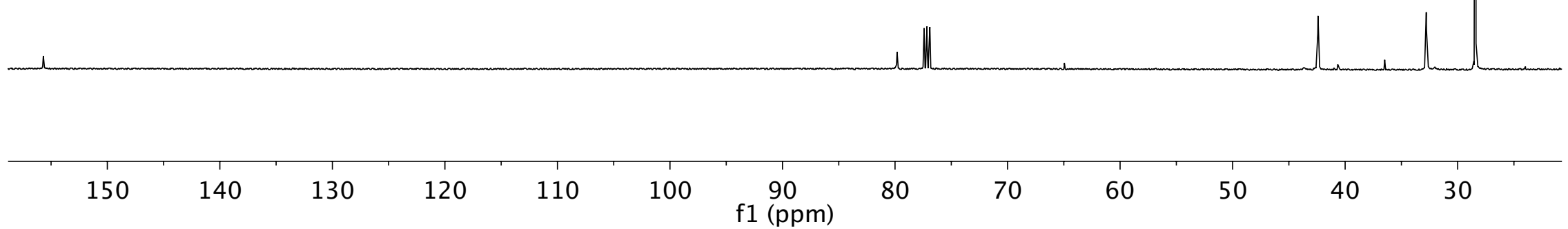




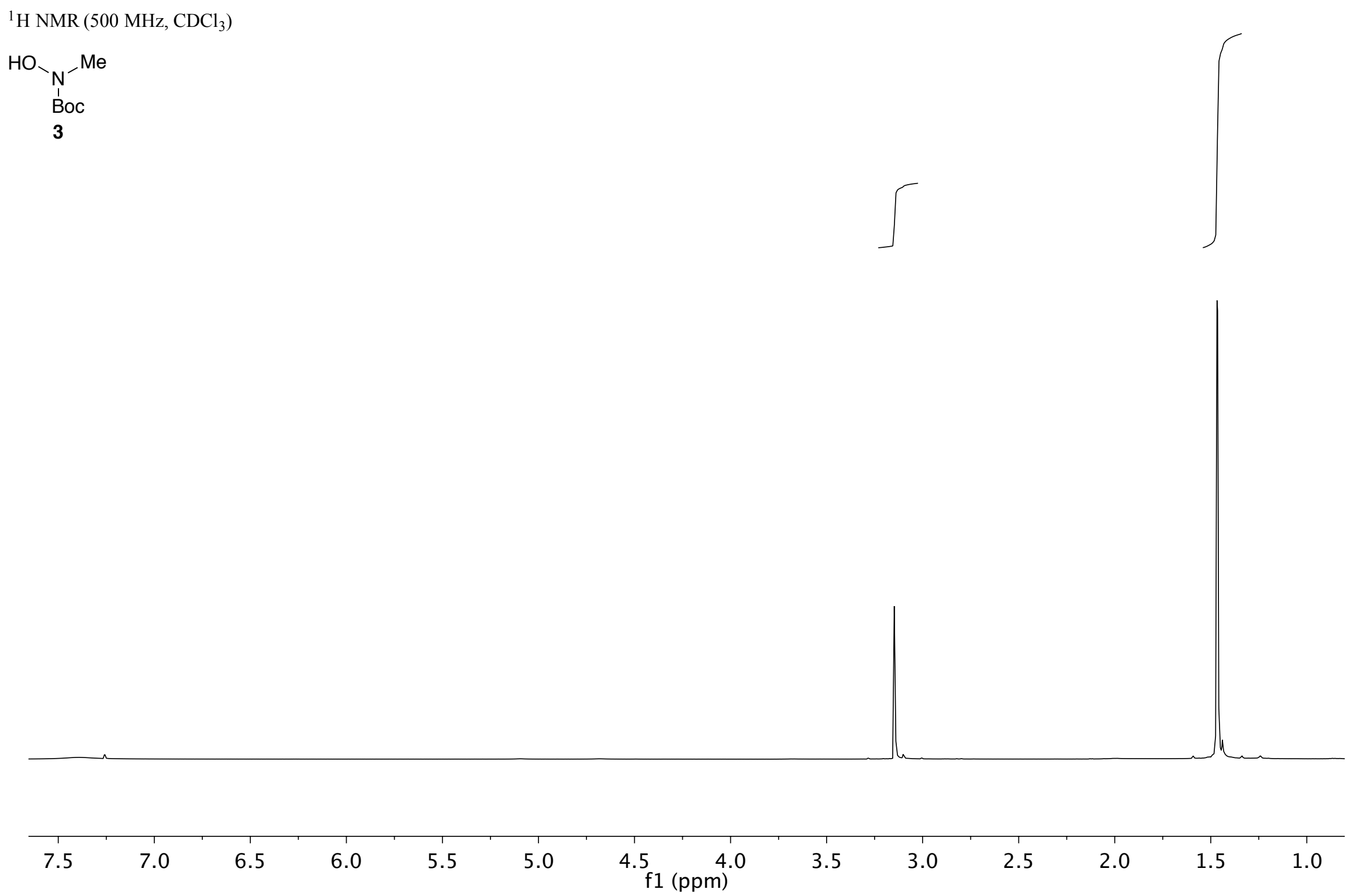


${ }^{13} \mathrm{C}$ NMR $\left(125 \mathrm{MHz}, \mathrm{CDCl}_{3}\right)$

HO ${ }_{\mathrm{N}^{\prime}}-\mathrm{Me}$

3

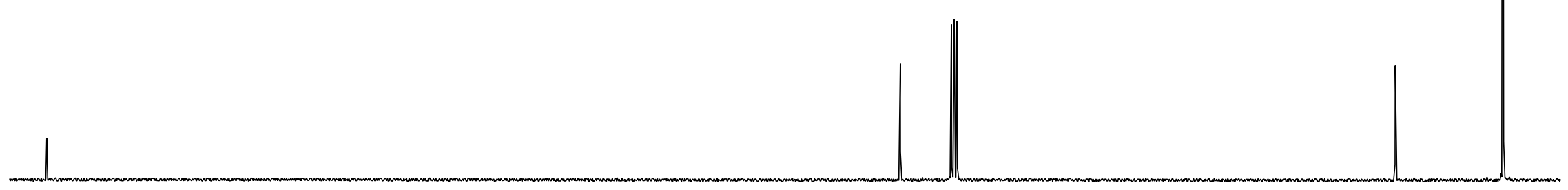

\begin{tabular}{llllll|r|r|}
\hline 60 & 150 & 140 & 130 & 120 & 110 & 100
\end{tabular}

f1 $\begin{gathered}90 \\ \text { ppm) }\end{gathered}$ 
${ }^{1} \mathrm{H} \mathrm{NMR}\left(500 \mathrm{MHz}, \mathrm{CDCl}_{3}\right)$

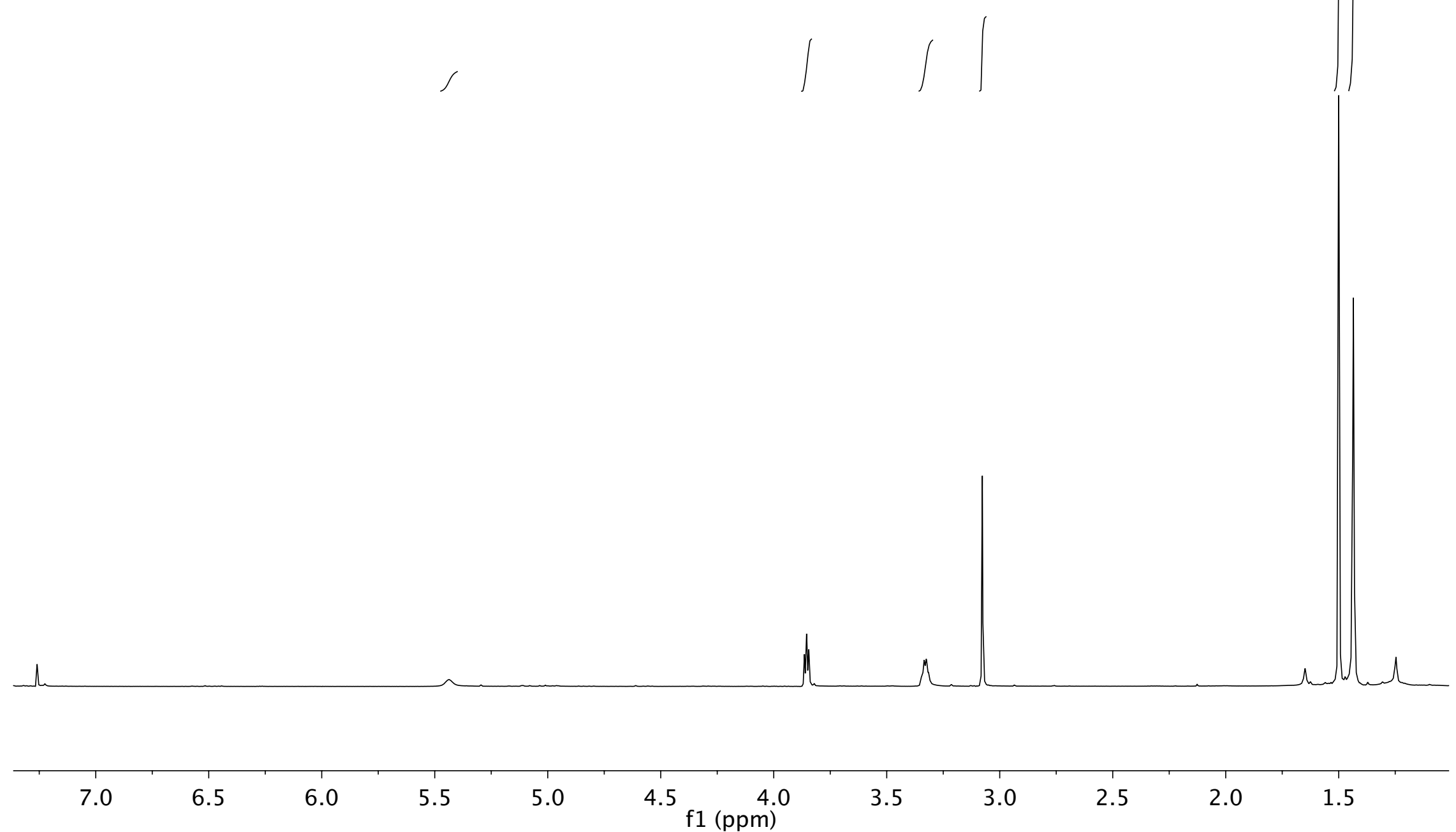


${ }^{13} \mathrm{C}$ NMR $\left(125 \mathrm{MHz}, \mathrm{CDCl}_{3}\right)$

$\mathrm{Boc}^{\mathrm{Me}}{ }_{-}^{\mathrm{N}} \mathrm{O}_{\text {Boc }}^{\mathrm{H}}$

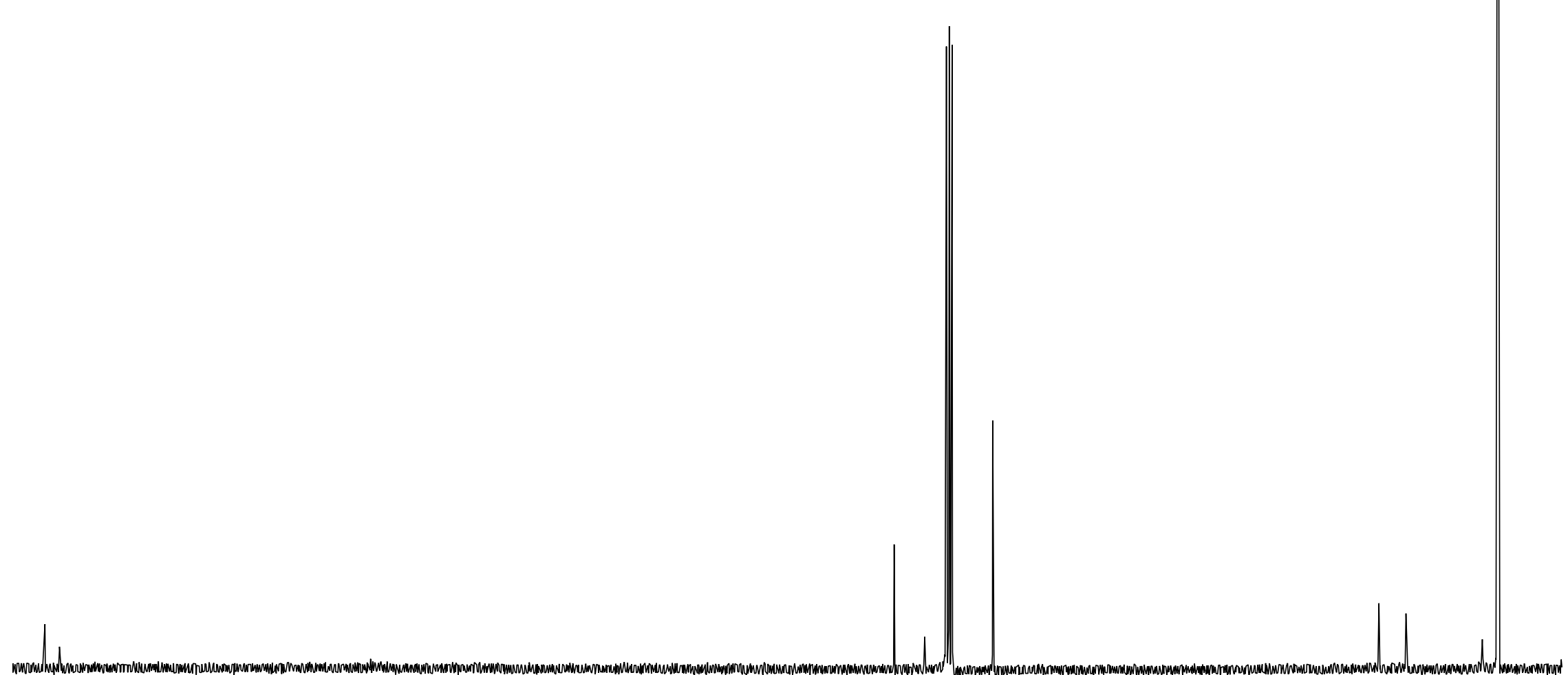

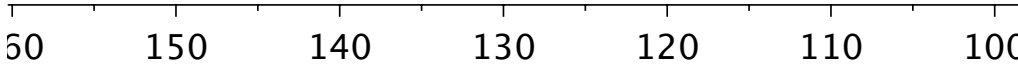

f1 90 
${ }^{1} \mathrm{H}$ NMR (500 MHz, $\mathrm{D}_{2} \mathrm{O}$ )

$\stackrel{\mathrm{Me}}{1} \underset{\mathrm{O}}{ } \sim \mathrm{NH}_{2}$

4
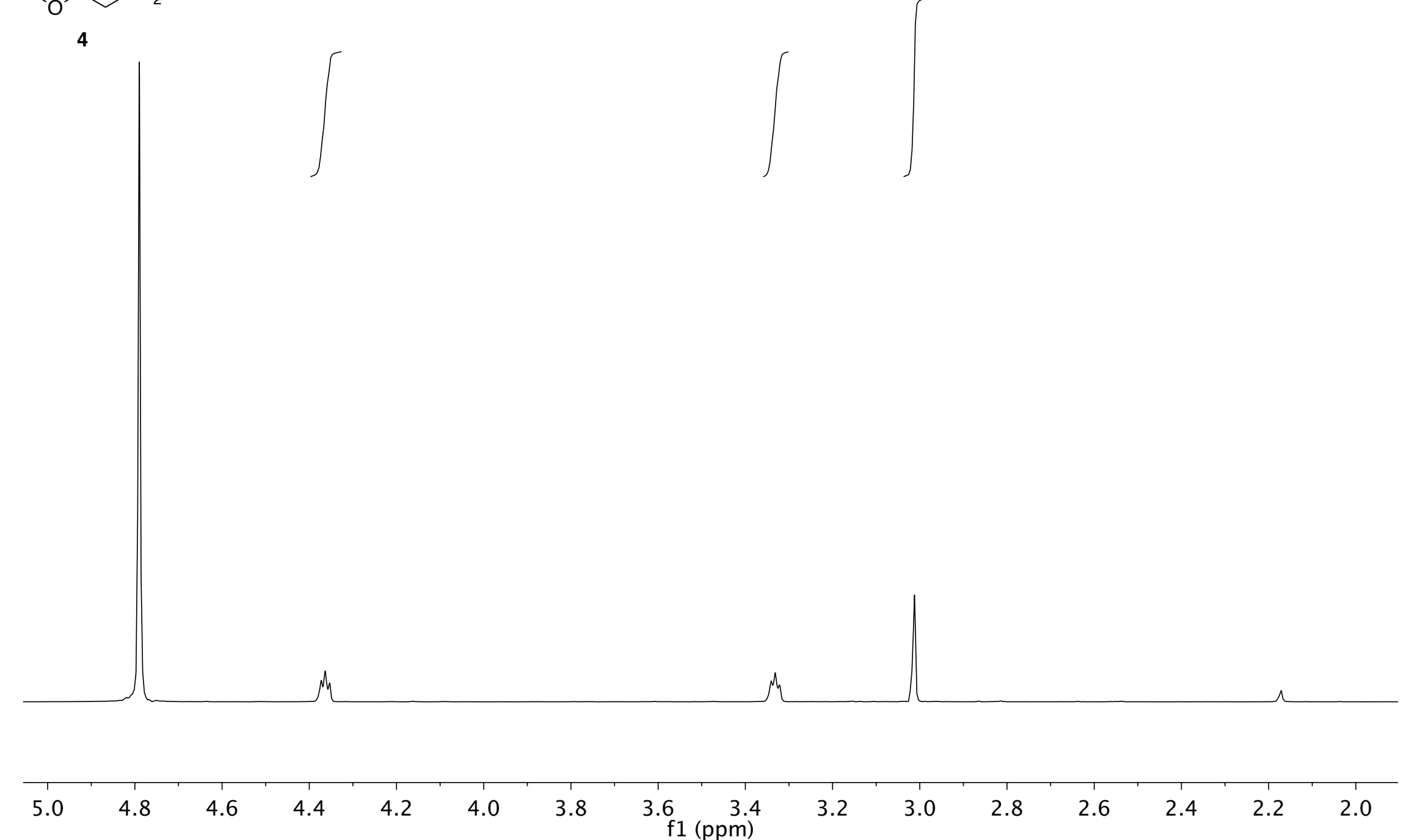
${ }^{13} \mathrm{C}$ NMR (125 MHz, $\left.\mathrm{D}_{2} \mathrm{O}\right)$

$\mathrm{Me}$

$\mathrm{HN}^{\prime} \sim \mathrm{NH}_{2}$

4

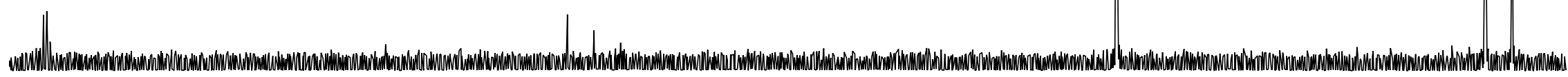

$\begin{array}{lllllllllllllllllllllllllll}165 & 160 & 155 & 150 & 145 & 140 & 135 & 130 & 125 & 120 & 115 & 110 & 105 & 100 & 95 & 90 & 85 & 80 & 75 & 70 & 65 & 60 & 55 & 50 & 45 & 40 & 35\end{array}$

f1 (ppm) 


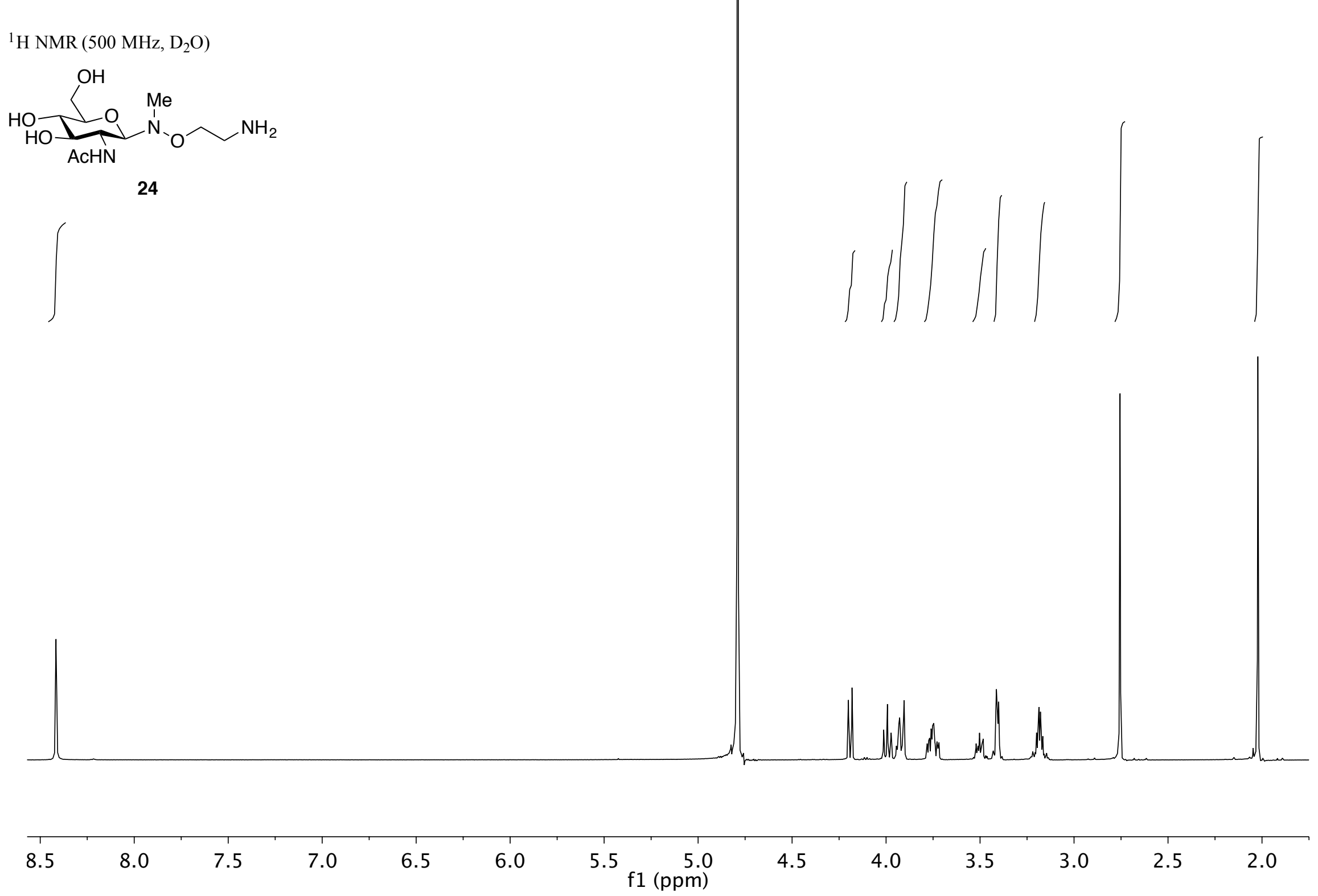


${ }^{13} \mathrm{C}$ NMR (125 MHz, $\mathrm{D}_{2} \mathrm{O}$ )

AcHN

(1)

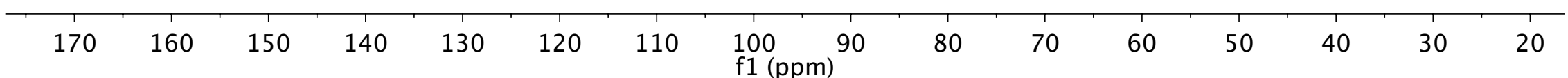



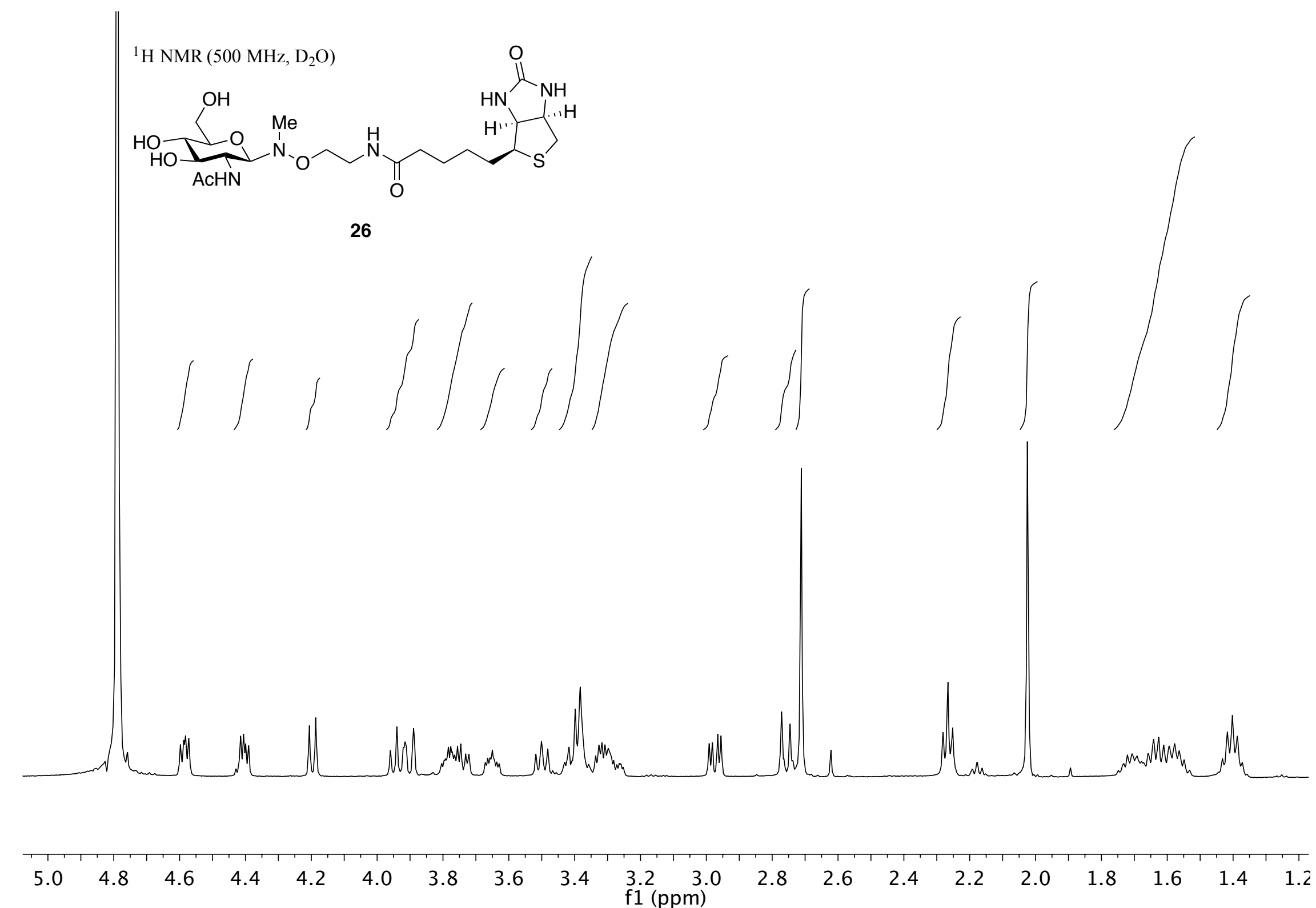


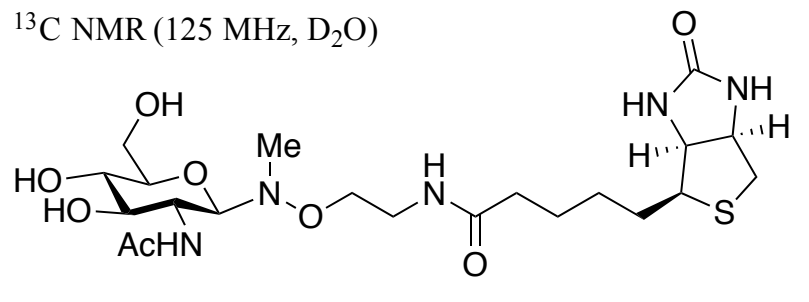

26

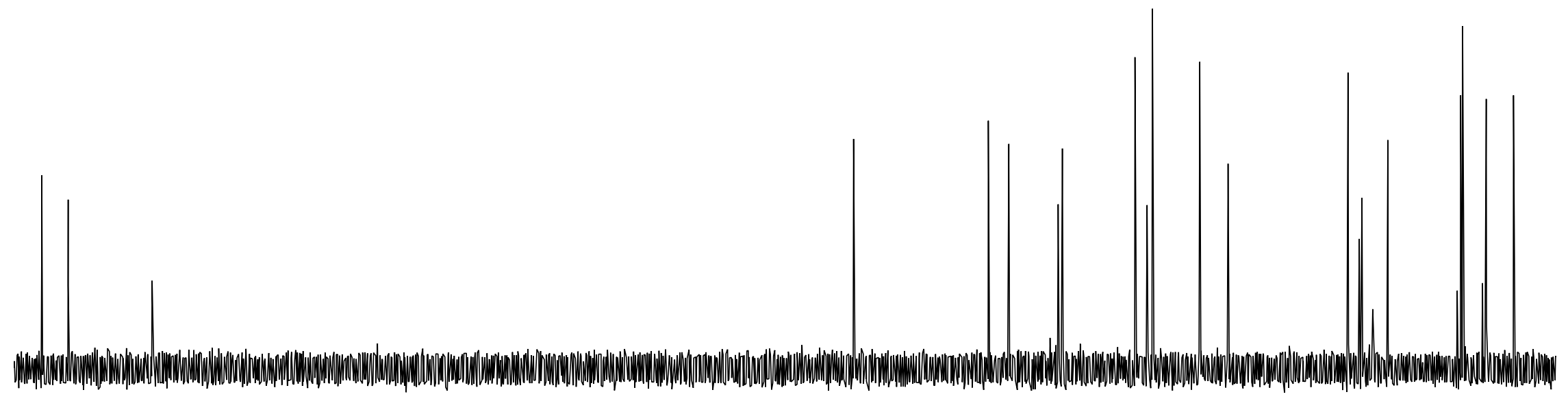

$170 \quad 160$

150

140

130

120

110

100

90

80

70

60

50

40

30

20 
${ }^{1} \mathrm{H}$ NMR (500 MHz, D $2 \mathrm{O}$ )
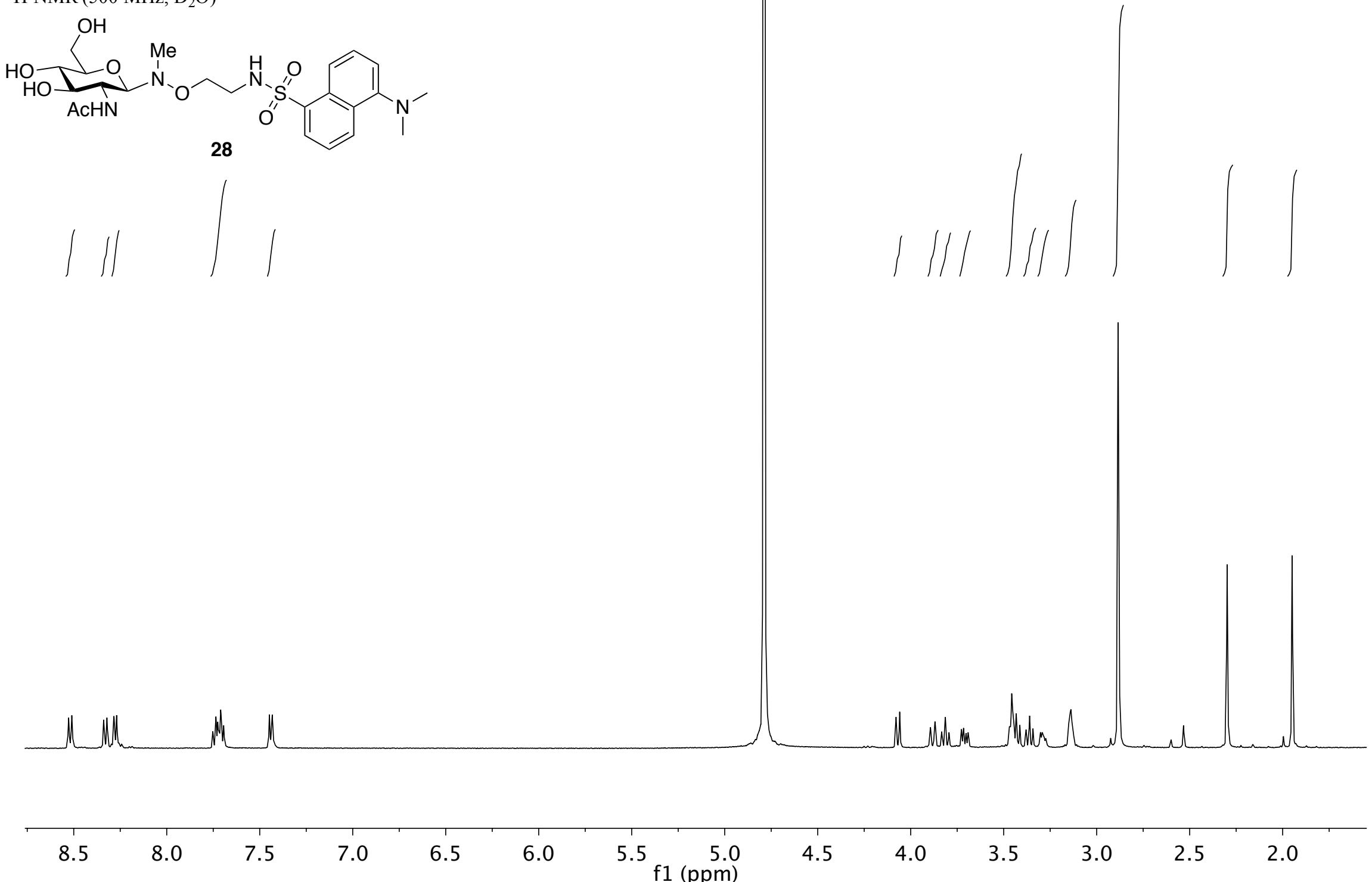
${ }^{13} \mathrm{C}$ NMR (125 MHz, $\mathrm{D}_{2} \mathrm{O}$ )

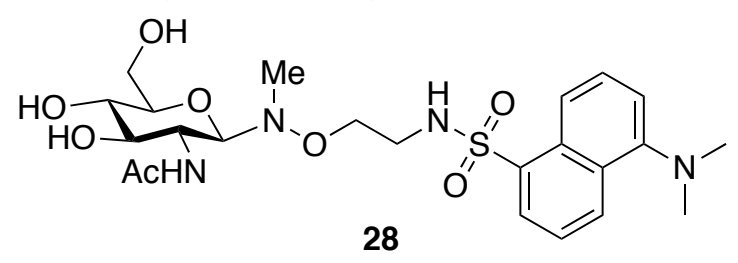

I.w.

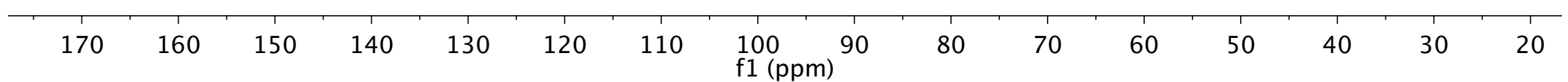



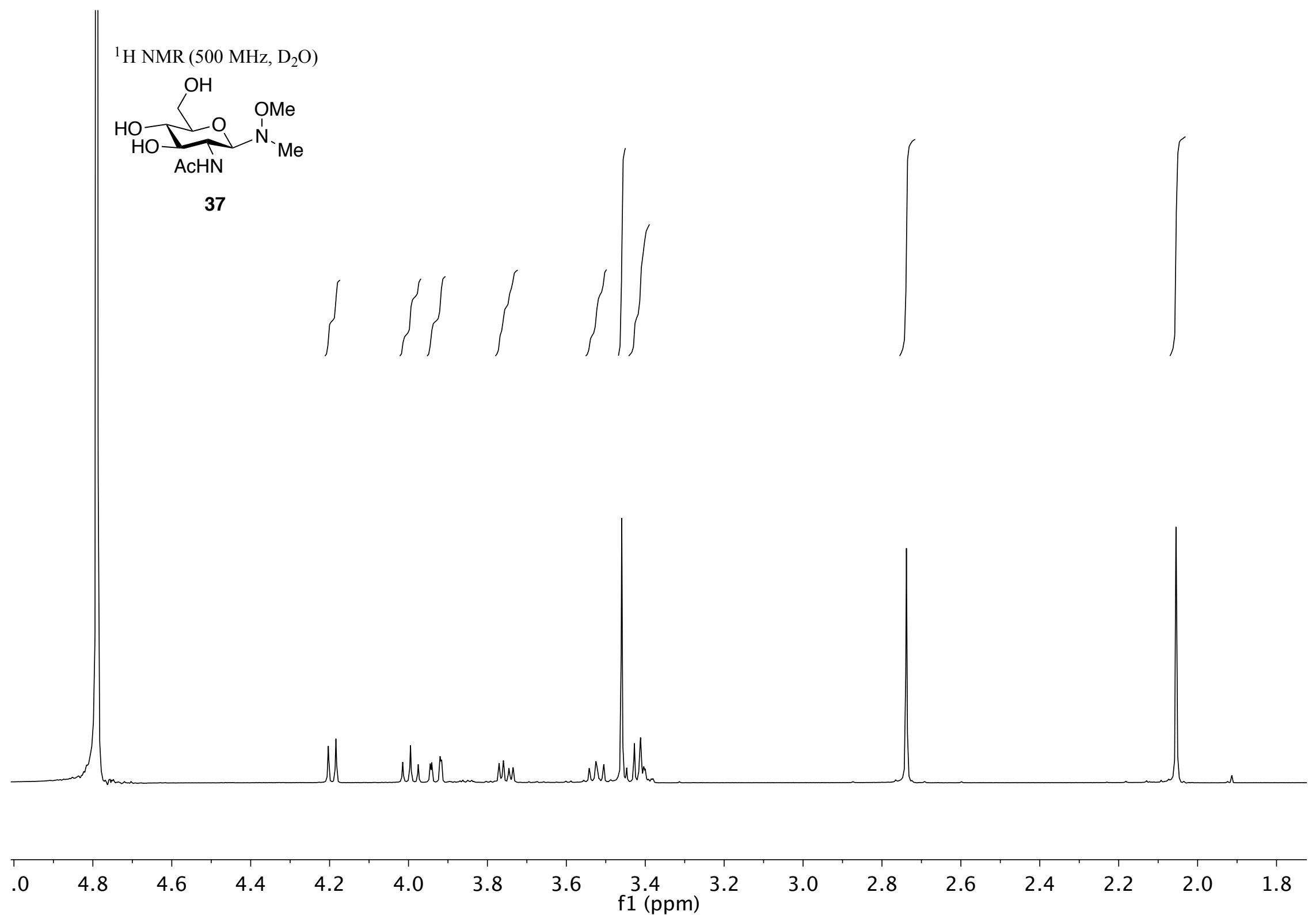
${ }^{13} \mathrm{C}$ NMR (125 MHz, $\mathrm{D}_{2} \mathrm{O}$ )

AcHN

37

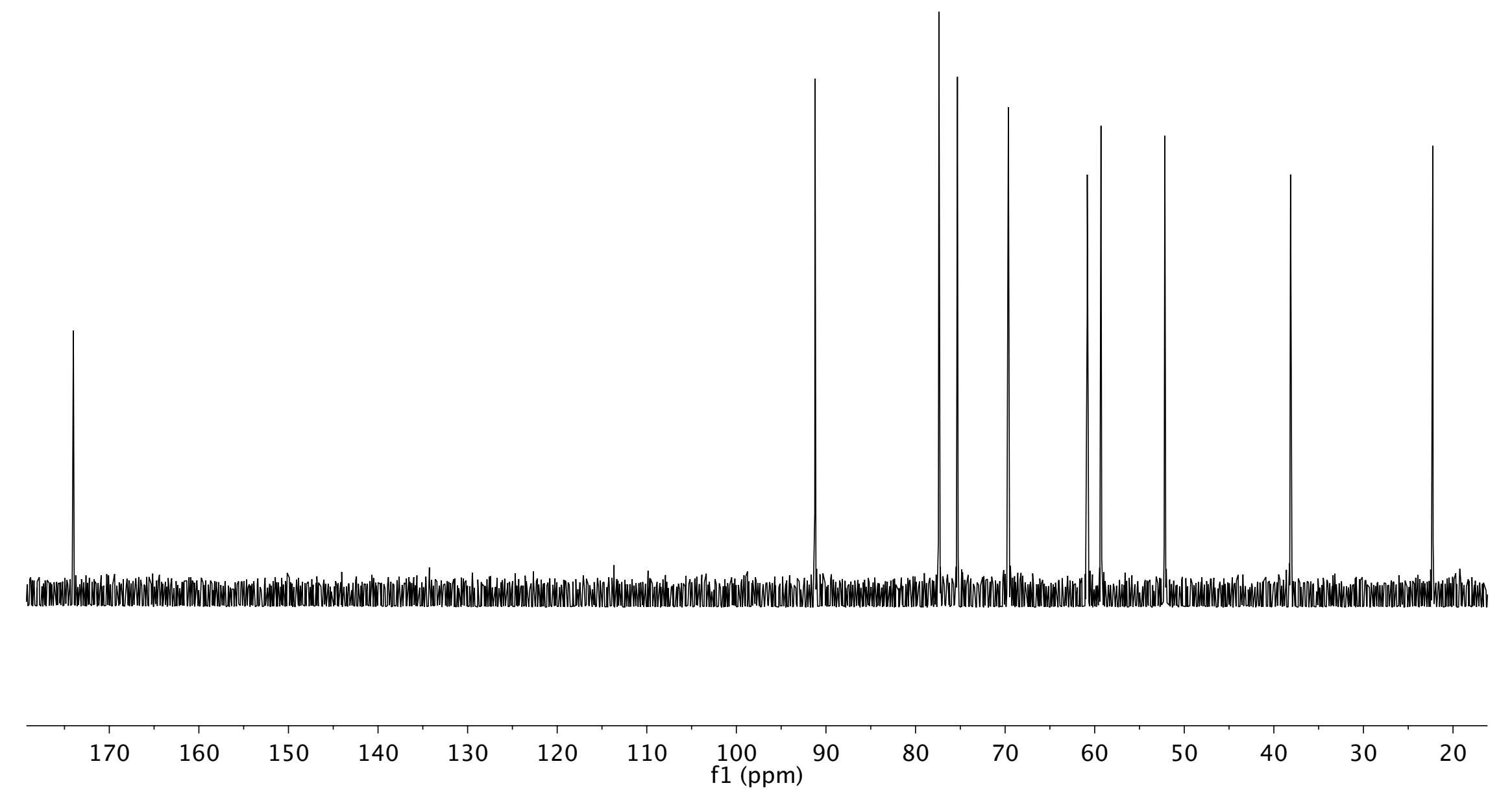


${ }^{1} \mathrm{H}$ NMR (500 MHz, $\mathrm{CDCl}_{3}$ )

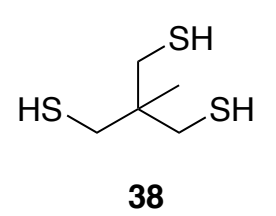

38

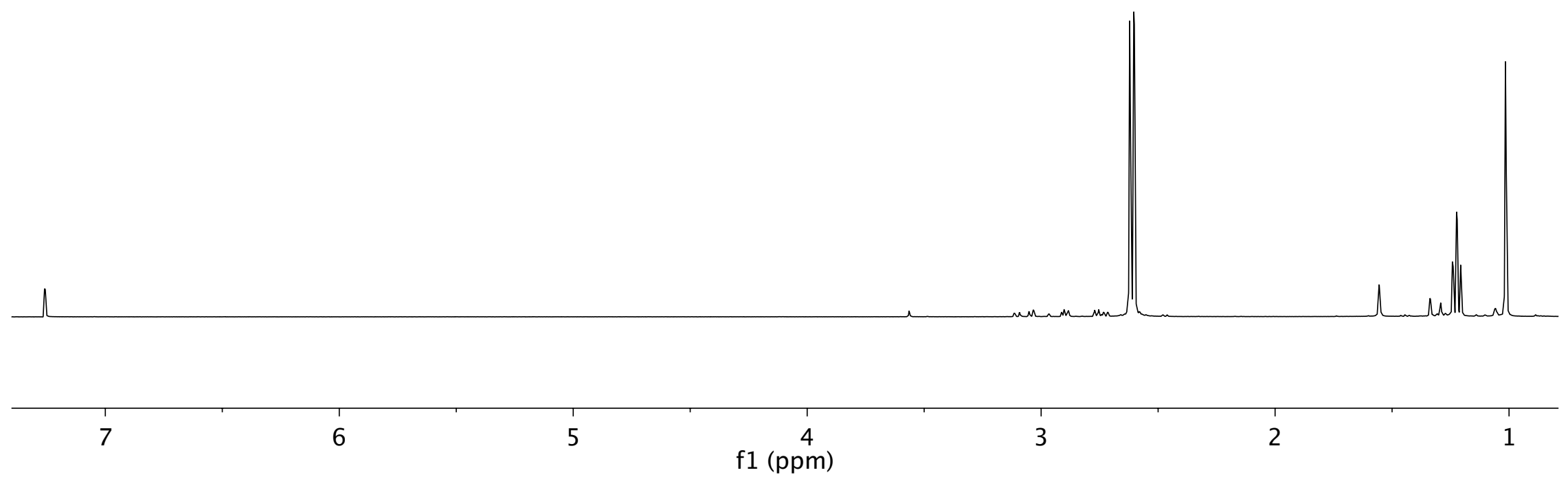


${ }^{13} \mathrm{C}$ NMR $\left(125 \mathrm{MHz}, \mathrm{CDCl}_{3}\right)$

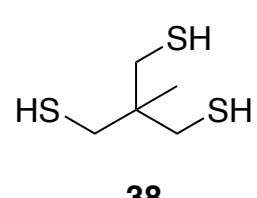

38

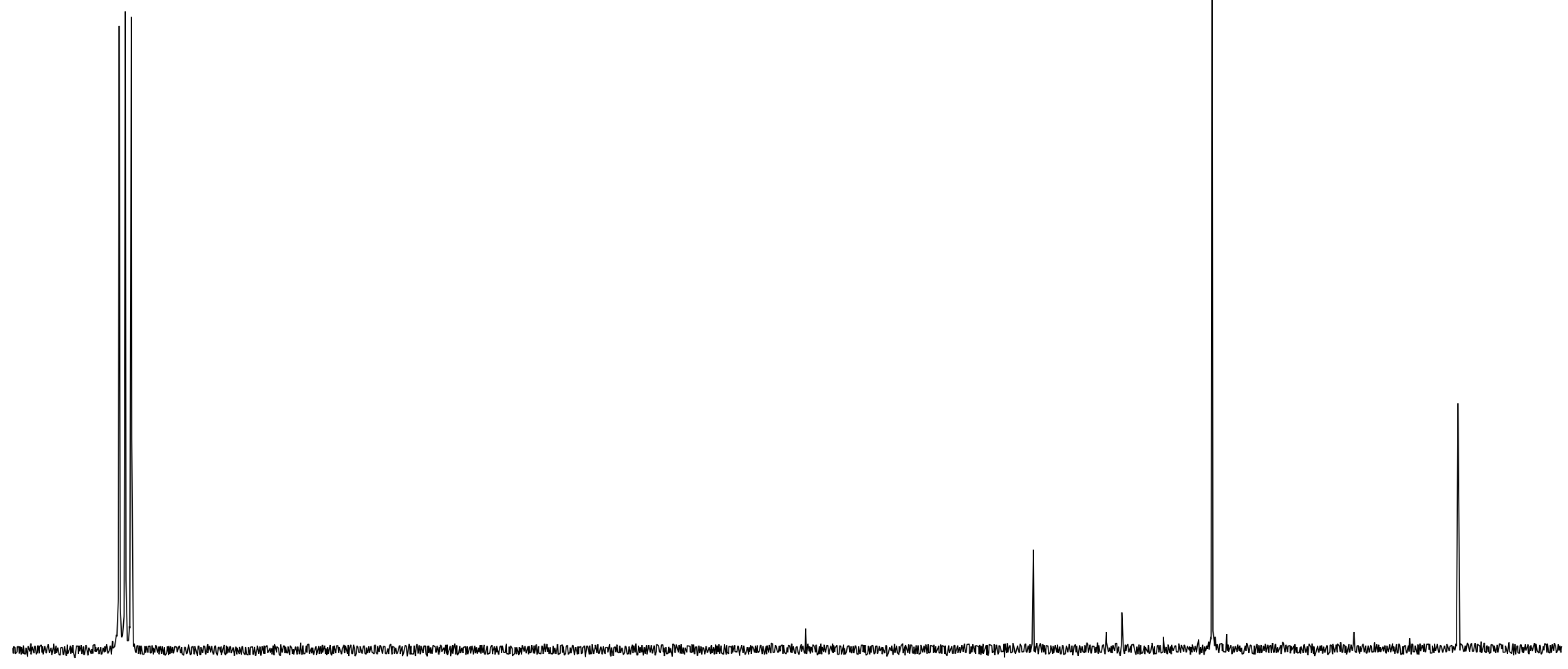

\begin{tabular}{llllllllll|l|l|l|l}
80 & 75 & 70 & 65 & 60 & 55 & $\begin{array}{c}50 \\
\mathrm{f}(\mathrm{ppm})\end{array}$ & 45 & 40 & 35 & 30 & 25
\end{tabular}


${ }^{1} \mathrm{H}$ NMR $\left(500 \mathrm{MHz}, \mathrm{CDCl}_{3}\right)$

OMe

39

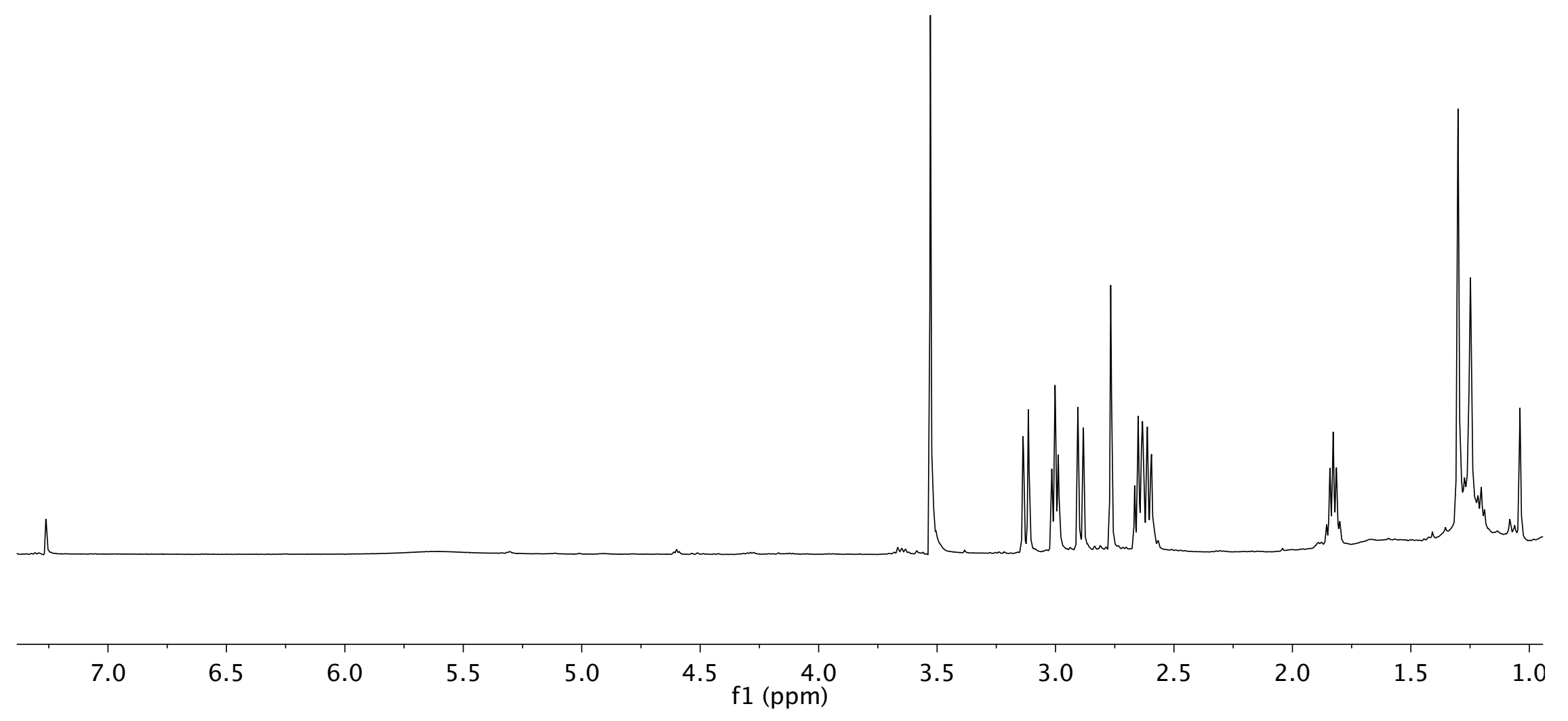


${ }^{13} \mathrm{C}$ NMR $\left(125 \mathrm{MHz}, \mathrm{CDCl}_{3}\right)$

$\underbrace{\mathrm{OMe}}_{39}$

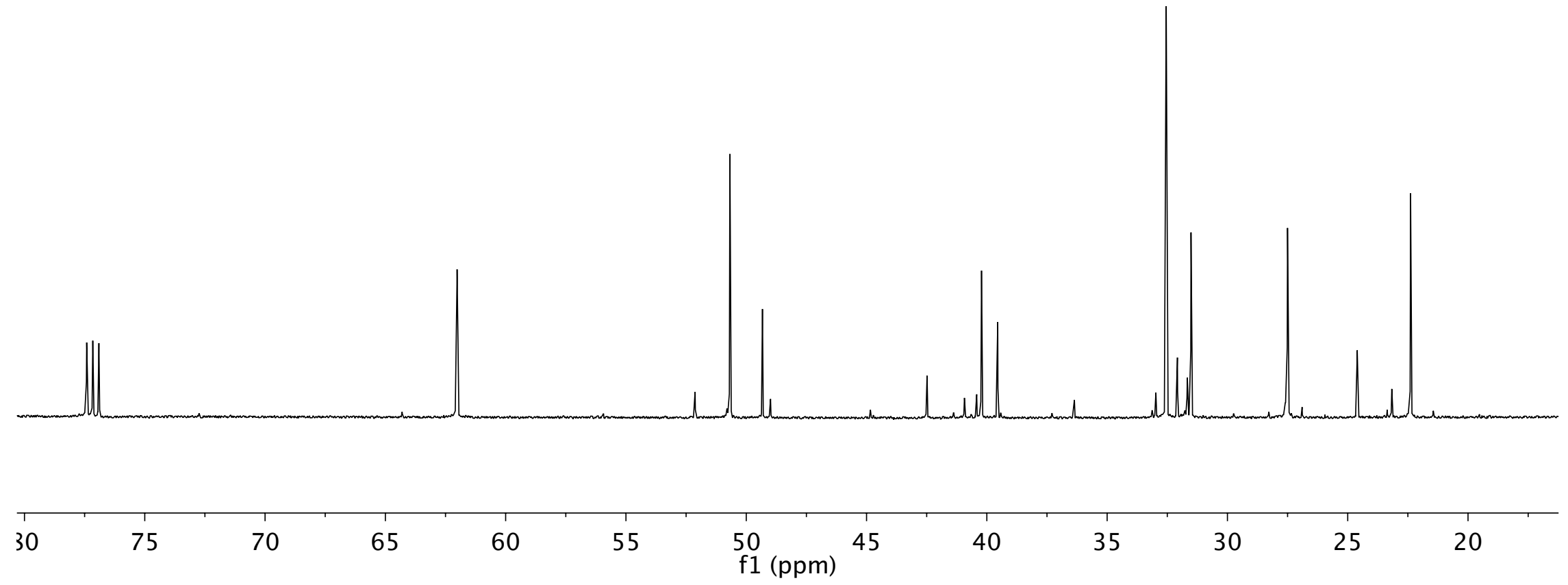


${ }^{1} \mathrm{H}$ NMR (300 MHz, $\left.\mathrm{CDCl}_{3}\right)$

$\overbrace{41}^{\mathrm{Br}} \mathrm{Br}$
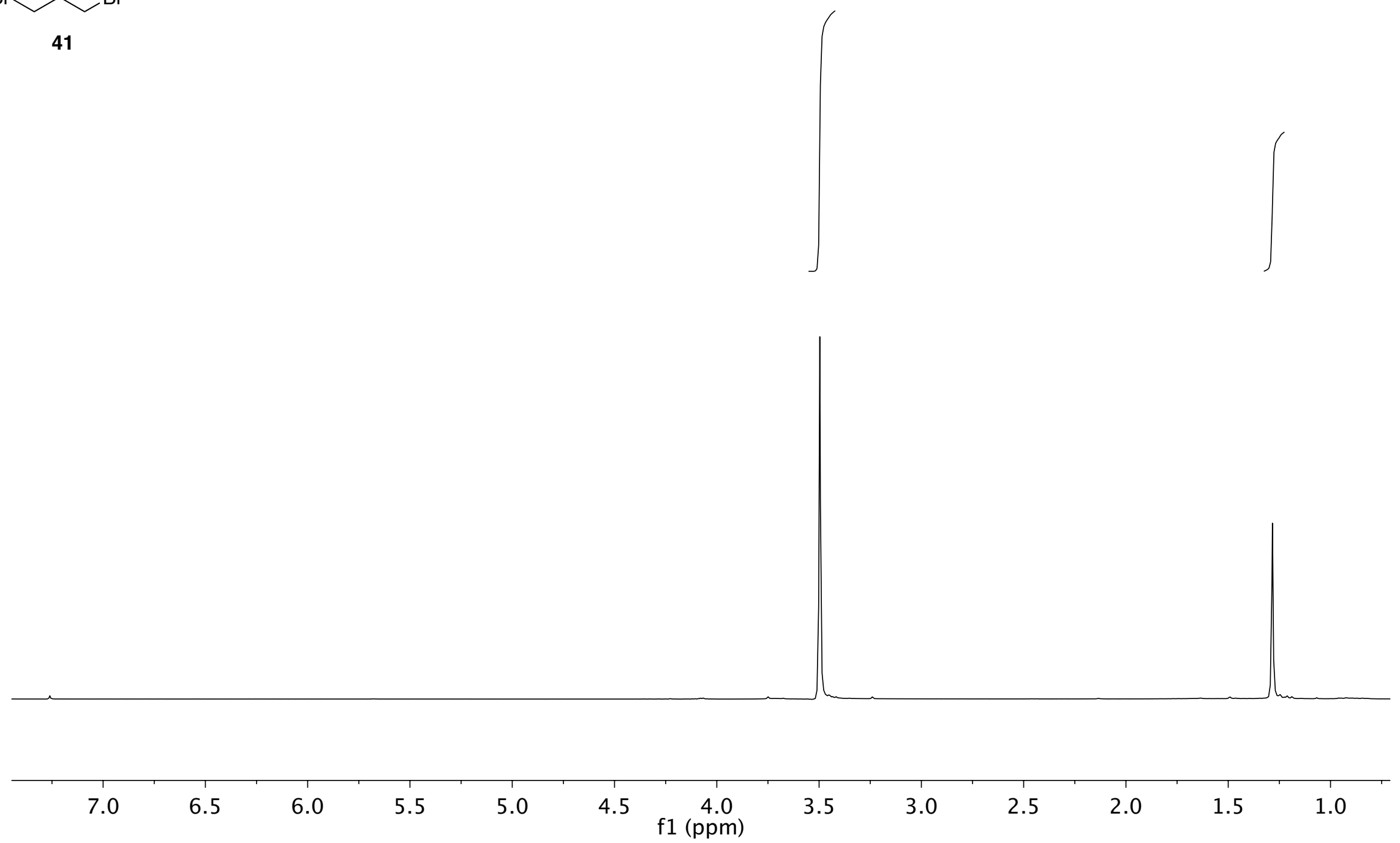
${ }^{13} \mathrm{C} \mathrm{NMR}\left(125 \mathrm{MHz}, \mathrm{CDCl}_{3}\right)$

$\int_{41}^{\mathrm{Br}} \mathrm{Br}$
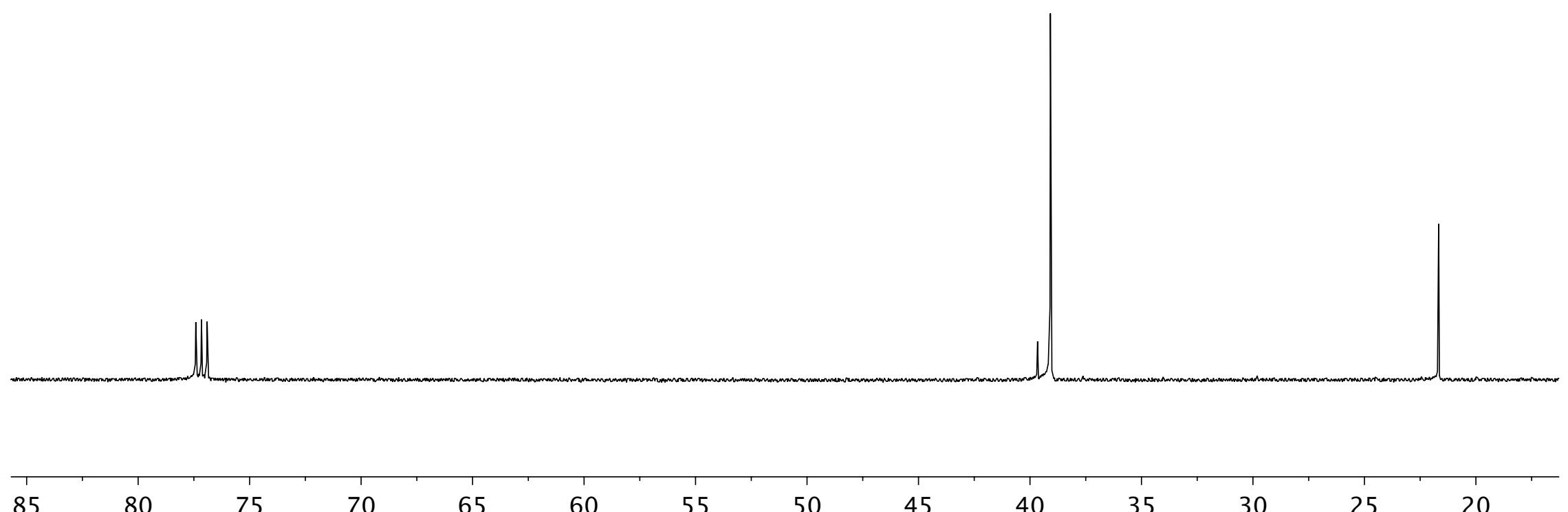

65

60

55

$\mathrm{f} 1(\mathrm{ppm})$

45

40

35

30

25

20 
${ }^{1} \mathrm{H} \mathrm{NMR}\left(500 \mathrm{MHz}, \mathrm{CDCl}_{3}\right)$

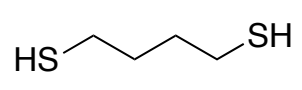

43

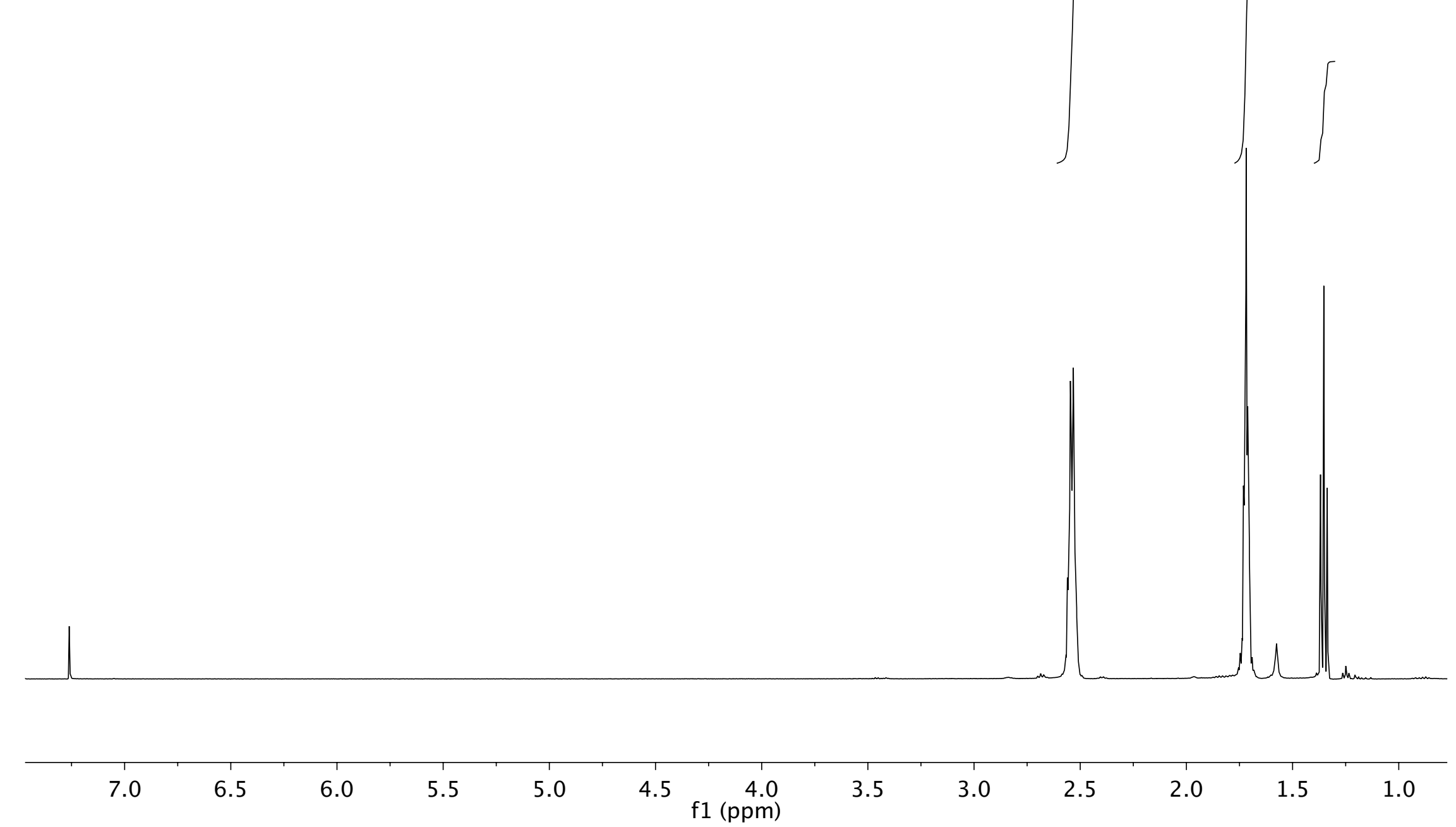


${ }^{13} \mathrm{C}$ NMR (125 MHz, $\left.\mathrm{CDCl}_{3}\right)$

$\mathrm{HS}_{\mathrm{SH}}^{\sim}$

43

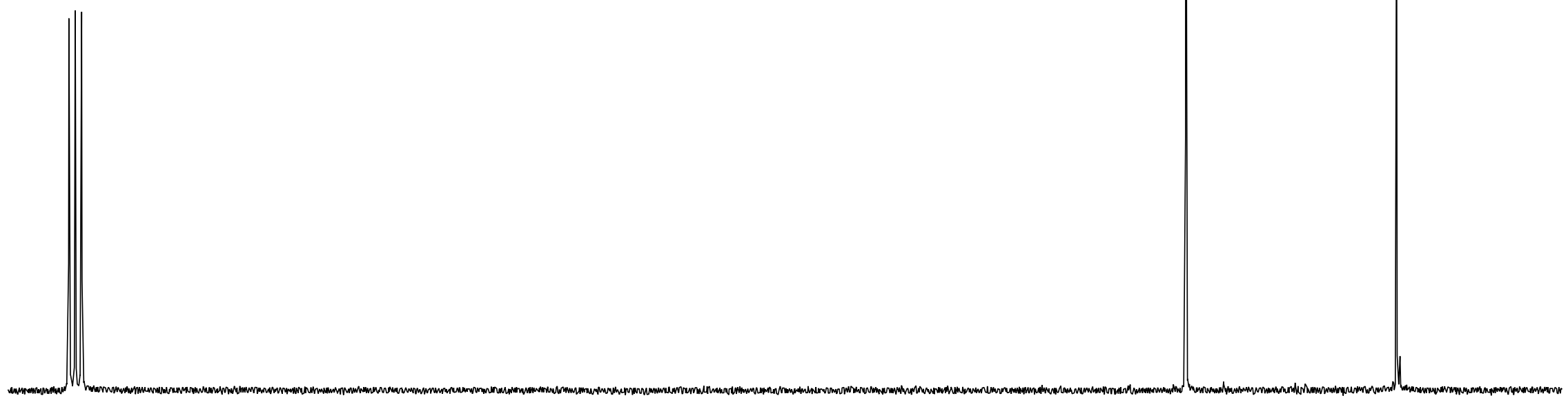

75

70

65

60

55

50

45

40

35

30

25

20 
${ }^{1} \mathrm{H} \mathrm{NMR}\left(500 \mathrm{MHz}, \mathrm{CDCl}_{3}\right)$

$\overbrace{\mathrm{Br}}^{\mathrm{Br}} \mathrm{Br}$

45
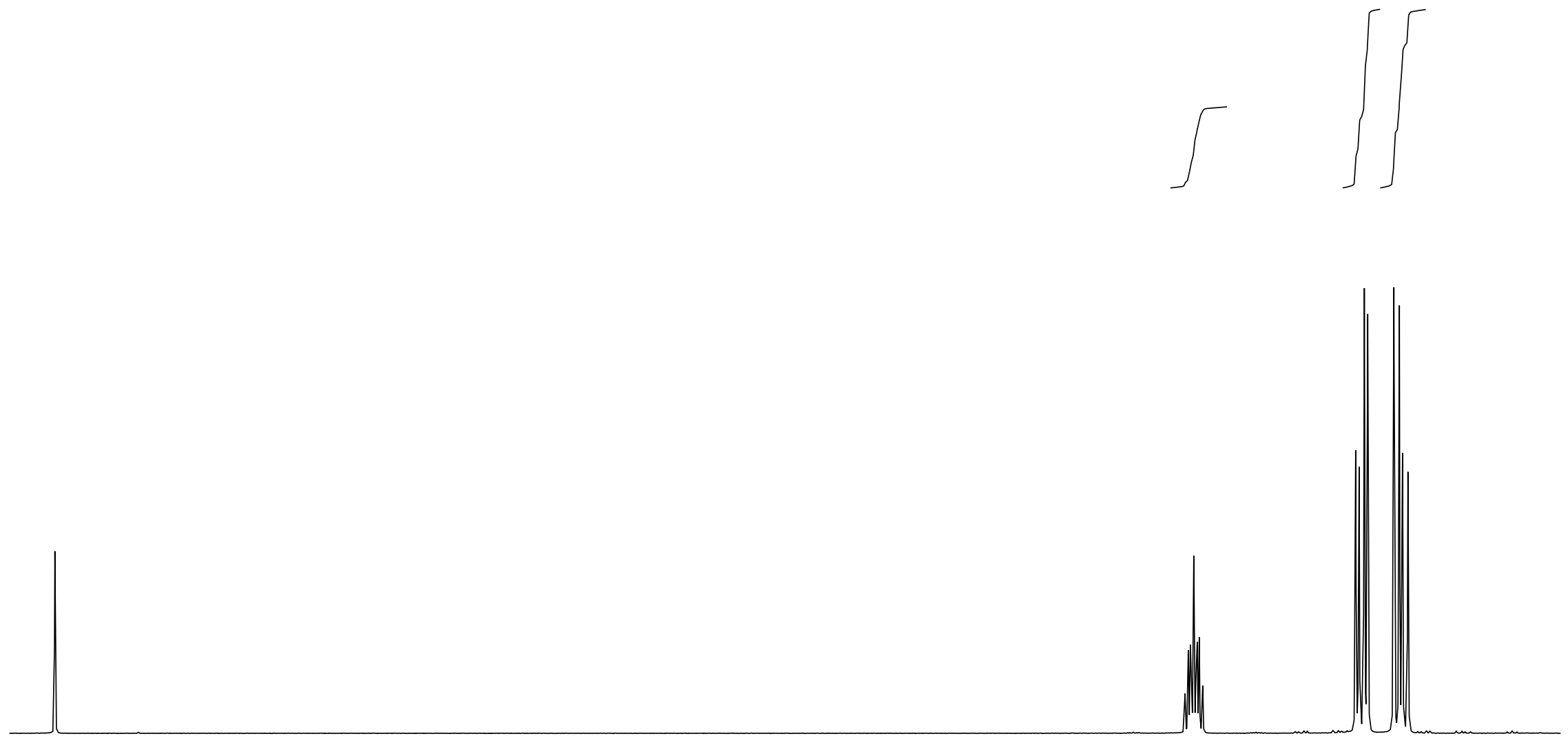

$\begin{array}{llllllllllllllllllll}7.3 & 7.1 & 6.9 & 6.7 & 6.5 & 6.3 & 6.1 & 5.9 & 5.7 & 5.5 & 5.3 & 5.1 & 4.9 & 4.7 & 4.5 & 4.3 & 4.1 & 3.9 & 3.7 & 3.5\end{array}$ 
${ }^{13} \mathrm{C} \operatorname{NMR}\left(125 \mathrm{MHz}, \mathrm{CDCl}_{3}\right)$

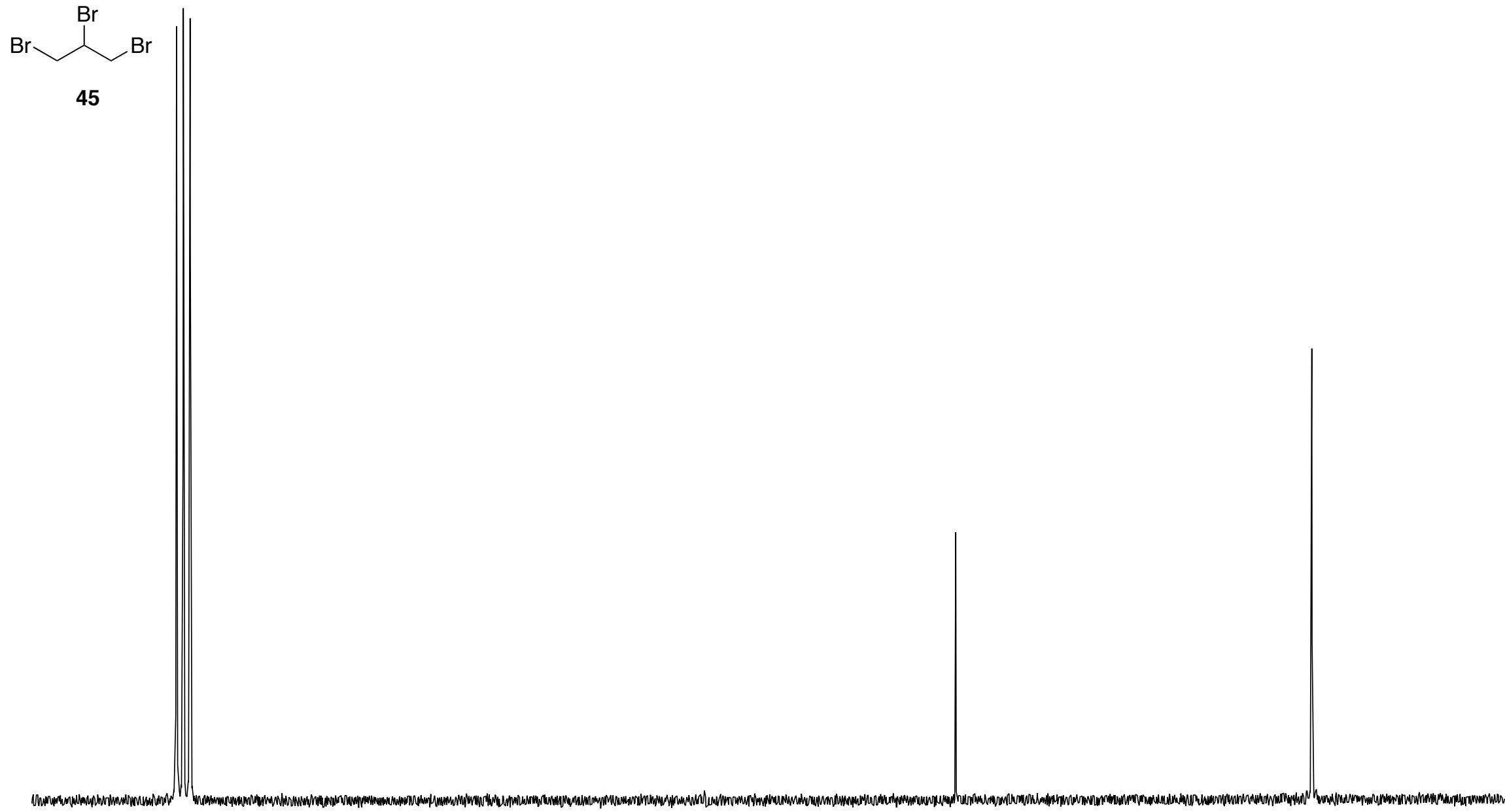

\begin{tabular}{lllllllll}
\hline 80 & 75 & 70 & 65 & 60 & $\begin{array}{c}55 \\
\mathrm{f} 1(\mathrm{ppm})\end{array}$ & 50 & 45 & 40
\end{tabular}



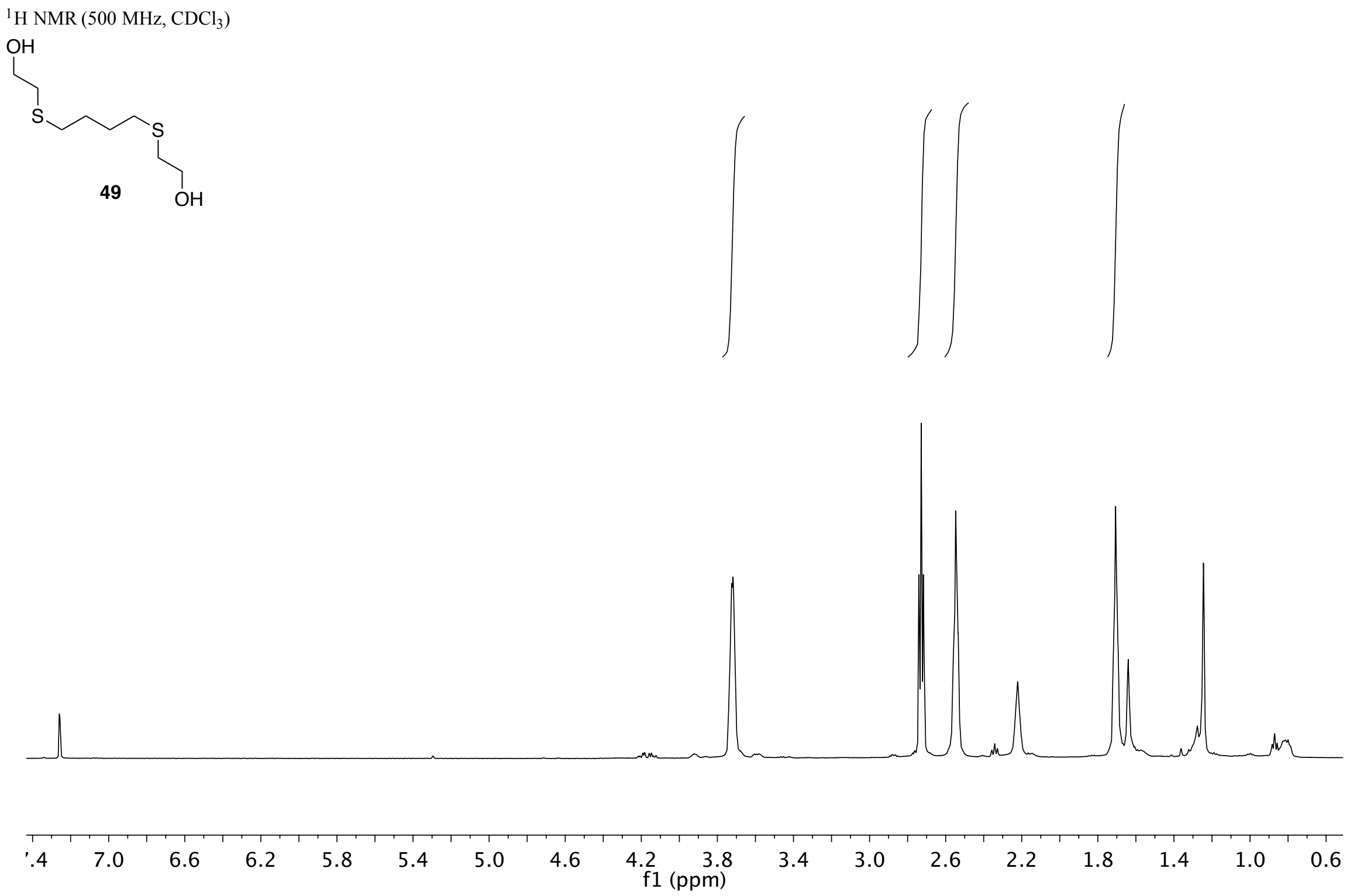


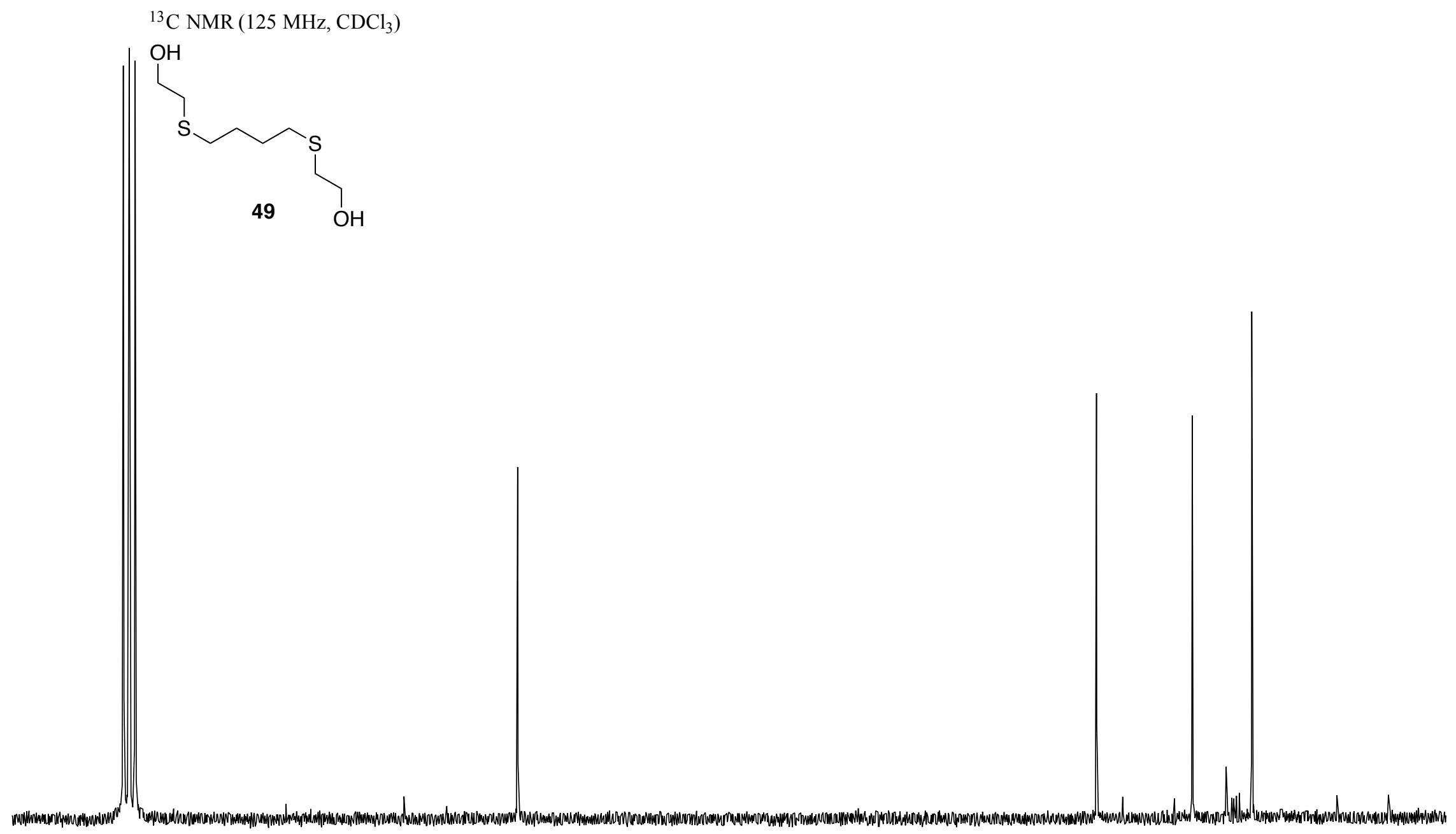

$\begin{array}{llllllllllllllllllllllllllllllll}32 & 80 & 78 & 76 & 74 & 72 & 70 & 68 & 66 & 64 & 62 & 60 & 58 & 56 & 54 & 52 & 50 & 48 & 46 & 44 & 42 & 40 & 38 & 36 & 34 & 32 & 30 & 28 & 26 & 24 & 22\end{array}$ 
${ }^{1} \mathrm{H}$ NMR $\left(500 \mathrm{MHz}, \mathrm{CDCl}_{3}\right)$

$\mathrm{SH}$
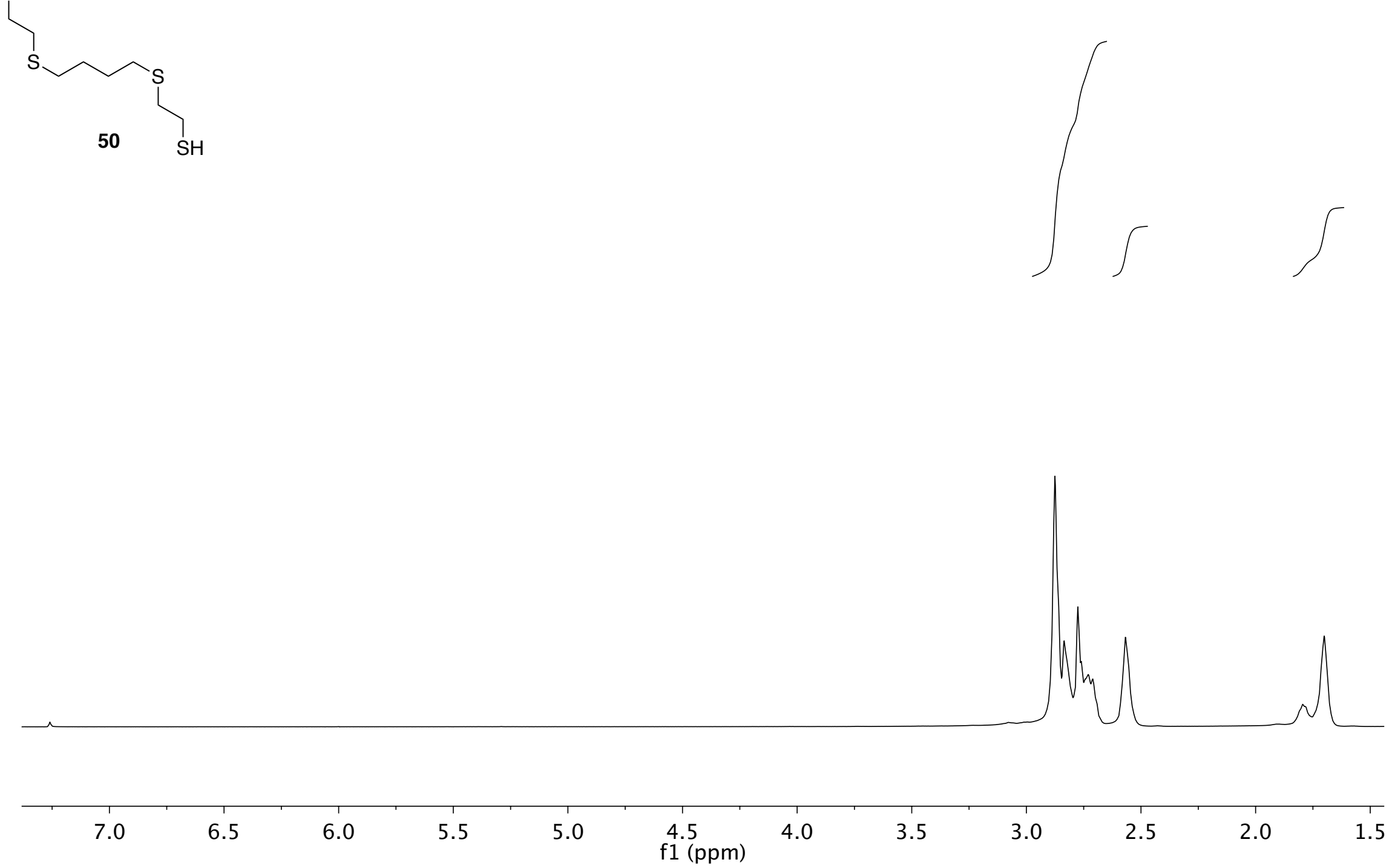


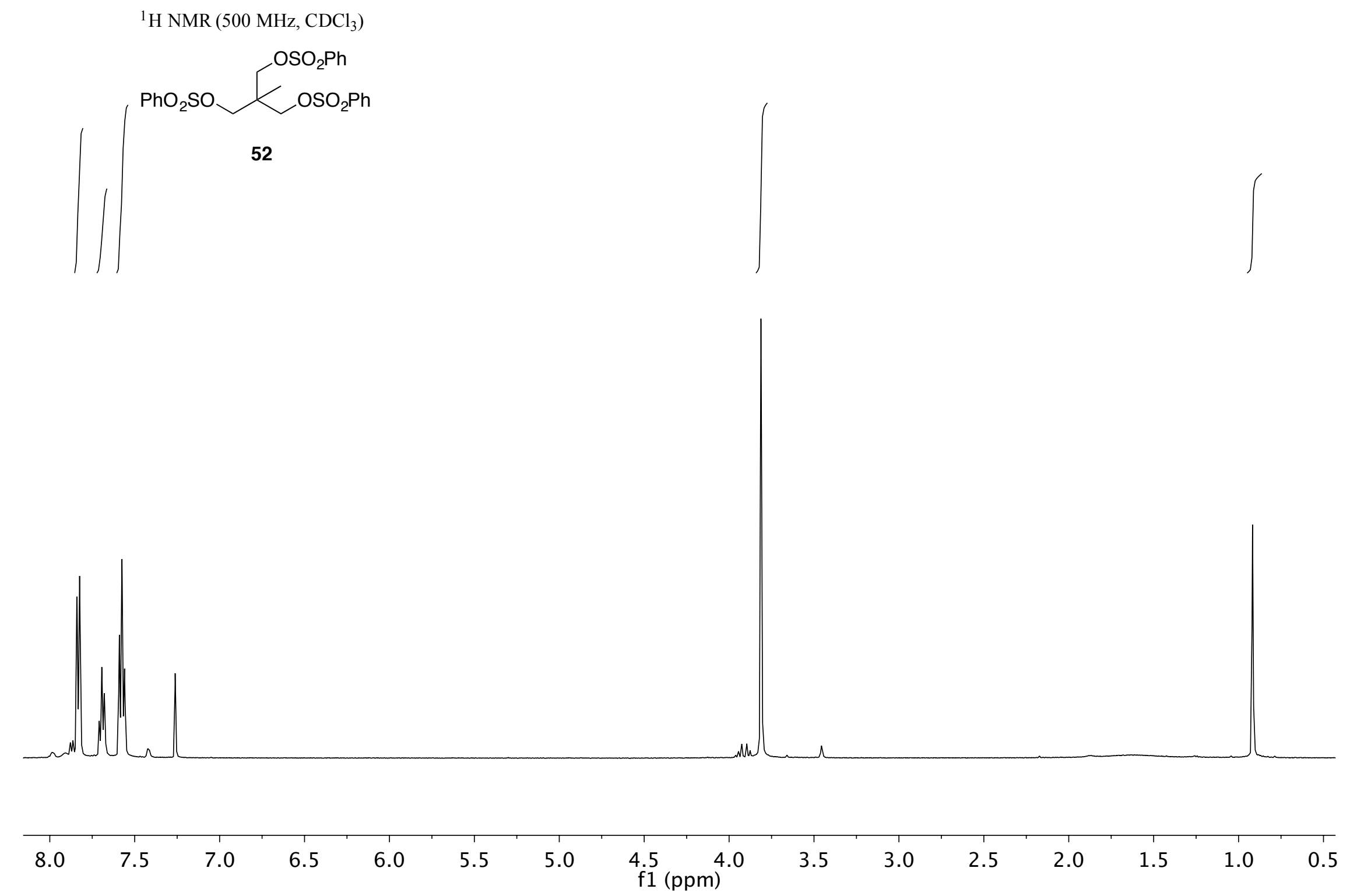


${ }^{13} \mathrm{C}$ NMR (125 MHz, $\mathrm{CDCl}_{3}$ )

$\mathrm{PhO}_{2} \mathrm{SO} \longrightarrow \mathrm{OSO}_{2} \mathrm{Ph}$

52

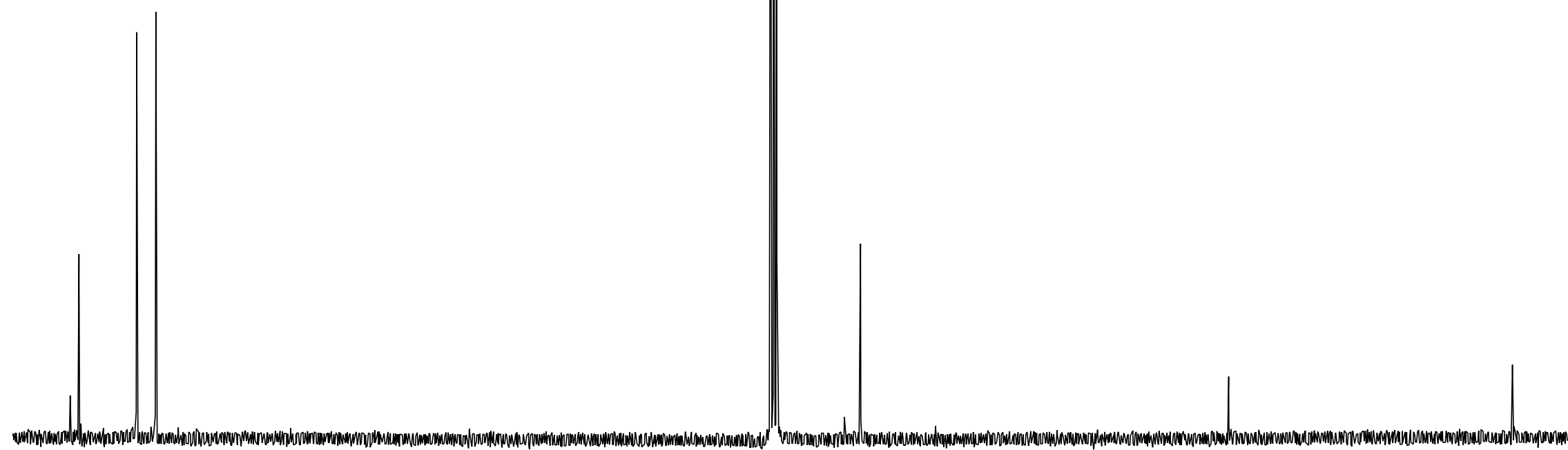


${ }^{1} \mathrm{H}$ NMR (500 MHz, D 20 )

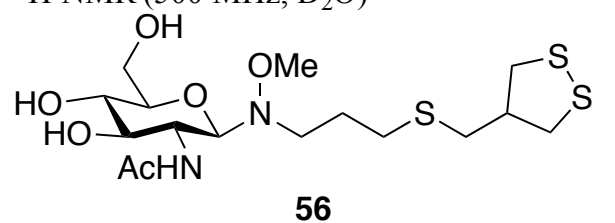

56
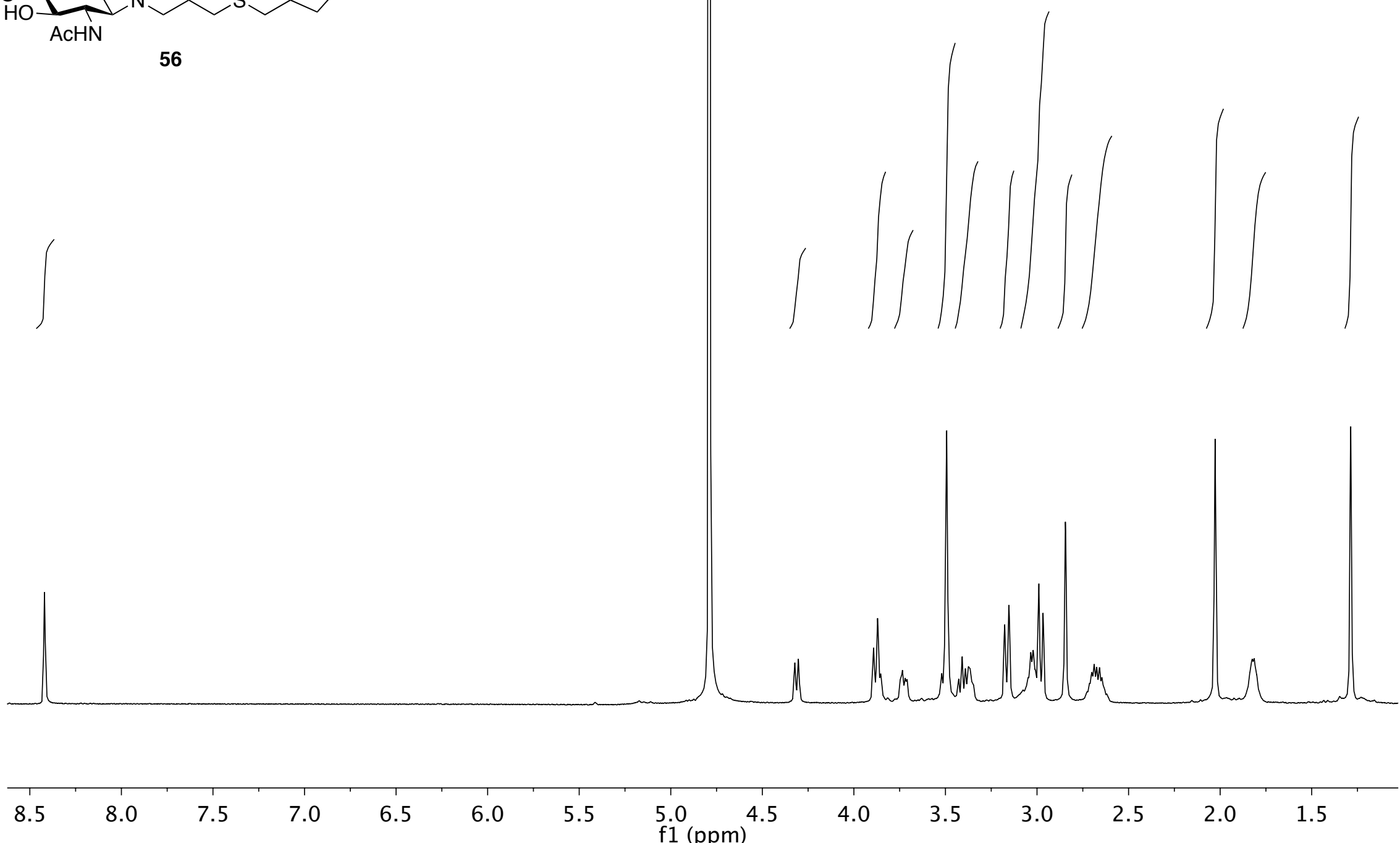
${ }^{13} \mathrm{C}$ NMR $\left(125 \mathrm{MHz}, \mathrm{D}_{2} \mathrm{O}\right)$

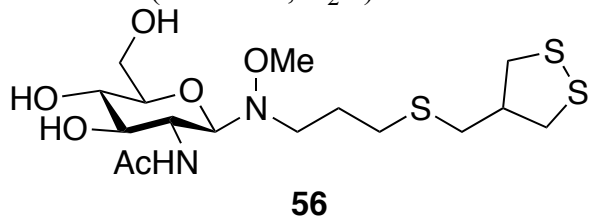

56

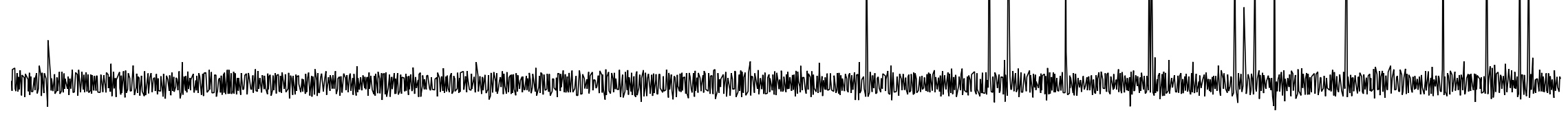

\begin{tabular}{|c|c|c|c|c|c|c|c|c|c|c|c|c|c|c|c|}
\hline 30 & 170 & 160 & 150 & 140 & 130 & 120 & 110 & $\begin{array}{c}100 \\
1(\mathrm{ppm})\end{array}$ & 90 & 80 & 70 & 60 & 50 & 40 & 30 \\
\hline
\end{tabular}

